\title{
The most-continuous part of the Plancherel decomposition for a reductive symmetric space
}

\author{
E.P. van den Ban and H. Schlichtkrull
}

August 9, 1995

\section{Contents}

1 Introduction $\quad 2$

$\begin{array}{lll}2 & \text { Notation and preliminaries } & 7\end{array}$

3 Normalization of measures $r$

4 The Fourier transform $\quad 12$

5 Eisenstein integrals $\quad 14$

6 The $\tau$-spherical Fourier transform $\quad 20$

7 Expansions of the Eisenstein integral $\quad 22$

8 Paley-Wiener estimates for the Fourier transform 26

$9 \quad$ Wave packets $\quad 33$

10 Support properties of wave packets $\quad 36$

11 The behavior of supports 41

12 Asymptotic behavior of differential operators 43

13 A limit behavior of the spherical Fourier transform 53

14 Fourier inversion modulo a differential operator $\quad 57$

15 Injectivity of the Fourier transforms $\quad 59$ 


\section{Introduction}

Let $G / H$ be a semisimple symmetric space, that is, $G$ is a connected semisimple real Lie group with an involution $\sigma$, and $H$ is an open subgroup of the group of fixed points for $\sigma$ in $G$. The fundamental problem in harmonic analysis on $G / H$ is to obtain an explicit direct integral ('Plancherel') decomposition

$$
L \simeq \int_{\hat{G}}^{\oplus} m_{\pi} \pi d \mu(\pi)
$$

of the regular representation $L$ of $G$ in $L^{2}(G / H)$ into irreducible unitary representations. The principal result of this paper is the determination of such a decomposition for the part of $L^{2}(G / H)$ which corresponds to the 'most-continuous part' of the spectrum.

In order to explain what is meant by the 'most-continuous part', let us consider for a moment the important particular case of the group $G$ itself considered as a symmetric space for the left times right action of $G \times G$ (the 'group case'). As is well known an explicit decomposition of the form (1) has then been determined by Harish-Chandra ([25]-[27]). The components of $L^{2}(G)$ fall in a (finite) number of 'series', each of which corresponds to a particular (class of) cuspidal parabolic subgroup $P$ and is a direct integral of representations $\pi_{P, \xi, \lambda} \otimes \pi_{P, \xi, \lambda}^{*}$. Here $\pi_{P, \xi, \lambda}$ belongs to the principal series induced from $P=M A N, \xi$ is in the discrete series of $M$, and $\lambda$ is a continuous parameter ranging in the imaginary linear dual $i \mathfrak{a}^{*}$ of $\mathfrak{a}$ (the Lie algebra of $A$ ). By definition, the most-continuous part of $L^{2}(G)$ is the component part associated with a $P$ with maximal $\mathfrak{a}$-part, that is, a minimal parabolic subgroup.

Returning to the general case of $G / H$, let $\mathfrak{g}$ be the Lie algebra of $G$, and let $\mathfrak{g}=$ $\mathfrak{h} \oplus \mathfrak{q}$ be its decomposition into \pm 1-eigenspaces for $\sigma$ (so that $\mathfrak{h}$ is the Lie algebra of $H$ ). Furthermore, let $\theta$ be a Cartan involution commuting with $\sigma$. By analogy with the group case one expects $L^{2}(G / H)$ to decompose into a finite number of parts, each attached to a particular parabolic subgroup $P$ satisfying $\sigma \theta(P)=P$. The part attached to $P=M A N$ has the form of a direct integral of generalized principal series representations $\pi_{\xi, \lambda}=\pi_{P, \xi, \lambda}$, with data essentially as follows. The parabolic subgroup $P=M A N$ has $\sigma$-stable $M$ and $A$ parts, $\xi$ is in the discrete series for the symmetric space $M /(M \cap H)$, and $\lambda \in i \mathfrak{a}^{*}$ 
vanishes on $\mathfrak{a} \cap \mathfrak{h}$. The 'most-continuous part' would then be the part corresponding to the parabolic subgroup $P=M A N$ as above for which $\mathfrak{a} \cap \mathfrak{q}$ is maximal, which is the socalled $\sigma$-minimal parabolic subgroup. For this parabolic subgroup the space $M /(M \cap H)$ is compact, and hence only finite dimensional $\xi$ 's occur. In this paper we determine explicitly such a direct integral

$$
\int_{\xi, \lambda}^{\oplus} m_{\xi} \pi_{\xi, \lambda} d \mu(\xi, \lambda)
$$

for this parabolic subgroup, together with an equivariant imbedding into $L^{2}(G / H)$ (the multiplicity $m_{\pi}=m_{\xi}$ of $\pi_{\xi, \lambda}$, explicitly given below, happens to be independent of $\lambda$ ). The most-continuous part of $L^{2}(G / H)$, denoted by $L_{\mathrm{mc}}^{2}(G / H)$, is then by definition the image of (2). We realize the inverse map from $L_{\mathrm{mc}}^{2}(G / H)$ to $(2)$ explicitly by means of the Fourier transform $f \mapsto \hat{f}$ defined in [13]. We also prove that the orthocomplement $L_{0}^{2}(G / H)$ of $L_{\mathrm{mc}}^{2}(G / H)$ is 'small' in a certain spectral sense (see below). In particular, if $G / H$ has split rank one, that is if the maximal abelian subspace $\mathfrak{a} \cap \mathfrak{q}$ of $\mathfrak{p} \cap \mathfrak{q}$ is one-dimensional, then $L_{0}^{2}(G / H)$ decomposes discretely.

The discrete series for $L^{2}(G / H)$ (which is obtained in the above scheme with $P=G$ ), has been extensively studied, and is by now quite well understood (see [20], [35]). In particular, in the above-mentioned case of split rank one, where the discrete series is complementary to $L_{\mathrm{mc}}^{2}(G / H)$, one can now determine the full decomposition of $L^{2}(G / H)$ by combination of these results with the present work. Previously such an explicit decomposition was known for spaces of rank one (which means that $\mathfrak{a} \cap \mathfrak{q}$ in addition to being one dimensional is maximal abelian in $\mathfrak{q}$ ), see for example [19], [18], [34]. For the symmetric spaces $\mathrm{GL}(n, \mathbb{C}) / \mathrm{U}(p, q)$ a Plancherel decomposition has been obtained in [15]; note that these symmetric spaces are of type $G_{\mathbb{C}} / G_{\mathbb{R}}$; a complex reductive group modulo a real form.

Besides the group case, another important particular case is when $G / H$ is a Riemannian symmetric space of the non-compact type, in which case $H$ is compact. In this case there is a well-developed theory of harmonic analysis, primarily due to Harish-Chandra and Helgason ([22], [23], [24] and [29]). We shall see that $L_{\mathrm{mc}}^{2}(G / H)=L^{2}(G / H)$ in this case. Thus we retrieve in (2) the Plancherel decomposition of $L^{2}(G / H)$. A major simplification of the proof of this decomposition was found by Rosenberg [36], who used techniques inspired by Helgason's Paley-Wiener theorem (see also [30], IV, §7).

For the present generalization to arbitrary $G / H$ we use ideas inspired by those of Rosenberg. In fact, we also obtain a Paley-Wiener theorem for $G / H$ (that is, we determine the Fourier image of the space $C_{c}^{\infty}(G / H)_{K}$ of $K$-finite compactly supported smooth functions), but only in the case where $G / H$ has split rank one. For the group case a similar theorem was obtained in [16] for groups of split rank one, and in [2] for groups of arbitrary split rank. For spaces $G / H$ of general rank we prove the injectivity of our Fourier transform on compactly supported functions and pose a conjectural image space.

We shall now describe our results in more detail. Let $P=M A N$ be a $\sigma$-minimal parabolic subgroup as above, let $\xi$ be a finite dimensional unitary representation of $M$, and let $\lambda \in \mathfrak{a}_{\mathbf{q} \mathfrak{c}}^{*}$, where $\mathfrak{a}_{\mathfrak{q}}=\mathfrak{a} \cap \mathfrak{q}$. Furthermore, let $C^{\infty}(\xi: \lambda)$ and $C^{-\infty}(\xi: \lambda)$ denote 
the spaces of smooth, respectively generalized, vectors for $\pi_{\xi, \lambda}$, and let $C^{-\infty}(\xi: \lambda)^{H}$ be the space of $H$-fixed elements in $C^{-\infty}(\xi: \lambda)$. In [6], [13] a certain finite dimensional space $V(\xi)$ (independent of $\lambda$ ) was defined together with a natural family of linear maps $j^{\circ}(\xi: \lambda)=j^{\circ}(P: \xi: \lambda): V(\xi) \rightarrow C^{-\infty}(\xi: \lambda)^{H}$, depending meromorphically on $\lambda \in \mathfrak{a}_{\mathrm{q} c}^{*}$ and bijective for generic $\lambda$. The definition involved a normalization procedure which guarantees that $\lambda \mapsto j^{\circ}(\xi: \lambda)$ is regular at the imaginary points $\lambda \in i \mathfrak{a}_{\mathrm{q}}^{*}$ (cf. [13]). This is crucial for the definition of the Fourier transform associated with (2). For $f \in C_{c}^{\infty}(G / H)$ one defines

$$
\begin{aligned}
\hat{f}(\xi: \lambda) & =\int_{G / H} f(g H) \pi_{\xi,-\lambda}(g) j^{\circ}(\xi:-\lambda) d(g H) \\
& \in \operatorname{Hom}\left(V(\xi), C^{\infty}(\xi:-\lambda)\right) \simeq V(\xi)^{*} \otimes C^{\infty}(\xi:-\lambda)
\end{aligned}
$$

for $\lambda \in i \mathfrak{a}_{\mathrm{q}}^{*}$.

Our main result, stated in Theorems 18.8 and 18.9, now asserts that if one sets $d \mu(\xi, \lambda)=\operatorname{dim}(\xi) d \lambda$, where $d \lambda$ is Lebesgue measure (suitably normalized) on $i \mathfrak{a}_{\mathrm{q}}^{*}$, then $f \mapsto \hat{f}$ extends to a partial isometry of $L^{2}(G / H)$ onto the space given by (2), with specified domains for $\xi$ and $\lambda$.

In the group case as well as in the Riemannian case the Plancherel theorem is proved by reduction to $K$-finite functions on $G / H$, and so is our theorem here (in the Riemannian case one may even reduce to $K$-invariant functions on $G / K$, but for general $G / H$ other $K$-types than the trivial one are needed). For functions on $G / H$ transforming according to a given $K$-type $\tau$, a Fourier transform closely related to the one above can be defined with the use of normalized Eisenstein integrals $E^{\circ}(\psi: \lambda)$ on $G / H$. These are generalizations of Harish-Chandra's normalized Eisenstein integrals associated with a minimal parabolic subgroup in the group case. In the Riemannian case a $K$-invariant normalized Eisenstein integral is essentially equal to $c(\lambda)^{-1} \varphi_{\lambda}$, an elementary spherical function divided by the associated $c$-function. The Eisenstein integrals as well as their normalized versions were defined and studied in the paper [7], on which the present paper heavily relies. They are linear combinations of matrix coefficients of $K$-finite vectors of type $\tau$ with the $H$-fixed vectors $j^{\circ}(\xi: \lambda) \eta, \eta \in V(\xi)$, and they depend meromorphically on the parameter $\lambda \in \mathfrak{a}_{\mathrm{q} c}^{*}$ and linearly on the parameter $\psi \in{ }^{\circ} \mathcal{C}(\tau)$. Here ${ }^{\circ} \mathcal{C}(\tau)$ is a certain finite dimensional space depending on $\tau$ (see Section 5 for its definition). The Eisenstein integrals behave finitely under the algebra $\mathbb{D}(G / H)$ of invariant differential operators on $G / H$, and they can be represented by converging series expansions describing their asymptotic behavior at infinity. From the leading terms of these expansions normalized $c$-functions related to $G / H$ were obtained in $[7]$.

In the present paper we need the normalized Eisenstein integral rather than the unnormalized one, because of the crucial fact (established in [13]) that $\lambda \mapsto E^{\circ}(\psi: \lambda)$ is regular at points $\lambda \in i \mathfrak{a}_{\mathrm{q}}^{*}$. This is closely related to the regularity of the $j^{\circ}(\xi: \lambda)$ at imaginary values of $\lambda$.

In Section 7 we give a converging expansion for the Eisenstein integral and a uniform estimate for its coefficients. In the Riemannian case this specializes to the estimate of [21] which is crucial for Helgason's Paley-Wiener theorem as well as for Rosenberg's proof of 
the Plancherel theorem. Likewise the estimate plays a crucial role in the present paper. It will be proved in [14].

The Fourier transform $\mathcal{F} f(\lambda)$ of a (Schwartz-) function $f$ on $G / H$ transforming according to the $K$-type $\tau$ is defined by integration of $f$ against $E^{\circ}(\psi: \lambda)$; it is a ${ }^{\circ} \mathcal{C}(\tau)$-valued Schwartz function on $i \mathfrak{a}_{\mathrm{q}}^{*}$. If $f$ is compactly supported, then $\mathcal{F} f$ has a meromorphic extension to $\mathfrak{a}_{\mathrm{q} c}^{*}$; in Section 8 we derive an estimate of Paley-Wiener type for $\mathcal{F} f$. As in the classical case the precise estimate is controlled by the size of the support of $f$. In comparison with the Riemannian case it is a serious complication that the Eisenstein integrals are obtained only by analytic continuation in $\lambda$ from a region in $\mathfrak{a}_{\mathrm{q} c}^{*}$ which is smaller than the region one wants to cover (for instance, $i \mathfrak{a}_{q}^{*}$ is in general not contained in the initial region). This problem was overcome in [7], and we base our Paley-Wiener estimate on an initial estimate from there.

In Sections 9 and 10 we study 'wave packets'. These are superpositions of normalized Eisenstein integrals: The wave packet transform $\mathcal{J} \varphi$ of a ${ }^{\circ} \mathcal{C}(\tau)$-valued function $\varphi$ on $i \mathfrak{a}_{\mathrm{q}}^{*}$ (with suitable decay) is given by integration of $\varphi$ against $E^{\circ}$; thus $\mathcal{J} \varphi$ is a function on $G / H$ of type $\tau$. It is easily seen that the transforms $\mathcal{F}$ and $\mathcal{J}$ are the transposed of each other (that is, with $L^{2}$-type inner products on the respective function spaces one has $\langle\mathcal{F} f \mid \varphi\rangle=\langle f \mid \mathcal{J} \varphi\rangle)$. It is the wave packet transform that furnishes the imbedding of (2) into $L^{2}(G / H)$, for functions of type $\tau$; thus the part of $L_{\mathrm{mc}}^{2}(G / H)$ that transforms according to $\tau$ is the closure in $L^{2}(G / H)$ of the space of all wave packets $\mathcal{J} \varphi$ with a function $\varphi$ of Schwartz decay. In the Riemannian case the inversion formula for the spherical Fourier transform essentially asserts that $\mathcal{J}$ is the inverse of $\mathcal{F}$ (in the formulation of Harish-Chandra the Plancherel measure involves a factor $|\mathbf{c}(\lambda)|^{-2}$, but in our description the factor is incorporated in the normalizations). In the general case, where other series than the most-continuous are present, one can only expect $\mathcal{J}$ to be a partial inverse of $\mathcal{F}$. A key step in Rosenberg's proof for $G / K$ is the use of Helgason's 'shift argument', where the integration in the wave packet is moved away from $i \mathfrak{a}_{\mathrm{q}}^{*}$ in the direction of the negative Weyl chamber. This allows one to detect the size of the support of $\mathcal{J} \varphi$ from a Paley-Wiener estimate of $\varphi$ (see [30], IV, Thm. 7.3). Carrying out a similar shift for $G / H$ one would have to take residues into account, because the normalized Eisenstein integrals are in general not holomorphic; the presence of these residues would then be responsible for the other series in $L^{2}(G / H)$. A priori it is however not clear that these residues correspond to the other series. Instead of tackling the problem directly, we circumvent it in the following way. The singularities met in the shift argument are located on a finite number of hyperplanes, and it is possible to choose a (non-trivial) invariant differential operator $D$ so that all the singularities are cancelled by its presence in the application of the shift to $D \mathcal{J} \varphi$. The outcome, given in Theorem 10.2, is indeed that the size of the support of $D \mathcal{J} \varphi$ is governed by a Paley-Wiener estimate of $\varphi$. Notice that the differential operator $D$ depends on $\tau$.

Since $\mathcal{F} f$ has already been shown to satisfy a Paley-Wiener estimate when $f$ is compactly supported, it follows that the size of the support of $D \mathcal{J} \mathcal{F} f$ is governed by the size of the support of $f$. In Section 11 we refine this result and obtain in Theorem 11.1 that $\operatorname{supp} D \mathcal{J F} f \subset \operatorname{supp} f$ for all compactly supported smooth functions $f$ of type $\tau$. 
The goal of the following Sections 12, 13, 14 is to establish the 'inversion formula', Theorem 14.1, which states that

$$
D \mathcal{J} \mathcal{F}=D
$$

on compactly supported smooth functions of type $\tau$. This formula is a cornerstone in the proof of the main theorem. At this point the compactness of $H$ is crucially used in Rosenberg's argument for the Riemannian case, and hence in our generality we have to introduce a different method. We base the proof of (4) on a theory of 'asymptotic behavior of differential operators' developed in Section 12. It follows from the above mentioned support preserving property of $D \mathcal{J} \mathcal{F}$ that this operator acts as a differential operator on functions of type $\tau$. Moreover, from easy properties of $\mathcal{J}$ and $\mathcal{F}$ the operator $D \mathcal{J} \mathcal{F}$ is seen to belong to the commutant of $\mathbb{D}(G / H)$. We prove that the coefficients of any differential operator that commutes with $\mathbb{D}(G / H)$ on functions of type $\tau$ have converging series expansions similar to that of $E^{\circ}(\psi: \lambda)$, and that the leading terms in the expansions determine the operator uniquely. The formula (4) then follows from a comparison of the asymptotic behavior of $D \mathcal{J F}$ with that of $D$. The crucial part of the comparison is carried out in Section 13.

In Section 15 we establish injectivity of the Fourier transforms $f \mapsto \mathcal{F} f$ and $f \mapsto \hat{f}$, when $f$ ranges over the compactly supported smooth functions (see Theorems 15.1 and 15.5). This is deduced from (4) and a result in [12], which states that the application of an invariant differential operator is injective on $C_{c}^{\infty}(G / H)$, under a certain condition which is met by the $D$ from above.

In Section 16 we invert the order of $\mathcal{F}$ and $\mathcal{J}$ and study the operator $\mathcal{F} \mathcal{J}$ on ${ }^{\circ} \mathcal{C}(\tau)$ valued Schwartz functions on $i \mathfrak{a}_{\mathrm{q}}^{*}$. We prove that this operator is simply given by the projection onto the space of functions satisfying a certain transformation property under a Weyl group. In particular, it follows that if $(\xi, \lambda)$ is restricted to a fundamental domain for the action of this Weyl group, then $\mathcal{J}$ gives rise to an isometric injection of (2) into $L^{2}(G / H)$, for functions of $K$-type $\tau$. At this stage our proof is reminiscent of an argument of Harish-Chandra for the spherical Fourier transform on $G / K$ (see [23], pp. 591-592), but the 'inversion formula' (4) enters crucially at a certain point. On the Fourier transformed side the application of $D$ corresponds to multiplication with a certain polynomial, and since we are dealing with meromorphic functions we are allowed to divide it out.

In Section 18 we take up the study of the Fourier transform $f \mapsto \hat{f}$ for non- $K$-finite functions. Using the results of the previous sections we prove our main theorem, Theorem 18.9, which was outlined above. We also obtain the result mentioned earlier that the orthocomplement $L_{0}^{2}(G / H)$ of $L_{\mathrm{mc}}^{2}(G / H)$ is 'spectrally small': A $K$-finite function $f$ of type $\tau$ in $L_{0}^{2}(G / H)$ is contained in the kernel of the differential operator $D$ (notice however that $D$ depends on $\tau$ ). Another manifestation of the spectral smallness is the result that $L_{0}^{2}(G / H) \cap C_{c}^{\infty}(G / H)=\{0\}$ (Theorem 18.11).

In Section 19 we discuss the dependence of Theorem 18.9 on various choices made along the way.

In Section 20 we establish some results that will be needed in Section 21.

Finally, in Section 21 we derive the Paley-Wiener type result mentioned above for spaces of split rank one. In analogy with the group case (see the references mentioned 
above) the image by $\mathcal{F}$ of the space of compactly supported smooth functions of type $\tau$ is characterized by a growth condition (obtained from the 'Paley-Wiener type' estimate of before), a condition for the transformation under the Weyl group, and an extra set of conditions (not present in the Riemannian case) coming from relations among the Eisenstein integrals. These conditions are set up without the assumption of split rank one in order to give a conjecture for the general case. In comparison with the group case and the Riemannian case the theory is more complicated, because we have to deal with meromorphic functions on $\mathfrak{a}_{\mathrm{qc}}^{*}$ rather than holomorphic functions.

As mentioned above, the present paper owes a great deal to the theory of Helgason and Rosenberg on the spherical Fourier transform for $G / K$. One of the major advantages of Rosenberg's proof of the Plancherel formula, compared with Harish-Chandra's original proof, is that some rather intricate estimates of [23] are avoided. Recently it was shown (see [1]) that also Harish-Chandra's result on Schwartz functions can be derived by the same method. For the present generalization to $G / H$ we have not been able to avoid the use of such Schwartz estimates (see Theorem 16.4); they are given in [9]. Besides the theory for $G / K$ our major source of inspiration has of course been Harish-Chandra's papers [25]-[27] for the group case.

Some of the results of this paper have been announced in [11], where also some further results on the multiplicity function $m_{\pi}$ are discussed and illustrated by examples. In that paper we also give some details on the specialization of the present theory to the group case. Other results of the present paper, as well as applications to multipliers, have been described in the survey paper [10]. Finally we would like to draw attention to the lecture notes [28], where an overview of the theory behind the present paper is given.

\section{Notation and preliminaries}

Let $G$ be a real reductive Lie group of Harish-Chandra's class (cf. [25]), $\sigma$ an involution of $G$, and $H$ an open subgroup of the group $G^{\sigma}$ of its fixed points. Then $G / H$ is a reductive symmetric space. Let $\theta$ be a Cartan involution commuting with $\sigma$, with corresponding maximal compact subgroup $K$, and let $\mathfrak{g}=\mathfrak{h}+\mathfrak{q}$ and $\mathfrak{g}=\mathfrak{k}+\mathfrak{p}$ be the decompositions of the Lie algebra $\mathfrak{g}$ induced by $\sigma$ and $\theta$ (we follow the custom of denoting the Lie algebra of a Lie group by the corresponding lower case German letter). As usual, the Killing form on $[\mathfrak{g}, \mathfrak{g}]$ is extended to an invariant bilinear form $B$ on $\mathfrak{g}$, for which the inner product $\langle X, Y\rangle=-B(X, \theta Y)$ is positive definite. We also require the extension to be compatible with $\sigma$, that is, $B(\sigma X, Y)=B(X, \sigma Y)$ for all $X, Y \in \mathfrak{g}$.

Fix a maximal abelian subspace $\mathfrak{a}_{\mathfrak{q}}$ of $\mathfrak{p} \cap \mathfrak{q}$, and put $A_{\mathfrak{q}}=\exp \mathfrak{a}_{\mathrm{q}}$. Let $M_{1}$ be the centralizer of $\mathfrak{a}_{\mathrm{q}}$ in $G$. We denote by $\Sigma$ the root system of $\mathfrak{a}_{\mathrm{q}}$ in $\mathfrak{g}$. Each positive system $\Sigma^{+}$for $\Sigma$ determines a parabolic subgroup $M_{1} N$, where $N$ is the exponential of the sum $\mathfrak{n}$ of the positive root spaces. In this way there is a one-to-one correspondence between the positive systems for $\Sigma$ and the $\sigma$-minimal parabolic subgroups (that is, those which are minimal with respect to being $\sigma \theta$-stable) containing $A_{\mathrm{q}}$. We write $\mathcal{P}_{\sigma}^{\min }$ for this set of parabolic subgroups, $\Sigma(P)$ for the positive system corresponding to a given 
$P \in \mathcal{P}_{\sigma}^{\min }$, and $\Delta(P)$ for its subset of simple roots. Similarly we write $\mathfrak{a}_{\mathrm{q}}^{+}(P)$ for the corresponding positive open Weyl chamber in $\mathfrak{a}_{\mathbf{q}}$, and $A_{\mathbf{q}}^{+}(P)$ for its exponential. The Langlands decomposition of a given $P \in \mathcal{P}_{\sigma}^{\min }$ is always written as $M A N_{P}$ or $M A N$; then $M A=M_{1}$, and $N_{P}=N$ is as above. Moreover, the maximality of $\mathfrak{a}_{\mathrm{q}}$ implies that $\mathfrak{a}_{\mathrm{q}}=\mathfrak{a} \cap \mathfrak{q}$, where $\mathfrak{a}=\log A$. We denote the real and complex linear dual spaces of $\mathfrak{a}$ and $\mathfrak{a}_{\mathrm{q}}$ by $\mathfrak{a}^{*}, \mathfrak{a}_{\mathfrak{c}}^{*}, \mathfrak{a}_{\mathrm{q}}^{*}$ and $\mathfrak{a}_{\mathrm{q}}^{*}$, respectively, and view the latter two spaces as the subspaces of the former two, consisting of the linear forms which vanish on $\mathfrak{a}_{\mathbf{h}}=\mathfrak{a} \cap \mathfrak{h}$. In particular, the element $\rho=\rho_{P}=\left.\frac{1}{2} \operatorname{tr} \operatorname{ad}(\cdot)\right|_{\mathfrak{n}} \in \mathfrak{a}^{*}$ satisfies $\sigma \theta \rho=\rho$ and hence belongs to $\mathfrak{a}_{\mathrm{q}}^{*}$. The inner product $\langle\cdot, \cdot\rangle$ is transferred to a complex bilinear form on $\mathfrak{a}_{\mathrm{q}}^{*}$ by duality. For $\lambda \in \mathfrak{a}_{\mathbf{c}}^{*}$ and $a \in A$ we write $a^{\lambda}=e^{\lambda(\log a)}$.

The reflection group $W$ of $\Sigma$ is naturally isomorphic to $N_{K}\left(\mathfrak{a}_{\mathrm{q}}\right) / Z_{K}\left(\mathfrak{a}_{\mathrm{q}}\right)$, the normalizer modulo the centralizer of $\mathfrak{a}_{\mathrm{q}}$ in $K$. Let $W_{K \cap H}$ be the canonical image of $N_{K \cap H}\left(\mathfrak{a}_{\mathrm{q}}\right)$ in $W$, then the open double $P \times H$ cosets in $G$ are parametrized by the quotient $W / W_{K \cap H}$, in the obvious way. It will be convenient to fix, once and for all, a set $\mathcal{W}$ of representatives in $N_{K}\left(\mathfrak{a}_{\mathrm{q}}\right)$ for this quotient. It will also be convenient to denote the element of $\mathcal{W}$ which represents $e W_{K \cap H}$ in $W / W_{K \cap H}$ by 1 . We now have the following generalized Cartan decomposition of $G$, for each $Q \in \mathcal{P}_{\sigma}^{\min }$ :

$$
G=\mathrm{cl} \bigcup_{w \in \mathcal{W}} K A_{\mathrm{q}}^{+}(Q) w H
$$

here $\mathrm{cl}$ stands for 'closure', and the union inside $\mathrm{cl}$ is disjoint. The map $(k, h, a) \mapsto$ $k w^{-1} a w h$ is a diffeomorphism of $K \times_{K \cap H \cap M} H \times A_{\mathrm{q}}^{+}(Q)$ onto the open subset $K A_{\mathrm{q}}^{+}(Q) w H$ of $G$. Moreover, $\operatorname{cl} K A_{\mathrm{q}}^{+}(Q) w H=K \mathrm{cl}\left(A_{\mathrm{q}}^{+}(Q)\right) w H$, and if an element $x$ of this set is written $x=k a w h$ according to the latter decomposition, then the $a \in \operatorname{cl} A_{\mathrm{q}}^{+}(Q)$ is unique.

We now recall from [6] the definition of the principal series for $G / H$. Let $\widehat{M}_{\mathrm{fu}}$ denote the set of (equivalence classes of) irreducible finite dimensional unitary representations of $M$. For $P=M A N \in \mathcal{P}_{\sigma}^{\min }$ we consider the representation $\pi_{\xi, \lambda}=\pi_{P, \xi, \lambda}$ of $G$ induced from the representation $\xi \otimes e^{\lambda} \otimes 1$ of $P$, where $\xi \in \widehat{M}_{\mathrm{fu}}$ and $\lambda \in \mathfrak{a}_{\mathrm{qc}}^{*}$. In accordance with loc.cit. we use left induction; thus the space $C^{\infty}(P: \xi: \lambda)=C^{\infty}(\xi: \lambda)$ of smooth vectors for $\pi_{\xi, \lambda}$ is the space of smooth functions $f: G \rightarrow \mathcal{H}_{\xi}$ satisfying the transformation rule

$$
f(\operatorname{man} x)=a^{\lambda+\rho} \xi(m) f(x) \quad(m \in M, a \in A, n \in N, x \in G),
$$

and $G$ acts from the right. Similarly, we denote by $C^{-\infty}(P: \xi: \lambda)=C^{-\infty}(\xi: \lambda)$ the space of generalized functions $f: G \rightarrow \mathcal{H}_{\xi}$ satisfying (6). It will also be useful to work with the compact picture of these representations; it is obtained by taking restrictions to $K$ of the above functions. More precisely, let $C^{\infty}(K: \xi)$ and $C^{-\infty}(K: \xi)$ denote the spaces of smooth, respectively generalized, functions from $K$ to $\mathcal{H}_{\xi}$ satisfying the transformation rule

$$
f(m x)=\xi(m) f(x) \quad\left(m \in K_{\mathrm{M}}, x \in K\right),
$$

where we have written $K_{\mathrm{M}}=K \cap M$. Then restriction to $K$ induces a linear isomorphism $C^{-\infty}(\xi: \lambda) \simeq C^{-\infty}(K: \xi)$, mapping $C^{\infty}(\xi: \lambda)$ onto $C^{\infty}(K: \xi)$. Via this isomorphism we 
transfer the induced representation to a representation of $G$ on $C^{-\infty}(K: \xi)$, again denoted by $\pi_{P, \xi, \lambda}=\pi_{\xi, \lambda}$. We recall that

$$
\langle f \mid g\rangle=\int_{K}\langle f(k) \mid g(k)\rangle d k
$$

defines a sesquilinear pairing $C^{\infty}(K: \xi) \times C^{-\infty}(K: \xi) \rightarrow \mathbb{C}$, which is equivariant for $\pi_{\xi, \lambda}, \pi_{\xi,-\bar{\lambda}}$. In particular, for $\lambda \in i \mathfrak{a}_{\mathrm{q}}^{*}$ the representation $\pi_{\xi, \lambda}$ is unitarizable; the associated Hilbert space is the space $L^{2}(K: \xi)$ of square integrable functions in $C^{-\infty}(K: \xi)$. Here and in the following we use the convention that bilinear pairings are denoted by $\langle\cdot, \cdot\rangle$, whereas sesquilinear pairings are denoted by $\langle\cdot \mid \cdot\rangle$, the latter are always assumed to be skew linear in the second component.

For each $\xi \in \widehat{M}_{\mathrm{fu}}$, let $V(\xi)$ denote the formal direct sum

$$
V(\xi)=\bigoplus_{w \in \mathcal{W}} \mathcal{H}_{\xi}^{w H_{\mathrm{M}} w^{-1}}
$$

provided with the direct sum inner product, where $H_{\mathrm{M}}=M \cap H$, and where $\mathcal{H}_{\xi}^{w H_{\mathrm{M}} w^{-1}}$ is the space of $w H_{\mathrm{M}} w^{-1}$-fixed vectors in $\mathcal{H}_{\xi}$. It will be convenient to write $V(\xi, w)$ for the image of $\mathcal{H}_{\xi}^{w H_{\mathrm{M}} w^{-1}}$ in $V(\xi)$, and $\mathrm{pr}_{w}: \psi \mapsto \psi_{w}$ for the orthogonal projection of $V(\xi)$ onto this component.

Let $\widehat{M}_{H}$ be the set of (equivalence classes of) those $\xi$, for which $V(\xi) \neq 0$. Notice that

$$
M=K_{\mathrm{M}} w H_{\mathrm{M}} w^{-1}
$$

for all $w \in \mathcal{W}$, and hence the representations in $\widehat{M}_{H}$ restrict irreducibly to $K_{\mathrm{M}}$ (see [13], Lemma 1). By [6], Thm. 5.10, the representations $\pi_{\xi, \lambda}$ have non-trivial $H$-fixed distribution vectors for generic $\lambda$, if and only if $\xi \in \widehat{M}_{H}$. For this reason the series of representations $\pi_{\xi, \lambda}$ with $\xi \in \widehat{M}_{H}$ and $\lambda \in \mathfrak{a}_{\mathrm{q}}^{*}$ is called the principal series for $G / H$. In fact, by loc.cit. the space $C^{-\infty}(K: \xi)^{H}$ of $H$-fixed distribution vectors for $\pi_{\xi, \lambda}$ is in bijective correspondence with $V(\xi)$, for generic $\lambda$. The correspondence is given as follows. If $R \in \mathbb{R}$, then we put

$$
\mathfrak{a}_{\mathrm{q}}^{*}(P, R)=\left\{\lambda \in \mathfrak{a}_{\mathrm{qc}}^{*} \mid\langle\operatorname{Re} \lambda, \alpha\rangle<R \quad \text { for } \quad \alpha \in \Sigma(P)\right\} .
$$

Let $\Omega$ be the open subset $\cup_{w \in \mathcal{W}} P w H$ of $G$, and let the linear map $j(\xi: \lambda)=j(P: \xi: \lambda)$ from $V(\xi)$ to $C^{-\infty}(\xi: \lambda)^{H}$ be defined by

$$
j(\xi: \lambda)(\eta)(x)=\left\{\begin{array}{lll}
a^{\lambda+\rho} \xi(m) \eta_{w} & \text { for } & x=\operatorname{man} w h \in \Omega \\
& & (m \in M, a \in A, n \in N, w \in \mathcal{W}, h \in H) ; \\
0 & \text { for } \quad x \notin \Omega .
\end{array}\right.
$$

for $\eta \in V(\xi), \xi \in \widehat{M}_{H}$ and $\lambda \in \mathfrak{a}_{\mathrm{q} c}^{*}$ with $\lambda+\rho \in \mathfrak{a}_{\mathrm{q}}^{*}(P, 0)$ (this condition on $\lambda$ implies that $x \mapsto j(\xi: \lambda)(\eta)(x)$ is continuous $)$. Then the $\operatorname{Hom}\left(V(\xi), C^{-\infty}(K: \xi)\right)$-valued function $\lambda \mapsto j(\xi: \lambda)$ extends meromorphically to $\mathfrak{a}_{\mathrm{q}}^{*}$, and $j(\xi: \lambda)$ is a bijection from $V(\xi)$ onto 
$C^{-\infty}(\xi: \lambda)^{H}$ for generic $\lambda \in \mathfrak{a}_{\mathrm{qc}}^{*}$. In particular, the elements of $C^{-\infty}(\xi: \lambda)^{H}$ are uniquely determined by their restrictions to $\Omega$, for generic $\lambda$.

Let $\mathbb{D}(G / H)$ denote the algebra of invariant differential operators on $G / H$. Let $U(\mathfrak{g})$ denote the universal enveloping algebra of the complexification $\mathfrak{g}_{\mathbf{c}}$ of $\mathfrak{g}$, and $U(\mathfrak{g})^{H}$ the subalgebra of $H$-fixed elements. Recall that the right action $R$ of $G$ on $C^{\infty}(G)$ induces a surjective homomorphism of algebras

$$
r: U(\mathfrak{g})^{H} \rightarrow \mathbb{D}(G / H),
$$

whose kernel is $U(\mathfrak{g})^{H} \cap U(\mathfrak{g}) \mathfrak{h}$. Let $\mathfrak{b}$ be a maximal abelian subspace of $\mathfrak{q}$, containing $\mathfrak{a}_{\mathrm{q}}$, then $\mathfrak{b}$ is a Cartan subspace for $G / H$. Let $\Sigma(\mathfrak{b})$ denote the root system of $\mathfrak{b}$ in $\mathfrak{g}_{c}$ and $W(\mathfrak{b})$ its reflection group. Then there is a natural isomorphism $\gamma$ (called the Harish-Chandra isomorphism) of $\mathbb{D}(G / H)$ onto the algebra $I(\mathfrak{b})=S(\mathfrak{b})^{W(\mathfrak{b})}$ of invariants for $W(\mathfrak{b})$ in $S(\mathfrak{b})$ (the symmetric algebra of $\mathfrak{b}_{\mathfrak{c}}$ ).

Let $P=M A N \in \mathcal{P}_{\sigma}^{\min }$. Since the distributions in $C^{-\infty}(\xi: \lambda)^{H}$ are right $H$-invariant there is a natural action of the algebra $\mathbb{D}(G / H)$ on this space. We shall now discuss this action and at the same time fix some notations.

The space $M_{1} / H_{\mathrm{M}_{1}}$ is again a reductive symmetric space (here $H_{\mathrm{M}_{1}}=M_{1} \cap H$ ); let $\mathbb{D}\left(M_{1} / H_{\mathrm{M}_{1}}\right)$ denote its algebra of invariant differential operators. Then there is a natural map ' $\mu_{P}: \mathbb{D}(G / H) \rightarrow \mathbb{D}\left(M_{1} / H_{\mathrm{M}_{1}}\right)$ defined by the requirement

$$
D-\mu_{P}(D) \in \mathfrak{n} U(\mathfrak{g})+U(\mathfrak{g}) \mathfrak{h} ;
$$

here we have abused notations by identifying an element $D \in \mathbb{D}(G / H)$ with any $X \in$ $U(\mathfrak{g})^{H}$ for which $D=r(X)$ (and similarly for $\mathbb{D}\left(M_{1} / H_{\mathrm{M}_{1}}\right)$ ). It is easily seen that ' $\mu_{P}$ is a homomorphism of algebras.

Define the real analytic function $\left.d_{P}: M_{1} \rightarrow\right] 0, \infty[$ by

$$
d_{P}(m)=\left|\operatorname{det}\left(\left.\operatorname{Ad}(m)\right|_{\mathfrak{n}}\right)\right|^{1 / 2} .
$$

Then the map $D \mapsto d_{P}^{-1} \circ D \circ d_{P}$ defines an automorphism of $\mathbb{D}\left(M_{1} / H_{\mathrm{M}_{1}}\right)$. We define the algebra homomorphism $\mu: \mathbb{D}(G / H) \rightarrow \mathbb{D}\left(M_{1} / H_{\mathrm{M}_{1}}\right)$ by

$$
\mu(D)=d_{P}^{-1} \circ \mu_{P}(D) \circ d_{P} .
$$

Let $W_{\mathrm{M}_{1}}(\mathfrak{b})$ be the Weyl group of $\mathfrak{b}_{\mathfrak{c}}$ in $\mathfrak{m}_{1} \mathfrak{c}$, and let $I_{\mathrm{M}_{1}}(\mathfrak{b})=S(\mathfrak{b})^{W_{\mathrm{M}_{1}}(\mathfrak{b})}$ denote the set of invariants in $S(\mathfrak{b})$ for this Weyl group. Moreover, let $\gamma_{\mathrm{M}_{1}}: \mathbb{D}\left(M_{1} / H_{\mathrm{M}_{1}}\right) \rightarrow I_{\mathrm{M}_{1}}(\mathfrak{b})$ be the corresponding Harish-Chandra isomorphism, then it is easily seen that

$$
\gamma_{\mathrm{M}_{1}} \circ \mu=\gamma
$$

This justifies the notation in the definition (14), since the resulting map $\mu$ does not depend on the particular choice of $P \in \mathcal{P}_{\sigma}^{\min }$.

The space $M_{1} / H_{\mathrm{M}_{1}}$ naturally decomposes as a product $M_{1} / H_{\mathrm{M}_{1}} \simeq M / H_{\mathrm{M}} \times A_{\mathrm{q}}$. This induces a decomposition of the algebra of invariant differential operators:

$$
\mathbb{D}\left(M_{1} / H_{\mathrm{M}_{1}}\right) \simeq \mathbb{D}\left(M / H_{\mathrm{M}}\right) \otimes U\left(\mathfrak{a}_{\mathrm{q}}\right)
$$


Since $U\left(\mathfrak{a}_{\mathrm{q}}\right)$ is naturally isomorphic to the symmetric algebra $S\left(\mathfrak{a}_{\mathrm{q}}\right)$, and hence to the algebra of polynomial functions $\mathfrak{a}_{\mathrm{q} c}^{*} \rightarrow \mathbb{C}$, the elements of $\mathbb{D}\left(M_{1} / H_{\mathrm{M}_{1}}\right)$ may be viewed as $\mathbb{D}\left(M / H_{\mathrm{M}}\right)$-valued polynomial functions on $\mathfrak{a}_{\mathrm{q}}^{*}$. In particular, if $D \in \mathbb{D}(G / H)$, then $\mu_{P}(D)$ and $\mu(D)$ may be viewed as polynomial functions $\mathfrak{a}_{\mathrm{qc}}^{*} \rightarrow \mathbb{D}\left(M / H_{\mathrm{M}}\right)$. We denote by $\mu_{P}(D: \lambda)$ and $\mu(D: \lambda)$ respectively their values in an element $\lambda \in \mathfrak{a}_{\mathrm{q}}^{*}$. It is readily verified that $\mu(D: \lambda)=\mu_{P}\left(D: \lambda+\rho_{P}\right)$.

Let $w \in N_{K}\left(\mathfrak{a}_{\mathrm{q}}\right)$. Then conjugation by $w$ in $U(\mathfrak{g})$ induces an algebra isomorphism $\mathbb{D}\left(M_{1} / H_{\mathrm{M}_{1}}\right) \rightarrow \mathbb{D}\left(M_{1} / w H_{\mathrm{M}_{1}} w^{-1}\right)$, which we denote by $D \mapsto \operatorname{Ad}(w) D$. Thus

$$
\mu_{w}:=\operatorname{Ad}(w) \circ \mu: \mathbb{D}(G / H) \rightarrow \mathbb{D}\left(M_{1} / w H_{\mathrm{M}_{1}} w^{-1}\right) \simeq \mathbb{D}\left(M / w H_{\mathrm{M}} w^{-1}\right) \otimes S\left(\mathfrak{a}_{\mathrm{q}}\right)
$$

is a homomorphism of algebras. If $D \in \mathbb{D}(G / H), \lambda \in \mathfrak{a}_{\mathrm{q}}^{*}$, then the operator $\mu_{w}(D: \lambda)$ in $\mathbb{D}\left(M / w H_{\mathrm{M}} w^{-1}\right)$ is defined as the evaluation of $\mu_{w}(D)$ in $\lambda$.

Let $w \in \mathcal{W}$. Then via $\xi$ we have a natural action of the algebra $\mathbb{D}\left(M / w H_{\mathrm{M}} w^{-1}\right)$ on the space $V(\xi, w) \simeq \mathcal{H}_{\xi}^{w H_{\mathrm{M}} w^{-1}}$. Let $\xi_{w}: \mathbb{D}\left(M / w H_{\mathrm{M}} w^{-1}\right) \rightarrow \operatorname{End}(V(\xi, w))$ be the associated homomorphism of algebras. If $D \in \mathbb{D}(G / H), \lambda \in \mathfrak{a}_{\mathrm{qc}}^{*}$, then we define $\mu(D: \xi: \lambda) \in$ End $(V(\xi))$ as the direct sum of the endomorphisms $\xi_{w}\left(\mu_{w}(D: \lambda)\right) \in \operatorname{End}(V(\xi, w)), w \in$ $\mathcal{W}$.

We now recall from [13], eqn. (38), that for every $D \in \mathbb{D}(G / H)$ we have

$$
D(j(\xi: \lambda) \eta)=j(\xi: \lambda)(\mu(D: \xi: \lambda) \eta) \quad(\eta \in V(\xi)),
$$

as a meromorphic identity in $\lambda \in \mathfrak{a}_{\mathrm{q}}^{*}$.

If $D$ belongs to $\mathbb{Z}(G / H)=r(\mathfrak{Z})$, the canonical image in $\mathbb{D}(G / H)$ of the center $\mathfrak{Z}$ of $U(\mathfrak{g})$, then $\mu(D: \xi: \lambda)$ can be computed as follows.

Let $\mathfrak{j}$ be a Cartan subalgebra of $\mathfrak{g}$ containing $\mathfrak{a}_{\mathrm{q}}$, and let $\mathfrak{j}_{0}$ denote the orthocomplement of $\mathfrak{a}_{\mathrm{q}}$ in $\mathfrak{j}$. Then

$$
\mathfrak{j}=\mathfrak{j}_{0} \oplus \mathfrak{a}_{\mathrm{q}} .
$$

Via this decomposition we view $\mathfrak{j}_{0 \mathbf{c}}^{*}$ and $\mathfrak{a}_{\mathrm{q}}^{*}$ as subspaces of $\mathfrak{j}_{\mathbf{c}}^{*}$. Let $W(\mathfrak{j})$ denote the Weyl group of the root system $\Sigma(\mathfrak{j})$ of $\mathfrak{j}_{\mathbf{c}}$ in $\mathfrak{g}_{\mathbf{c}}$, and let $\gamma_{\mathfrak{j}}^{\mathfrak{g}}$ denote the Harish-Chandra isomorphism from $\mathfrak{Z}$ onto $I(\mathfrak{j}):=S(\mathfrak{j})^{W(\mathfrak{j})}$. Let $\Lambda_{\xi} \in \mathbf{j}_{0 \mathbf{c}}^{*}$ denote the infinitesimal character of $\xi$; here we use the decomposition $\mathfrak{j}_{0}=(\mathfrak{j} \cap \mathfrak{m}) \oplus(\mathfrak{a} \cap \mathfrak{h})$ to identify $(\mathfrak{j} \cap \mathfrak{m})_{\mathbf{c}}^{*}$ with a subspace of $\mathfrak{j}_{0 \boldsymbol{c}}^{*}$. Then $\Lambda_{\xi}+\lambda$ is the infinitesimal character of the induced representation $\pi_{\xi, \lambda}$, that is, if $Z \in \mathfrak{Z}$, then $Z$ acts by the scalar $\gamma_{j}^{\mathfrak{g}}\left(Z: \Lambda_{\xi}+\lambda\right)$ on $\pi_{\xi, \lambda}$. Performing the substitution $D=r(Z)$ in (18) and using the injectivity of $j(\xi: \lambda)$ for generic $\lambda \in \mathfrak{a}_{\mathrm{qc}}^{*}$, we infer, for all $Z \in \mathfrak{J}, \lambda \in \mathfrak{a}_{\mathrm{q}}^{*}$, that

$$
\mu(r(Z): \xi: \lambda)=\gamma_{j}^{\mathfrak{g}}\left(Z: \Lambda_{\xi}+\lambda\right) I_{V(\xi)} .
$$

\section{$3 \quad$ Normalization of measures}

In this section, we shall specify the normalization of the various measures involved in our Plancherel formula. From now on $d x=d(g H)$ will be a fixed choice of invariant 
measure on $G / H$, and $d k$ the normalized Haar measure of $K$. Let $P \in \mathcal{P}_{\sigma}^{\text {min }}$. If $d a$ is an invariant measure on $A_{\mathrm{q}}$, the decomposition (5) induces the following formula for $d x$ (see [37], p. 149):

$$
\int_{G / H} f(x) d x=C \int_{K} \int_{A_{\mathrm{q}}^{+}(P)} \sum_{w \in \mathcal{W}} f(k a w H) J\left(w^{-1} a w\right) d a d k
$$

for $f \in C_{c}(G / H)$, where $C$ is a positive constant and $J$ is the function

$$
J(a)=\prod_{\alpha \in \Sigma(P)}\left|a^{\alpha}-a^{-\alpha}\right|^{m_{\alpha}^{+}}\left[a^{\alpha}+a^{-\alpha}\right]^{m_{\alpha}^{-}}
$$

on $A_{\mathrm{q}}$. Here the numbers $m_{\alpha}^{ \pm}$are defined as follows: For $\alpha \in \Sigma$ let $\mathfrak{g}_{\alpha}=\mathfrak{g}_{\alpha}^{+} \oplus \mathfrak{g}_{\alpha}^{-}$denote the decomposition of the root space $\mathfrak{g}_{\alpha}$ into the \pm 1 eigenspaces for $\sigma \theta$, then $m_{\alpha}^{ \pm}=\operatorname{dim} \mathfrak{g}_{\alpha}^{ \pm}$. Notice that $J$ is independent of the choice of $P$. The constant $C$ is also independent of the choice of $P$; this follows from the observation that the action of $W$ is transitive on $\mathcal{P}_{\sigma}^{\min }$ and leaves the measure $d a$ invariant. We shall use the normalization of $d a$ for which $C=1$.

Let $|W|$ be the order of $W$, and let $d \lambda$ be Lebesgue measure on $i \mathfrak{a}_{\mathrm{q}}^{*}$, normalized as $|W|^{-1}$ times the regularly normalized measure $d \mu_{\text {reg }}(\lambda)$; the latter measure is the one which allows an extension of the classical Fourier transform $\varphi \mapsto \hat{\varphi}, C_{c}\left(A_{\mathrm{q}}\right) \rightarrow C\left(i \mathfrak{a}_{\mathrm{q}}^{*}\right)$, defined by the formula

$$
\hat{\varphi}(\lambda)=\int_{A_{\mathrm{q}}} \varphi(a) a^{-\lambda} d a,
$$

to an isometry from $L^{2}\left(A_{\mathrm{q}}, d a\right)$ onto $L^{2}\left(i \mathfrak{a}_{\mathrm{q}}^{*}, d \mu_{\mathrm{reg}}\right)$.

If $w \in N_{K}\left(\mathfrak{a}_{\mathrm{q}}\right)$ then the space $M / w H_{\mathrm{M}} w^{-1}$ is compact by (10). We equip it with the $M$-invariant measure for which this space has total measure one.

If $P_{1}=M A N_{1}, P_{2}=M A N_{2}$ are $\sigma$-minimal parabolic subgroups with the indicated Langlands decompositions, let the Haar measure of the nilpotent group $\bar{N}_{1} \cap N_{2}$ be normalized as in [33], $\S 2$. The sole reason for using this normalization is to make valid the usual product formulas for standard intertwining operators (cf. [6], Prop. 4.6). If $P \in \mathcal{P}_{\sigma}^{\text {min }}$, we let $d \bar{n}$ denote the Haar measure of $\bar{N}_{P}$ (normalized according to the above), and define the positive constant $c\left(A_{\mathrm{q}}\right)$ by (see also [7], eqn. (122)):

$$
c\left(A_{\mathbf{q}}\right)^{-1}=\int_{\bar{N}_{P}} e^{2 \rho_{P} H_{P}(\bar{n})} d \bar{n} .
$$

Here $H_{P}: G \rightarrow \mathfrak{a}$ is the real analytic map defined by $x \in N_{P} \exp H_{P}(x) M K$. Notice that the constant $c\left(A_{\mathrm{q}}\right)$ is independent of the choice of $P \in \mathcal{P}_{\sigma}^{\min }$.

\section{The Fourier transform}

Let $P \in \mathcal{P}_{\sigma}^{\min }$, and let $\xi \in \widehat{M}_{H}$. As a meromorphic $\operatorname{Hom}\left(V(\xi), C^{-\infty}(K: \xi)\right)$-valued function on $\mathfrak{a}_{\mathrm{q}}^{*}$ the function $\lambda \mapsto j(\xi: \lambda)$ may have singularities at points of $i \mathfrak{a}_{\mathrm{q}}^{*}$. Following 
[13] we shall now introduce a suitable normalization of the $j(\xi: \lambda)$ to get rid of these singularities.

If $P_{1}, P_{2} \in \mathcal{P}_{\sigma}^{\min }$, let $A\left(P_{2}: P_{1}: \xi: \lambda\right)$ denote the standard intertwining operator from $\pi_{P_{1}, \xi, \lambda}$ to $\pi_{P_{2}, \xi, \lambda}$, defined as in [6]. This operator maps $C^{-\infty}\left(P_{1}: \xi: \lambda\right)$ equivariantly and continuous linearly to $C^{-\infty}\left(P_{2}: \xi: \lambda\right)$, and depends meromorphically on $\lambda$ as an endomorphism of $C^{-\infty}(K: \xi)$ ('the compact picture', cf. [6], Prop. 4.11). Since the standard intertwining operators are bijective for generic $\lambda$, the map

$$
j^{\circ}(P: \xi: \lambda):=c\left(A_{\mathbf{q}}\right)^{-1} A(\bar{P}: P: \xi: \lambda)^{-1} j(\bar{P}: \xi: \lambda)
$$

is again a bijection from $V(\xi)$ onto $C^{-\infty}(P: \xi: \lambda)^{H}$ for generic $\lambda \in \mathfrak{a}_{\mathrm{q} c}^{*}$ (see (23) for the definition of $c\left(A_{\mathrm{q}}\right)$ ).

Remark 4.1 Note that the intertwining operator $A(\bar{P}: P: \xi: \lambda)$ depends on the chosen normalization of the Haar measure $d \bar{n}$ of $\bar{N}_{P}$. However, the operator $c\left(A_{\mathrm{q}}\right) A(\bar{P}: P: \xi: \lambda)$ is independent of this particular normalization. Therefore the map $j^{\circ}(\xi: \lambda)$ is independent of the chosen normalization of $d \bar{n}$ as well.

By meromorphy of the inverted intertwining operator (see [6]), the expression (24) is meromorphic as a $\operatorname{Hom}\left(V(\xi), C^{-\infty}(K: \xi)\right)$-valued function of $\lambda \in \mathfrak{a}_{\mathrm{q} c}^{*}$. Notice that eqn. (18) remains valid if $j$ is replaced by $j^{\circ}$, because of the equivariance of the intertwining operator.

If $\epsilon>0$, we define the subset $\mathfrak{a}_{\mathrm{q}}^{*}(\epsilon)$ of $\mathfrak{a}_{\mathrm{q} c}^{*}$ by:

$$
\mathfrak{a}_{\mathrm{q}}^{*}(\epsilon)=\left\{\lambda \in \mathfrak{a}_{\mathrm{q}}^{*}|\quad|\langle\operatorname{Re} \lambda, \alpha\rangle \mid<\epsilon \quad \text { for all } \quad \alpha \in \Sigma\right\} .
$$

Notice that $\mathfrak{a}_{\mathrm{q}}^{*}(\epsilon)=\mathfrak{a}_{\mathrm{q}}^{*}(P, \epsilon) \cap \mathfrak{a}_{\mathrm{q}}^{*}(\bar{P}, \epsilon)$.

According to [13] we now have the following regularity result.

Theorem 4.2 Let $\xi \in \widehat{M}_{H}$. Then there exists a constant $\epsilon>0$ such that the meromorphic $\operatorname{Hom}\left(V(\xi), C^{-\infty}(K: \xi)\right)$-valued function $\lambda \mapsto j^{\circ}(\xi: \lambda)$ is regular on the neighborhood $\mathfrak{a}_{\mathrm{q}}^{*}(\epsilon)$ of $i \mathfrak{a}_{\mathrm{q}}^{*}$.

Theorem 4.2 allows us to define a Fourier transform. At a later stage we shall have to keep track of particular choices of representations; therefore we shall distinguish between irreducible finite dimensional unitary representations of $M$ and their equivalence classes.

In this spirit we shall write $[\xi] \in \widehat{M}_{\mathrm{fu}}$, respectively $\xi \in \omega \in \widehat{M}_{\mathrm{fu}}$, to indicate that $\xi$ is a finite dimensional irreducible unitary representation of $M$, whose equivalence class belongs to $\widehat{M}_{\mathrm{fu}}$, respectively equals $\omega \in \widehat{M}_{\mathrm{fu}}$. Moreover, we write $\mathcal{H}_{\xi}$ for the finite dimensional complex Hilbert space in which $\xi$ is (unitarily) realized, and we write

$$
d_{\omega}=d_{\xi}=\operatorname{dim} \mathcal{H}_{\xi}
$$

We now come to the definition of a Fourier transform on $C_{c}^{\infty}(G / H)$. For $[\xi] \in \widehat{M}_{H}$, let $L^{2}(K: \xi)$ denote the Hilbert space of square integrable elements in $C^{-\infty}(K: \xi)$ (cf. Section 2). We equip the dual $V(\xi)^{*}$ of $V(\xi)$ with the dual Hermitean inner product, and $L^{2}(K: \xi) \otimes V(\xi)^{*}$ with the tensor product inner product. 
If $f \in C_{c}^{\infty}(G / H)$, then for $[\xi] \in \widehat{M}_{H}, \lambda \in i \mathfrak{a}_{\mathrm{q}}^{*}$ we define the element

$$
\hat{f}(\xi: \lambda)=\hat{f}_{P}(\xi: \lambda) \in \operatorname{Hom}\left(V(\xi), C^{-\infty}(K: \xi)\right) \simeq C^{-\infty}(K: \xi) \otimes V(\xi)^{*}
$$

by

$$
\begin{aligned}
\hat{f}(\xi: \lambda) & =\left[\pi_{\xi,-\lambda}(f) \times I\right] j^{\circ}(P: \xi:-\lambda) \\
& :=\int_{G / H} f(g H)\left[\pi_{\xi,-\lambda}(g) \otimes I\right] j^{\circ}(P: \xi:-\lambda) d(g H) .
\end{aligned}
$$

Here we view $j^{\circ}(P: \xi: \lambda)$ as an element of $C^{-\infty}(K: \xi) \otimes V(\xi)^{*}$, and $\pi_{\xi,-\lambda}=\pi_{P, \xi,-\lambda}$ denotes the principal series representation $\operatorname{Ind}_{P}^{G}(\xi \otimes-\lambda \otimes 1)$, realized on $C^{-\infty}(K: \xi)$ (cf. Section 2).

By definition we thus have that $\hat{f}(\xi: \lambda) \in C^{-\infty}(K: \xi)$ for $\lambda \in i \mathfrak{a}_{\mathrm{q}}^{*}$. However, it is easily seen that actually $\hat{f}(\xi: \lambda) \in C^{\infty}(K: \xi)$ (use Lemma 4.3 below to throw differentiations on $f)$. Moreover, the map $f \mapsto \hat{f}(\xi: \lambda)$ is continuous from $C_{c}^{\infty}(G / H)$ to $C^{\infty}(K: \xi)$, for all $\lambda \in i \mathfrak{a}_{\mathrm{q}}^{*}$.

The following intertwining property is obvious from the definitions:

Lemma 4.3 Let $f \in C_{c}^{\infty}(G / H),[\xi] \in \widehat{M}_{H}, \lambda \in i \mathfrak{a}_{\mathrm{q}}^{*}$. Then

$$
\widehat{L_{x} f}(\xi: \lambda)=\left[\pi_{\xi,-\lambda}(x) \otimes I\right] \hat{f}(\xi: \lambda) \quad(x \in G) .
$$

\section{$5 \quad$ Eisenstein integrals}

Throughout this paper $\tau$ will be a unitary representation of $K$ in a finite dimensional Hilbert space $V_{\tau}$. A function $f: G / H \rightarrow V_{\tau}$ is called $\tau$-spherical if it transforms according to the rule:

$$
f(k x)=\tau(k) f(x) \quad(k \in K, x \in G / H) .
$$

The space of smooth $\tau$-spherical functions $G / H \rightarrow V_{\tau}$ is denoted by $C^{\infty}(G / H: \tau)$.

Let $\tau_{\mathrm{M}}$ denote the restriction of $\tau$ to $K_{\mathrm{M}}$. If $w \in \mathcal{W}$, then by $C^{\infty}\left(M / w H_{\mathrm{M}} w^{-1}: \tau_{\mathrm{M}}\right)$ we denote the space of smooth functions $\varphi: M / w H_{\mathrm{M}} w^{-1} \rightarrow V_{\tau}$ which are $\tau_{\mathrm{M}^{-}}$spherical, i.e. transform according to the rule

$$
\varphi(k m)=\tau(k) \varphi(m) \quad\left(k \in K_{\mathrm{M}}, m \in M / w H_{\mathrm{M}} w^{-1}\right) .
$$

We recall from Section 3 that $M / w H_{\mathrm{M}} w^{-1}$ is equipped with an invariant measure for which it has total measure 1 . In view of (10) the space $C^{\infty}\left(M / w H_{\mathrm{M}} w^{-1}: \tau_{\mathrm{M}}\right)$ is finite dimensional; it may be viewed as a subspace of $L^{2}\left(M / w H_{\mathrm{M}} w^{-1}\right) \otimes V_{\tau}$ and thus inherits a Hilbert space structure.

We now define the following formal direct sum of Hilbert spaces:

$$
{ }^{\circ} \mathcal{C}(\tau)=\bigoplus_{w \in \mathcal{W}} C^{\infty}\left(M / w H_{\mathrm{M}} w^{-1}: \tau_{\mathrm{M}}\right)
$$


Note that the space ${ }^{\circ} \mathcal{C}(\tau)$ is finite dimensional. The component associated with $w \in \mathcal{W}$ will be denoted by ${ }^{\circ} \mathcal{C}_{w}(\tau)$, and the projection map onto this component by $\operatorname{pr}_{w}: \psi \mapsto \psi_{w}$.

If $[\xi] \in \widehat{M}_{\mathrm{fu}}$, we denote by $C_{\xi}^{\infty}\left(M / w H_{\mathrm{M}} w^{-1}\right)\left(\right.$ or $\left.C_{[\xi]}^{\infty}\left(M / w H_{\mathrm{M}} w^{-1}\right)\right)$ the space of left $M$-isotypical functions of type $[\xi]$ in $C^{\infty}\left(M / w H_{\mathrm{M}} w^{-1}\right)$. It is easily seen from (10) that this space is finite dimensional. We write ${ }^{\circ} \mathcal{C}_{w, \xi}(\tau)={ }^{\circ} \mathcal{C}_{w}(\tau) \cap\left[C_{\xi}^{\infty}\left(M / w H_{\mathrm{M}} w^{-1}\right) \otimes V_{\tau}\right]$ and denote the direct sum of these over $w \in \mathcal{W}$ by ${ }^{\circ} \mathcal{C}_{\xi}(\tau)$. It follows from the Frobenius reciprocity theorem (for details, see [13], Lemma 3) that ${ }^{\circ} \mathcal{C}_{\xi}(\tau)$ is non-zero if and only if $[\xi] \in \widehat{M}_{H}$ and $\left.\xi^{\vee}\right|_{K_{\mathrm{M}}}$ occurs in the decomposition of $\tau_{\mathrm{M}}$ into irreducible $K_{\mathrm{M}}$-types, where $\xi^{\vee}$ denotes the representation contragradient to $\xi$. We use the notation $\xi^{\vee} \tau$ (or $\left[\xi^{\vee} \uparrow \uparrow \tau\right)$ to indicate this occurrence, and we thus have the finite sum decomposition

$$
{ }^{\circ} \mathcal{C}(\tau)=\bigoplus_{\left[\xi^{\vee}\right] \uparrow}{ }^{\circ} \mathcal{C}_{\xi}(\tau)
$$

where the sum extends over the equivalence classes $[\xi] \in \widehat{M}_{\text {fu }}$ for which $\left[\xi^{\vee}\right] \uparrow \tau$.

We are now ready to define the $\tau$-spherical Eisenstein integral. It will depend on parameters $\psi \in{ }^{\circ} \mathcal{C}(\tau)$ and $\lambda \in \mathfrak{a}_{\mathrm{q} c}^{*}$. Let $P \in \mathcal{P}_{\sigma}^{\min }$. If $\psi \in{ }^{\circ} \mathcal{C}(\tau)$, then for $\lambda \in \mathfrak{a}_{\mathrm{qc}}^{*}$ we define the function $\tilde{\psi}(\lambda: \cdot): G / H \rightarrow V_{\tau}$ by

$$
\tilde{\psi}(\lambda: x)= \begin{cases}a^{\lambda+\rho_{P}} \psi_{w}(m) & \text { for } x=\operatorname{man} w H \\ & (m \in M, a \in A, n \in N, w \in \mathcal{W}) \\ 0 & \text { for } x \notin \cup_{w \in \mathcal{W}} P w H\end{cases}
$$

It follows from [6], Prop. 5.6, that if $\lambda+\rho_{P} \in \mathfrak{a}_{\mathrm{q}}^{*}(P, 0)$ (see $\left.(11)\right)$ then $\tilde{\psi}(\lambda)$ is continuous on $G$. The $\tau$-spherical Eisenstein integral is then defined by

$$
E(\psi: \lambda)(x)=E_{\tau}(P: \psi: \lambda)(x)=\int_{K} \tau(k)^{-1} \tilde{\psi}(\lambda: k x) d k
$$

for $x \in G / H$. Then $\psi \mapsto E(\psi: \lambda)$ is a linear map from ${ }^{\circ} \mathcal{C}(\tau)$ to $C^{\infty}(G / H: \tau)$. Moreover the map $\lambda \mapsto E(\psi: \lambda)$ allows a meromorphic extension to all of $\mathfrak{a}_{\mathrm{qc}}^{*}$ (see [7] and [13]).

Remark 5.1 The present definition of the Eisenstein integral slightly extends the one given in [7], §3. If $\vartheta \subset \widehat{K}$ is a finite set of $K$-types, then we write $\mathbf{V}_{\vartheta}:=C(K)_{\vartheta} \vee$ for the space of continuous functions on $K$ which behave finitely under the right regular representation $R$, with $K$-types contained the set $\vartheta^{\vee}:=\left\{\delta^{\vee} \mid \delta \in \vartheta\right\}$, where $\delta^{\vee} \in \widehat{K}$ denotes the representation contragradient to $\delta$. Moreover, we write

$$
\tau_{\vartheta}:=\left.R\right|_{\vartheta}
$$

for the restriction of $R$ to $\mathbf{V}_{\vartheta}$. If we take $\left(\tau, V_{\tau}\right)=\left(\tau_{\vartheta}, \mathbf{V}_{\vartheta}\right)$ in the present definition of the Eisenstein integral, then we obtain the definition of [7].

The more general $\tau$-spherical Eisenstein integral can be expressed in terms of the more restricted one in a natural way. This allows a straightforward extension of the theory of [7] to the more general Eisenstein integral. For details we refer the reader to [13]. In the 
present paper we shall often refer to [7] for a result on Eisenstein integrals and then apply it to the present more general setting.

We now recall some properties of the singular set of the meromorphic function $\lambda \mapsto$ $E(\psi: \lambda)$ that will be of importance in this paper.

As in [7], if $S \subset \mathfrak{a}_{\mathrm{q} c}^{*} \backslash\{0\}$, let $\Pi_{\mathrm{S}}\left(\mathfrak{a}_{\mathrm{q}}\right)$ be the set of elements of $S\left(\mathfrak{a}_{\mathrm{q}}\right) \backslash\{0\}$ which can be written as a product of linear factors of the form $\langle\xi, \cdot\rangle+c$, with $\xi \in \mathrm{S}, c \in \mathbb{C}$. It is to be understood that $1 \in \Pi_{\mathrm{S}}\left(\mathfrak{a}_{\mathrm{q}}\right)$. Notice that $\mathbb{C}^{*} \Pi_{\mathrm{S}}\left(\mathfrak{a}_{\mathrm{q}}\right)$ is precisely the set of polynomial functions on $\mathfrak{a}_{\mathrm{q}}^{*}$ whose zero locus is contained in a finite union of hyperplanes which are translates of the linear subspaces $\xi^{\perp}, \xi \in \mathrm{S}$. If $\mathrm{S}=\mathfrak{a}_{\mathrm{qc}}^{*} \backslash\{0\}$ we simply write $\Pi\left(\mathfrak{a}_{\mathrm{q}}\right)$ for $\Pi_{\mathrm{S}}\left(\mathfrak{a}_{\mathrm{q}}\right)$.

We now have the following, by [7], Prop. 10.3 (and the above remark). For every $R \in \mathbb{R}$ there exists a polynomial $p \in \Pi_{\Sigma}\left(\mathfrak{a}_{\mathrm{q}}\right)$ such that for every $\psi \in{ }^{\circ} \mathcal{C}(\tau)$ the $C^{\infty}(G / H: \tau)$ valued meromorphic function

$$
E_{p}(P: \psi: \cdot): \lambda \mapsto p(\lambda) E(P: \psi: \lambda)
$$

is regular on $\mathfrak{a}_{\mathrm{q}}^{*}(P, R)$.

Thus the Eisenstein integral has its singularities in a locally finite union of translates of the hyperplanes $\alpha^{\perp}, \alpha \in \Sigma$. It may have singularities at points $\lambda \in i \mathfrak{a}_{\mathrm{q}}^{*}$. We will describe a normalization procedure to obtain Eisenstein integrals which are regular at $i \mathfrak{a}_{\mathrm{q}}^{*}$. To prepare for this we first need to describe the asymptotic behavior of Eisenstein integrals.

Let $Q \in \mathcal{P}_{\sigma}^{\min }$. Then according to [7], $\S 14$, and [13], $\S 4$, there exist unique meromorphic End $\left({ }^{\circ} \mathcal{C}(\tau)\right)$-valued functions $C_{Q \mid P}(s: \cdot)$ on $\mathfrak{a}_{\mathrm{q} c}^{*}(s \in W)$ such that for generic $\lambda \in i \mathfrak{a}_{\mathrm{q}}^{*}$ we have:

$$
E(P: \psi: \lambda)(\text { maw }) \sim \sum_{s \in W} a^{s \lambda-\rho_{Q}}\left[\operatorname{pr}_{w} C_{Q \mid P}(s: \lambda) \psi\right](m) \quad \text { as } \quad a \stackrel{Q}{\longrightarrow} \infty,
$$

for all $\psi \in{ }^{\circ} \mathcal{C}(\tau), w \in \mathcal{W}, m \in M$. Here the relation $\sim$ means that the difference is $o\left(a^{-\rho_{Q}}\right)$ as $a \stackrel{Q}{\longrightarrow} \infty$, and the latter notion means that $\log a$ tends radially to infinity in the open chamber $\mathfrak{a}_{\mathrm{q}}^{+}(Q)$. The endomorphisms $C_{Q \mid P}(s: \lambda)(s \in W)$ are invertible for generic $\lambda \in \mathfrak{a}_{\mathrm{q}}^{*}$. We now define the normalized $\tau$-spherical Eisenstein integral by

$$
E^{\circ}(P: \psi: \lambda)=E\left(P: C_{P \mid P}(1: \lambda)^{-1} \psi: \lambda\right),
$$

for $\psi \in{ }^{\circ} \mathcal{C}(\tau)$, and as a meromorphic identity in $\lambda \in \mathfrak{a}_{\mathrm{q} c}^{*}$. For generic $\lambda \in i \mathfrak{a}_{\mathrm{q}}^{*}$ its asymptotic behavior along the open chambers in the Cartan decomposition is described as above, but with normalized $C$-functions:

$$
E^{\circ}(P: \psi: \lambda)(\text { maw }) \sim \sum_{s \in W} a^{s \lambda-\rho_{Q}}\left[\operatorname{pr}_{w} C_{Q \mid P}^{\circ}(s: \lambda) \psi\right](m) \quad \text { as } \quad a \stackrel{Q}{\longrightarrow} \infty .
$$

Here the normalized $C$-functions are given by:

$$
C_{Q \mid P}^{\circ}(s: \lambda)=C_{Q \mid P}(s: \lambda) C_{P \mid P}(1: \lambda)^{-1} \text {. }
$$


Remark 5.2 A different normalization of the Eisenstein integral was defined in [7], $§ 16$, by the formula: $E^{1}(P: \psi: \lambda):=E\left(P: C_{\bar{P} \mid P}(1: \lambda)^{-1} \psi: \lambda\right)$. This definition extends to the present situation, see Remark 5.1. The associated normalized $C$-functions are given by: $C_{Q \mid P}^{1}(s: \lambda)=C_{Q \mid P}(s: \lambda) C_{\bar{P} \mid P}(1: \lambda)^{-1}$. According to [13], eqn. (52) and the line following eqn. (55), we have, for $P, Q \in \mathcal{P}_{\sigma}^{\min }, \psi \in{ }^{\circ} \mathcal{C}(\tau)$ and $s \in W$ :

$$
E^{\circ}(P: \psi: \lambda)=E^{1}(\bar{P}: \psi: \lambda), \quad C_{Q \mid P}^{\circ}(s: \lambda) \psi=C_{Q \mid \bar{P}}^{1}(s: \lambda) \psi
$$

as meromorphic identities in the variable $\lambda \in \mathfrak{a}_{\mathrm{qc}}^{*}$. The results of [7] for $E^{1}$ and $C^{1}$ are easily translated to results for $E^{\circ}$ and $C^{\circ}$ because of these relations.

For every $R \in \mathbb{R}$ there exists a $p \in \Pi_{\Sigma}\left(\mathfrak{a}_{\mathrm{q}}\right)$ such that the function

$$
E_{p}^{\circ}(P: \psi: \cdot): \lambda \mapsto p(\lambda) E^{\circ}(P: \psi: \lambda)
$$

is regular on $\mathfrak{a}_{\mathrm{q}}^{*}(\bar{P}, R)$, in analogy with $(31)$; see [7], Cor. 16.2 and Prop. 10.3.

We now have the following crucial result, due to [7] and [8] (see also [13], Prop. 5).

Proposition 5.3 Let $P, Q \in \mathcal{P}_{\sigma}^{\min }, s \in W$. Then

$$
C_{Q \mid P}^{\circ}(s:-\bar{\lambda})^{*} C_{Q \mid P}^{\circ}(s: \lambda)=I
$$

as a meromorphic identity in $\lambda \in \mathfrak{a}_{\mathrm{qc}}^{*}$. In particular the normalized $C$-function $C_{Q \mid P}^{\circ}(s: \lambda)$ is a unitary endomorphism of ${ }^{\circ} \mathcal{C}(\tau)$, for every $\lambda \in i \mathfrak{a}_{\mathrm{q}}^{*}$.

The above result implies that the right-hand side of (32) is regular for $\lambda \in i \mathfrak{a}_{\mathrm{q}}^{*}$. Starting from this observation, the following result is obtained in [13], Thm. 2, where it plays a crucial role in the proof of Theorem 4.2 above (conversely it is a consequence of the latter theorem and eqn. (35) below).

Proposition 5.4 Let $\tau$ be a finite dimensional unitary representation of $K$. There exists $\epsilon>0$ such that the meromorphic $C^{\infty}(G / H: \tau)$-valued map $\lambda \mapsto E^{\circ}(\psi: \lambda)$ is regular on $\mathfrak{a}_{\mathrm{q}}^{*}(\epsilon)$ for every $\psi \in{ }^{\circ} \mathcal{C}(\tau)$.

Remark 5.5 Combining the above proposition with Corollary 2 of [13], we now see that (32) is in fact valid for all $\lambda \in i \mathfrak{a}_{\mathrm{q}}^{*}$.

We shall now describe the relation of the Eisenstein integral to the principal series of $G / H$, meanwhile fixing notations that will also be needed at a later stage. If $\vartheta \subset \widehat{K}$ is a finite subset, then we write

$$
\mathcal{H}_{\xi, \vartheta}:=C^{\infty}(K: \xi)_{\vartheta}
$$

for the (finite dimensional) space of $K$-finite functions in $C^{\infty}(K: \xi)$ all of whose $K$ types belong to $\vartheta$. If $[\xi] \in \widehat{M}_{H}, w \in \mathcal{W}$, we shall write $\overline{V(\xi)}$ and $\overline{V(\xi, w)}$ for the Hilbert spaces which are conjugate to $V(\xi)$ and $V(\xi, w)$ respectively. If $T=\varphi \otimes \eta$ belongs to 
$\mathcal{H}_{\xi, \vartheta} \otimes \overline{V(\xi, w)}$, then according to $[7], \S 4$, we may define a function $\psi_{T} \in{ }^{\circ} \mathcal{C}_{w}\left(\tau_{\vartheta}\right)=$ $C^{\infty}\left(M / w H_{\mathrm{M}} w^{-1}: \tau_{\vartheta \mathrm{M}}\right)$ by

$$
\psi_{T}(m)(k)=\left\langle\varphi\left(k^{-1}\right), \xi(m) \eta\right\rangle_{\xi} \quad(m \in M, k \in K) .
$$

The map $T \mapsto \psi_{T}$ is linearly extended to a map $\mathcal{H}_{\xi, \vartheta} \otimes \overline{V(\xi)} \rightarrow{ }^{\circ} \mathcal{C}\left(\tau_{\vartheta}\right)$. Via the Hermitean inner product on $V(\xi)$ we have a natural isometry

$$
\overline{V(\xi)} \simeq V(\xi)^{*}
$$

and accordingly we view $T \mapsto \psi_{T}$ as a linear map from $\mathcal{H}_{\xi, \vartheta} \otimes V(\xi)^{*}$ to ${ }^{\circ} \mathcal{C}\left(\tau_{\vartheta}\right)$. According to $[7]$, Lemma 4.1, we now have:

Lemma 5.6 The map $T \mapsto d_{\xi}^{1 / 2} \psi_{T}$ is an isometry from $\mathcal{H}_{\xi, \vartheta} \otimes V(\xi)^{*}$ onto ${ }^{\circ} \mathcal{C}_{\xi}\left(\tau_{\vartheta}\right)$, for each $[\xi] \in \widehat{M}_{H}$.

According to [13], eqn. (53), the relation with the principal series can now be described as follows. Let $T=\varphi \otimes \eta \in \mathcal{H}_{\xi, \vartheta} \otimes \overline{V(\xi)}$. Then

$$
E_{\tau_{\vartheta}}^{\circ}\left(\psi_{T}: \lambda\right)(x H)(k)=\left\langle\varphi \mid \pi_{\xi, \bar{\lambda}}(k x) j^{\circ}(\xi: \bar{\lambda}) \eta\right\rangle \quad(x \in G, k \in K)
$$

as a meromorphic identity in $\lambda \in \mathfrak{a}_{\mathrm{q}}^{*}$. The sesquilinear pairing in this formula is defined by $(8)$.

We now return to the more general situation of an arbitrary $\tau$. In this case a relation similar to (35) is given in [13], Prop. 12 (we shall however not be using it here). The $\tau$-spherical Eisenstein integrals as well as their normalizations are $\mathbb{D}(G / H)$-finite. The action of the algebra of invariant differential operators may be described as follows. For $w \in \mathcal{W}$ let $\mu_{w}: \mathbb{D}(G / H) \rightarrow \mathbb{D}\left(M_{1} / w H_{\mathrm{M}_{1}} w^{-1}\right)$ be the homomorphism of algebras defined in Section 2. We recall that elements of $\mathbb{D}\left(M_{1} / w H_{\mathrm{M}_{1}} w^{-1}\right)$ may be viewed as $\mathbb{D}\left(M / w H_{\mathrm{M}} w^{-1}\right)$ valued polynomial functions on $\mathfrak{a}_{\mathrm{q}}^{*}$. In particular, if $\lambda \in \mathfrak{a}_{\mathrm{q} \mathfrak{c}}^{*}, D \in \mathbb{D}(G / H)$, then $\mu_{w}(D: \lambda)$ is a differential operator in $\mathbb{D}\left(M / w H_{\mathrm{M}} w^{-1}\right)$; it therefore naturally acts on ${ }^{\circ} \mathcal{C}_{w}(\tau) \simeq C^{\infty}\left(M / w H_{\mathrm{M}} w^{-1}: \tau_{\mathrm{M}}\right)$ by an endomorphism we denote by $\mu_{w}(D: \tau: \lambda)$. Let $\mu(D: \tau: \lambda) \in \operatorname{End}\left({ }^{\circ} \mathcal{C}(\tau)\right)$ be the direct sum of the endomorphisms $\mu_{w}(D: \tau: \lambda), w \in \mathcal{W}$. Then by [13], eqn. (54) we have, for $\psi \in{ }^{\circ} \mathcal{C}(\tau)$ :

$$
D E^{\circ}(\psi: \lambda)=E^{\circ}(\mu(D: \tau: \lambda) \psi: \lambda) \quad(D \in \mathbb{D}(G / H)),
$$

as a meromorphic identity in $\lambda \in \mathfrak{a}_{\mathrm{q}}^{*} \mathbf{c}$. The above equation is also valid with the unnormalized Eisenstein integral $E$ instead of $E^{\circ}$.

The dependence of the Eisenstein integral on the parabolic subgroup $P$ is described by the following functional equation, for $P_{1}, P_{2} \in \mathcal{P}_{\sigma}^{\min }, \psi \in{ }^{\circ} \mathcal{C}(\tau)$ and $s \in W$ (see [13], Prop. 4):

$$
E^{\circ}\left(P_{2}: C_{P_{2} \mid P_{1}}^{\circ}(s: \lambda) \psi: s \lambda\right)=E^{\circ}\left(P_{1}: \psi: \lambda\right)
$$


As suggested by the equations (36) and (37) we have the following relation between the action on ${ }^{\circ} \mathcal{C}(\tau)$ of $\mathbb{D}(G / H)$ and the $C$-functions (which are endomorphisms of ${ }^{\circ} \mathcal{C}(\tau)$ ), for $P, Q \in \mathcal{P}_{\sigma}^{\min }, s \in W$ :

$$
C_{Q \mid P}^{\circ}(s: \lambda) \circ \mu(D: \tau: \lambda)=\mu(D: \tau: s \lambda) \circ C_{Q \mid P}^{\circ}(s: \lambda) \quad(D \in \mathbb{D}(G / H))
$$

as a meromorphic identity in $\lambda \in \mathfrak{a}_{\mathrm{q} \mathfrak{c}}^{*}$, cf. [13], eqn. (73).

The endomorphisms $\mu(D: \tau: \lambda)$ of ${ }^{\circ} \mathcal{C}(\tau)$ admit a simultaneous diagonalization: Let $\mathfrak{b}$ be a Cartan subspace of $\mathfrak{q}$ containing $\mathfrak{a}_{\mathrm{q}}$. Then $\mathfrak{b}=\mathfrak{b}_{\mathrm{k}} \oplus \mathfrak{a}_{\mathrm{q}}$, where $\mathfrak{b}_{\mathrm{k}}:=\mathfrak{b} \cap \mathfrak{k}$. Via this decomposition we identify $\mathfrak{a}_{\mathrm{q} c}^{*}$ and $\mathfrak{b}_{\mathrm{k} c}^{*}$ with subspaces of $\mathfrak{b}_{\mathbf{c}}^{*}$. Let $\gamma: \mathbb{D}(G / H) \rightarrow I(\mathfrak{b}) \subset$ $S(\mathfrak{b})$ be the Harish-Chandra isomorphism. Given $\Lambda \in \mathfrak{b}_{\mathrm{k} \mathfrak{c}}^{*}$ we define the algebra homomorphism $\gamma_{\Lambda}: \mathbb{D}(G / H) \rightarrow S\left(\mathfrak{a}_{\mathrm{q}}\right)$ by $\gamma_{\Lambda}(D)(\lambda)=\gamma(D: \Lambda+\lambda)$, for $D \in \mathbb{D}(G / H)$, and $\lambda \in \mathfrak{a}_{\mathrm{q} c}^{*}$. Correspondingly we have the joint eigenspace

$$
{ }^{\circ} \mathcal{C}(\tau)[\Lambda]:=\bigcap_{\substack{D \in \mathbb{D}(G / H) \\ \lambda \in \mathfrak{a}_{\mathrm{q}}^{*} \mathbf{c}}} \operatorname{ker}\left[\mu(D: \tau: \lambda)-\gamma_{\Lambda}(D)(\lambda)\right] .
$$

Then according to [7], Prop. 4.7, there exists a finite subset $L \subset i \mathfrak{b}_{\mathrm{k}}^{*}$ such that

$$
{ }^{\circ} \mathcal{C}(\tau)=\bigoplus_{\Lambda \in L}{ }^{\circ} \mathcal{C}(\tau)[\Lambda]
$$

the decomposition being orthogonal.

The decomposition into joint eigenspaces becomes simpler if $D$ ranges over $\mathbb{Z}(G / H)=$ $r(\mathfrak{Z})$. Recall from (19) that $\mathfrak{j}$ is a Cartan subalgebra of $\mathfrak{g}$ containing $\mathfrak{a}_{\mathrm{q}}$. Moreover, $\gamma_{\mathfrak{j}}^{\mathfrak{g}}$ is the Harish-Chandra isomorphism from $\mathfrak{Z}$ onto $I(\mathfrak{j})$.

Lemma 5.7 Let $[\xi] \in \widehat{M}_{H}, \lambda \in \mathfrak{a}_{\mathrm{qc}}^{*}$ and $Z \in \mathfrak{Z}$. Then the endomorphism $\mu(r(Z): \tau: \lambda)$ acts by multiplication with the scalar $\gamma_{j}^{\mathfrak{g}}\left(Z: \lambda-\Lambda_{\xi}\right)$ on ${ }^{\circ} \mathcal{C}_{\xi}(\tau)$; here $\Lambda_{\xi} \in \mathfrak{j}_{0 \mathrm{c}}^{*}$ is the infinitesimal character of $\xi$.

Proof. Since the endomorphism depends polynomially on $\lambda$ and linearly on $Z$, we may as well assume that $\lambda \in i \mathfrak{a}_{\mathbf{q}}^{*}$ and that $Z \in \mathfrak{Z}$ is real (with respect to the real form $\mathfrak{g}$ of $\mathfrak{g}_{\mathbf{c}}$ ). Then the representation $\pi_{\xi, \bar{\lambda}}$ has the infinitesimal character $\Lambda_{\xi}+\bar{\lambda}=\Lambda_{\xi}-\lambda$. Moreover, since $\pi_{\xi, \bar{\lambda}}$ is unitary, it follows that

$$
\gamma_{j}^{\mathfrak{g}}\left(Z: \Lambda_{\xi}-\lambda\right)=\overline{\gamma_{j}^{\mathfrak{g}}\left(Z^{\vee}: \Lambda_{\xi}-\lambda\right)}=\overline{\gamma_{j}^{\mathfrak{g}}\left(Z: \lambda-\Lambda_{\xi}\right)}
$$

Combining this with $(20)$ we see that, for every $\eta \in V(\xi)$, we have:

$$
\mu(r(Z): \xi: \bar{\lambda}) \eta=\overline{\gamma_{j}^{\mathfrak{g}}\left(Z: \lambda-\Lambda_{\xi}\right)} \eta
$$

Combining the obtained equation with [13], eqn. (43), and applying Lemma 3 of loc.cit. we see that $\mu(r(Z): \tau: \lambda)$ acts by multiplication with $\gamma_{j}^{\mathfrak{g}}\left(Z: \lambda-\Lambda_{\xi}\right)$ on ${ }^{\circ} \mathcal{C}_{\xi}(\tau)$. 
We end this section by recalling a result that will be used a number of times in the rest of this paper. If $D \in \mathbb{D}(G / H)$, then by $D^{*}$ we denote the formal adjoint of $D$ with respect to the measure $d x$ of $G / H$, i.e. it is defined by the formula $\langle D f \mid g\rangle=\left\langle f \mid D^{*} g\right\rangle$ for all $f, g \in C_{c}^{\infty}(G / H)$, where $\langle\cdot \mid \cdot\rangle$ denotes the $L^{2}$-inner product. For all $D \in \mathbb{D}(G / H), \lambda \in \mathfrak{a}_{\mathrm{q} c}^{*}$ we have $D^{*} \in \mathbb{D}(G / H)$ and:

$$
\mu(D: \tau: \lambda)^{*}=\mu\left(D^{*}: \tau:-\bar{\lambda}\right) .
$$

Here the star on the left hand-side indicates the adjoint with respect to the Hilbert structure of ${ }^{\circ} \mathcal{C}(\tau)$. The relation (40) is an immediate consequence of [13], eqns. (41) and (43).

\section{The $\tau$-spherical Fourier transform}

Let $\tau$ be a unitary representation of $K$ in a finite dimensional Hilbert space $V_{\tau}$. Following [7], §17, we shall define the $\tau$-spherical Fourier transform on a space of $\tau$-spherical Schwartz functions.

Let $\Xi$ be Harish-Chandra's elementary spherical function with spectral parameter zero, associated with the Riemannian symmetric pair $(G, K)$. We define the functions $\Theta, \tau_{\mathrm{G} / \mathrm{H}}: G / H \rightarrow[0, \infty[\mathrm{by}$

$$
\begin{aligned}
\Theta(x H) & =\Xi\left(x \sigma(x)^{-1}\right)^{1 / 2} \quad(x \in G), \\
\tau_{\mathrm{G} / \mathrm{H}}(k a h) & =|\log a| \quad\left(k \in K, a \in A_{\mathbf{q}}, h \in H\right) .
\end{aligned}
$$

Following [7], 117 , we now define the space of $\left(L^{2}\right.$-) Schwartz functions $\mathcal{C}\left(G / H, V_{\tau}\right)$ as the space of $C^{\infty}$ functions $f: G / H \rightarrow V_{\tau}$ such that

$$
\sup _{x \in G / H} \Theta(x)^{-1}\left(1+\tau_{\mathrm{G} / \mathrm{H}}(x)\right)^{n}\left\|L_{u} f(x)\right\|<\infty
$$

for all $n \in \mathbb{N}, u \in U(\mathfrak{g})$; here $L_{u}$ denotes the regular action of $u$ from the left on smooth functions on $G / H$. Equipped with the obvious collection of seminorms this space is a Fréchet space. We denote its closed subspace of $\tau$-spherical functions by $\mathcal{C}(G / H: \tau)$.

If $f, g: G / H \rightarrow V_{\tau}$ are $\tau$-spherical functions such that the function $x \mapsto\langle f(x) \mid g(x)\rangle$ is integrable on $G / H$, then we define

$$
\langle f \mid g\rangle:=\int_{G / H}\langle f(x) \mid g(x)\rangle d x .
$$

Let $P \in \mathcal{P}_{\sigma}^{\text {min }}$ be fixed. It follows from Proposition 5.4 and from the remark succeeding Thm. 19.1 in [7], that for $f \in \mathcal{C}(G / H: \tau)$ the function $x \mapsto\left\langle f(x) \mid E^{\circ}(P: \psi: \lambda)(x)\right\rangle$ is integrable on $G / H$, for every $\psi \in{ }^{\circ} \mathcal{C}(\tau)$. We define the Fourier transform $\mathcal{F} f=\mathcal{F}_{P} f$ of $f$ to be the function $i \mathfrak{a}_{\mathrm{q}}^{*} \rightarrow{ }^{\circ} \mathcal{C}(\tau)$ determined by:

$$
\langle\mathcal{F} f(\lambda) \mid \psi\rangle=\left\langle f \mid E^{\circ}(P: \psi:-\bar{\lambda})\right\rangle,
$$

for all $\psi \in{ }^{\circ} \mathcal{C}(\tau)$. Let $\mathcal{S}\left(i \mathfrak{a}_{\mathrm{q}}^{*}\right)$ denote the (Schwartz) space of rapidly decreasing functions on the Euclidean space $i \mathfrak{a}_{\mathrm{q}}^{*}$, then we now have (cf. [13], Cor. 4 ): 
Lemma 6.1 The Fourier transform $\mathcal{F}$ is a continuous linear map from $\mathcal{C}(G / H: \tau)$ to $\mathcal{S}\left(i \mathfrak{a}_{\mathrm{q}}^{*}\right) \otimes{ }^{\circ} \mathcal{C}(\tau)$.

If $f$ is compactly supported then from equation (42) one sees that $\mathcal{F} f$ extends to a meromorphic ${ }^{\circ} \mathcal{C}(\tau)$-valued function on $\mathfrak{a}_{\mathrm{q} c}^{*}$, given by the same equation.

We recall some more properties of the $\tau$-spherical Fourier transform. From [4], Lemma 7.2, it follows that $\mathbb{D}(G / H)$ maps $\mathcal{C}(G / H: \tau)$ continuously into itself. From equations (36), (42) and (40) we obtain the following result (see also [7], Lemma 19.3).

Lemma 6.2 Let $f \in \mathcal{C}(G / H: \tau), D \in \mathbb{D}(G / H)$. Then

$$
\mathcal{F}(D f)(\lambda)=\mu(D: \tau: \lambda) \mathcal{F} f(\lambda) \quad\left(\lambda \in i \mathfrak{a}_{\mathrm{q}}^{*}\right)
$$

The following result is a consequence of (37) combined with Proposition 5.3 (see also [13], eqn. (76)).

Lemma 6.3 Let $P_{1}, P_{2} \in \mathcal{P}_{\sigma}^{\min }$, and $s \in W$. Then for all $f \in \mathcal{C}(G / H: \tau)$ we have:

$$
\mathcal{F}_{P_{2}} f(s \lambda)=C_{P_{2} \mid P_{1}}^{\circ}(s: \lambda) \mathcal{F}_{P_{1}} f(\lambda) \quad\left(\lambda \in i \mathfrak{a}_{\mathrm{q}}^{*}\right) .
$$

We shall now describe the relation of the spherical Fourier transform with the Fourier transform defined in Section 4. Let $\vartheta \subset \widehat{K}$ be a finite subset, and in the rest of this section let $\tau=\tau_{\vartheta}$ be as in Remark 5.1. We denote the spaces of left $K$-finite functions in $L^{2}(G / H)$ and $C_{c}^{\infty}(G / H)$ all of whose $K$-types belong to $\vartheta$ by $L^{2}(G / H)_{\vartheta}$ and $C_{c}^{\infty}(G / H)_{\vartheta}$ respectively. These spaces are closed subspaces of $L^{2}(G / H)$ and $C_{c}^{\infty}(G / H)$, respectively. We equip them with the induced structures, respectively of a Hilbert space and a topological linear space.

The $\operatorname{map} \delta_{e}: \varphi \mapsto \varphi(e)$ defines a linear functional on $V_{\tau}=\mathbf{V}_{\vartheta}$. Moreover, one readily verifies that the map $F \mapsto \delta_{e} \circ F$ is a bijective isometry from $L^{2}(G / H: \tau)$ onto $L^{2}(G / H)_{\vartheta}$. Its inverse,

$$
\varsigma: L^{2}(G / H)_{\vartheta} \stackrel{\simeq}{\longrightarrow} L^{2}(G / H: \tau),
$$

is given by $\varsigma(\varphi)(x)(k)=\varphi(k x)$ for $k \in K, x \in G / H$ (see [13], Lemma 5). Of course, $\varsigma$ maps the space $C_{c}^{\infty}(G / H)_{\vartheta}$ bijectively and continuously onto $C_{c}^{\infty}(G / H: \tau)$, and it intertwines the actions of $\mathbb{D}(G / H)$ on these spaces.

If $f \in C_{c}^{\infty}(G / H)_{\vartheta}$, then it follows from Lemma 4.3 that

$$
\hat{f}(\xi: \lambda) \in C^{\infty}(K: \xi)_{\vartheta} \otimes V(\xi)^{*} \simeq \mathcal{H}_{\xi, \vartheta} \otimes \overline{V(\xi)}
$$

for $[\xi] \in \widehat{M}_{H}, \lambda \in i \mathfrak{a}_{\mathrm{q}}^{*}$.

We now recall the following relation between the $\tau_{\vartheta}$-spherical Fourier transform and the Fourier transform. 
Proposition 6.4 Let $\vartheta \subset \widehat{K}$ be a finite subset, and put $\tau=\tau_{\vartheta}$. Furthermore, let $f \in C_{c}^{\infty}(G / H)_{\vartheta}$ and put $F=\varsigma(f)$. Then for $[\xi] \in \widehat{M}_{H}, T \in \mathcal{H}_{\xi, \vartheta} \otimes \overline{V(\xi)}$ and $\lambda \in i \mathfrak{a}_{\mathrm{q}}^{*}$ we have that

$$
\left\langle\mathcal{F} F(\lambda) \mid \psi_{T}\right\rangle=\langle\hat{f}(\xi: \lambda) \mid T\rangle .
$$

Moreover, for $\lambda \in i \mathfrak{a}_{\mathrm{q}}^{*}$ we have the finite sum

$$
\mathcal{F} F(\lambda)=\sum_{\substack{[\xi] \in \widehat{M}_{H} \\[\xi] \uparrow \tau}} d_{\xi} \psi_{\hat{f}(\xi: \lambda)} .
$$

Proof. The relation (45) is given in [13], Prop. 3, and (46) is equivalent to it as a consequence of (27) and Lemma 5.6.

\section{Expansions of the Eisenstein integral}

In this section $\tau$ will be a fixed unitary representation of $K$ in a finite dimensional Hilbert space $V_{\tau}$, and $P, Q$ will be fixed parabolic subgroups from $\mathcal{P}_{\sigma}^{\min }$. Being a $\mathbb{D}(G / H)$-finite $\tau$-spherical function, the Eisenstein integral $E^{\circ}(\psi: \lambda)=E^{\circ}(P: \psi: \lambda)$ (with $\psi \in{ }^{\circ} \mathcal{C}(\tau)$ ) has a convergent expansion on the chamber $A_{q}^{+}(Q)$ (see [4]). We will recall some specific properties of this expansion, which are obtained in [14] in generalization of Gangolli's paper [21].

We start by recalling from [4] the notion of the $\tau$-radial component of a differential operator from $\mathbb{D}(G / H)$, meanwhile fixing notations that will be used later in this paper.

From the Cartan decomposition (see (5) and the succeeding text) it follows that $K A_{\mathrm{q}}^{+}(Q) H$ is an open subset of $G$. If $f \in C^{\infty}(G / H: \tau)$, then $f$ restricts to a smooth

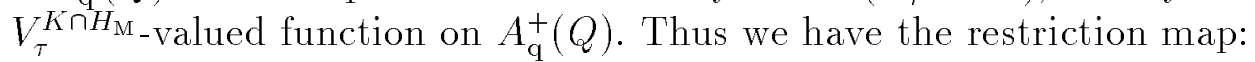

$$
T_{Q}^{\downarrow}: \quad f \mapsto f \mid A_{\mathrm{q}}^{+}(Q), \quad C^{\infty}(G / H: \tau) \rightarrow C^{\infty}\left(A_{\mathrm{q}}^{+}(Q), V_{\tau}^{K \cap H_{\mathrm{M}}}\right),
$$

which is a continuous linear map for the usual locally convex topologies on these spaces.

Let $C_{c}^{\infty}\left(K A_{q}^{+}(Q) H, V_{\tau}\right)$ denote the space of smooth compactly supported $V_{\tau}$-valued functions equipped with the usual complete locally convex topology. The subspace of functions $f$ transforming according to the rule $f(k x h)=\tau(k) f(x)$ for $k \in K, h \in H, x \in$ $K A_{\mathrm{q}}^{+}(Q) H$ is closed; we denote it by $C_{c}^{\infty}\left(K A_{\mathrm{q}}^{+}(Q) H: \tau\right)$ and equip it with the restriction topology. A function of $C_{c}^{\infty}\left(K A_{\mathrm{q}}^{+}(Q) H, V_{\tau}\right)$ may be viewed as a function of $C_{c}^{\infty}\left(G, V_{\tau}\right)$ via extension by zero outside its support. Accordingly we have a continuous linear embedding

$$
C_{c}^{\infty}\left(K A_{\mathrm{q}}^{+}(Q) H: \tau\right) \hookrightarrow C^{\infty}(G / H: \tau)
$$

Via this embedding we identify the linear space on the left-hand side with its image in the space on the right-hand side. This image equals the linear subspace of functions $f \in C^{\infty}(G / H: \tau)$ with supp $f \subset K A_{\mathrm{q}}^{+}(Q) H$. Notice however that the image is not closed, 
hence not complete, as a subspace of $C^{\infty}(G / H: \tau)$; therefore the embedding (48) cannot be used to identify $C_{c}^{\infty}\left(K A_{\mathrm{q}}^{+}(Q) H: \tau\right)$ topologically with a subspace of $C^{\infty}(G / H: \tau)$.

Now recall that the map $(k, h, a) \mapsto k a h$ induces a diffeomorphism from $K \times_{M \cap K \cap H}$ $H \times A_{\mathrm{q}}^{+}(Q)$ onto $K A_{\mathrm{q}}^{+}(Q) H$. From this the following lemma is immediate.

Lemma 7.1 The map $T_{Q}^{\downarrow}$ restricts to a topological linear isomorphism from the space $C_{c}^{\infty}\left(K A_{\mathrm{q}}^{+}(Q) H: \tau\right)$ onto $C_{c}^{\infty}\left(A_{\mathrm{q}}^{+}(Q), V_{\tau}^{K \cap H_{\mathrm{M}}}\right)$.

We define the continuous linear map

$$
T_{Q}^{\dagger}: C_{c}^{\infty}\left(A_{q}^{+}(Q), V_{\tau}^{K \cap H_{\mathrm{M}}}\right) \rightarrow C^{\infty}(G / H: \tau)
$$

as the inverse of the topological linear isomorphism of the above lemma, composed with the embedding (48). For later purposes we list:

$$
\begin{array}{llll}
T_{Q}^{\downarrow} \circ T_{Q}^{\dagger} & =I & \text { on } & C_{c}^{\infty}\left(A_{q}^{+}(Q), V_{\tau}^{K \cap H_{\mathrm{M}}}\right) ; \\
T_{Q}^{\dagger} \circ T_{Q}^{\downarrow}=I & \text { on } & C_{c}^{\infty}\left(K A_{\mathrm{q}}^{+}(Q) H: \tau\right) .
\end{array}
$$

Notice that if for $g, h \in C_{c}^{\infty}\left(A_{\mathrm{q}}^{+}(Q), V_{\tau}^{K \cap H_{\mathrm{M}}}\right)$ we define

$$
\langle g \mid h\rangle_{J}:=\int_{A_{\mathrm{q}}^{+}(Q)}\langle g(a) \mid h(a)\rangle_{\tau} J(a) d a,
$$

then from (21) (with $C=1$ ) we see that $T_{Q}^{\dagger}$ is the transposed of $T_{Q}^{\downarrow}$ in the sense that

$$
\left\langle f \mid T_{Q}^{\dagger} g\right\rangle=\left\langle T_{Q}^{\downarrow} f \mid g\right\rangle_{J}
$$

for all $f \in C_{c}^{\infty}\left(K A_{\mathrm{q}}^{+}(Q) H: \tau\right)$ and $g \in C_{c}^{\infty}\left(A_{\mathrm{q}}^{+}(Q), V_{\tau}^{K \cap H_{\mathrm{M}}}\right)$.

If $D \in \mathbb{D}(G / H)$ then one readily checks that

$$
\Pi_{Q, \tau}(D):=T_{Q}^{\downarrow} \circ D \circ T_{Q}^{\dagger}
$$

defines an element of the ring

$$
C^{\infty}\left(A_{\mathrm{q}}^{+}(Q)\right) \otimes S\left(\mathfrak{a}_{\mathrm{q}}\right) \otimes \operatorname{End}\left(V_{\tau}^{K \cap H_{\mathrm{M}}}\right)
$$

of differential operators on $A_{\mathrm{q}}^{+}(Q)$, with coefficients in $C^{\infty}\left(A_{\mathrm{q}}^{+}(Q)\right) \otimes \operatorname{End}\left(V_{\tau}^{K \cap H_{\mathrm{M}}}\right)$. The operator (54) is called the $\tau$-radial component of $D$ on $A_{\mathrm{q}}^{+}(Q)$. Using (51) one readily sees that $\Pi_{Q, \tau}$ is an algebra homomorphism from $\mathbb{D}(G / H)$ to $(55)$. Note that if a function $f \in C^{\infty}(G / H: \tau)$ is $\mathbb{D}(G / H)$-finite, then its restriction to $A_{\mathrm{q}}^{+}(Q)$ is finite under the algebra $\Pi_{Q, \tau}(\mathbb{D}(G / H))$.

If $X \in U(\mathfrak{g})^{H}$, then we shall also write $\Pi_{Q, \tau}(X)$ for $\Pi_{Q, \tau}(r(X))($ cf. (12)). Thus we also view $\Pi_{Q, \tau}$ as an algebra homomorphism from $U(\mathfrak{g})^{H}$ to $(55)$.

Let $\mathcal{O}=\mathcal{O}_{Q}$ denote the ring of functions $\Phi: A_{\mathrm{q}}^{+}(Q) \rightarrow \mathbb{C}$ which admit a converging series expansion of the form

$$
\Phi(a)=\sum_{\nu \in \mathbb{N} \Sigma(Q)} c_{\nu} a^{-\nu} \quad\left(a \in A_{\mathrm{q}}^{+}(Q)\right)
$$

with coefficients $c_{\nu} \in \mathbb{C}$. 
Remark 7.2 Let $\Delta(Q)$ be the set of simple roots in $\Sigma(Q)$. If $\nu \in \mathbb{N} \Sigma(Q)$, we write $\nu=\sum_{\alpha \in \Delta(Q)} \nu_{\alpha} \alpha$. If moreover $z \in \mathbb{C}^{\Delta(Q)}$, we write

$$
z^{\nu}=\prod_{\alpha \in \Delta(Q)} z_{\alpha}^{\nu_{\alpha}}
$$

Let $D$ denote the unit disk in $\mathbb{C}$. Then obviously the series (56) defines a function in $\mathcal{O}$ if and only if the power series $\sum_{\nu \in \mathbb{N} \Sigma(Q)} c_{\nu} z^{\nu}$ converges on the polydisk $D^{\Delta(Q)}$ (and hence defines a holomorphic function $\varphi$ on that set). This explains the use of the notation $\mathcal{O}$.

Let $\mathbf{z}=\mathbf{z}_{Q}: A_{\mathbf{q}}^{+}(Q) \rightarrow D^{\Delta(Q)}$ be the map defined by $\mathbf{z}_{\alpha}(a)=a^{-\alpha}(\alpha \in \Delta(Q))$. Then the above functions $\Phi$ and $\varphi$ correspond to each other by $\Phi(a)=\varphi(\mathbf{z}(a))$. Operations like term by term differentiation which can be applied to power series, can therefore be applied to elements of $\mathcal{O}$ as well.

The following result is now a straightforward consequence of [4], Prop. 3.5, Lemma 3.6 and Prop. 3.7.

Lemma 7.3 The map $\Pi_{Q, \tau}$ is an algebra homomorphism from $U(\mathfrak{g})^{H}$ to $\mathcal{O}_{Q} \otimes S\left(\mathfrak{a}_{\mathbf{q}}\right) \otimes$ $\operatorname{End}\left(V_{\tau}^{K \cap H_{\mathrm{M}}}\right)$.

In the rest of this section we shall write $\Sigma^{+}=\Sigma(Q)$, and we shall suppress the dependence on $Q$ in the notations. In particular we write $A_{\mathrm{q}}^{+}=A_{\mathrm{q}}^{+}(Q), \Delta=\Delta(Q)$, $\rho=\rho_{Q}$, and $\Pi_{\tau}=\Pi_{Q, \tau}$.

If $\nu=\sum_{\alpha \in \Delta} \nu_{\alpha} \alpha \in \mathbb{N} \Delta$, we write $m(\nu)=\sum_{\alpha}\left|\nu_{\alpha}\right|$. Let $\tau\left(\Omega_{\mathfrak{m}}\right)$ denote the endomorphism by which the ' $\mathfrak{m} \cap \mathfrak{k}$-part' of the Casimir element of $U(\mathfrak{g})$ acts on $V_{\tau}^{K \cap H_{\mathrm{M}}}$ (see [14] for details), and let $\gamma \in \operatorname{End}\left(\operatorname{End}\left(V_{\tau}^{K \cap H_{\mathrm{M}}}\right)\right)$ be defined by $\gamma=\operatorname{ad}\left(\tau\left(\Omega_{\mathfrak{m}}\right)\right)$. Then the set $\mathcal{N}$ of eigenvalues of $\gamma$ is a symmetric subset of $\mathbb{R}$. Put

$$
\mathrm{S}=\left\{\lambda \in \mathfrak{a}_{\mathrm{q}}^{*} \mid \exists(\nu \in \mathbb{N} \Delta \backslash\{0\}):\langle 2 \lambda-\nu, \nu\rangle \in \mathcal{N}\right\}
$$

One readily checks that the evaluation map $f \mapsto f(e)$ defines a linear isometry

$$
\mathrm{ev}_{1}:{ }^{\circ} \mathcal{C}_{1}(\tau) \simeq C^{\infty}\left(M / H_{\mathrm{M}}: \tau_{\mathrm{M}}\right) \stackrel{\simeq}{\longrightarrow} V_{\tau}^{K \cap H_{\mathrm{M}}}
$$

Via this isomorphism we endow $V_{\tau}^{K \cap H_{\mathrm{M}}}$ with the structure of a $\mathbb{D}\left(M / H_{\mathrm{M}}\right)$-module. Recall the definition of the algebra homomorphism $\mu: \mathbb{D}(G / H) \rightarrow \mathbb{D}\left(M_{1} / H_{\mathrm{M}_{1}}\right) \simeq \mathbb{D}\left(M / H_{\mathrm{M}}\right) \otimes$ $S\left(\mathfrak{a}_{\mathrm{q}}\right)$ from Section 2. If $D \in \mathbb{D}(G / H), \lambda \in \mathfrak{a}_{\mathrm{q}}^{*}$, we denote the endomorphism by which the operator $\mu(D: \lambda)$ acts on $V_{\tau}^{K \cap H_{\mathrm{M}}}$ by $\underline{\mu}(D: \tau: \lambda)$. Notice that this endomorphism is related to the endomorphism $\mu_{1}(D: \tau: \lambda)$ on ${ }^{\circ} \mathcal{C}_{1}(\tau)$ defined above $(36)$ by

$$
\mathrm{ev}_{1} \circ \mu_{1}(D: \tau: \lambda)=\underline{\mu}(D: \tau: \lambda) \circ \mathrm{ev}_{1} .
$$

Let $\mathrm{L} \in \mathbb{D}(G / H)$ denote the image of the Casimir element of $U(\mathfrak{g})$ under the map $r$ defined in (12). ( $\mathrm{L}$ is the Laplace-Beltrami operator associated with the natural pseudoRiemannian structure on $G / H$ induced by the bilinear form $B$.) 
Proposition 7.4 ([14]) Let $\lambda \in \mathfrak{a}_{\mathrm{q}}^{*} \backslash \mathrm{S}$, where $\mathrm{S}$ is defined by (57). Then there exists a unique function $\Phi_{\lambda}=\Phi_{Q, \lambda} \in a^{\lambda-\rho} \mathcal{O} \otimes \operatorname{End}\left(V_{\tau}^{K \cap H_{\mathrm{M}}}\right)$ having an expansion of the form

$$
\Phi_{\lambda}(a)=a^{\lambda-\rho} \sum_{\nu \in \mathbb{N} \Delta},{ }_{\mu}(\lambda) a^{-\nu} \quad\left(a \in A_{\mathrm{q}}^{+}\right)
$$

with $,{ }_{\nu}(\lambda) \in \operatorname{End}\left(V_{\tau}^{K \cap H_{\mathrm{M}}}\right),{ }_{0}(\lambda)=I$, such that:

$$
\left[\Pi_{\tau}(\mathrm{L}) \Phi_{\lambda}\right](a)=\Phi_{\lambda}(a) \circ \underline{\mu}(\mathrm{L}: \tau: \lambda) \quad\left(a \in A_{\mathrm{q}}^{+}\right) .
$$

Finally, $\Phi_{\lambda}(a)$ and the ${ }_{\nu}(\lambda)\left(a \in A_{\mathrm{q}}^{+}, \nu \in \mathbb{N} \Delta\right)$ extend to meromorphic functions of $\lambda \in \mathfrak{a}_{\mathrm{q}}^{*}$ with singular set contained in $\mathrm{S}$.

The functions $\Phi_{\lambda}$ occur in the expansion of the normalized Eisenstein integral as follows. If $w \in \mathcal{W}, \lambda \in \mathfrak{a}_{\mathrm{qc}}^{*} \backslash S$ we define the $\operatorname{End}\left(V_{\tau}^{K \cap w H_{\mathrm{M}} w^{-1}}\right)$-valued function $\Phi_{Q, w}(\lambda: \cdot)$ on $A_{\mathrm{q}}^{+}(Q)$ by

$$
\Phi_{Q, w}(\lambda: a)=\tau(w) \circ \Phi_{Q, \lambda}(a) \circ \tau(w)^{-1} .
$$

Then we have the following.

Theorem 7.5 ([14]) Let $P, Q \in \mathcal{P}_{\sigma}^{\min }, w \in \mathcal{W}$. Then for every $\psi \in{ }^{\circ} \mathcal{C}(\tau)$ we have

$$
E^{\circ}(P: \psi: \lambda)(a w)=\sum_{s \in W} \Phi_{Q, w}(s \lambda: a)\left[\operatorname{pr}_{w} C_{Q \mid P}^{\circ}(s: \lambda) \psi\right](e) \quad\left(a \in A_{\mathrm{q}}^{+}(Q)\right)
$$

as a meromorphic identity in $\lambda$.

Note that the above identity remains valid if $E^{\circ}(\psi: \lambda)$ and $C_{Q \mid P}^{\circ}(s: \lambda)$ are replaced by their unnormalized versions $E(\psi: \lambda)$ and $C_{Q \mid P}(s: \lambda)$ respectively.

Let $\left.J: A_{\mathrm{q}}^{+} \rightarrow\right] 0, \infty\left[\right.$ be the Jacobian introduced in Section 3 . Then for $\lambda \in \mathfrak{a}_{\mathrm{qc}}^{*} \backslash \mathrm{S}$ we define the function $\tilde{\Phi}_{\lambda}: A_{\mathrm{q}}^{+} \rightarrow \operatorname{End}\left(V_{\tau}^{K \cap H_{\mathrm{M}}}\right)$ by

$$
\tilde{\Phi}_{\lambda}(a)=J(a)^{1 / 2} \Phi_{\lambda}(a) \quad\left(a \in A_{\mathrm{q}}^{+}\right) .
$$

Now obviously $a^{-\rho} J(a)^{1 / 2} \in \mathcal{O}$, and therefore the function $\tilde{\Phi}_{\lambda}$ has an expansion of the form

$$
\tilde{\Phi}_{\lambda}(a)=a^{\lambda} \sum_{\nu \in \mathbb{N} \Delta} a^{-\nu}{ }^{\sim}{ }_{\nu}(\lambda)
$$

with coefficients ${ }^{\sim}{ }_{\nu}(\lambda) \in \operatorname{End}\left(V_{\tau}^{K \cap H_{\mathrm{M}}}\right), \nu \in \mathbb{N} \Delta$.

If $R \in \mathbb{R}$, we define the following finite subset of $\mathbb{N} \Delta$ :

$$
X_{R}=\left\{\nu \in \mathbb{N} \Delta \backslash\{0\} \mid\|\nu\|^{2}-2 \operatorname{Rm}(\nu)+\min \mathcal{N} \leq 0\right\} .
$$

Moreover, we define the following polynomial function of $\lambda$ :

$$
p_{R}(\lambda)=\prod_{\nu \in X_{R}} \prod_{d \in \mathcal{N}}(\langle 2 \lambda-\nu, \nu\rangle-d) .
$$

(If $X_{R}$ is empty then by definition $p_{R}(\lambda)=1$.)

The following estimates will play a crucial role in Sections 10 and 13 . Let $\overline{\mathfrak{a}}_{\mathrm{q}}^{*}(Q, R)$ denote the closure of $\mathfrak{a}_{\mathrm{q}}^{*}(Q, R)$ in $\mathfrak{a}_{\mathrm{q}}^{*}$. 
Theorem 7.6 ([14]) Let $R \in \mathbb{R}$. Then there exist constants $C>0, \kappa>0$ (depending on $\tau, R$ ) such that

$$
\left|p_{R}(\lambda)\right|\left\|^{\sim}{ }_{\nu}(\lambda)\right\|_{\text {op }} \leq C(1+|\nu|)^{\kappa}(1+|\lambda|)^{\operatorname{deg} p_{R}},
$$

for all $\nu \in \mathbb{N} \Delta$ and $\lambda \in \overline{\mathfrak{a}}_{\mathrm{q}}^{*}(Q, R)$. Here $\|\cdot\|_{\text {op }}$ denotes the operator norm.

Corollary 7.7 ([14]) Let $R \in \mathbb{R}$. Then we have the following:

(a) if $a \in A_{\mathrm{q}}^{+}$, then the function $\lambda \mapsto p_{R}(\lambda) \Phi_{\lambda}(a)$ is holomorphic in a neighborhood of $\overline{\mathfrak{a}}_{\mathrm{q}}^{\star}(Q, R)$;

(b) there exists for each $\epsilon>0$ a constant $C>0$ (depending on $\tau, R$ ) such that

$$
\left|p_{R}(\lambda)\right|\left\|\Phi_{\lambda}(a)\right\|_{\text {op }} \leq C a^{\operatorname{Re} \lambda-\rho}(1+|\lambda|)^{\operatorname{deg} p_{R}}
$$

for all $\lambda \in \overline{\mathfrak{a}}_{\mathrm{q}}^{*}(Q, R)$, and all $a \in A_{\mathrm{q}}$ with $\alpha(\log a)>\epsilon\left(\forall \alpha \in \Sigma^{+}\right)$.

Remark 7.8 Notice that if $G / H$ is split, that is, if $\mathfrak{a}_{\mathrm{q}}$ is also maximal abelian in $\mathfrak{q}$, then $\mathfrak{m} \subset \mathfrak{h}$ so that $\tau\left(\Omega_{\mathfrak{m}}\right)$ acts trivially on $V_{\tau}^{K \cap H_{\mathrm{M}}}$. Hence $\mathcal{N}=\{0\}$, from which we infer that if $R \leq 0$ then $X_{R}$ is empty and $p_{R}=1$. In particular this is the case when $G / H$ is a Riemannian symmetric space. The same conclusion holds for the trivial $K$-type $\tau$ on an arbitrary reductive symmetric space $G / H$.

\section{Paley-Wiener estimates for the Fourier transform}

Let $\left(\tau, V_{\tau}\right)$ be a finite dimensional unitary representation of $K$, and let $P \in \mathcal{P}_{\sigma}^{\min }$. If $f \in C_{c}^{\infty}(G / H: \tau)$ then its $\tau$-spherical Fourier transform $\mathcal{F} f=\mathcal{F}_{P} f$, originally defined as a ${ }^{\circ} \mathcal{C}(\tau)$-valued Schwartz function on $i \mathfrak{a}_{\mathrm{q}}^{*}$, has a unique meromorphic extension to all of $\mathfrak{a}_{\mathrm{q} c}^{*}$. Thus it may be viewed as an element of the space $\mathcal{M} \otimes{ }^{\circ} \mathcal{C}(\tau)$ of meromorphic ${ }^{\circ} \mathcal{C}(\tau)$-valued functions on $\mathfrak{a}_{\mathrm{q}}^{*}$. The purpose of this section is to give estimates of Paley-Wiener type, relating the growth of $\mathcal{F} f$ to the support of $f$.

We start with a preparatory lemma. The set $\Pi_{\Sigma}\left(\mathfrak{a}_{\mathrm{q}}\right)$, defined in Section 5 , is partially ordered by the relation of division. Let ' $\pi \in \Pi_{\Sigma}\left(\mathfrak{a}_{\mathrm{q}}\right)$ be a polynomial which is minimal subject to the condition that for every $\psi \in{ }^{\circ} \mathcal{C}(\tau)$ the function $E_{\mathbf{i}_{\pi}}: \lambda \mapsto{ }^{\prime} \pi(\lambda) E^{\circ}(P: \psi: \lambda)$ is holomorphic in $\mathfrak{a}_{\mathrm{q}}^{*}(\bar{P}, 0)$; for its existence, $\mathrm{cf}$. (34). The polynomial ' $\pi$ is uniquely determined up to a non-zero scalar factor.

Lemma 8.1 There exists a constant $\epsilon>0$ such that:

(a) The polynomial ' $\pi$ has no zeros in $\mathfrak{a}_{\mathrm{q}}^{*}(\epsilon)$;

(b) For every $\psi \in{ }^{\circ} \mathcal{C}(\tau)$ the function $E_{\mathbf{i}_{\pi}}^{\circ}: \lambda \mapsto{ }^{\prime} \pi(\lambda) E^{\circ}(P: \psi: \lambda)$ is holomorphic in $\mathfrak{a}_{\mathrm{q}}^{*}(\bar{P}, \epsilon)$. 
Proof. If $l: \lambda \mapsto\langle\lambda, \alpha\rangle-c$ is a linear factor of ' $\pi(\alpha \in \Sigma(\bar{P}), c \in \mathbb{C})$, then by minimality of ' $\pi$ we must have $l^{-1}(0) \cap \mathfrak{a}_{\mathrm{q}}^{*}(\bar{P}, 0) \neq \emptyset$. Hence $\operatorname{Re} c<0$, and we see that there exists a constant $\epsilon>0$ such that $l^{-1}(0) \cap \mathfrak{a}_{\mathrm{q}}^{*}(\epsilon)=\emptyset$. Since ' $\pi$ is a product of linear factors like the above, there exists a constant $\epsilon>0$ such that (a) holds.

The function $E_{\mathfrak{i}_{\pi}}$ is regular on the set $i \mathfrak{a}_{\mathrm{q}}^{*}$. Moreover, by (34) there exists a polynomial $p \in \Pi_{\Sigma}\left(\mathfrak{a}_{\mathrm{q}}\right)$ such that $p E_{\mathfrak{i}_{\pi}}$ is regular on $\mathfrak{a}_{\mathrm{q}}^{*}(\bar{P}, 1)$ (for any $\psi$ ). Applying the lemma below with $\eta_{1}=1$ and $\varphi=E_{i_{\pi}}$, we see that there exists $\eta_{2}>0$, independent of $\psi$, such that $E_{\mathfrak{i}_{\pi}}$ is regular on the set $\mathfrak{a}_{\mathrm{q}}^{*}\left(\bar{P}, \eta_{2}\right)$. Now decrease the $\epsilon$ found in the first part of the proof so that $\epsilon \leq \eta_{2}$.

Lemma 8.2 Let $\eta_{1}>0$ and $p \in \Pi_{\Sigma}\left(\mathfrak{a}_{q}\right)$. Then there exists a constant $\eta_{2}>0$ such that the following holds. Suppose $\varphi: \mathfrak{a}_{\mathrm{q}}^{*}\left(\bar{P}, \eta_{1}\right) \rightarrow \mathbb{C}$ is a meromorphic function such that:

(a) $p \varphi$ is regular on $\mathfrak{a}_{\mathrm{q}}^{*}\left(\bar{P}, \eta_{1}\right)$;

(b) $\varphi$ is regular on $\mathfrak{a}_{\mathrm{q}}^{*}(\bar{P}, 0) \cup i \mathfrak{a}_{\mathrm{q}}^{*}$.

Then the function $\varphi$ is regular on $\mathfrak{a}_{\mathrm{q}}^{*}\left(\bar{P}, \eta_{2}\right)$.

Proof. We may restrict ourselves to the case that $p$ is a first degree polynomial of the form $\lambda \mapsto\langle\lambda, \alpha\rangle-c, \alpha \in \Sigma(\bar{P}), c \in \mathbb{C}$, since (up to a sign) every polynomial of $\Pi_{\Sigma}\left(\mathfrak{a}_{\mathrm{q}}\right)$ is a product of such first degree polynomials. We now distinguish the following three cases: (i) $\operatorname{Re} c<0$; (ii) $\operatorname{Re} c=0$; (iii) $\operatorname{Re} c>0$.

(i) In this case $U_{1}:=p^{-1}(0) \cap \mathfrak{a}_{\mathrm{q}}^{*}(\bar{P}, 0)$ is a non-empty open subset of the complex hyperplane $p^{-1}(0)$. By (b) it follows that $p \varphi$ vanishes on $U_{1}$; the latter set is contained in the convex, hence connected, open subset $U_{1}^{\prime}:=p^{-1}(0) \cap \mathfrak{a}_{\mathrm{q}}^{*}\left(\bar{P}, \eta_{1}\right)$ of $p^{-1}(0)$. By analytic continuation it follows that $p \varphi$ vanishes on $U_{1}^{\prime}$. Hence on the set $\mathfrak{a}_{\mathrm{q}}^{*}\left(\bar{P}, \eta_{1}\right)$ the first degree polynomial $p$ is a divisor of the holomorphic function $p \varphi$, and the conclusion follows with $\eta_{2}=\eta_{1}$.

(ii) In this case $U_{2}:=p^{-1}(0) \cap i \mathfrak{a}_{\mathrm{q}}^{*}$ is a non-empty real subspace of the complex hyperplane $p^{-1}(0)$. By (b) the function $p \varphi$ vanishes on $U_{2}$. We may now apply analytic continuation as in (i) and deduce the conclusion with $\eta_{2}=\eta_{1}$.

(iii) In this case we put $\eta_{2}=\min \left(\eta_{1}, \operatorname{Re} c\right)$. Then $p$ has no zeros on $\mathfrak{a}_{\mathrm{q}}^{*}\left(\bar{P}, \eta_{2}\right)$, and $p \varphi$ is holomorphic on $\mathfrak{a}_{\mathrm{q}}^{*}\left(\bar{P}, \eta_{2}\right) \subset \mathfrak{a}_{\mathrm{q}}^{*}\left(\bar{P}, \eta_{1}\right)$, by assumption (a). From this the desired conclusion follows.

Define the polynomial $\pi \in \Pi_{\Sigma}\left(\mathfrak{a}_{\mathrm{q}}\right)$ by

$$
\pi(\lambda)=\overline{{ }^{\prime} \pi(-\bar{\lambda})}
$$

Let $\epsilon>0$ be as in Lemma 8.1. Then from (42) it follows that for every $f \in C_{c}^{\infty}(G / H: \tau)$ the function $\lambda \mapsto \pi(\lambda) \mathcal{F} f(\lambda)$ is holomorphic on $-\mathfrak{a}_{\mathrm{q}}^{*}(\bar{P}, \epsilon)=\mathfrak{a}_{\mathrm{q}}^{*}(P, \epsilon)$.

By [7], Cor. 16.2 and Prop. 10.3 we may shrink $\epsilon$ to ensure the existence of constants $C>0, N \in \mathbb{N}, s>0$ such that:

$$
\left\|{ }^{\prime} \pi(\lambda) E^{\circ}(P: \psi: \lambda)(a)\right\| \leq C(1+|\lambda|)^{N} e^{(s+|\operatorname{Re} \lambda|)|\log a|}\|\psi\|,
$$


for all $\psi \in{ }^{\circ} \mathcal{C}(\tau), a \in A_{\mathrm{q}}$ and $\lambda \in \mathfrak{a}_{\mathrm{q}}^{*}(\bar{P}, \epsilon)$. We shall use this estimate to obtain a crude estimate for $\mathcal{F} f$. Let $S$ be a subset of $\mathfrak{a}_{\mathrm{q}}$ and define the subset $X_{S}$ of $G / H$ by

$$
X_{S}=K \exp S H
$$

If $S$ is compact then so is $X_{S}$, and we equip the space

$$
C_{S}^{\infty}(G / H: \tau):=\left\{f \in C_{c}^{\infty}(G / H: \tau) \mid \operatorname{supp} f \subset X_{S}\right\}
$$

with the usual Fréchet topology. Put

$$
R_{S}=\sup _{Y \in S}|Y|
$$

(If $S=\emptyset$ then $R_{S}=-\infty$.) Then we have the following:

Proposition 8.3 Let $\epsilon>0$ be as above. Then for every $n \in \mathbb{N}$ there exists a continuous seminorm $\nu$ on $C_{S}^{\infty}(G / H: \tau)$ such that for all $f \in C_{S}^{\infty}(G / H: \tau)$ and every $\lambda \in \mathfrak{a}_{\mathrm{q}}^{*}(P, \epsilon)$ we have:

$$
\|\pi(\lambda) \mathcal{F} f(\lambda)\| \leq(1+|\lambda|)^{-n} e^{R_{S}|\operatorname{Re} \lambda|} \nu(f) .
$$

Proof. From the above estimate for the Eisenstein integral it follows straightforwardly that there exist constants $C^{\prime}$ and $N$ such that

$$
\|\pi(\lambda) \mathcal{F} f(\lambda)\| \leq C^{\prime}(1+|\lambda|)^{N} e^{R_{S}|\operatorname{Re} \lambda|} \sup _{x \in X_{S}}\|f(x)\|,
$$

for all $\lambda \in \mathfrak{a}_{\mathrm{q}}^{*}(P, \epsilon), f \in C_{S}^{\infty}(G / H: \tau)$. To improve upon this estimate, we need the following lemma. Recall the definition of the endomorphism $\mu_{w}(D: \tau: \lambda)$ of ${ }^{\circ} \mathcal{C}_{w}(\tau)(w \in$ $\left.\mathcal{W}, D \in \mathbb{D}(G / H), \lambda \in \mathfrak{a}_{\mathrm{qc}}^{*}\right)$ from the text preceding eqn. (36).

Lemma 8.4 There exist a constant $m \in \mathbb{N}$ and for every $w \in \mathcal{W}, k \in \mathbb{N}$ and $X \in \mathfrak{a}_{\mathrm{q}}$ an $m+1$-tuple $D_{0}, \ldots, D_{m}$ in $\mathbb{D}(G / H)$ such that:

$$
\lambda(X)^{k}=\sum_{j=0}^{m} \lambda(X)^{j} \mu_{w}\left(D_{j}: \tau: \lambda\right)
$$

as an endomorphism of ${ }^{\circ} \mathcal{C}_{w}(\tau)$, for all $\lambda \in \mathfrak{a}_{\mathrm{qc}}^{*}$.

Proof. Recall the definition (14) of $\mu: \mathbb{D}(G / H) \rightarrow \mathbb{D}\left(M_{1} / H_{\mathrm{M}_{1}}\right)$, and the identity $\gamma_{\mathrm{M}_{1}} \circ \mu=$ $\gamma$ in (15). It is well known that $S(\mathfrak{b})$ is an integral ring extension of $I(\mathfrak{b})$, hence so is also $I_{\mathrm{M}_{1}}(\mathfrak{b})$, and by the identity above we conclude that $\mathbb{D}\left(M_{1} / H_{\mathrm{M}_{1}}\right)$ is an integral ring extension of $\mu(\mathbb{D}(G / H))$. Hence there exists a number $m \in \mathbb{N}$ with the property that for every $Y \in \mathfrak{a}_{\mathrm{q}} \subset \mathbb{D}\left(M_{1} / H_{\mathrm{M}_{1}}\right)$ there exist $E_{0}, \ldots, E_{m} \in \mathbb{D}(G / H)$ such that

$$
Y^{m+1}=\sum_{j=0}^{m} Y^{j} \mu\left(E_{j}\right) .
$$


Let now $w \in \mathcal{W}$, and $k \in \mathbb{N}$. Then it follows by repeated application of the above identity to $Y=\operatorname{Ad}\left(w^{-1}\right) X$ that there exist $D_{0}, \ldots, D_{m} \in \mathbb{D}(G / H)$ such that

$$
\left[\operatorname{Ad}\left(w^{-1}\right) X\right]^{k}=\sum_{j=0}^{m}\left[\operatorname{Ad}\left(w^{-1}\right) X\right]^{j} \mu\left(D_{j}\right) .
$$

Applying the isomorphism $\operatorname{Ad}(w): \mathbb{D}\left(M_{1} / H_{\mathrm{M}_{1}}\right) \rightarrow \mathbb{D}\left(M_{1} / w H_{\mathrm{M}_{1}} w^{-1}\right)$ we obtain an identity of elements in $\mathbb{D}\left(M_{1} / w H_{\mathrm{M}_{1}} w^{-1}\right)$. By (16) this may be viewed as an identity of $\mathbb{D}\left(M / w H_{\mathrm{M}} w^{-1}\right)$-valued polynomial functions on $\mathfrak{a}_{\mathrm{q} c}^{*}$. In view of (17) evaluation in $\lambda$ now results in the desired identity (69).

Completion of the proof of Proposition 8.3. For $w \in \mathcal{W}$ let $\mathcal{F}_{w}:=\operatorname{pr}_{w} \circ \mathcal{F}$. Then it suffices to prove the estimate with $\mathcal{F}$ replaced by $\mathcal{F}_{w}$.

Let $m \in \mathbb{N}$ be the constant of the above lemma. Fix $w \in \mathcal{W}, X \in \mathfrak{a}_{\mathrm{q}}^{*}, k \in \mathbb{N}$, and let $D_{0}, \ldots, D_{m} \in \mathbb{D}(G / H)$ be such that (69) holds. Then by Lemma 6.2 and (68) it follows that

$$
\begin{aligned}
\left\|\lambda(X)^{k} \pi(\lambda) \mathcal{F}_{w} f(\lambda)\right\| & \leq(1+|\lambda|)^{m} \sum_{j=0}^{m}|X|^{j}\left\|\pi(\lambda) \mathcal{F}_{w}\left(D_{j} f\right)(\lambda)\right\| \\
& \leq C^{\prime \prime}(1+|X|)^{m}(1+|\lambda|)^{m+N} e^{R_{S}|\operatorname{Re} \lambda|} \max _{j} \sup \left\|D_{j} f\right\| .
\end{aligned}
$$

Here $N$ is the constant of $(68)$, and $C^{\prime \prime}>0$ is a constant independent of $f$ and $\lambda$. It is now straightforward to complete the proof.

We shall also need an estimate for $\mathcal{F} f$ which reflects more refined aspects of the geometry of $\operatorname{supp} f$. The key to this estimate is the following estimate for the (unnormalized) Eisenstein integral.

Proposition 8.5 Let $w \in \mathcal{W}$. Then there exists a constant $C>0$ such that for all $\psi \in{ }^{\circ} \mathcal{C}_{w}(\tau), a \in A_{\mathbf{q}}$ and $\lambda \in \mathfrak{a}_{\mathrm{q}}^{*}(P, 0)-\rho_{P}$ we have

$$
\|E(P: \psi: \lambda)(a)\| \leq C \max _{v \in W_{K \cap H}} a^{v w^{-1}\left(\operatorname{Re} \lambda+\rho_{P}\right)}\|\psi\| .
$$

Proof. Since the Eisenstein integral is an Eisenstein integral for $\left(G, H_{e}\right)$ as well (see the argumentation in [6], remark after the proof of Prop. 5.6), we may assume that $H$ is connected. From [13], eqn. (69), we recall that

$$
E(P: \mathcal{L}(w) \psi: \lambda)=E\left(w^{-1} P w: \psi: w^{-1} \lambda\right),
$$

where $\mathcal{L}(w)$ is a unitary endomorphism of ${ }^{\circ} \mathcal{C}(\tau)$. From the definition of $\mathcal{L}(w)$ in loc. cit. it follows that $\mathcal{L}(w)$ maps ${ }^{\circ} \mathcal{C}_{1}(\tau)$ onto ${ }^{\circ} \mathcal{C}_{w}(\tau)$. On the other hand $\lambda \in \mathfrak{a}_{\mathrm{q}}^{*}(P, 0)-\rho_{P}$ if and only if $w^{-1} \lambda \in \mathfrak{a}_{\mathrm{q}}^{*}\left(w^{-1} P w, 0\right)-\rho_{w^{-1} P w}$; therefore it suffices to prove the result for $w=1$ and arbitrary $P$. 
Thus assume that $w=1$, and let $\psi \in{ }^{\circ} \mathcal{C}_{1}(\tau)$. Define $\tilde{\psi}(\lambda: \cdot): G / H \rightarrow V_{\tau}$ as in (28). Note that this function is continuous since $\operatorname{Re} \lambda+\rho_{P}$ is strictly $\bar{P}$-dominant, and that it vanishes outside $P H$.

Recall from (29) that the Eisenstein integral is defined by the absolutely convergent integral

$$
E(P: \psi: \lambda)(a)=\int_{K} \tau(k)^{-1} \tilde{\psi}(\lambda: k a) d k \quad\left(a \in A_{\mathrm{q}}\right) .
$$

Now fix $a \in A_{\mathrm{q}}$, and $k \in K$, and assume that $k a \in P H$. Then to complete the proof it suffices to show that

$$
\|\tilde{\psi}(\lambda: k a)\| \leq C \max _{v \in W_{K \cap H}} a^{v\left(\operatorname{Re} \lambda+\rho_{P}\right)}\|\psi\|
$$

with $C$ a constant independent of $k$ and $a$. Write $k a=n \exp X m h$, with $n \in N_{P}, X \in \mathfrak{a}_{\mathrm{q}}$, $m \in M, h \in H$. Then by the convexity theorem (Thm. 3.8) of [3] it follows that $X=Y+Z$, where $Y \in \operatorname{conv}\left(W_{K \cap H} \log a\right)$, and where $Z$ belongs to the closed convex cone spanned by the vectors $H_{\alpha}, \alpha \in \Sigma(P), m_{\alpha}^{-} \neq 0$ (here $H_{\alpha} \in \mathfrak{a}_{\mathrm{q}}$ is determined by $H_{\alpha} \perp$ ker $\alpha, \alpha\left(H_{\alpha}\right)=$ 2; cf. Section 3 for the definition of $\left.m_{\alpha}^{-}\right)$. Hence $\left(\operatorname{Re} \lambda+\rho_{P}\right)(Z) \leq 0$, and it follows that

$$
\|\tilde{\psi}(\lambda: k a)\| \leq \max _{v \in W_{K \cap H}} a^{v\left(\operatorname{Re} \lambda+\rho_{P}\right)}\|\psi(m)\| \leq \max _{v \in W_{K \cap H}} a^{v\left(\operatorname{Re} \lambda+\rho_{P}\right)}\|\psi\|_{\infty}
$$

where $\|\cdot\|_{\infty}$ denotes the sup norm on ${ }^{\circ} \mathcal{C}_{1}(\tau)=C^{\infty}\left(M / H_{\mathrm{M}}: \tau\right)$. By finite dimensionality the sup norm is equivalent to the Hilbert norm.

Corollary 8.6 Let $\delta>0, w \in \mathcal{W}$. Then there exist constants $C>0, N \in \mathbb{N}$ such that for all $\psi \in{ }^{\circ} \mathcal{C}_{w}(\tau), a \in A_{\mathbf{q}}$ and $\lambda \in \mathfrak{a}_{\mathbf{q}}^{*}(\bar{P},-\delta)-\rho_{\bar{P}}$ we have

$$
\left\|{ }^{\prime} \pi(\lambda) E^{\circ}(P: \psi: \lambda)(a)\right\| \leq C(1+|\lambda|)^{N} \max _{v \in W_{K \cap H}} a^{v w \omega^{-1}\left(\operatorname{Re} \lambda-\rho_{P}\right)}\|\psi\| .
$$

Proof. From [13], Prop. 2, we have

$$
E^{\circ}(P: \psi: \lambda)=E\left(\bar{P}: C_{P \mid \bar{P}}(1: \lambda)^{-1} \psi: \lambda\right) .
$$

In view of [7], Prop. 16.1, the $\operatorname{End}\left({ }^{\circ} \mathcal{C}(\tau)\right)$-valued function $\lambda \mapsto C_{P \mid \bar{P}}(1: \lambda)^{-1}$ is meromorphic and of $\Sigma$-polynomial growth on every set $\mathfrak{a}_{\mathrm{q}}^{*}(\bar{P}, R), R \in \mathbb{R}$. Moreover, from loc.cit.,

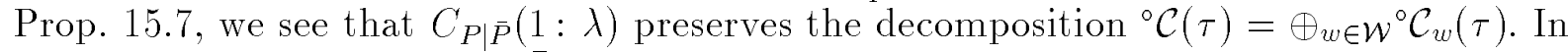
view of Proposition 8.5 with $\bar{P}$ in place of $P$ it now follows that there exist a polynomial $p \in \Pi_{\Sigma}\left(\mathfrak{a}_{\mathrm{q}}\right)$ and constants $C>0, N \in \mathbb{N}$ such that we have an estimate of the form (72), for $\lambda \in \mathfrak{a}_{\mathrm{q}}^{*}(\bar{P}, 0)-\rho_{\bar{P}}$, but with $p$ instead of ' $\pi$. The desired estimate now follows by an application of the Cauchy formula in the same way as in the proof of Lemma 6.1 in loc.cit. 
If $T$ is a closed subset of $\mathfrak{a}_{\mathrm{q}}$, we define its supporting function $h_{T}: \mathfrak{a}_{\mathrm{qc}}^{*} \rightarrow[-\infty, \infty]$ by:

$$
h_{T}(\eta)=\sup _{X \in T} \operatorname{Re} \eta(X) \quad\left(\eta \in \mathfrak{a}_{\mathrm{q}}^{*}\right) .
$$

Note that the supporting function of the empty set is given by $h_{\emptyset}=-\infty$. Note also that all values of $h_{T}$ are finite if and only if $T$ is compact and non-empty.

Corollary 8.7 Let $S$ be a $W_{K \cap H}$-invariant compact subset of $\mathfrak{a}_{\mathrm{q}}$, and let $\delta>0$. For every $n \in \mathbb{N}$ there exists a continuous seminorm $\nu$ on $C_{S}^{\infty}(G / H: \tau)$ such that for every $w \in \mathcal{W}, f \in C_{S}^{\infty}(G / H: \tau)$ and all $\lambda \in \mathfrak{a}_{\mathrm{q}}^{*}(P,-\delta)-\rho_{P}$ we have:

$$
\left\|\pi(\lambda) \mathcal{F}_{w} f(\lambda)\right\| \leq(1+|\lambda|)^{-n} e^{h_{w S}(-\lambda)} \nu(f) .
$$

Proof. Since $S$ is $W_{K \cap H}$-invariant, it follows that

$$
\sup _{\substack{a \in \exp S \\ v \in W_{K \cap H}}} a^{v w^{-1} \eta}=e^{h_{S}\left(w^{-1} \eta\right)}=e^{h_{w S}(\eta)} \quad\left(\eta \in \mathfrak{a}_{\mathrm{q}}^{*}\right) .
$$

From (42) and Corollary 8.6 it now follows in a straightforward manner that

$$
\left\|\pi(\lambda) \mathcal{F}_{w} f(\lambda)\right\| \leq C_{S}(1+|\lambda|)^{N} e^{h_{w S}(-\lambda)}\|f\|_{\infty}
$$

with $C_{S}$ a constant which only depends on $\delta$ and $S$. The proof is completed by applying Lemma 8.4 in the same fashion as in the second part of the proof of Proposition 8.3.

We now come to a lemma which is a variation on Paley-Wiener type results for Euclidean space as presented in $[32], \$ 7.3$. The lemma will enable us to collect the estimates of Proposition 8.3 and Corollary 8.7 into a simultaneous one.

Lemma 8.8 Let $S$ be a compact subset of $\mathfrak{a}_{\mathrm{q}}$, let $\epsilon, r, R>0$, and let $\phi$ be a ${ }^{\circ} \mathcal{C}(\tau)$-valued holomorphic function on $\mathfrak{a}_{\mathrm{q}}^{*}(P, \epsilon)$. Assume that for every $n \in \mathbb{N}$ we have:

(a) $\sup _{\lambda \in \mathfrak{a}_{\mathrm{q}}^{*}(P, \epsilon)}(1+|\lambda|)^{n} e^{-R|\operatorname{Re} \lambda|}\|\phi(\lambda)\|<\infty$;

(b) $\sup _{\lambda \in \mathfrak{a}_{\mathrm{q}}^{*}(P,-r)}(1+|\lambda|)^{n} e^{-h_{S}(-\lambda)}\|\phi(\lambda)\|<\infty$.

Then for each $N \in \mathbb{N}$ we have:

$$
\sup _{\lambda \in \overline{\mathfrak{a}}_{\mathrm{q}}^{*}(P, 0)}(1+|\lambda|)^{N} e^{-h_{S}(-\lambda)}\|\phi(\lambda)\|<\infty .
$$

Moreover, the seminorm in the latter expression can be estimated from above by a constant times the seminorm in (a) with a suitable $n$ (depending on $N$ ).

Proof. Let $\nu_{n}(\phi)$ denote the seminorm in (a). From the estimates in (a) it follows that the restriction to $i \mathfrak{a}_{\mathrm{q}}^{*}$ of $\phi$ is a Schwartz function; in fact by a straightforward application 
of Cauchy's integral formula we see that every Schwartz-type seminorm can be estimated from above by (a constant times) one of the seminorms $\nu_{n}(\phi)$.

Let $f: \mathfrak{a}_{\mathrm{q}} \rightarrow{ }^{\circ} \mathcal{C}(\tau)$ be the inverse Fourier transform of $\phi$, defined by

$$
f(x)=\int_{i \mathfrak{a}_{\mathrm{q}}^{*}} e^{\lambda(x)} \phi(\lambda) d \lambda
$$

Then $f$ is a Schwartz function on the Euclidean space $\mathfrak{a}_{\mathrm{q}}$, and by continuity of the inverse Fourier tranfsorm for the Schwartz topologies every Schwartz-seminorm of $f$ can be estimated by one of the $\nu_{n}(\varphi)$. Moreover, it follows from the Euclidean Fourier inversion formula that with a suitably normalized Lebesgue measure $d x$ on $\mathfrak{a}_{\mathrm{q}}$ we have

$$
\phi(\lambda)=\int_{\mathfrak{a}_{\mathrm{q}}} e^{-\lambda(x)} f(x) d x
$$

for $\lambda \in i \mathfrak{a}_{\mathrm{q}}^{*}$.

Let $\lambda_{0} \in \mathfrak{a}_{\mathbf{q}}^{*}(P, 0)$, then it follows from (75) and an application of Cauchy's theorem, justified by (a), that

$$
f(x)=\int_{i \mathfrak{a}_{\mathrm{q}}^{*}} e^{\lambda_{0}(x)+\lambda(x)} \phi\left(\lambda_{0}+\lambda\right) d \lambda .
$$

In particular, this shows that $x \mapsto e^{-\lambda_{0}(x)} f(x)$ is a Schwartz function, and hence also that we have $(76)$ for $\lambda \in \mathfrak{a}_{\mathrm{q}}^{*}(P, 0)$.

Let $t>0$ be any number such that $t \lambda_{0} \in \mathfrak{a}_{\mathrm{q}}^{*}(P,-r)$. Replacing $\lambda_{0}$ by $t \lambda_{0}$ in (77) and applying (b) with a suitably large $n$ we obtain the estimate

$$
\|f(x)\| \leq C e^{t \operatorname{Re} \lambda_{0}(x)} e^{h_{S}\left(-t \lambda_{0}\right)} \int_{i_{\mathfrak{a}_{\mathrm{q}}^{*}}^{*}}\left(1+\left|t \lambda_{0}+\lambda\right|\right)^{-n} d \lambda
$$

with $C$ a positive constant. By taking the limit as $t \rightarrow \infty$ we infer that if $\operatorname{Re} \lambda_{0}(x)+$ $h_{S}\left(-\lambda_{0}\right)<0$ then $f(x)=0$.

In (76) we now need only integrate over the set where $-\operatorname{Re} \lambda_{0}(x) \leq h_{S}\left(-\lambda_{0}\right)$ for all $\lambda_{0} \in \mathfrak{a}_{\mathrm{q}}^{*}(P, 0)$, and hence the integrand $f(x) e^{-\lambda(x)}$ is dominated by $e^{h_{S}(-\lambda)}\|f(x)\|$, if $\lambda \in \mathfrak{a}_{\mathrm{q}}^{*}(P, 0)$; by continuity this domination still holds if $\lambda$ belongs to the closure of $\mathfrak{a}_{\mathrm{q}}^{*}(P, 0)$. Thus we obtain

$$
\|\phi(\lambda)\| \leq e^{h_{S}(-\lambda)} \int_{\mathfrak{a}_{\mathrm{q}}}\|f(x)\| d x
$$

valid for all $\lambda \in \overline{\mathfrak{a}}_{\mathrm{q}}^{*}(P, 0)$. A similar estimate is obtained for $\lambda\left(x_{0}\right)^{k} \phi(\lambda)$ for any $x_{0} \in \mathfrak{a}_{\mathrm{q}}$, $k \in \mathbb{N}$; on the right-hand side $f$ is then replaced by its $k$-th derivative in the direction $x_{0}$. This shows that the seminorm in (74) can be estimated in terms of a Schwartz-seminorm of $f$, which in turn may be estimated by $\nu_{n}(\phi)$, for a suitable $n$.

In view of Lemma 8.8 , Proposition 8.3 and Corollary 8.7 provide motivation for the following definition of pre Paley-Wiener spaces. If $\varphi$ is a ${ }^{\circ} \mathcal{C}(\tau)$-valued function, we write $\varphi_{w}=\operatorname{pr}_{w} \circ \varphi$ for its $w$-component, $w \in \mathcal{W}$. 
Definition 8.9 Let $S$ be a non-empty $W_{K \cap H}$-invariant compact subset of $\mathfrak{a}_{\mathrm{q}}$. If $p \in \Pi\left(\mathfrak{a}_{\mathrm{q}}\right)$, we define the pre Paley-Wiener space $\mathcal{M}(S, p)$ to be the space of meromorphic functions $\varphi: \mathfrak{a}_{\mathrm{q} c}^{*} \rightarrow{ }^{\circ} \mathcal{C}(\tau)$ satisfying the following three conditions $(\mathrm{a})-(\mathrm{c})$ :

(a) For each $s \in W$ we have $\varphi(s \lambda)=C_{P \mid P}^{\circ}(s: \lambda) \varphi(\lambda)$ as a meromorphic identity in $\lambda$;

(b) The function $p \varphi$ is holomorphic on an open neighborhood of $\overline{\mathfrak{a}}_{\mathbf{q}}^{*}(P, 0)$;

(c) For every $n \in \mathbb{N}$ and each $w \in \mathcal{W}$ we have:

$$
\sup _{\lambda \in \overline{\mathfrak{a}}_{\mathrm{q}}^{*}(P, 0)}(1+|\lambda|)^{n} e^{-h_{w S}(-\lambda)}\left\|p(\lambda) \varphi_{w}(\lambda)\right\|<\infty
$$

The pre Paley-Wiener space $\mathcal{M}(S, p)$ is equipped with the structure of a locally convex space by means of the seminorms in condition (c).

Remark 8.10 In addition to the above we adopt the convention that $\mathcal{M}(\emptyset, p)$ is the trivial space, for every $p \in \Pi\left(\mathfrak{a}_{\mathrm{q}}\right)$.

Note that, if $S \subset S^{\prime}$ and if $p$ divides $p^{\prime}$, we have $\mathcal{M}(S, p) \subset \mathcal{M}\left(S^{\prime}, p^{\prime}\right)$, the inclusion map being continuous.

One can actually prove that $\mathcal{M}(S, p)$ is a Fréchet space. We will do this at a later stage, under the assumption that $p \in \Pi_{\Sigma}\left(\mathfrak{a}_{\mathrm{q}}\right)$, see Corollary 20.2.

The results obtained in this section can now be summarized in the following theorem.

Theorem 8.11 Let $\pi \in \Pi_{\Sigma}\left(\mathfrak{a}_{\mathrm{q}}\right)$ be as in (64). Let $S$ be a $W_{K \cap H}$-invariant compact subset of $\mathfrak{a}_{\mathrm{q}}$. Then $\mathcal{F}$ maps $C_{S}^{\infty}(G / H: \tau)$ continuously into $\mathcal{M}(S, \pi)$.

Proof. Let $f \in C_{S}^{\infty}(G / H: \tau)$. The meromorphic function $\varphi=\mathcal{F} f$ satisfies (a) in view of Lemma 6.3 with $P_{1}=P_{2}=P$, and (b) in view of (64) and Lemma 8.1 (b). Moreover, from Proposition 8.3 and Corollary 8.7 it follows by application of Lemma 8.8 that $\varphi$ satisfies (c).

\section{Wave packets}

In this section $P$ is a fixed element of $\mathcal{P}_{\sigma}^{\min }$. For $n \in \mathbb{N}$ let $C_{n}\left(i \mathfrak{a}_{\mathrm{q}}^{*}\right)$ denote the space of continuous functions $\varphi: i \mathfrak{a}_{\mathrm{q}}^{*} \rightarrow \mathbb{C}$ such that

$$
\sup _{\lambda \in i \mathfrak{a}_{\mathrm{q}}^{*}}(1+|\lambda|)^{n}|\varphi(\lambda)|<\infty
$$

equipped with the obvious structure of a Banach space. Then

$$
C_{\mathbb{N}}\left(i \mathfrak{a}_{\mathrm{q}}^{*}\right):=\bigcap_{n \in \mathbb{N}} C_{n}\left(i \mathfrak{a}_{\mathrm{q}}^{*}\right)
$$


is a Fréchet space in a natural way. For $\varphi \in C_{\mathbb{N}}\left(i \mathfrak{a}_{\mathrm{q}}^{*}\right) \otimes{ }^{\circ} \mathcal{C}(\tau)$, we define the wave packet $\mathcal{J} \varphi=\mathcal{J}_{P} \varphi$ by

$$
\mathcal{J} \varphi(x)=\int_{i \mathfrak{a}_{\mathrm{q}}^{*}} E^{\circ}(P: \varphi(\lambda): \lambda)(x) d \lambda \quad(x \in G / H) .
$$

The integral converges absolutely by the estimates of [7], Thm. 19.2, which hold with $\pi=1$, in view of the regularity theorem (Proposition 5.4). Obviously $\mathcal{J} \varphi$ is a $\tau$-spherical function on $G / H$.

If $\varphi \in C_{\mathbb{N}}\left(i \mathfrak{a}_{\mathrm{q}}^{*}\right) \otimes{ }^{\circ} \mathcal{C}(\tau)$ and $D \in \mathbb{D}(G / H)$, then we write $\mu(D: \tau) \varphi$ for the function $\lambda \mapsto$ $\mu(D: \tau: \lambda) \varphi(\lambda)$. Notice that $\varphi \mapsto p \varphi$ is a continuous linear endomorphism of $C_{\mathbb{N}}\left(i \mathfrak{a}_{\mathrm{q}}^{*}\right) \otimes$ ${ }^{\circ} \mathcal{C}(\tau)$ for any $\operatorname{End}\left({ }^{\circ} \mathcal{C}(\tau)\right)$-valued polynomial $p$ on $i \mathfrak{a}_{\mathrm{q}}^{*}$, hence in particular for $p=\mu(D: \tau)$.

Lemma 9.1 The wave packet map $\mathcal{J}$ is a continuous linear map from $C_{\mathbb{N}}\left(i \mathfrak{a}_{\mathrm{q}}^{*}\right) \otimes{ }^{\circ} \mathcal{C}(\tau)$ to $C^{\infty}(G / H: \tau)$. Moreover, for all $\varphi \in C_{\mathbb{N}}\left(i \mathfrak{a}_{\mathrm{q}}^{*}\right) \otimes{ }^{\circ} \mathcal{C}(\tau)$ and $D \in \mathbb{D}(G / H)$ we have:

$$
D \mathcal{J} \varphi=\mathcal{J}(\mu(D: \tau) \varphi)
$$

Proof. The first assertion is a straightforward consequence of the above mentioned estimates of [7], Thm. 19.2. In fact, it follows from these estimates that differentiation under the integral sign is allowed in $(78)$ : if $u \in U(\mathfrak{g})$, then we have

$$
L_{u} \mathcal{J} \varphi(x)=\int_{i \mathfrak{a}_{\mathrm{q}}^{*}} L_{u}\left[E^{\circ}(\varphi(\lambda): \lambda)\right](x) d \lambda .
$$

Let now $v$ be a representative of $D$ in $U(\mathfrak{g})^{H}$, and let $x \in G$ be fixed. Then $D \mathcal{J} \varphi(x)=$ $R_{v}(\mathcal{J} \varphi)(x)=L_{u}(\mathcal{J} \varphi)(x)$, with $u=[\operatorname{Ad}(x) v]^{\vee}$, where $y \mapsto y^{\vee}$ denotes the principal antiautomorphism of $U(\mathfrak{g})$. Inserting this in $(80)$, and observing that $L_{u}\left[E^{\circ}(\varphi(\lambda): \lambda)\right](x)=$ $D\left[E^{\circ}(\varphi(\lambda): \lambda)\right](x)=E^{\circ}(\mu(D: \tau: \lambda) \varphi(\lambda): \lambda)(x)$ for all $\lambda$, we obtain $(79)$.

Remark 9.2 Notice that if $p$ has no zeros on $i \mathfrak{a}_{\mathrm{q}}^{*}$ then $\varphi \in \mathcal{M}(S, p)$ implies that $\left.\varphi\right|_{i \mathfrak{a}_{\mathrm{q}}^{*}} \in$ $C_{\mathbb{N}}\left(i \mathfrak{a}_{\mathrm{q}}^{*}\right)$, for all $S$ and $p$ as in Definition 8.9 , and the map $\left.\varphi \mapsto \varphi\right|_{i \mathfrak{a}_{\mathrm{q}}^{*}}$ is continuous. Hence in this case $\mathcal{J}$ gives rise to a continuous transformation, also denoted $\mathcal{J}$, from $\mathcal{M}(S, p)$ to $C^{\infty}(G / H: \tau)$. In particular this applies to the polynomial $\pi$ defined in $(64)$.

We will now discuss the relation of the wave packet transform to the spherical Fourier transform.

Recall the definition of the functions $\Theta, \tau_{\mathrm{G} / \mathrm{H}}: G / H \rightarrow[0, \infty[$ from Section 6 . For $p, n \in \mathbb{N}$, let $T_{n}^{p}(G / H: \tau)$ be the Banach space of functions $f \in C^{p}(G / H: \tau)$, such that

$$
\sup _{x \in G / H}\left(1+\tau_{\mathrm{G} / \mathrm{H}}(x)\right)^{-n} \Theta(x)^{-1}\left\|L_{u} f(x)\right\|<\infty,
$$

for all $u \in U(\mathfrak{g})$ with order $u \leq p$. Then by [7], Cor. 17.6 we have a $G$-equivariant, continuous sesquilinear pairing $\langle\cdot \mid \cdot\rangle: T_{n}^{p}(G / H: \tau) \times \mathcal{C}(G / H: \tau) \rightarrow \mathbb{C}$, defined by

$$
\langle f \mid g\rangle:=\int_{G / H}\langle f(x) \mid g(x)\rangle_{V_{\tau}} d x .
$$


Since the inclusion map $\mathcal{S}\left(i \mathfrak{a}_{\mathrm{q}}^{*}\right) \rightarrow C_{\mathbb{N}}\left(i \mathfrak{a}_{\mathrm{q}}^{*}\right)$ is obviously continuous, Lemma 6.1 shows that the spherical Fourier transform $\mathcal{F}$ maps $\mathcal{C}(G / H: \tau)$ continuously to $C_{\mathbb{N}}\left(i \mathfrak{a}_{\mathrm{q}}^{*}\right) \otimes{ }^{\circ} \mathcal{C}(\tau)$. We equip the latter space with the continuous sesquilinear pairing

$$
\langle\varphi \mid \psi\rangle=\int_{i \mathfrak{a}_{\mathrm{q}}^{*}}\langle\varphi(\lambda) \mid \psi(\lambda)\rangle_{\circ} \mathcal{C}(\tau) d \lambda \quad\left(\varphi, \psi \in C_{\mathbb{N}}\left(i \mathfrak{a}_{\mathrm{q}}^{*}\right) \otimes{ }^{\circ} \mathcal{C}(\tau)\right) .
$$

Lemma 9.3 For every $p \in \mathbb{N}$ there exists a number $n \in \mathbb{N}$ such that $\mathcal{J}$ is a continuous linear map from $C_{\mathbb{N}}\left(i \mathfrak{a}_{\mathrm{q}}^{*}\right) \otimes{ }^{\circ} \mathcal{C}(\tau)$ to $T_{n}^{p}(G / H: \tau)$. Moreover, $\mathcal{J}$ is the transposed of $\mathcal{F}$ in the sense that

$$
\langle\mathcal{J} \varphi \mid f\rangle=\langle\varphi \mid \mathcal{F} f\rangle \quad\left(\varphi \in C_{\mathbb{N}}\left(i \mathfrak{a}_{\mathrm{q}}^{*}\right) \otimes{ }^{\circ} \mathcal{C}(\tau), f \in \mathcal{C}(G / H: \tau)\right)
$$

Proof. The first part of the lemma is a straightforward consequence of (80) and the estimates in [7], Thm. 19.2. Moreover, by the same estimates it follows that given $\varphi$ and $f$ as in (82) the function $\Phi: i \mathfrak{a}_{\mathrm{q}}^{*} \times G / H \rightarrow \mathbb{C}$, defined by

$$
\Phi(\lambda, x)=\left\langle E^{\circ}(\varphi(\lambda): \lambda)(x) \mid f(x)\right\rangle_{V_{\tau}}
$$

is an absolutely integrable function with respect to the product measure $d \lambda d x$ on $i \mathfrak{a}_{\mathrm{q}}^{*} \times$ $G / H$. Integration of $\Phi(\lambda, x)$ first with respect to $\lambda$ and then with respect to $x$ yields the left-hand side of (82); integration in the reversed order yields the right-hand side. In view of Fubini's theorem this implies (82).

\section{Corollary 9.4}

(a) The composition $\mathcal{J} \mathcal{F}$ is a continuous linear map from $\mathcal{C}(G / H: \tau)$ to $C^{\infty}(G / H: \tau)$, which commutes with the action of $\mathbb{D}(G / H)$.

(b) There exists a number $n \in \mathbb{N}$ such that $\mathcal{J} \mathcal{F}$ is a continuous linear map from $\mathcal{C}(G / H: \tau)$ to $T_{n}^{0}(G / H: \tau)$. The map $\mathcal{J} \mathcal{F}$ is formally symmetric in the sense that for all $f, g \in \mathcal{C}(G / H: \tau)$ we have:

$$
\langle\mathcal{J} \mathcal{F} f \mid g\rangle=\langle f \mid \mathcal{J} \mathcal{F} g\rangle
$$

Proof. This is an immediate consequence of Lemmas 6.1, 6.2, 9.1 and 9.3. 


\section{Support properties of wave packets}

For the Euclidean and the non-compact Riemannian symmetric spaces, the transformation $\mathcal{J}$ is also the inverse of the Fourier transformation $\mathcal{F}$, and the Paley-Wiener theorem is derived from the fact that if $\varphi$ extends to a holomorphic function on $\mathfrak{a}_{\mathrm{qc}}^{*}$ of exponential growth, then $\mathcal{J} \varphi$ has bounded support. In the more general case of a semisimple symmetric space, the situation is more complicated, primarily because $E^{\circ}(\psi: \lambda)$ is only meromorphic in $\lambda$. This is one of the reasons we can only derive conclusions about the support of $D \mathcal{J} \varphi=\mathcal{J} \mu(D: \tau) \varphi$, with $D$ a suitable operator from $\mathbb{D}(G / H)$ (depending on $\tau)$.

For $Q \in \mathcal{P}_{\sigma}^{\min }$, we denote by $c_{\mathbf{q}}^{+}(Q)$ the closed dual cone of $\mathfrak{a}_{\mathbf{q}}^{+}(Q)$ in $\mathfrak{a}_{\mathrm{q}}$ :

$$
c_{\mathrm{q}}^{+}(Q):=\left\{X \in \mathfrak{a}_{\mathrm{q}} \mid \forall Y \in \mathfrak{a}_{\mathrm{q}}^{+}(Q):\langle X, Y\rangle \geq 0\right\} .
$$

Notice that we also have:

$$
\mathfrak{a}_{\mathrm{q}}^{+}(Q)=\left\{Y \in \mathfrak{a}_{\mathrm{q}} \mid \forall X \in c_{\mathrm{q}}^{+}(Q):\langle X, Y\rangle>0\right\} .
$$

Let $T$ be a closed subset of $\mathfrak{a}_{\mathrm{q}}$. Then its supporting function $h_{T}: \mathfrak{a}_{\mathrm{qc}}^{*} \rightarrow[-\infty, \infty]$, defined by (73), is lower semi-continuous. If $T$ is non-empty and compact, then $h_{T}$ is finite and continuous.

The supporting function will be useful to us because of the well known fact that the closure of the convex hull of $T$ is given by

$$
\operatorname{cl}(\operatorname{conv} T)=\left\{Y \in \mathfrak{a}_{\mathrm{q}} \mid \eta(Y) \leq h_{T}(\eta) \quad\left(\forall \eta \in \mathfrak{a}_{\mathrm{q}}^{*}\right)\right\}
$$

Indeed this follows immediately from the fact that every point in the complement of cl $(\operatorname{conv} T)$ may be separated from $T$ by a hyperplane.

The following lemma will be needed in the rest of this section. If $S, S^{\prime}$ are subsets of $\mathfrak{a}_{\mathrm{q}}$, we write $S-S^{\prime}=\left\{X-X^{\prime} \mid X \in S, X^{\prime} \in S^{\prime}\right\}$.

Lemma 10.1 Let $S \subset \mathfrak{a}_{\mathrm{q}}$ be compact and convex, and let $Q \in \mathcal{P}_{\sigma}^{\min }$. Then

(a) $S-c_{\mathrm{q}}^{+}(Q)=\left\{Y \in \mathfrak{a}_{\mathrm{q}} \mid \forall \eta \in \mathfrak{a}_{\mathrm{q}}^{*} \cap \mathfrak{a}_{\mathrm{q}}^{*}(\bar{Q}, 0): \eta(Y) \leq h_{S}(\eta)\right\}$;

(b) $\left[S-c_{\mathrm{q}}^{+}(Q)\right] \cap \mathfrak{a}_{\mathrm{q}}^{+}(Q) \subset \operatorname{conv} W S$.

Proof. If $S=\emptyset$, then the assertions are obvious. Therefore we assume that $S$ is non-empty. Notice that

$$
c_{\mathrm{q}}^{+}(Q)=\left\{Y \in \mathfrak{a}_{\mathrm{q}} \mid \forall \eta \in \mathfrak{a}_{\mathrm{q}}^{*} \cap \mathfrak{a}_{\mathrm{q}}^{*}(\bar{Q}, 0): \eta(Y) \geq 0\right\} .
$$

It is now straightforward to check that $T:=S-c_{\mathrm{q}}^{+}(Q)$ is contained in the right-hand side of (a). Conversely, let $Y \in \mathfrak{a}_{\mathrm{q}}$ belong to the right-hand side of (a). To prove that $Y \in T$, it suffices to show that

$$
\nu(Y) \leq h_{T}(\nu) \quad\left(\forall \nu \in \mathfrak{a}_{\mathrm{q}}^{*}\right)
$$


since $T$ is closed and convex (see (85)). Now $h_{S} \leq h_{T}$ since $S \subset T$. Hence if $\nu \in \mathfrak{a}_{\mathrm{q}}^{*}$ is strictly $Q$-dominant then (86) follows from the assumption on $Y$. If $\nu$ is $Q$-dominant, this is still the case, since $h_{S}$ is continuous. On the other hand, if $\nu$ is not $Q$-dominant, then $\nu\left(c_{\mathrm{q}}^{+}(Q)\right)$ is not bounded from below, hence $h_{T}(\nu)=\infty$, and (86) follows.

It remains to prove (b). Let $Y \in T \cap \mathfrak{a}_{\mathfrak{q}}^{+}(Q)$. Then by (85) it suffices to prove that for every $\nu \in \mathfrak{a}_{\mathrm{q}}^{*}$ one has

$$
\nu(Y) \leq h_{W S}(\nu) .
$$

Fix $\nu \in \mathfrak{a}_{\mathrm{q}}^{*}$. Then there exists a $w \in W$ such that $w^{-1} \nu$ is $Q$-dominant, and by (a) there exists an element $X \in S$ such that $w^{-1} \nu(X) \geq w^{-1} \nu(Y)$. But then

$$
\nu(w X)=w^{-1} \nu(X) \geq w^{-1} \nu(Y) \geq \nu(Y) ;
$$

indeed the last inequality holds because $Y \in \mathfrak{a}_{\mathrm{q}}^{+}(Q)$, whereas $w^{-1} \nu$ is $Q$-dominant, so that $Y-w^{-1} Y \in c_{\mathbf{q}}^{+}(Q)$ and $w^{-1} \nu\left(Y-w^{-1} Y\right) \geq 0$. This proves $(87)$.

The main result of this section is the following theorem. See Definition 8.9 for the definition of the pre Paley-Wiener space $\mathcal{M}(S, p)$. Let $P \in \mathcal{P}_{\sigma}^{\min }$ and $\mathcal{J}=\mathcal{J}_{P}$.

Theorem 10.2 Let $p \in \Pi\left(\mathfrak{a}_{\mathrm{q}}\right)$ and $w \in \mathcal{W}$. Then there exists a differential operator $D \in \mathbb{D}(G / H)$, such that

(a) $p$ divides $\mu(D: \tau)$;

(b) $\operatorname{det} \mu(D: \tau) \in \Pi\left(\mathfrak{a}_{\mathrm{q}}\right)$;

(c) for every $W_{K \cap H}$-invariant convex compact subset $S$ of $\mathfrak{a}_{q}$, and for all $\varphi \in \mathcal{M}(S, p)$ we have

$$
\operatorname{supp} \mathcal{J}[\mu(D: \tau) \varphi] \cap A_{\mathrm{q}}^{+}\left(w^{-1} P w\right) \subset \exp \left[S-c_{\mathrm{q}}^{+}\left(w^{-1} P w\right)\right]
$$

Notice that by (a) we have $\mu(D: \tau) \varphi \in C_{\mathbb{N}}\left(i \mathfrak{a}_{\mathrm{q}}^{*}\right)$ for all $\varphi \in \mathcal{M}(S, p)$, so that the application of $\mathcal{J}$ in (88) makes sense. Furthermore, here and below we regard the chambers $A_{\mathrm{q}}^{+}(Q), Q \in \mathcal{P}_{\sigma}^{\mathrm{min}}$, as subsets of $G / H$ by means of the projection $G \rightarrow G / H$.

Before starting with the proof of this theorem we first deduce a corollary from it.

Definition 10.3 Let $p \in \Pi\left(\mathfrak{a}_{q}\right)$. Then we define $\mathbb{D}_{p}=\mathbb{D}_{\tau, p}$ to be the set of $D \in \mathbb{D}(G / H)$ such that $D^{*}=D$ and

(a) $p$ divides $\mu(D: \tau)$;

(b) $\operatorname{det} \mu(D: \tau) \in \Pi\left(\mathfrak{a}_{\mathrm{q}}\right)$;

(c) for every $W_{K \cap H}$-invariant convex compact subset $S \subset \mathfrak{a}_{\mathrm{q}}$ and for all $\varphi \in \mathcal{M}(S, p)$ we have

$$
\operatorname{supp} \mathcal{J}[\mu(D: \tau) \varphi] \cap A_{\mathrm{q}}^{+}(Q) \subset \exp \left[S-c_{\mathrm{q}}^{+}(Q)\right] \quad\left(Q \in \mathcal{P}_{\sigma}^{\min }\right)
$$


Corollary 10.4 Let $p \in \Pi\left(\mathfrak{a}_{q}\right)$. Then $\mathbb{D}_{p} \neq \emptyset$. Moreover, if $D \in \mathbb{D}_{p}$ then for every $W$ invariant convex compact subset $S \subset \mathfrak{a}_{\mathrm{q}}$ we have $\mathcal{J}[\mu(D: \tau) \varphi] \in C_{S}^{\infty}(G / H: \tau)$ for all $\varphi \in$ $\mathcal{M}(S, p)$, and the map $\varphi \mapsto \mathcal{J}[\mu(D: \tau) \varphi]$ is continuous from $\mathcal{M}(S, p)$ to $C_{S}^{\infty}(G / H: \tau)$.

Remark 10.5 If $p$ has no zeros on $i \mathfrak{a}_{\mathrm{q}}^{*}$, then from Remark 9.2 and Lemma 9.1 it follows that $\mathcal{J} \mu(D: \tau) \varphi=D \mathcal{J} \varphi$, for all $\varphi \in \mathcal{M}(S, p)$. In particular this applies to the polynomial $\pi$ defined in (64).

Proof of Corollary 10.4. For $w \in \mathcal{W}$, let $D_{w} \in \mathbb{D}(G / H)$ fulfill the requirements of Theorem 10.2, and put

$$
D_{0}=\prod_{w \in \mathcal{W}} D_{w}, \quad D=D_{0}^{*} D_{0} .
$$

We will first show that $D \in \mathbb{D}_{p}$. It is readily verified that $D^{*}=D$ and that condition (a) of Definition 10.3 holds. Condition (b) follows because by (40) we have

$$
\operatorname{det} \mu\left(D_{0}^{*}: \tau: \lambda\right)=\operatorname{det} \mu\left(D_{0}: \tau:-\bar{\lambda}\right)^{*}=\overline{\operatorname{det} \mu\left(D_{0}: \tau:-\bar{\lambda}\right)} .
$$

To see that (c) holds, let $S \subset \mathfrak{a}_{\mathrm{q}}$ be a $W_{K \cap H}$-invariant convex compact subset, and let $\varphi \in$ $\mathcal{M}(S, p)$. Then for every $w \in \mathcal{W}$ we have $\mathcal{J}[\mu(D: \tau) \varphi]=D_{0}^{*} \prod_{v \in \mathcal{W}, v \neq w} D_{v} \mathcal{J}\left[\mu\left(D_{w}: \tau\right) \varphi\right]$ by (79), and hence supp $\mathcal{J}[\mu(D: \tau) \varphi] \subset \operatorname{supp} \mathcal{J}\left[\mu\left(D_{w}: \tau\right) \varphi\right]$. Thus we see that $(88)$ holds for the present $D$ and each $w \in \mathcal{W}$. If $u \in N_{K \cap H}\left(\mathfrak{a}_{q}\right)$, then $\mathcal{J}[\mu(D: \tau) \varphi]\left(u a u^{-1}\right)=$ $\tau(u) \mathcal{J}[\mu(D: \tau) \varphi](a)$, and $\exp \left[S-c_{\mathrm{q}}^{+}\left(u^{-1} w^{-1} P w u\right)\right]=u^{-1} \exp \left[S-c_{\mathrm{q}}^{+}\left(w^{-1} P w\right)\right] u$, and we see that $(88)$ holds for all $w \in \mathcal{W} N_{K \cap H}\left(\mathfrak{a}_{\mathrm{q}}\right)$. The latter set is naturally mapped onto $W$, and since $W P=\mathcal{P}_{\sigma}^{\min },(89)$ follows. Hence $\mathbb{D}_{p} \neq \emptyset$.

To prove the remaining assertions, let $D \in \mathbb{D}_{p}$, and let $S \subset \mathfrak{a}_{\mathrm{q}}$ satisfy the hypothesis. Then from (89) and Lemma 10.1 (b) we obtain that

$$
\operatorname{supp} \mathcal{J}[\mu(D: \tau) \varphi] \cap A_{\mathrm{q}}^{+}(Q) \subset \exp S,
$$

for all $Q \in \mathcal{P}_{\sigma}^{\min }$, and since $G / H=\mathrm{cl} \cup_{Q \in \mathcal{P}_{\sigma}^{\min }} K A_{\mathrm{q}}^{+}(Q) H$, we infer that $\mathcal{J}[\mu(D: \tau) \varphi] \in$ $C_{S}^{\infty}(G / H: \tau)$. Finally the map $\varphi \mapsto \mu(D: \tau) \varphi$ is continuous from $\mathcal{M}(S, p)$ to $C_{\mathbb{N}}\left(i \mathfrak{a}_{\mathrm{q}}^{*}\right)$ since $p$ divides $\mu(D: \tau)$, and hence the asserted continuity follows from Lemma 9.3.

For the proof of Theorem 10.2 we need some preparation. We recall from (19) that $\mathfrak{j}=\mathfrak{j}_{0} \oplus \mathfrak{a}_{\mathbf{q}}$ is a Cartan subalgebra of $\mathfrak{g}$. Let $W_{\mathbf{n}}(\mathfrak{j})$ denote the normalizer of $\mathfrak{a}_{\mathbf{q}}$ in $W(\mathfrak{j})$.

Lemma 10.6 Let $X \in \mathfrak{a}_{\mathrm{q}} \backslash\{0\}$. Then there exists an element $Y \in \mathfrak{j}_{0}$ such that $X+Y \notin$ $w\left(\mathbf{j}_{0 \mathbf{c}}\right)$ for each $w \in W(\mathfrak{j})$.

Proof. If $w \in W_{\mathbf{n}}(\mathfrak{j})$, then $w$ also normalizes $\mathfrak{j}_{0}$. Hence in this case $X+Y \notin w\left(\mathfrak{j}_{0 \mathbf{c}}\right)$ for any $Y \in \mathfrak{j}_{0}$.

On the other hand, if $w \in W(\mathfrak{j}) \backslash W_{\mathbf{n}}(\mathfrak{j})$, then we may select $\nu_{w} \in \mathfrak{a}_{\mathbf{q}}^{*}$ such that $w \nu_{w} \notin \mathfrak{a}_{\mathbf{q}}^{*}$. Thus $p_{w}: Y \mapsto w \nu_{w}(X+Y)$ is a non-trivial polynomial function on $\mathbf{j}_{0}$.

Let now $Y \in \mathfrak{j}_{0}$ be such that $p_{w}(Y) \neq 0$ for every $w \in W(\mathfrak{j}) \backslash W_{\mathbf{n}}(\mathfrak{j})$. Then for each $w \in W(\mathfrak{j}) \backslash W_{\mathbf{n}}(\mathfrak{j})$ we have: $w \nu_{w}(X+Y) \neq 0$. Since $\nu_{w} \in \mathfrak{a}_{\mathrm{q}}^{*}$, this implies that $X+Y \notin w\left(\mathbf{j}_{0 \mathbf{c}}\right)$. 
Lemma 10.7 Let $p \in \Pi\left(\mathfrak{a}_{\mathrm{q}}\right)$. Then there exists a $D \in \mathbb{D}(G / H)$ such that $p \otimes I^{\circ} \mathcal{C}(\tau)$ divides $\mu(D: \tau)$ in $S\left(\mathfrak{a}_{\mathrm{q}}\right) \otimes \operatorname{End}\left({ }^{\circ} \mathcal{C}(\tau)\right)$, and such that det $\mu(D: \tau)$ belongs to $\Pi\left(\mathfrak{a}_{\mathrm{q}}\right)$.

Remark 10.8 In the proof below we will see that in fact $D$ can be chosen from $\mathbb{Z}(G / H)=$ $r(\mathfrak{Z})$.

Proof of Lemma 10.7. Since $D \mapsto \mu(D: \tau)$ is an algebra homomorphism from $\mathbb{D}(G / H)$ into $S\left(\mathfrak{a}_{\mathrm{q}}\right) \otimes \operatorname{End}\left({ }^{\circ} \mathcal{C}(\tau)\right)$, it suffices to prove the lemma for $p=X+c$, with $X \in \mathfrak{a}_{\mathrm{qc}} \backslash\{0\}$ and $c \in \mathbb{C}$. Let $Y \in \mathfrak{j}_{0}$ be associated with $X$ as in the previous lemma. For each $[\xi] \in \widehat{M}_{H}$ let $\Lambda_{\xi} \in \mathfrak{j}_{0 \mathbf{c}}^{*}$ denote the infinitesimal character of $\xi$. Recall the finite decomposition (27) of ${ }^{\circ} \mathcal{C}(\tau)$ and define

$$
q(\Lambda)=\prod_{\substack{w \in W(\mathrm{j}) \\\left[\xi^{\vee}\right] 1 \tau}}\left[\left(w \Lambda+\Lambda_{\xi}\right)(X+Y)+c\right] \quad\left(\Lambda \in \mathfrak{j}_{\mathbf{c}}^{*}\right)
$$

Then $q \in S(\mathfrak{j})^{W(\mathfrak{j})}$, hence $q$ equals the image $\gamma_{\mathfrak{j}}^{\mathfrak{g}}(Z)$ of a unique element $Z \in \mathfrak{Z}$ under the Harish-Chandra isomorphism $\gamma_{j}^{\mathfrak{g}}$. We claim that $D=r(Z)$ satisfies the requirements. For this let $[\xi] \in \widehat{M}_{H}$ and notice that by Lemma 5.7 the endomorphism $\mu(r(Z): \tau: \lambda)(\lambda \in$ $\left.\mathfrak{a}_{\mathrm{qc}}^{*}\right)$ acts on ${ }^{\circ} \mathcal{C}_{\xi}(\tau)$ by multiplication with $\gamma_{j}^{\mathfrak{g}}\left(Z: \lambda-\Lambda_{\xi}\right)$, that is by

$$
q\left(\lambda-\Lambda_{\xi}\right)=\prod_{\substack{w \in W(j) \\\left[\xi^{\prime}\right] \uparrow \tau}}\left[\left(w \lambda-w \Lambda_{\xi}+\Lambda_{\xi^{\prime}}\right)(X+Y)+c\right] .
$$

In particular, if $\left[\xi^{\vee}\right] \uparrow \tau$ we have the factor $\lambda(X)+c$ for $\left(w, \xi^{\prime}\right)=(e, \xi)$. Moreover, for arbitrary $\left(w, \xi^{\prime}\right)$ the polynomial $\lambda \mapsto\left(w \lambda-w \Lambda_{\xi}+\Lambda_{\xi^{\prime}}\right)(X+Y)+c$ on $\mathfrak{a}_{\mathrm{qc}}^{*}$ is non-trivial because $X+Y \notin w\left(\mathfrak{j}_{0 \mathbf{c}}\right)$. All the assertions now easily follow.

Proof of Theorem 10.2. From (59) and (63) it follows that the polynomial $p_{0} \in \Pi\left(\mathfrak{a}_{\mathrm{q}}\right)$ has the property that for each $a \in A_{\mathrm{q}}^{+}(P)$ there exists a constant $C>0$ such that

$$
\left\|p_{0}(\lambda) \Phi_{P, w}(\lambda: a)\right\| \leq C(1+|\lambda|)^{\operatorname{deg} p_{0}} a^{\operatorname{Re} \lambda},
$$

for each $w \in \mathcal{W}$ and all $\lambda \in \overline{\mathfrak{a}}_{\mathrm{q}}^{*}(P, 0)$.

Let $D \in \mathbb{D}(G / H)$ be chosen according to Lemma 10.7 such that $p_{0} p$ divides $\mu(D: \tau)$. Then $D$ satisfies assertions (a) and (b) of the theorem. We shall prove that it also satisfies (c). Let $S$ and $\varphi$ be given as in (c), and put

$$
\tilde{\varphi}(\lambda):=\mu(D: \tau: \lambda) \varphi(\lambda) \quad\left(\lambda \in \mathfrak{a}_{\mathrm{q}}^{*}\right) .
$$

Notice that $\tilde{\varphi}$ belongs to the space $\mathcal{M}(S, 1)$. Indeed, from eqn. (38) it follows that $\tilde{\varphi}$ fulfills condition (a) of Definition 8.9, and since $p$ divides $\mu(D: \tau: \lambda)$ and $\mu(D: \tau: \lambda) \epsilon$ End $\left({ }^{\circ} \mathcal{C}(\tau)\right)$ commutes with the projection $\operatorname{pr}_{w}:{ }^{\circ} \mathcal{C}(\tau) \rightarrow{ }^{\circ} \mathcal{C}_{w}(\tau)$, for every $w \in \mathcal{W}$, conditions (b) and (c) hold as well. In fact, by the same argument (b) and (c) hold also for $p_{0}^{-1} \tilde{\varphi}$. 
Let now $w \in \mathcal{W}$. Combining estimate of Definition 8.9 (c) for $p_{0}^{-1} \tilde{\varphi}$ with (90) we see that for every $n \in \mathbb{N}$ and $a \in A_{\mathrm{q}}^{+}(P)$ there exists a constant $C>0$ such that

$$
\left\|\Phi_{P, w}(\lambda: a) \tilde{\varphi}_{w}(\lambda)\right\| \leq C(1+|\lambda|)^{-n} e^{h_{w S}(-\lambda)} a^{\operatorname{Re} \lambda}
$$

for all $\lambda \in \overline{\mathfrak{a}}_{\mathrm{q}}^{*}(P, 0)$. In particular, the function $\lambda \mapsto \Phi_{P, w}(\lambda: a) \tilde{\varphi}_{w}(\lambda)$ is holomorphic on an open neighborhood of $\overline{\mathfrak{a}}_{\mathrm{q}}^{*}(P, 0)$.

Now fix $a \in A_{\mathrm{q}}^{+}(P)$, and suppose that $\mathcal{J} \tilde{\varphi}(a w) \neq 0$. In view of the sphericality of $\mathcal{J} \tilde{\varphi}$ it suffices to show that

$$
\log a \in w S-c_{\mathrm{q}}^{+}(P) .
$$

Let $\eta \in \mathfrak{a}_{\mathbf{q}}^{*} \cap \mathfrak{a}_{\mathbf{q}}^{*}(P, 0)$ be fixed. Then $-\eta \in \mathfrak{a}_{\mathbf{q}}^{*}(\bar{P}, 0)$, and hence by Lemma 10.1 with $Q=P$ it suffices to show that

$$
h_{w S}(-\eta)+\eta(\log a) \geq 0 \text {. }
$$

By Theorem 7.5 and Definition 8.9, condition (a), we have

$$
\begin{aligned}
\mathcal{J} \tilde{\varphi}(a w) & =\int_{i \mathfrak{a}_{\mathrm{q}}^{*}} E^{\circ}(P: \tilde{\varphi}(\lambda): \lambda)(a w) d \lambda \\
& =\int_{i \mathfrak{a}_{\mathrm{q}}^{*}} \sum_{s \in W} \Phi_{P, w}(s \lambda: a)\left[C_{P \mid P}^{\circ}(s: \lambda) \tilde{\varphi}(\lambda)\right]_{w}(e) d \lambda \\
& =\int_{i \mathfrak{a}_{\mathrm{q}}^{*}} \sum_{s \in W} \Phi_{P, w}(s \lambda: a) \tilde{\varphi}_{w}(s \lambda)(e) d \lambda .
\end{aligned}
$$

From (91) we see that the integral and the sum in the last member may be interchanged, and since the measure $d \lambda$ on $i \mathfrak{a}_{\mathrm{q}}^{*}$ is $W$-invariant, we obtain:

$$
\mathcal{J} \tilde{\varphi}(a w)=|W| \int_{i \mathfrak{a}_{\mathrm{q}}^{*}} \Phi_{P, w}(\lambda: a) \tilde{\varphi}_{w}(\lambda)(e) d \lambda
$$

In view of (91) we obtain, by applying Cauchy's theorem:

$$
\mathcal{J} \tilde{\varphi}(a w)=|W| \int_{i \mathfrak{a}_{\mathrm{q}}^{*}} \Phi_{P, w}(\lambda+t \eta: a) \tilde{\varphi}_{w}(\lambda+t \eta)(e) d \lambda
$$

for all $t \geq 0$. From (93) and (91) it follows that

$$
\begin{aligned}
\|\mathcal{J} \tilde{\varphi}(a w)\| & \leq C_{1}|W| a^{t \eta} \int_{i \mathfrak{a}_{\mathrm{q}}^{*}}(1+|\lambda|)^{-n} d \lambda e^{t h_{w S}(-\eta)} \\
& =C_{2} e^{t\left[\eta(\log a)+h_{w S}(-\eta)\right]}(t \geq 0)
\end{aligned}
$$

with $C_{1}, C_{2}$ positive constants independent of $t$ (choose a suitable $n$ ). Since $\mathcal{J} \tilde{\varphi}(a w) \neq 0$, the last member of (94) cannot have limit equal to 0 as $t \rightarrow \infty$. Hence (92) follows and the proof is complete. 
Remark 10.9 Consider the special case where $G / H$ is a Riemannian symmetric space of the non-compact type. In this case the polynomial $p_{0}$ of (62) is 1 (see Remark 7.8). Furthermore, if we take $p=1$ in Definition 10.3, then by examination of the above proof we see that the operator $D \in \mathbb{D}_{p}$ in this case can be taken as the identity operator $D=1$. In this case the proof above is essentially Helgason's 'shift argument' (cf. [30], Ch. IV, Thm. 7.3).

\section{The behavior of supports}

In this section we combine the results of the previous three sections. We consider the composition $\mathcal{J F}$, which is a continuous linear map from $C_{c}^{\infty}(G / H: \tau)$ to $C^{\infty}(G / H: \tau)$; indeed this follows from Corollary 9.4 (a).

It is an immediate consequence of the results of the previous sections that there exists a differential operator $D \in \mathbb{D}(G / H)$ such that $\operatorname{det} \mu(D: \tau) \in \Pi\left(\mathfrak{a}_{\mathrm{q}}\right)$ and such that $D \mathcal{J} \mathcal{F}$ maps $C_{c}^{\infty}(G / H: \tau)$ continuously into itself. In fact, let $S$ be a $W_{K \cap H}$-invariant convex compact subset of $\mathfrak{a}_{\mathrm{q}}$. Then by Theorem 8.11, the Fourier transform $\mathcal{F}$ maps $C_{S}^{\infty}(G / H: \tau)$ continuously into $\mathcal{M}(S, \pi)$, where $\pi$ is the polynomial defined in (64). On the other hand, by Corollary 10.4 the subset $\mathbb{D}_{\pi} \subset \mathbb{D}(G / H)$ defined in Definition 10.3 (with $p=\pi$ ) is nonempty. Let $D \in \mathbb{D}_{\pi}$. Then, if in addition $S$ is $W$-invariant, Corollary 10.4 (and Remark $10.5)$ shows that $D \mathcal{J}$ maps $\mathcal{M}(S, \pi)$ continuously into $C_{S}^{\infty}(G / H: \tau)$. In particular, $D \mathcal{J} \mathcal{F}$ maps $C_{c}^{\infty}(G / H: \tau)$ continuously into itself. The purpose of the present section is to improve this result as follows.

Theorem 11.1 Let $D \in \mathbb{D}_{\pi}$. Then

$$
\operatorname{supp} D \mathcal{J F} f \subset \operatorname{supp} f \quad \text { for all } f \in C_{c}^{\infty}(G / H: \tau) \text {. }
$$

Proof. Let $D \in \mathbb{D}_{\pi}$. Then $D^{*}=D$ and it follows from Corollary 9.4 (b) that the linear endomorphism $D \mathcal{J} \mathcal{F}$ of $C_{c}^{\infty}(G / H: \tau)$ satisfies condition (a) of Proposition 11.2 below.

On the other hand, from Theorem 8.11 and condition (c) of Definition 10.3 (with $p=\pi$ ) it follows that $D \mathcal{J F}$ satisfies condition (b) of the proposition below. The theorem now follows from that proposition.

Proposition 11.2 Let $\mathcal{T}$ be a linear endomorphism of $C_{c}^{\infty}(G / H: \tau)$ such that the following conditions (a) and (b) are fulfilled:

(a) for all $f, g \in C_{c}^{\infty}(G / H: \tau)$ we have $\langle\mathcal{T} f \mid g\rangle=\langle f \mid \mathcal{T} g\rangle$;

(b) for every $W_{K \cap H}$-invariant compact convex subset $S \subset \mathfrak{a}_{\mathrm{q}}$, every $f \in C_{S}^{\infty}(G / H: \tau)$, and each $Q \in \mathcal{P}_{\sigma}^{\min }$ we have:

$$
\operatorname{supp} \mathcal{T} f \cap A_{\mathrm{q}}^{+}(Q) \subset \exp \left[S-c_{\mathrm{q}}^{+}(Q)\right]
$$


Then supp $\mathcal{T} f \subset \operatorname{supp} f$ for all $f \in C_{c}^{\infty}(G / H: \tau)$.

We first need a lemma. Recall from (66) the notion of $X_{S} \subset G / H$.

Lemma 11.3 Let $f \in C_{c}^{\infty}(G / H: \tau)$, and let $S \subset \mathfrak{a}_{\mathrm{q}}$ be a $W_{K \cap H}$-invariant open set. Assume that $\langle f \mid g\rangle=0$ for all $g \in C_{c}^{\infty}(G / H: \tau)$ with supp $g \subset X_{S}$. Then $f$ vanishes on $X_{S}$.

Proof. Let $Q \in \mathcal{P}_{\sigma}^{\min }$, and recall the definition of the map $T_{Q}^{\dagger}$ in (49). Then by (21) we have

$$
\int_{A_{\mathrm{q}}^{+}(Q)}\langle J(a) f(a) \mid h(a)\rangle d a=\left\langle f \mid T_{Q}^{\dagger} h\right\rangle=0
$$

for all $h \in C_{c}^{\infty}\left(A_{\mathrm{q}}^{+}(Q), V_{\tau}^{K \cap H_{\mathrm{M}}}\right)$ with $\operatorname{supp} h \subset \exp \left[S \cap \mathfrak{a}_{\mathrm{q}}^{+}(Q)\right]$. This implies that $J f$ and hence $f$ vanishes on $\exp \left[S \cap \mathfrak{a}_{\mathrm{q}}^{+}(Q)\right]$. Since the union of the chambers $\mathfrak{a}_{\mathrm{q}}^{+}(Q)$ is dense the lemma follows.

Proof of Proposition 11.2. If $S \subset \mathfrak{a}_{\mathrm{q}}$, then by $\mathrm{cl}(S)$, int $(S)$ we denote the closure and the interior of $S$ in $\mathfrak{a}_{\mathrm{q}}$, respectively.

Let $\mathfrak{S}$ denote the collection of all the closed $W_{K \cap H}$-invariant sets $S \subset \mathfrak{a}_{\mathrm{q}}$ with the property that for every $f \in C_{c}^{\infty}(G / H: \tau)$ we have:

$$
\operatorname{supp} f \cap A_{\mathrm{q}} \subset \exp S \Rightarrow \operatorname{supp} \mathcal{T} f \cap A_{\mathbf{q}} \subset \exp S .
$$

We shall establish the proposition by proving that $\mathfrak{S}$ contains all the closed $W_{K \cap H^{-}}$ invariant sets.

We start by discussing some set operations for members of $\mathfrak{S}$. If $f \in C_{c}^{\infty}(G / H: \tau)$, then by continuity of $f$ one readily verifies, for any open subset $U \subset \mathfrak{a}_{\mathrm{q}}$, that $f=0$ on $\exp U$ if and only if $f=0$ on $\exp [\operatorname{int}(\operatorname{cl}(U))]$. Passing to complements we see, for any closed subset $S \subset \mathfrak{a}_{\mathrm{q}}$, that supp $f \cap A_{\mathrm{q}} \subset \exp S \Longleftrightarrow \operatorname{supp} f \cap A_{\mathrm{q}} \subset \exp [\operatorname{cl}($ int $S)]$. From this we infer, for any $W_{K \cap H}$-invariant closed subset $S$ of $\mathfrak{a}_{\mathrm{q}}$, that

$$
S \in \mathfrak{S} \Longleftrightarrow \operatorname{cl}(\operatorname{int}(S)) \in \mathfrak{S}
$$

If $S \subset \mathfrak{a}_{\mathrm{q}}$ is a closed subset of $\mathfrak{a}_{\mathrm{q}}$, we put $S^{c}=\operatorname{cl}\left(\mathfrak{a}_{\mathrm{q}} \backslash S\right)=\mathfrak{a}_{\mathrm{q}} \backslash \operatorname{int}(S)$. We now observe that $S \in \mathfrak{S} \Rightarrow S^{c} \in \mathfrak{S}$. Indeed, let $S \in \mathfrak{S}$ and let $f \in C_{c}^{\infty}(G / H: \tau)$ with $\operatorname{supp} f \cap A_{\mathrm{q}} \subset \exp S^{c}$. By property (a) we then have $\langle\mathcal{T} f \mid g\rangle=\langle f \mid \mathcal{T} g\rangle=0$ for all $g \in C_{c}^{\infty}(G / H: \tau)$ with supp $g \cap A_{\mathrm{q}} \subset \exp [\operatorname{int}(S)]$. Hence $\mathcal{T} f$ vanishes on $\exp [\operatorname{int}(S)]$ by the lemma above, whence supp $\mathcal{T} f \cap A_{\mathrm{q}} \subset \exp S^{c}$.

It is clear that any intersection of sets from $\mathfrak{S}$ belongs to $\mathfrak{S}$. Moreover, if $\mathcal{S}$ is any collection of sets from $\mathfrak{S}$, then one readily checks that cl $\left[\cup_{S \in \mathcal{S}} \operatorname{int}(S)\right]$ equals $\left[\cap_{S \in \mathcal{S}} S^{c}\right]^{c}$, hence belongs to $\mathfrak{S}$.

From property (b) and Lemma 10.1 (b) we see that the closed $W$-invariant convex sets $S \subset \mathfrak{a}_{\mathbf{q}}$ belong to $\mathfrak{S}$. In particular the closed balls $\bar{B}(0, R), R>0$, are in $\mathfrak{S}$. Moreover, let $X \in \mathfrak{a}_{\mathrm{q}}$ be nonzero and let $r>0$; then the set

$$
\Xi_{X, r}=\left\{Y \in \mathfrak{a}_{\mathrm{q}} \mid\langle w X, Y\rangle \leq r\|X\| \quad \text { for all } \quad w \in W\right\}
$$


is $W$-invariant and convex, hence a member of $\mathfrak{S}$. We infer that the sets

$$
V_{\epsilon}=\bar{B}(0,\|X\|+\epsilon) \cap \Xi_{X,\|X\|-\epsilon}^{c}, \quad 0<\epsilon<\|X\|,
$$

also belong to $\mathfrak{S}$. Now if $Y \in V_{\epsilon}$ then $\|Y\| \leq\|X\|+\epsilon$ and $\langle w X, Y\rangle \geq(\|X\|-\epsilon)\|X\|$ for some $w \in W$, from which it follows that

$$
\|Y-w X\|^{2}=\|Y\|^{2}+\|X\|^{2}-2\langle w X, Y\rangle \leq 4\|X\| \epsilon
$$

so that $V_{\epsilon} \subset W \bar{B}(X, 4\|X\| \epsilon)$. On the other hand it is easily seen that $X$ lies in the interior of $V_{\epsilon}$, and thus we see that there is a basis of neighborhoods $U$ of $X$ satisfying $W U \in \mathfrak{S}$. Taking unions we conclude, for any closed $W$-invariant set $S \subset \mathfrak{a}_{\mathrm{q}}$, that the closure of its interior belongs to $\mathfrak{S}$, hence $S \in \mathfrak{S}$.

As before, let $X \in \mathfrak{a}_{\mathrm{q}}$ be nonzero and let $0<\epsilon<\|X\|$. Put $U=\bar{B}(X, \epsilon)$ and let $f \in C_{c}^{\infty}(G / H: \tau)$ with $\operatorname{supp} f \cap A_{\mathrm{q}} \subset \exp \left(W_{K \cap H} U\right)$, then since $W U \in \mathfrak{S}$ we have $\operatorname{supp} \mathcal{T} f \cap A_{\mathrm{q}} \subset \exp (W U)$. We claim that if $\epsilon$ is sufficiently small then actually supp $\mathcal{T} f \cap$ $A_{\mathrm{q}} \subset \exp \left(W_{K \cap H} U\right)$, so that $W_{K \cap H} U \in \mathfrak{S}$.

To establish the claim, let $Q \in \mathcal{P}_{\sigma}^{\min }$ and let $Y \in W U \cap \mathfrak{a}_{\mathrm{q}}^{+}(Q)$ be such that $\exp Y \in$ $\operatorname{supp} \mathcal{T} f$. Then

$$
Y \in \operatorname{conv} W_{K \cap H} U-c_{\mathrm{q}}^{+}(Q)
$$

by (b). Write $Y=Z-\gamma$ accordingly with $Z \in \operatorname{conv} W_{K \cap H} U$ and $\gamma \in c_{\mathrm{q}}^{+}(Q)$. Since $Y \in \mathfrak{a}_{\mathrm{q}}^{+}(Q)$ we have $\langle Y, \gamma\rangle \geq 0$, and it follows that $\|Z\|^{2} \geq\|Y\|^{2}+\|\gamma\|^{2}$. On the other hand, from $Z \in \operatorname{conv} W_{K \cap H} U$ we deduce that $\|Z\| \leq\|X\|+\epsilon$ and from $\|Y\| \in W U$ that $\|Y\| \geq\|X\|-\epsilon$. It follows that

$$
\|\gamma\|^{2} \leq\|Z\|^{2}-\|Y\|^{2} \leq(\|X\|+\epsilon)^{2}-(\|X\|-\epsilon)^{2}=4\|X\| \epsilon .
$$

We now observe that $W_{K \cap H} X$ is a finite subset of the sphere $S=\partial B(0,\|X\|)$, and hence conv $\left(W_{K \cap H} X\right) \cap S=W_{K \cap H} X$. From this we see: if $w \in W$ is such that $w X \notin W_{K \cap H} X$, then $w X \notin \operatorname{conv} W_{K \cap H} X$. Thus by choosing $\epsilon$ sufficiently small we may assume that the square of the distance from $w U$ to conv $W_{K \cap H} U$ exceeds $4\|X\| \epsilon$ for all $w \in W$ with $w X \notin W_{K \cap H} X$. Then (95) implies that $Y \in w U$ for some $w \in W$ with $w X \in W_{K \cap H} X$. But if $w X=s X$ for $w, s \in W$ then $w U=s U$, and hence we actually have $Y \in W_{K \cap H} U$. Thus for sufficiently small $\epsilon>0$ we have $\operatorname{supp} \mathcal{T} f \cap A_{\mathrm{q}}^{+}(Q) \subset \exp \left(W_{K \cap H} U\right)$ for every $Q \in \mathcal{P}_{\sigma}^{\min }$, and hence also $\operatorname{supp} \mathcal{T} f \cap A_{\mathrm{q}} \subset \exp \left(W_{K \cap H} U\right)$; this establishes the claim that $W_{K \cap H} \bar{B}(X, \epsilon) \in \mathfrak{S}$ when $\epsilon$ is sufficiently small. Taking unions we conclude, for any closed $W_{K \cap H}$-invariant set $S \subset \mathfrak{a}_{\mathrm{q}}$, that $\operatorname{cl}(\operatorname{int}(S)) \in \mathfrak{S}$, hence $S \in \mathfrak{S}$. This establishes the proposition.

\section{Asymptotic behavior of differential operators}

Let $Q \in \mathcal{P}_{\sigma}^{\min }$. We consider the algebra

$$
\mathcal{D}:=C^{\infty}\left(A_{\mathrm{q}}^{+}(Q)\right) \otimes \operatorname{End}\left(V_{\tau}^{K \cap H_{\mathrm{M}}}\right) \otimes S\left(\mathfrak{a}_{\mathrm{q}}\right)
$$


of differential operators on $A_{\mathrm{q}}^{+}(Q)$ with coefficients in $C^{\infty}\left(A_{\mathrm{q}}^{+}(Q)\right) \otimes \operatorname{End}\left(V_{\tau}^{K \cap H_{\mathrm{M}}}\right)$. The

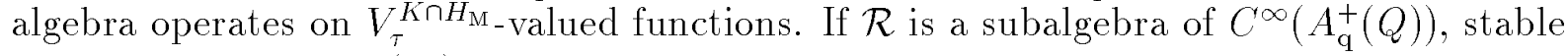
under the action of $S\left(\mathfrak{a}_{\mathrm{q}}\right)$ by differentiations, then

$$
\mathcal{D}_{\mathcal{R}}:=\mathcal{R} \otimes \operatorname{End}\left(V_{\tau}^{K \cap H_{\mathrm{M}}}\right) \otimes S\left(\mathfrak{a}_{\mathrm{q}}\right)
$$

is a subalgebra of $\mathcal{D}$. Via the map $u \mapsto 1 \otimes u$, the algebra $\operatorname{End}\left(V_{\tau}^{K \cap H_{\mathrm{M}}}\right) \otimes S\left(\mathfrak{a}_{\mathrm{q}}\right)$ will be identified with $\mathcal{D}_{\mathbb{C}}$, the subalgebra of constant coefficient operators in $\mathcal{D}$.

We recall that $\mathcal{O}=\mathcal{O}_{Q}$ is the ring of functions on $A_{\mathrm{q}}^{+}(Q)$ which may be expanded in series of the form (56). If $\mu \in \mathfrak{a}_{\mathrm{q} c}^{*}$, we write $e^{\mu}$ for the function $a \mapsto a^{\mu}$ on $A_{\mathrm{q}}$. Moreover, we denote by $\mathcal{O}^{+}=\mathcal{O}_{Q}^{+}$the ideal in $\mathcal{O}$ generated by the functions $e^{-\alpha}, \alpha \in \Delta(Q)$. If $P \in \mathcal{D}_{\mathcal{O}}$, then there exists a unique constant coefficient differential operator $\mathrm{C}(P)=$ $\mathrm{C}_{Q}(P) \in \operatorname{End}\left(V_{\tau}^{K \cap H_{\mathrm{M}}}\right) \otimes S\left(\mathfrak{a}_{\mathrm{q}}\right)$, called the constant part of $P$ along $A_{\mathbf{q}}^{+}(Q)$, such that

$$
P-\mathrm{C}(P) \in \mathcal{O}^{+} \otimes \operatorname{End}\left(V_{\tau}^{K \cap H_{\mathrm{M}}}\right) \otimes S\left(\mathfrak{a}_{\mathrm{q}}\right) .
$$

The following lemma is obvious.

Lemma 12.1 The map $P \mapsto \mathrm{C}(P), \mathcal{D}_{\mathcal{O}} \rightarrow \operatorname{End}\left(V_{\tau}^{K \cap H_{\mathrm{M}}}\right) \otimes S\left(\mathfrak{a}_{\mathrm{q}}\right)$ is a homomorphism of algebras.

From (54) we recall, for every $D \in \mathbb{D}(G / H)$, the definition of its $(Q, \tau)$-radial component $\Pi_{\tau}(D)=\Pi_{Q, \tau}(D)$. The map $D \mapsto \Pi_{\tau}(D)$ is a homomorphism of algebras from $\mathbb{D}(G / H)$ to $\mathcal{D}_{\mathcal{O}}$, by Lemma 7.3.

Recall also, from the text following formula (58), the definition of the algebra homomorphism $\mu(\cdot: \tau): \mathbb{D}(G / H) \rightarrow \operatorname{End}\left(V_{\tau}^{K \cap H_{\mathrm{M}}}\right) \otimes S\left(\mathfrak{a}_{\mathrm{q}}\right)$.

Put $\rho=\rho_{Q}$. Then the following generalization of [22], Lemma 26, describes the top order asymptotic behavior of the radial component $\Pi_{\tau}(D)$ of an operator $D \in \mathbb{D}(G / H)$.

Lemma 12.2 Let $D \in \mathbb{D}(G / H)$. Then $\Pi_{\tau}(D) \in \mathcal{D}_{\mathcal{O}}$, and

$$
\mathrm{C}\left(\Pi_{\tau}(D)\right)=e^{-\rho} \underline{\mu}(D: \tau) \circ e^{\rho} .
$$

Proof. From (13) we have

$$
D-\mathfrak{\prime}_{\bar{Q}}(D) \in \mathfrak{n}_{\bar{Q}} U(\mathfrak{g})+U(\mathfrak{g}) \mathfrak{h} .
$$

The result now follows from [4], Lemma 3.9, applied with the choice of positive roots $\Sigma^{+}=\Sigma(\bar{Q})$.

Thus the radial component $\Pi_{\tau}(D)$ of an operator $D \in \mathbb{D}(G / H)$ has a converging expansion on $A_{\mathrm{q}}^{+}(Q)$ :

$$
\Pi_{\tau}(D)=\sum_{\nu \in \mathbb{N} \Sigma(Q)} e^{-\nu} D_{\nu},
$$

with $D_{\nu} \in \operatorname{End}\left(V_{\tau}^{K \cap H_{\mathrm{M}}}\right) \otimes S\left(\mathfrak{a}_{\mathrm{q}}\right), D_{0}=e^{-\rho} \mu(D: \tau) \circ e^{\rho}$. In this paper we shall need such an expansion not only for the elements of the (commutative) algebra $\Pi_{\tau}(\mathbb{D}(G / H))$, but also for the elements in the commutant of this algebra in $\mathcal{D}$. The main result of this section is: 
Theorem 12.3 Let $P \in \mathcal{D}$ and assume that

$$
\left[P, \Pi_{\tau}(D)\right]=0 \quad(D \in \mathbb{D}(G / H)) .
$$

Then $P$ belongs to $\mathcal{D}_{\mathcal{O}}$; moreover $P$ is uniquely determined by $\mathrm{C}(P) \in \operatorname{End}\left(V_{\tau}^{K \cap H_{\mathrm{M}}}\right) \otimes$ $S\left(\mathfrak{a}_{\mathrm{q}}\right)$, its constant part along $A_{\mathbf{q}}^{+}(Q)$. Finally, if $P \neq 0$, then:

$$
\operatorname{order}(P-\mathrm{C}(P))<\operatorname{order} P \text {. }
$$

Remark 12.4 In the above formulation we have used the convention that the zero operator has order -1 . Notice that it follows from the above that $P$ and $\mathrm{C}(P)$ have the same order.

Remark 12.5 Theorem 12.3 is in fact valid under the weaker hypothesis that (99) holds for all $D \in \mathbb{Z}(G / H)=r(\mathfrak{Z})$. This follows from a rather straightforward modification of Lemma 12.10 and its proof.

Before discussing the proof of Theorem 12.3, we mention that it has the following consequence, in view of Lemma 12.2. Notice that the radial component of an operator $D \in \mathbb{D}(G / H)$ may have smaller order than $D$ itself (it may for example vanish for a non-trivial operator, cf. [12], Remark 2).

Corollary 12.6 Let $D \in \mathbb{D}(G / H), D \neq 0$. Then

$$
\operatorname{order}\left(\Pi_{\tau}(D)-e^{-\rho} \underline{\mu}(D: \tau) \circ e^{\rho}\right)<\operatorname{order} \Pi_{\tau}(D) \text {, }
$$

and $\Pi_{\tau}(D)$ is uniquely determined by $\underline{\mu}(D: \tau)$.

We first investigate the meaning of the commutation relations (99) in a somewhat more general situation. Let $W$ be a finite dimensional vector space, and let $\mathcal{D}$ be defined as in (96), but with $W$ in place of $V_{\tau}^{K \cap H_{\mathrm{M}}}$. Let $\mathcal{R}$ be a subalgebra of $C^{\infty}\left(A_{\mathrm{q}}^{+}(Q)\right)$, stable under the action of $S\left(\mathfrak{a}_{\mathrm{q}}\right)$ by differentiations, and define $\mathcal{D}_{\mathcal{R}}$ as in (97), again with $W$ in place of $V_{\tau}^{K \cap H_{\mathrm{M}}}$. Finally suppose that $\mathcal{B} \subset \mathcal{D}_{\mathcal{R}}$ is a subalgebra, and $E \subset \mathcal{D}_{\mathcal{R}}$ a finite dimensional linear subspace such that

$$
\mathcal{D}_{\mathcal{R}}=\mathcal{R} E \mathcal{B}
$$

where the expression in the right-hand side denotes the linear subspace spanned by all products of the form $f v b$ with $f \in \mathcal{R}, v \in E, b \in \mathcal{B}$.

Let $H_{1}, \ldots, H_{d}$ be a basis for $\mathfrak{a}_{\mathrm{q}}$. For a multi-index $\nu \in \mathbb{N}^{d}$, we write

$$
\partial^{\nu}=H_{1}^{\nu_{1}} \cdots H_{d}^{\nu_{d}}
$$


to emphasize that this element of $S\left(\mathfrak{a}_{\mathrm{q}}\right)$ will be viewed as a mixed partial derivative on $A_{\mathrm{q}}$. The $\partial^{\nu}, \nu \in \mathbb{N}^{d}$ constitute a basis for $S\left(\mathfrak{a}_{\mathbf{q}}\right)$. Given a differential operator $P \in \mathcal{D}$ we write

$$
P=\sum_{\nu} P_{\nu} \partial^{\nu}
$$

with coefficients $P_{\nu} \in C^{\infty}\left(A_{\mathrm{q}}^{+}(Q)\right) \otimes \operatorname{End}(W)$. The following lemma asserts that if $P$ commutes with $\mathcal{B}$, then the vector composed of its coefficients satisfies a cofinite system of differential equations.

Lemma 12.7 Let $P \in \mathcal{D}$ be a differential operator of order $n \in \mathbb{N}$, and assume that

$$
[P, D]=0 \quad(D \in \mathcal{B})
$$

Then there exists an $s \in \mathbb{N}$ and finitely many functions $\varphi_{i \alpha \nu}^{\mu \beta} \in \mathcal{R} \otimes \operatorname{End}(\operatorname{End}(W))$ $(1 \leq i \leq d,|\mu|,|\nu| \leq s,|\alpha|,|\beta| \leq n)$, such that for every $1 \leq i \leq d,|\mu| \leq s$ and all $\alpha$ with $|\alpha| \leq n$ we have:

$$
H_{i} \partial^{\mu}\left(P_{\alpha}\right)=\sum_{\substack{|\nu| \leq s \\|\beta| \leq n}} \varphi_{i \alpha \nu}^{\mu \beta}\left(\partial^{\nu}\left(P_{\beta}\right)\right),
$$

as an equation in $C^{\infty}\left(A_{\mathbf{q}}^{+}(Q)\right) \otimes \operatorname{End}(W)$.

Proof. By the assumption (100) there exists an $s \in \mathbb{N}$ such that

$$
\mathcal{D}_{\mathcal{R}}=\mathcal{R} \operatorname{End}(W) S_{s}\left(\mathfrak{a}_{\mathrm{q}}\right) \mathcal{B}
$$

where $S_{s}\left(\mathfrak{a}_{\mathrm{q}}\right)$ denotes the subspace of elements of order at most $s$ in $S\left(\mathfrak{a}_{\mathrm{q}}\right)$. For $1 \leq i \leq d$ and $|\mu| \leq s$ we have

$$
\left[H_{i} \partial^{\mu}, P\right]=\sum_{\beta}\left[H_{i} \partial^{\mu}, P_{\beta}\right] \partial^{\beta},
$$

and by the Leibniz rule there exist constants $c_{i \beta \gamma \nu}^{\mu} \in \mathbb{C}$, such that

$$
\left[H_{i} \partial^{\mu}, P_{\beta}\right]=H_{i} \partial^{\mu}\left(P_{\beta}\right)+\sum_{\substack{\gamma \\|\nu| \leq s}} c_{i \beta \gamma \nu}^{\mu} \partial^{\nu}\left(P_{\beta}\right) \partial^{\nu}
$$

On the other hand, from (103) we see that $H_{i} \partial^{\mu}$ is a finite sum of elements of the form $f A \partial^{\lambda} B$, with $f \in \mathcal{R}, A \in \operatorname{End}(W), B \in \mathcal{B}$, and $|\lambda| \leq s$. Moreover, $\left[f A \partial^{\lambda} B, P\right]=$ $\left[f A \partial^{\lambda}, P\right] B$ by the hypothesis (101). The latter expression is a finite sum of terms of the form $\psi_{\gamma \nu}^{\beta}\left(\partial^{\nu}\left(P_{\beta}\right)\right) \partial^{\gamma}$, with $\psi_{\gamma \nu}^{\beta} \in \mathcal{R} \otimes \operatorname{End}(\operatorname{End}(W))$ and $|\nu| \leq s$. From this reasoning we conclude that

$$
\left[H_{i} \partial^{\mu}, P\right]=\sum_{\substack{\gamma, \beta \\|\nu| \leq s}} \phi_{i \gamma \nu}^{\mu \beta}\left(\partial^{\nu}\left(P_{\beta}\right)\right) \partial^{\gamma},
$$

with $\phi_{i \gamma \nu}^{\mu \beta} \in \mathcal{R} \otimes \operatorname{End}(\operatorname{End}(W))$. Substituting (105) in the right-hand side of (104) and comparing the resulting coefficient of $\partial^{\alpha}$ with the corresponding coefficient of $\partial^{\alpha}$ in the right-hand side of (106), we obtain (102), with $\varphi_{i \alpha \nu}^{\mu \beta}=\phi_{i \alpha \nu}^{\mu \beta}-c_{i \beta[\alpha-\beta] \nu}^{\mu}$. 
We will apply the above lemma in two special cases. Let $\mathbf{E}\left(A_{\mathbf{q}}\right)$ denote the space of exponential polynomial functions on $A_{\mathrm{q}}$, i.e. the space of finite linear combinations of functions of the form $a \mapsto(\log a)^{m} a^{\lambda}$, with $\lambda \in \mathfrak{a}_{\mathrm{qc}}^{*}, m \in \mathbb{N}^{d}$. Here we have used the notation

$$
(\log a)^{m}:=\prod_{j=1}^{d}\left[H_{j}^{*}(\log a)\right]^{m_{j}},
$$

with $H_{1}^{*}, \ldots, H_{d}^{*}$ the basis of $\mathfrak{a}_{\mathrm{q}}^{*}$ dual to $H_{1}, \ldots, H_{d}$. A subalgebra $\mathcal{A}_{1}$ of a commutative algebra $\mathcal{A}_{2}$ will be called cofinite if $\mathcal{A}_{2}$ is finitely generated as a $\mathcal{A}_{1}$-module.

Lemma 12.8 If $\mathcal{A} \subset \mathbb{D}(G / H)$ is a cofinite subalgebra, then the algebra $\operatorname{End}\left(V_{\tau}^{K \cap H_{\mathrm{M}}}\right) \otimes$ $S\left(\mathfrak{a}_{\mathrm{q}}\right)$ is a finite $\underline{\mu}(\mathcal{A}: \tau)$-module from the right (as well as from the left).

Proof. In view of the isomorphism (16) this is a straightforward consequence of the fact that the algebra $\mathbb{D}\left(M_{1} / H_{\mathrm{M}_{1}}\right)$ is finite as a $\mu(\mathbb{D}(G / H))$-module, cf. [7], eqn. (27).

Lemma 12.9 Let $\mathcal{A} \subset \mathbb{D}(G / H)$ be a cofinite subalgebra and let $P \in \mathcal{D}$ be an operator such that $[P, \mu(D: \tau)]=0$ for every $D \in \mathcal{A}$. Then $P$ has coefficients of exponential polynomial type, i.e. its coefficients belong to $\mathbf{E}\left(A_{\mathrm{q}}\right) \otimes \operatorname{End}\left(V_{\tau}^{K \cap H_{\mathrm{M}}}\right)$.

Proof. In view of the previous lemma, we may apply Lemma 12.7 with $W=V_{\tau}^{K \cap H_{\mathrm{M}}}$, $\mathcal{R}=\mathbb{C}$, and $\mathcal{B}=\mu(\mathcal{A}: \tau)$, and infer that the coefficients of $P$ satisfy equations of the form (102). Let $\mathcal{N}$ be the set of pairs $(\nu, \alpha) \in \mathbb{N}^{d} \times \mathbb{N}^{d}$, with $|\nu| \leq s$ and $|\alpha| \leq$ order $P$, and define the $\mathbb{C}^{\mathcal{N}} \otimes \operatorname{End}\left(V_{\tau}^{K \cap H_{\mathrm{M}}}\right)$-valued function $\mathbf{P}$ on $A_{\mathbf{q}}^{+}(Q)$ by

$$
\mathbf{P}_{(\nu, \alpha)}(a)=\partial^{\nu} P_{\alpha}(a) \quad((\nu, \alpha) \in \mathcal{N}) .
$$

Then we may rewrite the above system of equations in vector form:

$$
H_{i} \mathbf{P}=M_{i} \mathbf{P} \quad(1 \leq i \leq d)
$$

where $M_{i} \in \operatorname{End}\left(\mathbb{C}^{\mathcal{N}} \otimes \operatorname{End}\left(V_{\tau}^{K \cap H_{\mathrm{M}}}\right)\right)$. Let $M: \mathfrak{a}_{\mathrm{q}} \rightarrow \operatorname{End}\left(\mathbb{C}^{\mathcal{N}} \otimes \operatorname{End}\left(V_{\tau}^{K \cap H_{\mathrm{M}}}\right)\right)$ be the linear map determined by $M\left(H_{i}\right)=M_{i}$. Then we infer that

$$
\mathbf{P}(a)=e^{M(\log a)} C \quad\left(a \in A_{\mathrm{q}}^{+}(Q)\right),
$$

for a suitable $C \in \mathbb{C}^{\mathcal{N}} \otimes \operatorname{End}\left(V_{\tau}^{K \cap H_{\mathrm{M}}}\right)$. It follows that the coefficient $P_{\alpha}=\partial^{0} P_{\alpha}$ of $\mathbf{P}$ is of exponential polynomial type.

If $\mathcal{A}=\mathbb{D}(G / H)$ then we can refine the above lemma, and we obtain the following 'flat' version of Theorem 12.3.

Proposition 12.10 Let $P \in \mathcal{D}$, and assume that

$$
[P, \underline{\mu}(D: \tau)]=0 \quad(D \in \mathbb{D}(G / H)) .
$$


Then $P$ has constant coefficients, i.e. it belongs to $\operatorname{End}\left(V_{\tau}^{K \cap H_{\mathrm{M}}}\right) \otimes S\left(\mathfrak{a}_{\mathrm{q}}\right)$.

Proof. By the previous lemma, the coefficients of $P$ are of exponential polynomial type. Let $S \subset \mathfrak{a}_{\mathrm{qc}}^{*}$ be the finite set of exponents occurring in these coefficients. Then we may write $P$ as a finite sum

$$
P(a)=\sum_{\substack{\xi \in S \\ m \in \mathbb{N}^{d}}}(\log a)^{m} a^{\xi} P_{\xi, m},
$$

with $P_{\xi, m} \in \operatorname{End}\left(V_{\tau}^{K \cap H_{\mathrm{M}}}\right) \otimes S\left(\mathfrak{a}_{\mathrm{q}}\right)$. Put $P_{\xi}(a)=a^{\xi} \sum_{m}(\log a)^{m} P_{\xi, m}$, then one easily sees that every $P_{\xi}$ commutes with all $\mu(D: \tau), D \in \mathbb{D}(G / H)$. Thus we may as well assume that $S$ consists of a single element $\xi$.

For $\lambda \in \mathfrak{a}_{\mathrm{qc}}^{*}$ and $v \in V_{\tau}^{K \cap H_{\mathrm{M}}}$, define the function $e^{\lambda} v: A_{\mathrm{q}} \rightarrow V_{\tau}^{K \cap H_{\mathrm{M}}}, a \mapsto a^{\lambda} v$. Moreover, let $\mathcal{E}_{\lambda}$ denote the following finite dimensional linear span of functions

$$
\mathcal{E}_{\lambda}=\left\langle e^{s \lambda} v \mid s \in W, v \in V_{\tau}^{K \cap H_{\mathrm{M}}}\right\rangle .
$$

In view of the lemma below, for generic $\lambda$ the space $\mathcal{E}_{\lambda}$ is a sum of joint eigenspaces for $\underline{\mu}(\mathbb{D}(G / H): \tau)$. This implies that $P$ maps $\mathcal{E}_{\lambda}$ into itself. In particular the function

$$
P\left(e^{\lambda} v\right)(a)=a^{\xi+\lambda} \sum_{m}(\log a)^{m} P_{\xi, m}(\lambda) v
$$

is contained in $\mathcal{E}_{\lambda}$ for generic $\lambda \in \mathfrak{a}_{\mathrm{qc}}^{*}$. Now $\xi+\lambda \notin W \lambda \backslash\{\lambda\}$ for generic $\lambda$, and we conclude that we must have $\xi=0$, and $P_{\xi, m}(\lambda)=0$ for $m \neq 0$. Hence $P$ has constant coefficients.

Lemma 12.11 For $\lambda$ in the complement of a locally finite union of hyperplanes in $\mathfrak{a}_{\mathrm{qc}}^{*}$ the space $\mathcal{E}_{\lambda}$ defined in (108) is a sum of simultaneous eigenspaces for $\underline{\mu}(\mathbb{D}(G / H): \tau)$.

Proof. Let $\mathfrak{b}$ be a Cartan subspace of $\mathfrak{q}$, containing $\mathfrak{a}_{\mathrm{q}}$, and let $\gamma: \mathbb{D}(G / H) \rightarrow I(\mathfrak{b})$ be the associated Harish-Chandra isomorphism.

If $\lambda \in \mathfrak{a}_{\mathbf{q}}^{*}$, we define $\mathcal{E}_{\lambda}^{\prime}$ to be the space of functions $f \in C^{\infty}\left(A_{\mathbf{q}}\right) \otimes V_{\tau}^{K \cap H_{\mathrm{M}}}$ satisfying the system of differential equations:

$$
\underline{\mu}(D: \tau) f=\underline{\mu}(D: \tau: \lambda) f \quad(D \in \mathbb{D}(G / H)) .
$$

Then obviously $e^{\lambda} v \in \mathcal{E}_{\lambda}^{\prime}$ for all $v \in V_{\tau}^{K \cap H_{\mathrm{M}}}$ and hence

$$
\mathcal{E}_{\lambda} \subset \sum_{s \in W} \mathcal{E}_{s \lambda}^{\prime}
$$

By (58) and (39) the endomorphism $\underline{\mu}(D: \tau: \lambda)$ of $V_{\tau}^{K \cap H_{\mathrm{M}}}$ is semisimple with eigenvalues of the form $\gamma(D: \Lambda+\lambda), \Lambda \in L$, for all $D \in \mathbb{D}(G / H), \lambda \in \mathfrak{a}_{\mathrm{q} c}^{*}$. Let $T=\cup_{w \in N_{K}\left(\mathfrak{a}_{\mathrm{q}}\right)} w L$, then it follows from the Weyl-invariance of $\gamma(D)$ that $\mu(D: \tau: s \lambda)$ has eigenvalues of the form $\gamma(D: \Lambda+\lambda)$ with $\Lambda \in T$, for all $s \in W$. For $\Lambda \in \bar{T}$, let $\mathcal{E}_{\lambda, \Lambda}^{\prime}$ be the space of functions $f \in C^{\infty}\left(A_{\mathrm{q}}\right) \otimes V_{\tau}^{K \cap H_{\mathrm{M}}}$ such that

$$
\underline{\mu}(D: \tau) f=\gamma(D: \Lambda+\lambda) f \quad(D \in \mathbb{D}(G / H)) .
$$


Then it follows from the above discussion that

$$
\mathcal{E}_{s \lambda}^{\prime} \subset \sum_{\Lambda \in T} \mathcal{E}_{\lambda, \Lambda}^{\prime}
$$

for all $s \in W$.

On the other hand, in view of (58) it follows from [7], Prop. 13.5, that $\mathcal{E}_{\lambda, \Lambda}^{\prime} \subset \mathcal{E}_{\lambda}$, for $\lambda$ in the complement of a locally finite union of hyperplanes. Together with (110) and (111) this completes the proof.

Proposition 12.12 Let $P \in \mathcal{D}$, and let $\mathcal{A} \subset \mathbb{D}(G / H)$ be a cofinite subalgebra. Assume that

$$
\left[P, \Pi_{\tau}(D)\right]=0 \quad(D \in \mathcal{A}) .
$$

Then $P$ belongs to $\mathbf{E}\left(A_{\mathrm{q}}\right) \mathcal{O} \otimes \operatorname{End}\left(V_{\tau}^{K \cap H_{\mathrm{M}}}\right) \otimes S\left(\mathfrak{a}_{\mathrm{q}}\right)$.

Proof. It follows from [5], Lemma 3.8, that there exists a finite dimensional subspace $E^{\prime} \subset \operatorname{End}\left(V_{\tau}^{K \cap H_{\mathrm{M}}}\right) \otimes S\left(\mathfrak{a}_{\mathrm{q}}\right)$ such that

$$
\mathcal{D}_{\mathcal{O}}=\mathcal{O} E^{\prime} \Pi_{\tau}(\mathbb{Z}(G / H))
$$

The above equality certainly holds with $\mathbb{Z}(G / H)$ replaced by $\mathbb{D}(G / H)$, and since $\mathbb{D}(G / H)$ is a finite module over $\mathcal{A}$, it also holds with $\mathbb{Z}(G / H)$ and $E^{\prime}$ replaced by $\mathcal{A}$ and a finite dimensional linear subspace $E$ of $\mathcal{D}_{\mathcal{O}}$ respectively. Therefore we may apply Lemma 12.7 with $\mathcal{R}=\mathcal{O}$ and $\mathcal{B}=\Pi_{\tau}(\mathcal{A})$. It follows that the coefficients of $P$ satisfy equations of the form (102). As in the proof of Lemma 12.7 these equations may be rewritten in the vector form (107), but this time with $M_{i} \in \mathcal{O} \otimes \operatorname{End}\left(\mathbb{C}^{\mathcal{N}} \otimes \operatorname{End}\left(V_{\tau}^{K \cap H_{\mathrm{M}}}\right)\right)$. The conclusion now follows from [17], App. 1, if one proceeds along the lines of [4], pp. 233-234.

Proof of Theorem 12.3. Let $P$ satisfy the hypotheses of the theorem. Then by the previous proposition, $P$ has an absolutely converging series expansion:

$$
P(a)=\sum_{\substack{\xi \in X \\ m \in M}}(\log a)^{m} a^{\xi} P_{\xi, m} \quad\left(a \in A_{\mathrm{q}}^{+}(Q)\right)
$$

Here $M \subset \mathbb{N}^{d}$ is a finite subset, and $X$ is a subset of $\mathfrak{a}_{\mathrm{q} c}^{*}$ of the form $X=S+(-\mathbb{N} \Sigma(Q))$, with $S \subset \mathfrak{a}_{\mathrm{qc}}^{*}$ finite. Moreover, $P_{\xi, m} \in \operatorname{End}\left(V_{\tau}^{K \cap H_{\mathrm{M}}}\right) \otimes S\left(\mathfrak{a}_{\mathrm{q}}\right)$. Here it is possible that $S=\emptyset$; this means that $X=\emptyset$ and $P=0$. An element $\xi \in X$ for which there exists an $m \in M$ such that $P_{\xi, m} \neq 0$ will be called an exponent of $P$ (along $Q$ ). The maximal elements in the set of exponents with respect to the ordering $\preceq$ on $\mathfrak{a}_{\mathrm{qc}}^{*}$ (defined by $\xi_{1} \preceq$ $\left.\xi_{2} \Longleftrightarrow \xi_{2}-\xi_{1} \in \mathbb{N} \Sigma(Q)\right)$ are called the leading exponents of $P$. We denote the set of these leading exponents by $T$ and define the associated leading part of $P$ to be the operator

$$
P_{\mathrm{L}}(a):=\sum_{\substack{s \in T \\ m \in M}}(\log a)^{m} a^{s} P_{s, m}
$$


By construction we have $P_{\mathrm{L}}=0 \Rightarrow P=0$. We now consider the commutation relations (99) once more. Inserting the expansions (112) and (98) of $P$ and $\Pi_{\tau}(D)$, we conclude that $\left[P_{\mathrm{L}}, e^{-\rho} \underline{\mu}(D: \tau) \circ e^{\rho}\right]=0$, or, equivalently,

$$
\left[e^{\rho} P_{\mathrm{L}} \circ e^{-\rho}, \underline{\mu}(D: \tau)\right]=0 \quad(D \in \mathbb{D}(G / H)) .
$$

Then by Lemma 12.10 we infer that $e^{\rho} P_{\mathrm{L}} \circ e^{-\rho}$, and hence $P_{\mathrm{L}}$, has constant coefficients. Thus we see that $T \subset\{0\}$, and that $P_{0, m}=0$ for $m \neq 0$. Hence

$$
P(a)=\sum_{m \in M}(\log a)^{m} \sum_{\nu \in \mathbb{N} \Sigma(Q)} a^{-\nu} P_{-\nu, m},
$$

with $P_{0, m}=0$ for $m \neq 0$. In particular we see that $P_{\mathrm{L}}=P_{0,0}$. Hence the map $P \mapsto P_{0,0}$ is an injective linear map from the commutant of $\Pi_{\tau}(\mathbb{D}(G / H))$ into $\operatorname{End}\left(V_{\tau}^{K \cap H_{\mathrm{M}}}\right) \otimes S\left(\mathfrak{a}_{\mathrm{q}}\right)$. Inserting the expansion (113) together with that of $\Pi_{\tau}(D)$ once more in (99), we now find, for every $D \in \mathbb{D}(G / H)$ :

$$
\begin{aligned}
0 & =\left[P, \Pi_{\tau}(D)\right](a) \\
& =\sum_{\substack{m \in M \\
\nu, \mu \in \mathbb{N} \Sigma(Q)}}\left[(\log a)^{m} a^{-\nu} P_{-\nu, m}, a^{-\mu} D_{\mu}\right] \\
& =\sum_{\substack{m \in M \\
\nu, \mu \in \mathbb{N} \Sigma(Q)}}\left((\log a)^{m}\left[a^{-\nu} P_{-\nu, m}, a^{-\mu} D_{\mu}\right]+a^{-\mu}\left[(\log a)^{m}, D_{\mu}\right] a^{-\nu} P_{-\nu, m}\right) .
\end{aligned}
$$

Shrinking $M$ if necessary, we may assume that for each $m \in M$ at least one of the $P_{-\nu, m}$ $(\nu \in \mathbb{N} \Sigma(Q))$ is non-trivial. Suppose now that $M \neq \emptyset$ and that $m_{0}$ is an element in $M$ of maximal length $|m|$. Then, since $\left[(\log a)^{m_{0}}, D_{\mu}\right]$ has powers of $\log a$ of length strictly smaller than $m_{0}$, it follows from the above equations that:

$$
\sum_{\nu, \mu \in \mathbb{N} \Sigma(Q)}\left[a^{-\nu} P_{-\nu, m_{0}}, a^{-\mu} D_{\mu}\right]=0,
$$

that is, the differential operator

$$
P_{m_{0}}(a)=\sum_{\nu \in \mathbb{N} \Sigma(Q)} a^{-\nu} P_{-\nu, m_{0}}
$$

also commutes with $\Pi_{\tau}(D)$, for every $D \in \mathbb{D}(G / H)$. By the first part of the proof this forces $P_{0, m_{0}} \neq 0$, which in turn forces $m_{0}=0$. Hence $M \subset\{0\}$, and writing $P_{\nu}=P_{-\nu, 0}$ we obtain:

$$
P(a)=\sum_{\nu \in \mathbb{N} \Sigma(Q)} a^{-\nu} P_{\nu} \quad\left(a \in A_{\mathrm{q}}^{+}(Q)\right) .
$$

Hence $P \in \mathcal{O} \otimes \operatorname{End}\left(V_{\tau}^{K \cap H_{\mathrm{M}}}\right) \otimes S\left(\mathfrak{a}_{\mathrm{q}}\right)$, as claimed in the theorem. Moreover, $\mathrm{C}(P)=P_{0,0}$ determines $P$ uniquely. Finally, if $P \neq 0$, it remains to prove the statement on the orders. This is done exactly as in [12], proof of Prop. 1, by means of a recursion formula for the $P_{\nu}$, arising from the commutation of $P$ with the Laplacian. 
We conclude this section by discussing some properties of the constant part of an operator $P \in \mathcal{D}_{\mathcal{O}}$ which will be useful at a later stage. The following lemma is easy to prove.

Lemma 12.13 Let $\nu \in \mathfrak{a}_{\mathrm{qc}}^{*}$. Then conjugation by the function $e^{\nu}: a \mapsto a^{\nu}$ leaves the algebra $\mathcal{D}_{\mathcal{O}}$ as well as its subalgebra $\mathcal{D}_{\mathbb{C}}$ invariant, and defines automorphisms of these algebras. Moreover, for every $P \in \mathcal{D}_{\mathcal{O}}$ we have:

$$
\mathrm{C}\left(e^{\nu} P \circ e^{-\nu}\right)=e^{\nu} \mathrm{C}(P) \circ e^{-\nu} .
$$

Lemma 12.14 Conjugation by the function $a \mapsto J(a)^{1 / 2}$ leaves the algebra $\mathcal{D}_{\mathcal{O}}$ invariant, and defines an automorphism $P \mapsto \widetilde{P}$ of $\mathcal{D}_{\mathcal{O}}$. Moreover, if $P \in \mathcal{D}_{\mathcal{O}}$, then

$$
\mathrm{C}(\widetilde{P})=e^{\rho} \mathrm{C}(P) \circ e^{-\rho} \text {. }
$$

Proof. Observe that $J(a)^{1 / 2}=a^{\rho} \varphi$, with $\varphi \in \mathcal{O}$ an invertible element such that $\varphi(a) \rightarrow 1$ if $a \stackrel{Q}{\longrightarrow} \infty$ (i.e. if $a^{-\alpha} \rightarrow 0$ for each $\alpha \in \Sigma(Q)$ ). Using the previous lemma we find that

$$
\mathrm{C}\left(J^{1 / 2} P \circ J^{-1 / 2}\right)=e^{\rho} \mathrm{C}\left(\varphi P \circ \varphi^{-1}\right) \circ e^{-\rho}=e^{\rho} \mathrm{C}(P) \circ e^{-\rho} .
$$

Let $\varphi \in C_{c}^{\infty}\left(\mathfrak{a}_{\mathrm{q}}^{+}(Q)\right)$ be fixed such that

$$
\int_{\mathfrak{a}_{\mathrm{q}}^{+}(Q)}|\varphi(X)|^{2} d X=1
$$

where $d X$ denotes the Lebesgue measure on $\mathfrak{a}_{\mathrm{q}}$ which via exp corresponds to the invariant measure $d a$ on $A_{\mathrm{q}}$. Let $d:=\operatorname{dim} \mathfrak{a}_{\mathrm{q}}$. Then for $v \in V_{\tau}^{K \cap H_{\mathrm{M}}}, \nu \in \mathfrak{a}_{\mathrm{qc}}^{*}$ and $\epsilon>0$ we define the function $f_{\epsilon, \nu, v} \in C_{c}^{\infty}\left(A_{\mathrm{q}}^{+}(Q)\right) \otimes V_{\tau}^{K \cap H_{\mathrm{M}}}$ by

$$
f_{\epsilon, \nu, v}(\exp X)=\epsilon^{d / 2} e^{\nu(X)} J(\exp X)^{-1 / 2} \varphi(\epsilon X) v \text {. }
$$

Then supp $f_{\epsilon, \nu, v}$ tends to infinity in $A_{\mathrm{q}}^{+}(Q)$ as $\epsilon \searrow 0$, and using (53) and the relation (50) one readily checks that

$$
\left\langle T_{Q}^{\dagger} f_{\epsilon, \nu, v} \mid T_{Q}^{\dagger} f_{\epsilon,-\bar{\nu}, w}\right\rangle=\left\langle f_{\epsilon, \nu, v} \mid f_{\epsilon,-\bar{\nu}, w}\right\rangle_{J}=\langle v \mid w\rangle
$$

Lemma 12.15 Let $P \in \mathcal{D}_{\mathcal{O}}$. Then for $v, w \in V_{\tau}^{K \cap H_{\mathrm{M}}}$ and $\nu \in \mathfrak{a}_{\mathrm{q}}^{*}$ we have

$$
\lim _{\epsilon \backslash 0}\left\langle P f_{\epsilon, \nu, v} \mid f_{\epsilon,-\bar{\nu}, w}\right\rangle_{J}=\langle\mathrm{C}(\tilde{P})(\nu) v \mid w\rangle
$$

Before proving the lemma we mention the following easy estimate for functions in $\mathcal{O}^{+}$. 
Lemma 12.16 Let $\psi \in \mathcal{O}^{+}$, and let $\Omega \subset \mathfrak{a}_{\mathrm{q}}^{+}(Q)$ be compact. Then there exist constants $r, C>0$ such that

$$
|\psi(\exp t X)| \leq C e^{-r t} \quad(X \in \Omega, t \geq 1)
$$

Proof. This follows easily from the observations in Remark 7.2.

Proof of Lemma 12.15. Let $k$ be the order of $P$, let $S_{k}\left(\mathfrak{a}_{\mathrm{q}}\right)$ denote the subspace of elements of order at most $k$ in $S\left(\mathfrak{a}_{\mathrm{q}}\right)$, and fix a norm $|\cdot|$ on $\operatorname{End}\left(V_{\tau}^{K \cap H_{\mathrm{M}}}\right) \otimes S_{k}\left(\mathfrak{a}_{\mathrm{q}}\right)$.

For $\epsilon>0, v \in V_{\tau}^{K \cap H_{\mathrm{M}}}$, put $\varphi_{\epsilon}(a)=\varphi(\epsilon \log a)$, and define $g_{\epsilon, v}: A_{\mathrm{q}} \rightarrow V_{\tau}^{K \cap H_{\mathrm{M}}}$ by $g_{\epsilon, v}(a)=\epsilon^{d / 2} \varphi_{\epsilon}(a) v$. Here we have extended $\varphi$ by zero to a compactly supported function on $\mathfrak{a}_{\mathrm{q}}$. For all $\epsilon>0$ we have:

$$
\left\langle g_{\epsilon, v} \mid g_{\epsilon, w}\right\rangle:=\int_{A_{\mathrm{q}}}\left\langle g_{\epsilon, v}(a) \mid g_{\epsilon, w}(a)\right\rangle_{\tau} d a=\langle v \mid w\rangle_{\tau} \quad\left(v, w \in V_{\tau}^{K \cap H_{\mathrm{M}}}\right) .
$$

We will first investigate the behavior of $\left\langle P g_{\epsilon, v} \mid g_{\epsilon, w}\right\rangle$. One readily verifies that there exists a constant $C>0$, only depending on $\varphi$, such that for every $U \in \operatorname{End}\left(V_{\tau}^{K \cap H_{\mathrm{M}}}\right) \otimes S_{k}\left(\mathfrak{a}_{\mathrm{q}}\right)$, and all $\epsilon \in] 0,1], v \in V_{\tau}^{K \cap H_{\mathrm{M}}}$ one has

$$
\left|U g_{\epsilon, v}(a)-U(0) g_{\epsilon, v}(a)\right| \leq \epsilon^{1+d / 2} C|U||v|
$$

and

$$
\left|U g_{\epsilon, v}(a)\right| \leq C \epsilon^{d / 2}|U||v|
$$

for all $a \in A_{\mathrm{q}}$. Using the estimate (119) and Lemma 12.16 we see that for $P_{+}:=P-$ $\mathrm{C}(P) \in \mathcal{O}^{+} \otimes \operatorname{End}\left(V_{\tau}^{K \cap H_{\mathrm{M}}}\right) \otimes S_{k}\left(\mathfrak{a}_{\mathrm{q}}\right)$ there exists a constant $r>0$ such that

$$
\left\langle P_{+} g_{\epsilon, v}(a) \mid g_{\epsilon, w}(a)\right\rangle=\mathrm{O}\left(e^{-r / \epsilon}\right) \quad(\epsilon \searrow 0)
$$

uniformly in $a \in A_{\mathrm{q}}$. Combining this estimate with (118) for $U=\mathrm{C}(P)$ and with (119), and integrating over $A_{\mathrm{q}}$, we find that

$$
\left\langle[P-\mathrm{C}(P)(0)] g_{\epsilon, v} \mid g_{\epsilon, w}\right\rangle=\mathrm{O}\left(\epsilon^{d+1} \operatorname{vol}\left(\operatorname{supp} \varphi_{\epsilon}\right)\right)=\mathrm{O}(\epsilon)
$$

But

$$
\left\langle\mathrm{C}(P)(0) g_{\epsilon, v} \mid g_{\epsilon, w}\right\rangle=\left\langle g_{\epsilon, \mathrm{C}(P)(0) v} \mid g_{\epsilon, w}\right\rangle=\langle\mathrm{C}(P)(0) v \mid w\rangle,
$$

for all $\epsilon>0$, by (117). It follows that

$$
\lim _{\epsilon \backslash 0}\left\langle P g_{\epsilon, v} \mid g_{\epsilon, w}\right\rangle=\langle\mathrm{C}(P)(0) v \mid w\rangle
$$

To complete the proof, we observe that

$$
\left\langle P f_{\epsilon, \nu, v} \mid f_{\epsilon,-\bar{\nu}, w}\right\rangle_{J}=\left\langle e^{-\nu} \tilde{P}_{\circ} e^{\nu} g_{\epsilon, \nu} \mid g_{\epsilon, w}\right\rangle .
$$

Moreover, in view of Lemma 12.13, the operator $e^{-\nu} \widetilde{P}_{\circ} e^{\nu}$ belongs to $\mathcal{D}_{\mathcal{O}}$, and has constant part equal to $e^{-\nu} \mathrm{C}(\tilde{P}) \circ e^{\nu}$. Hence $\mathrm{C}\left(e^{-\nu} \tilde{P} \circ e^{\nu}\right)(0)=\mathrm{C}(\tilde{P})(\nu)$. Now apply $(120)$, with $e^{-\nu} \widetilde{P} \circ e^{\nu}$ in place of $P$. 


\section{A limit behavior of the spherical Fourier trans- form}

Throughout this section $P \in \mathcal{P}_{\sigma}^{\min }$ will be fixed, and we will discuss the asymptotic behavior of the $\tau$-spherical Fourier transform $\mathcal{F}=\mathcal{F}_{P}$ in the following sense. Let $Q \in$ $\mathcal{P}_{\sigma}^{\min }, \varphi \in C_{c}^{\infty}\left(\mathfrak{a}_{\mathrm{q}}^{+}(Q)\right)$, and suppose that (115) holds. For $v \in V_{\tau}^{K \cap H_{\mathrm{M}}}, \nu \in i \mathfrak{a}_{\mathrm{q}}^{*}$ and $\epsilon>0$ we define $f_{\epsilon, \nu, v} \in C_{c}^{\infty}\left(A_{\mathrm{q}}^{+}(Q)\right) \otimes V_{\tau}^{K \cap H_{\mathrm{M}}}$ as in the previous section, by (116). We shall investigate the asymptotic behavior of $\mathcal{F} T_{Q}^{\dagger} f_{\epsilon, \nu, v}$ as $\epsilon \searrow 0$.

Our first result is the following. For $v \in V_{\tau}^{K \cap H_{\mathrm{M}}}$, let $\psi_{v} \in{ }^{\circ} \mathcal{C}_{1}(\tau) \simeq C^{\infty}\left(M / H_{\mathrm{M}}: \tau_{\mathrm{M}}\right)$ be defined by $\psi_{v}\left(e H_{\mathrm{M}}\right)=v$. Then the map $v \mapsto \psi_{v}$ inverts the map (58), hence is an isometry. Extend $\varphi$ by zero to a compactly supported function on $\mathfrak{a}_{\mathrm{q}}$, and let $\hat{\varphi}$ denote its Euclidean Fourier transform, defined as in (22). Define $\varphi_{\epsilon, \nu, v}: i \mathfrak{a}_{\mathrm{q}}^{*} \rightarrow{ }^{\circ} \mathcal{C}(\tau)$ by

$$
\varphi_{\epsilon, \nu, v}(\lambda)=\sum_{s \in W} \epsilon^{-d / 2} \hat{\varphi}\left(\frac{s \lambda-\nu}{\epsilon}\right) C_{Q \mid P}^{\circ}(s: \lambda)^{*} \psi_{v} .
$$

Let $p_{0}$ be the polynomial defined by $(62)$ with $R=0$, and let

$$
{ }^{\prime} p_{*}(\lambda):=\prod_{s \in W} p_{0}(s \lambda), \quad p_{*}(\lambda):=\overline{p_{*}(-\bar{\lambda})} .
$$

Proposition 13.1 There exists a $r>0$ such that for every $p \in S\left(\mathfrak{a}_{\mathrm{q}}\right)$ which is divisible by $p_{*}$ we have

$$
\left\|p\left(\mathcal{F} T_{Q}^{\dagger} f_{\epsilon, \nu, v}-\varphi_{\epsilon, \nu, v}\right)\right\|_{L^{2}\left(i \mathfrak{a}_{\mathrm{q}}^{*}\right) \otimes}{ }^{\circ} \mathcal{C}(\tau)=\mathrm{O}\left(e^{-r / \epsilon}\right) \quad(\epsilon \searrow 0)
$$

for all $v \in V_{\tau}^{K \cap H_{\mathrm{M}}}$ and $\nu \in i \mathfrak{a}_{\mathrm{q}}^{*}$.

Proof. Let $\psi \in{ }^{\circ} \mathcal{C}(\tau)$. Then for $\lambda \in i \mathfrak{a}_{\mathrm{q}}^{*}$ we have:

$$
\begin{aligned}
& \left\langle\left[\mathcal{F} T_{Q}^{\dagger} f_{\epsilon, \nu, v}\right](\lambda) \mid \psi\right\rangle \\
& \quad=\left\langle T_{Q}^{\dagger} f_{\epsilon, \nu, v} \mid E^{\circ}(\psi: \lambda)\right\rangle \\
& \quad=\int_{\mathfrak{a}_{q}^{+}(Q)} \epsilon^{d / 2} e^{\nu(X)} \varphi(\epsilon X)\left\langle v \mid J(\exp X)^{1 / 2} E^{\circ}(\psi: \lambda)(\exp X)\right\rangle d X .
\end{aligned}
$$

Put $C_{Q \mid P, 1}^{\circ}(s: \lambda):=\operatorname{pr}_{1} \circ C_{Q \mid P}^{\circ}(s: \lambda)$ and let

$$
E_{Q, 1}^{\circ}(\psi: \lambda)(m a)=\sum_{s \in W} a^{s \lambda}\left[C_{Q \mid P, 1}^{\circ}(s: \lambda) \psi\right](m) \quad\left(m \in M, a \in A_{\mathrm{q}}\right)
$$

be the $(Q, 1)$-principal part of the normalized Eisenstein integral (cf. [13], eqn. (56)). It depends analytically on $\lambda \in i \mathfrak{a}_{\mathrm{q}}^{*}$. Moreover, define the remainder term $R(\psi, \lambda): \mathfrak{a}_{\mathrm{q}}^{+}(Q) \rightarrow$ $V_{\tau}$ by

$$
J(\exp X)^{1 / 2} E^{\circ}(\psi: \lambda)(\exp X)=E_{Q, 1}^{\circ}(\psi: \lambda)(\exp X)+R(\psi, \lambda)(X) .
$$


Let $I_{1}(v, \nu, \epsilon, \psi, \lambda)$ and $I_{2}(v, \nu, \epsilon, \psi, \lambda)$ be the integrals obtained from the integral (122) if one replaces $J(\exp X)^{1 / 2} E^{\circ}(\psi: \lambda)(\exp X)$ by $E_{Q, 1}^{\circ}(\psi: \lambda)(\exp X)$ and $R(\psi, \lambda)(X)$ respectively. Then

$$
\left\langle\left[\mathcal{F} T_{Q}^{\dagger} f_{\epsilon, \nu, \nu}\right](\lambda) \mid \psi\right\rangle=I_{1}(v, \nu, \epsilon, \psi, \lambda)+I_{2}(v, \nu, \epsilon, \psi, \lambda) .
$$

We claim that $I_{1}(v, \nu, \epsilon, \psi, \lambda)=\left\langle\varphi_{\epsilon, \nu, v}(\lambda) \mid \psi\right\rangle$ and then it remains to estimate the integral $p(\lambda) I_{2}(v, \nu, \epsilon, \psi, \lambda)$, for all $\psi \in{ }^{\circ} \mathcal{C}(\tau)$.

To prove the claim we observe that

$$
\begin{aligned}
I_{1}(v, \nu, \epsilon, \psi, \lambda) & =\epsilon^{d / 2} \int_{\mathfrak{a}_{\mathrm{q}}} \sum_{s \in W} \varphi(\epsilon X) e^{(\nu-s \lambda)(X)}\left\langle v \mid C_{Q \mid P, 1}^{\circ}(s: \lambda) \psi(e)\right\rangle d X \\
& =\epsilon^{-d / 2} \sum_{s \in W} \hat{\varphi}\left(\frac{s \lambda-\nu}{\epsilon}\right)\left\langle v \mid C_{Q \mid P, 1}^{\circ}(s: \lambda) \psi(e)\right\rangle .
\end{aligned}
$$

Because the map $v \mapsto \psi_{v}$ is an isometry, we have:

$$
\left\langle v \mid C_{Q \mid P, 1}^{\circ}(s: \lambda) \psi(e)\right\rangle=\left\langle\psi_{v} \mid C_{Q \mid P, 1}^{\circ}(s: \lambda) \psi\right\rangle \text {. }
$$

Since $\psi_{v} \in{ }^{\circ} \mathcal{C}_{1}(\tau)=\operatorname{pr}_{1}{ }^{\circ} \mathcal{C}(\tau)$, the right-hand side in the above equation is equal to $\left\langle\psi_{v} \mid C_{Q \mid P}^{\circ}(s: \lambda) \psi\right\rangle=\left\langle C_{Q \mid P}^{\circ}(s: \lambda)^{*} \psi_{v} \mid \psi\right\rangle$ and the validity of the claim now follows.

The proof is now completed by the estimate of $p_{*}(\lambda) I_{2}(v, \nu, \epsilon, \psi, \lambda)$ given in the lemma below.

Lemma 13.2 There exists a $r>0$ and for every $\nu \in i \mathfrak{a}_{\mathrm{q}}^{*}, N>0$ a constant $C>0$ such that for all $v \in V_{\tau}^{K \cap H_{\mathrm{M}}}, \psi \in{ }^{\circ} \mathcal{C}(\tau), \lambda \in i \mathfrak{a}_{\mathrm{q}}^{*}$ and all $0<\epsilon \leq 1$ we have

$$
\left\|p_{*}(\lambda) I_{2}(v, \nu, \epsilon, \psi, \lambda)\right\| \leq C(1+|\lambda|)^{-N} e^{-r / \epsilon}\|\psi\|\|v\| .
$$

Proof. Since ${ }^{\circ} \mathcal{C}(\tau)$ is finite dimensional, it suffices to prove the estimate for a fixed $\psi$. From Theorem 7.5 and (59) with $w=1$, and in view of definition (60) and the expansion (61), we infer that the remainder term is given by the following absolutely convergent series $\left(\lambda \in i \mathfrak{a}_{\mathrm{q}}^{*}\right)$ :

$$
{ }^{\prime} p_{*}(\lambda) R(\psi, \lambda)(X)=\sum_{\substack{s \in W \\ \gamma \in \mathbb{N} \in(Q) \backslash\{0\}}} R_{s, \gamma}(\psi, \lambda)(X),
$$

where

$$
R_{s, \gamma}(\psi, \lambda)(X)={ }^{\prime} p_{*}(\lambda) e^{(s \lambda-\gamma)(X)}{ }_{\gamma}^{\sim}(s \lambda)\left[C_{Q \mid P, 1}^{\circ}(s: \lambda) \psi\right](e) .
$$

By Proposition 5.3 and Theorem 7.6 there exist constants $C^{\prime}>0$ and $\kappa>0$ such that

$$
\left\|{ }^{\prime} p_{*}(\lambda)^{\sim}{ }_{\gamma}(s \lambda)\left[C_{Q \mid P, 1}^{\circ}(s: \lambda) \psi\right](e)\right\| \leq C^{\prime}(1+|\lambda|)^{\operatorname{deg} p_{*}}(1+|\gamma|)^{\kappa},
$$

for all $\lambda \in i \mathfrak{a}_{\mathrm{q}}^{*}, \gamma \in \mathbb{N} \Sigma(Q)$.

Let

$$
r^{\prime}=\min \{\alpha(X) \mid \alpha \in \Sigma(Q), X \in \operatorname{supp} \varphi\}
$$


and let $0<r<r^{\prime}$. Then for every $\gamma \in \mathbb{N} \Sigma(Q)$ we have $\gamma \geq r^{\prime} m(\gamma)$ on $\operatorname{supp} \varphi$. Hence if $X \in \epsilon^{-1} \operatorname{supp} \varphi$, then

$$
\left\|R_{s, \gamma}(\psi, \lambda)(X)\right\| \leq C^{\prime}(1+|\lambda|)^{\operatorname{deg} p_{*}}(1+|\gamma|)^{\kappa} e^{-m(\gamma) r^{\prime} / \epsilon},
$$

for all $\lambda \in i \mathfrak{a}_{\mathrm{q}}^{*}, \gamma \in \mathbb{N} \Sigma(Q)$. Since $|\{\gamma \in \mathbb{N} \Sigma(Q) \mid m(\gamma)=k\}|$ grows at most linearly in $k$, the series

$$
G(z)=\sum_{\gamma \in \mathbb{N} \Sigma(Q) \backslash\{0\}}(1+|\gamma|)^{\kappa} z^{m(\gamma)}
$$

converges absolutely in the disc $|z|<1$, and defines an analytic function $G$ there. It follows from this that the series (125) converges absolutely and uniformly in $X \in \epsilon^{-1} \operatorname{supp} \varphi$, for every $\lambda \in i \mathfrak{a}_{\mathrm{q}}^{*}$, and $0<\epsilon \leq 1$. Interchanging integration and summation and performing the substitution $X \mapsto \epsilon^{-1} X$, we now obtain $\left(\lambda \in i \mathfrak{a}_{\mathrm{q}}^{*}\right)$ :

$$
p_{*}(\lambda) I_{2}(v, \nu, \epsilon, \psi, \lambda)=\sum_{\substack{s \in W \\ \gamma \in \mathbb{N} \Sigma(Q) \backslash\{0\}}} I_{s, \gamma}(v, \nu, \epsilon, \psi, \lambda),
$$

where

$$
I_{s, \gamma}(v, \nu, \epsilon, \psi, \lambda)=\epsilon^{-d / 2} \hat{\varphi}\left(\frac{s \lambda-\nu+\gamma}{\epsilon}\right)\left\langle v \mid{ }^{\prime} p_{*}(\lambda){ }_{\gamma}(s \lambda) C_{Q \mid P, 1}^{\circ}(s: \lambda) \psi(e)\right\rangle .
$$

Fix $\nu \in i \mathfrak{a}_{\mathrm{q}}^{*}$. By the Paley-Wiener estimate for the Euclidean Fourier-Laplace transform of a compactly supported smooth function there exists for every $N \in \mathbb{N}$ a constant $D>0$ such that

$$
\left|\hat{\varphi}\left(\frac{s \lambda-\nu+\gamma}{\epsilon}\right)\right| \leq D\left(1+\epsilon^{-1}|s \lambda-\nu+\gamma|\right)^{-N} e^{-m(\gamma) r^{\prime} / \epsilon},
$$

for all $0<\epsilon \leq 1, \lambda \in i \mathfrak{a}_{\mathrm{q}}^{*}, \gamma \in \mathbb{N} \Sigma(Q)$. Since $\gamma$ is real, whereas $s \lambda-\nu$ is purely imaginary, we have $|s \lambda-\nu+\gamma| \geq|s \lambda-\nu|$, hence there exists a constant $D^{\prime}$, depending on $N$ and $\nu$, such that

$$
\left|\hat{\varphi}\left(\frac{s \lambda-\nu+\gamma}{\epsilon}\right)\right| \leq D^{\prime}(1+|\lambda|)^{-N} e^{-m(\gamma) r^{\prime} / \epsilon}
$$

Applying this estimate and (126) to (128), we find that for every $N \in \mathbb{N}$ there exists a constant $D^{\prime \prime}>0$, such that for all $0<\epsilon \leq 1, \gamma \in \mathbb{N} \Sigma(Q) \backslash\{0\}$, and $v \in V_{\tau}^{K \cap H_{\mathrm{M}}}$ we have

$$
\left|I_{s, \gamma}(v, \nu, \epsilon, \psi, \lambda)\right| \leq D^{\prime \prime}(1+|\lambda|)^{-N}(1+|\gamma|)^{\kappa} \epsilon^{-d / 2} e^{-m(\gamma) r^{\prime} / \epsilon}\|v\| \quad\left(\lambda \in i \mathfrak{a}_{\mathrm{q}}^{*}\right) .
$$

We deduce from this that there exists a constant $D^{\prime \prime \prime}>0$ such that:

$$
\left|p_{*}(\lambda) I_{2}(v, \nu, \epsilon, \psi, \lambda)\right| \leq D^{\prime \prime \prime} \epsilon^{-d / 2}(1+|\lambda|)^{-N}\|v\| G\left(e^{-r^{\prime} / \epsilon}\right),
$$

for all $0<\epsilon \leq 1, \lambda \in i \mathfrak{a}_{\mathrm{q}}^{*}, v \in V_{\tau}^{K \cap H_{\mathrm{M}}}$. We now use that the holomorphic function $G$ vanishes at $z=0$, and therefore satisfies an estimate of the form $G(z)=\mathrm{O}(z)$ on $|z| \leq e^{-r^{\prime}}$. Since $r<r^{\prime}$ we infer that $\epsilon^{-d / 2} G\left(e^{-r^{\prime} / \epsilon}\right)=\mathrm{O}\left(e^{-r / \epsilon}\right)$, and the lemma follows. 
The following corollary to Proposition 13.1 will be crucial in the next section.

Corollary 13.3 Let $D \in \mathbb{D}(G / H)$ be a differential operator such that the polynomial $\lambda \mapsto p_{*}(\lambda) \overline{p_{*}(-\bar{\lambda})}$ divides $\mu(D: \tau)$ in $S\left(\mathfrak{a}_{\mathrm{q}}\right) \otimes \operatorname{End}\left({ }^{\circ} \mathcal{C}(\tau)\right)$. Then for all $v, w \in V_{\tau}^{K \cap H_{\mathrm{M}}}$ and $\nu \in i \mathfrak{a}_{\mathrm{q}}^{*}$ we have

$$
\lim _{\epsilon \backslash 0}\left\langle\mathcal{F} D T_{Q}^{\dagger} f_{\epsilon, \nu, v} \mid \mathcal{F} T_{Q}^{\dagger} f_{\epsilon, \nu, w}\right\rangle=\langle\underline{\mu}(D: \tau: \nu) v \mid w\rangle
$$

Proof. Write $I(\epsilon)$ for the inner product behind the limit sign in (130), and let $P \in$ $S\left(\mathfrak{a}_{\mathrm{q}}\right) \otimes \operatorname{End}\left({ }^{\circ} \mathcal{C}(\tau)\right)$ denote the quotient of $\mu(D: \tau: \lambda)$ by $p_{*}(\lambda) \overline{p_{*}(-\bar{\lambda})}$. Then for $\nu \in i \mathfrak{a}_{\mathrm{q}}^{*}$ we have

$$
I(\epsilon)=\left\langle P p_{\star} \mathcal{F} T_{Q}^{\dagger} f_{\epsilon, \nu, v} \mid p_{*} \mathcal{F} T_{Q}^{\dagger} f_{\epsilon, \nu, w}\right\rangle .
$$

From definition (121), the estimate (129) with $\gamma=0$, and the unitarity of the normalized $C$-functions (Proposition 5.3) it follows that for every polynomial $q \in S\left(\mathfrak{a}_{\mathrm{q}}\right)$ we have:

$$
\left\|q \varphi_{\epsilon, \nu, v}\right\|_{L^{2}\left(i \mathfrak{a}_{\mathrm{q}}^{*}\right) \otimes V_{\tau}^{K \cap H_{\mathrm{M}}}}=\mathrm{O}\left(\epsilon^{-d / 2}\right) \quad(\epsilon \searrow 0) .
$$

Combining this with Proposition 13.1 we see that

$$
I(\epsilon)=\left\langle P p_{*} \varphi_{\epsilon, \nu, v} \mid p_{*} \varphi_{\epsilon, \nu, w}\right\rangle+\mathrm{O}(\epsilon)=J(\epsilon)+\mathrm{O}(\epsilon),
$$

where $J(\epsilon)=\left\langle\mu(D: \tau) \varphi_{\epsilon, \nu, v} \mid \varphi_{\epsilon, \nu, w}\right\rangle$. Thus it suffices to study the limit behavior of $J(\epsilon)$. Substituting $(121)$ we see that $J(\epsilon)=\sum_{s, t \in W} J_{s, t}(\epsilon)$, with

$$
\begin{aligned}
J_{s, t}(\epsilon) & =\epsilon^{-d} \int_{i \mathfrak{a}_{\mathrm{q}}^{*}} \hat{\varphi}\left(\frac{s \lambda-\nu}{\epsilon}\right) \overline{\hat{\varphi}\left(\frac{t \lambda-\nu}{\epsilon}\right)} A_{s, t}(\lambda) d \lambda, \\
A_{s, t}(\lambda) & =\left\langle\mu(D: \tau: \lambda) C_{Q \mid P}^{\circ}(s: \lambda)^{*} \psi_{v} \mid C_{Q \mid P}^{\circ}(t: \lambda)^{*} \psi_{w}\right\rangle .
\end{aligned}
$$

Here we recall that $d \lambda=|W|^{-1} d \mu_{\mathrm{reg}}(\lambda)$ as in Section 3. Substituting $\xi=\epsilon^{-1}(s \lambda-\nu)$ we obtain

$$
J_{s, t}(\epsilon)=|W|^{-1} \int_{i \mathfrak{a}_{\mathrm{q}}^{*}} \hat{\varphi}(\xi) \overline{\hat{\varphi}\left(t s^{-1} \xi+\epsilon^{-1}\left(t s^{-1} \nu-\nu\right)\right)} A_{s, t}\left(\epsilon s^{-1} \xi+s^{-1} \nu\right) d \mu_{\mathrm{reg}}(\xi) .
$$

If $t s^{-1} \nu-\nu \neq 0$, then by dominated convergence we see that $\lim _{\epsilon} \searrow_{0} J_{s, t}(\epsilon)=0$ (use that $\hat{\varphi}$ is Schwartz, and that $A_{s, t}(\lambda)$ is polynomially bounded in $\lambda$, by unitarity of the normalized $C$-functions). On the other hand, if $t s^{-1} \nu=\nu$, then by dominated convergence we see that

$$
\begin{aligned}
\lim _{\epsilon \backslash 0} J_{s, t}(\epsilon) & =|W|^{-1} \int_{i \mathfrak{a}_{\mathrm{q}}^{*}} \hat{\varphi}(\xi) \overline{\hat{\varphi}\left(t s^{-1} \xi\right)} d \mu_{\mathrm{reg}}(\xi) A_{s, t}\left(s^{-1} \nu\right) \\
& =|W|^{-1} \int_{\mathfrak{a}_{\mathrm{q}}^{*}} \varphi(X) \overline{\varphi\left(t s^{-1} X\right)} d X A_{s, t}\left(s^{-1} \nu\right) \\
& =|W|^{-1} \delta_{s, t} A_{s, t}\left(s^{-1} \nu\right) .
\end{aligned}
$$


Therefore

$$
\begin{aligned}
\lim _{\epsilon \searrow 0} I(\epsilon) & =\lim _{\epsilon \searrow 0} J(\epsilon) \\
& =|W|^{-1} \sum_{s \in W} A_{s, s}\left(s^{-1} \nu\right) \\
& =|W|^{-1} \sum_{s \in W}\left\langle C_{Q \mid P}^{\circ}\left(s: s^{-1} \nu\right) \mu\left(D: \tau: s^{-1} \nu\right) C_{Q \mid P}^{\circ}\left(s: s^{-1} \nu\right)^{*} \psi_{v} \mid \psi_{w}\right\rangle \cdot(13
\end{aligned}
$$

Using (38) and Proposition 5.3 we now see that

$$
\lim _{\epsilon \backslash 0} I(\epsilon)=\left\langle\mu(D: \tau: \nu) \psi_{v} \mid \psi_{w}\right\rangle=\left\langle\psi_{\underline{\mu}(D: \tau: \nu) v} \mid \psi_{w}\right\rangle=\langle\underline{\mu}(D: \tau: \nu) v \mid w\rangle .
$$

Here we have used the definition of $\underline{\mu}$ in Section 7 and the fact that the map $v \mapsto \psi_{v}$ is an isometry.

\section{Fourier inversion modulo a differential operator}

In this section we shall use the results of the previous sections to prove the following theorem. Recall the definition of $\pi \in \Pi_{\Sigma}\left(\mathfrak{a}_{\mathrm{q}}\right)$ from $(64)$, and that of $\mathbb{D}_{\pi}=\mathbb{D}_{\tau, \pi} \subset \mathbb{D}(G / H)$ from Definition 10.3 with $p=\pi$. Then $\mathbb{D}_{\pi} \neq \emptyset$, by Corollary 10.4 .

Theorem 14.1 Let $D \in \mathbb{D}_{\pi}$. Then

$$
D \mathcal{J} \mathcal{F} f=D f
$$

for all $f \in C_{c}^{\infty}(G / H: \tau)$.

Proof. Fix $D \in \mathbb{D}_{\pi}$, and let $f \in C_{c}^{\infty}(G / H: \tau)$. Then the functions on both sides of (133) are smooth. Hence it suffices to prove (133) on $K A_{q}^{+}(Q) H$ for any $Q \in \mathcal{P}_{\sigma}^{\min }$ (use (5)). For this it suffices to show that $\langle g \mid D \mathcal{J} \mathcal{F} f\rangle=\langle g \mid D f\rangle$ for all $g \in C_{c}^{\infty}(G / H: \tau)$ with supp $g \subset K A_{\mathrm{q}}^{+}(Q) H$ (cf. Lemma 11.3). The latter equation is equivalent to

$$
\langle D \mathcal{J} \mathcal{F} g \mid f\rangle=\langle D g \mid f\rangle,
$$

by Corollary $9.4(\mathrm{~b})$ and since $D=D^{*}$ (see Definition 10.3 with $p=\pi$ ).

Thus it suffices to prove $D \mathcal{J} \mathcal{F} g=D g$ for every $g \in C_{c}^{\infty}(G / H: \tau)$ with $\operatorname{supp} g \subset$ $K A_{\mathrm{q}}^{+}(Q) H$. In other words, we have reduced the proof of (133) to the case that $f \in$ $C_{c}^{\infty}\left(K A_{\mathrm{q}}^{+}(Q) H: \tau\right)=\operatorname{image}\left(T_{Q}^{\dagger}\right)$ (see (49) and Lemma 7.1), with arbitrary $Q$.

Since $D \mathcal{J F}$ and $D$ decrease supports (cf. Theorem 11.1), the maps $D \mathcal{J} \mathcal{F} \circ T_{Q}^{\dagger}$ and $D \circ T_{Q}^{\dagger} \operatorname{map} C_{c}^{\infty}\left(A_{q}^{+}(Q), V_{\tau}^{K \cap H_{\mathrm{M}}}\right)$ into $C_{c}^{\infty}\left(K A_{\mathrm{q}}^{+}(Q) H: \tau\right)$. Hence by Lemma 7.1 it suffices to show that the endomorphism

$$
P:=T_{Q}^{\downarrow} \circ D \mathcal{J} \mathcal{F} \circ T_{Q}^{\dagger}
$$


of $C_{c}^{\infty}\left(A_{q}^{+}(Q), V_{\tau}^{K \cap H_{\mathrm{M}}}\right)$ equals $T_{Q}^{\downarrow} \circ D \circ T_{Q}^{\dagger}$. The latter operator equals $\Pi_{\tau}(D)=\Pi_{Q, \tau}(D)$. Thus it suffices to show that

$$
P=\Pi_{\tau}(D) .
$$

This identity will be established in the rest of this section.

Lemma 14.2 The operator $P=T_{Q}^{\downarrow} \circ D \mathcal{J F} \circ T_{Q}^{\dagger}$ is a differential operator with smooth End $\left(V_{\tau}^{K \cap H_{\mathrm{M}}}\right)$-valued coefficients, satisfying the commutation relations

$$
\left[P, \Pi_{\tau}(\partial)\right]=0 \quad(\partial \in \mathbb{D}(G / H))
$$

Proof. It follows from Theorem 11.1 that the continuous linear operator $P$ from the space $C_{c}^{\infty}\left(A_{q}^{+}(Q), V_{\tau}^{K \cap H_{\mathrm{M}}}\right)$ to the space $C^{\infty}\left(A_{q}^{+}(Q), V_{\tau}^{K \cap H_{\mathrm{M}}}\right)$ decreases supports. Hence $P$ is a differential operator with smooth $\operatorname{End}\left(V_{\tau}^{K \cap H_{\mathrm{M}}}\right)$-valued coefficients (cf. [30], remark on p. 236). Let $\partial \in \mathbb{D}(G / H)$ be arbitrary. Then it follows from Corollary 9.4 (a) that $\partial$ commutes with the operator $D \mathcal{J} \mathcal{F}$. From this and $(51)$ we see that $P$ commutes with $T_{Q}^{\downarrow} \circ \partial \circ T_{Q}^{\dagger}$, which equals $\Pi_{\tau}(\partial)$.

It follows from the above lemma and Theorem 12.3 that $P \in \mathcal{D}_{\mathcal{O}}$, and that the constant part $\mathrm{C}(P) \in \operatorname{End}\left(V_{\tau}^{K \cap H_{\mathrm{M}}}\right) \otimes S\left(\mathfrak{a}_{\mathrm{q}}\right)$ is well defined and determines $P$ uniquely. Thus, in order to obtain the identity (135) we need only to verify that $\mathrm{C}(P)=\mathrm{C}\left(\Pi_{\tau}(D)\right)$, or equivalently, that

$$
\mathrm{C}(\tilde{P})=\mathrm{C}\left(\widetilde{\Pi_{\tau}(D)}\right) .
$$

Observe that

$$
\mathrm{C}\left(\widetilde{\Pi_{\tau}(D)}\right)=\underline{\mu}(D: \tau)
$$

by Lemmas 12.2 and 12.14. Hence (136) is a consequence of the proposition below, which completes the proof of Theorem 14.1.

Proposition 14.3 C $(\widetilde{P})=\underline{\mu}(D: \tau)$.

Proof. Let $p_{*}$ be as in Proposition 13.1. Then by Lemma 10.7 there exists a differential operator $D_{*} \in \mathbb{D}(G / H)$ such that the polynomial $\lambda \mapsto p_{*}(\lambda) \overline{p_{*}(-\bar{\lambda})}$ divides $\mu\left(D_{*}: \tau\right)$ in $S\left(\mathfrak{a}_{q}\right) \otimes \operatorname{End}\left({ }^{\circ} \mathcal{C}(\tau)\right)$, and such that $\operatorname{det} \mu\left(D_{*}: \tau\right)$ is non-trivial. In particular, $\mu\left(D_{*}: \tau\right)$ is not a zero divisor in $S\left(\mathfrak{a}_{\mathrm{q}}\right) \otimes \operatorname{End}\left(V_{\tau}^{K \cap H_{\mathrm{M}}}\right)$. Now consider the operator $P^{\prime}=T_{Q}^{\downarrow} D_{*} D \mathcal{J} \mathcal{F} T_{Q}^{\dagger}$. Using $(51)$ we infer that $P^{\prime}=\Pi_{\tau}\left(D_{*}\right) P$, and by Lemmas 12.1 and 12.2 we see that $P^{\prime} \in \mathcal{D}_{\mathcal{O}}$ and

$$
\mathrm{C}\left(\widetilde{P}^{\prime}\right)=\underline{\mu}\left(D_{*}: \tau\right) \mathrm{C}(\widetilde{P}) .
$$

On the other hand, in view of Lemma 12.15 we have, for $v, w \in V_{\tau}^{K \cap H_{\mathrm{M}}}, \nu \in i \mathfrak{a}_{\mathrm{q}}^{*}$,

$$
\left\langle\mathrm{C}\left(\tilde{P}^{\prime}\right)(\nu) v \mid w\right\rangle=\lim _{\epsilon \searrow 0}\left\langle P^{\prime} f_{\epsilon, \nu, v} \mid f_{\epsilon, \nu, w}\right\rangle_{J}
$$




$$
\begin{aligned}
& =\lim _{\epsilon \backslash 0}\left\langle T_{Q}^{\downarrow} D_{*} D \mathcal{J} \mathcal{F} T_{Q}^{\dagger} f_{\epsilon, \nu, v} \mid f_{\epsilon, \nu, w}\right\rangle_{J} \\
& =\lim _{\epsilon \backslash 0}\left\langle\mathcal{J F} D_{*} D T_{Q}^{\dagger} f_{\epsilon, \nu, v} \mid T_{Q}^{\dagger} f_{\epsilon, \nu, w}\right\rangle \\
& =\lim _{\epsilon \backslash 0}\left\langle\mathcal{F} D_{*} D T_{Q}^{\dagger} f_{\epsilon, \nu, v} \mid \mathcal{F} T_{Q}^{\dagger} f_{\epsilon, \nu, w}\right\rangle \\
& =\left\langle\underline{\mu}\left(D_{*} D: \tau: \nu\right) v \mid w\right\rangle,
\end{aligned}
$$

the last three equalities being in consequence of (53) and Corollary 9.4 (a), (82), and Corollary 13.3, respectively. Hence

$$
\mathrm{C}\left(\tilde{P}^{\prime}\right)=\underline{\mu}\left(D_{*}: \tau\right) \underline{\mu}(D: \tau) .
$$

The desired conclusion now follows from (138) and (139), since $\underline{\mu}\left(D_{*}: \tau\right)$ is not a zero divisor.

Remark 14.4 Consider again the case where $G / H$ is a Riemannian symmetric space of the non-compact type. Then the Eisenstein integrals (see also [31], Ch. III, $\S 2$ ) are entire functions on $a_{q c}^{*}$. The normalized Eisenstein integrals are obtained from these by division with Harish-Chandra's c-function (use loc.cit., p. 245, eqn. (51)), which by the formula of Gindikin-Karpelevic has no zeros in $\mathfrak{a}_{\mathrm{q}}^{*}(\bar{P}, 0)$, and hence the polynomial $\pi$ of $(64)$ is 1 . By Remark 10.9 we have $1 \in D_{\pi}$, hence Theorem 14.1 says that $\mathcal{J} \mathcal{F}=I$ on $C_{c}^{\infty}(G / H: \tau)$. In this case our formula is essentially identical with the inversion formula for Helgason's $\delta$-spherical Fourier transform, see loc.cit., Ch. III, Prop. 5.10 and Thm. 5.16.

\section{Injectivity of the Fourier transforms}

From the inversion theorem (Theorem 14.1) of the previous section we will deduce injectivity of the Fourier transforms $\mathcal{F}$ and $f \mapsto \hat{f}$ on compactly supported smooth functions.

Theorem 15.1 The Fourier transform $\mathcal{F}$ maps $C_{c}^{\infty}(G / H: \tau)$ injectively into the space $\mathcal{S}\left(i \mathfrak{a}_{\mathrm{q}}^{*}\right) \otimes{ }^{\circ} \mathcal{C}(\tau)$.

The proof of this theorem will be based on a result of [12], which we will now recall.

Fix $Q \in \mathcal{P}_{\sigma}^{\min }$ and put $\mathfrak{n}=\mathfrak{n}_{Q}$. In view of the decomposition $\mathfrak{g}=\mathfrak{n}+\mathfrak{m}+\mathfrak{a}_{\mathrm{q}}+\mathfrak{h}$, we may define a map ' $\eta_{Q}: U(\mathfrak{g}) \rightarrow U\left(\mathfrak{a}_{\mathrm{q}}\right)$ by ' $\eta_{Q}(u) \equiv u \operatorname{modulo}(\mathfrak{n}+\mathfrak{m}) U(\mathfrak{g})+U(\mathfrak{g}) \mathfrak{h}$. By restriction to $U(\mathfrak{g})^{H}$ we obtain a homomorphism $\mathbb{D}(G / H) \rightarrow S\left(\mathfrak{a}_{\mathrm{q}}\right)$, also denoted by ' $\eta_{Q}$. We define the homomorphism $\eta: \mathbb{D}(G / H) \rightarrow S\left(\mathfrak{a}_{\mathrm{q}}\right)$ by $\eta(D)(\lambda)={ }^{\prime} \eta_{Q}(D)\left(\lambda+\rho_{Q}\right)(D \in$ $\left.\mathbb{D}(G / H), \lambda \in \mathfrak{a}_{\mathrm{qc}}^{*}\right)$; then $\eta$ is independent of the choice of $Q$, cf. [12], Lemma 1.

Recall that if $S$ is a closed subset of $\mathfrak{a}_{\mathrm{q}}$, then we define the subset $X_{S}$ of $G / H$ by (66). 
Proposition 15.2 Let $D \in \mathbb{D}(G / H)$ be a non-zero differential operator with deg $\eta(D)=$ order $D$, and let $S$ be a $W_{K \cap H}$-invariant closed convex subset of $\mathfrak{a}_{\mathrm{q}}$. Then for every $f \in$ $C_{c}^{\infty}(G / H)$ we have:

$$
\operatorname{supp} D f \subset X_{S} \Rightarrow \operatorname{supp} f \subset X_{S} .
$$

In particular, $D$ is injective on $C_{c}^{\infty}(G / H)$.

Proof. This is Theorem 2 of [12]. The final statement is equivalent to (140) with $S=\emptyset$.

Lemma 15.3 Let $p \in \Pi\left(\mathfrak{a}_{\mathrm{q}}\right)$, and define the subset $\mathbb{D}_{p}^{\prime}=\mathbb{D}_{\tau, p}^{\prime}$ of $\mathbb{D}(G / H)$ by

$$
\mathbb{D}_{p}^{\prime}=\left\{D \in \mathbb{D}_{p} \mid \operatorname{deg} \eta(D)=\text { order } D\right\}
$$

Then $\mathbb{D}_{p}^{\prime} \neq \emptyset$.

Proof. From [12], Lemma 3, we see that for $D \in \mathbb{D}(G / H)$ we have $\operatorname{deg} \eta(D)=\operatorname{deg} \eta\left(D^{*}\right)$. Fix $w \in \mathcal{W}$. Then from the first lines of the proof of Corollary 10.4 we see that we must show that in Theorem 10.2 the operator $D=D_{w}$ can be found with $\operatorname{deg} \eta(D)=\operatorname{order}(D)$. This will be true if the operator $D$ in Lemma 10.7 can be found such that $\operatorname{deg} \eta(D)=$ order $D$. We will go over the proof of that lemma, and verify that the constructed operator $D$ satisfies the additional requirement. One easily verifies that one may still reduce to the case that $p \in \Pi\left(\mathfrak{a}_{\mathrm{q}}\right)$ is of the form $p=X+c$, with $X \in \mathfrak{a}_{\mathrm{q} c} \backslash\{0\}, c \in \mathbb{C}$. Define $q \in S(\mathfrak{j})^{W(\mathfrak{j})}$ and $Z \in \mathfrak{Z}$ as in the proof. Then we must show that $D=r(Z)$ satisfies the additional requirement.

Fix $Q \in \mathcal{P}_{\sigma}^{\min }$, put $\mathfrak{n}=\mathfrak{n}_{Q}$, and define ' $\eta_{Q}$ as above. Then $\operatorname{deg} \eta(\partial)=\operatorname{deg}$ ' $\eta_{Q}(\partial)$ for all $\partial \in \mathbb{D}(G / H)$. Now ' $\eta_{Q}(D)={ }^{\prime} \eta_{Q}(Z)$, and since $\operatorname{deg} ' \eta_{Q}(Z) \leq \operatorname{order} D \leq \operatorname{order} Z=\operatorname{deg} q$, it is sufficient to show that $\operatorname{deg}{ }^{\prime} \eta_{Q}(Z)=\operatorname{deg} q$. We recall that the Cartan subalgebra $j$ decomposes as in (19), and that, accordingly, $\mathfrak{a}_{\mathrm{qc}}^{*}$ and $\mathfrak{j}_{0 \mathbf{c}}^{*}$ are viewed as subspaces of $\mathfrak{j}_{\mathbf{c}}^{*}$. Let $\Sigma^{+}(\mathfrak{j})$ be a choice of positive roots for $\mathfrak{j}_{\mathbf{c}}$ in $\mathfrak{g}_{\mathbf{c}}$ which is compatible with $\Sigma(Q)$. Let $\mathfrak{g}_{\mathbf{c}}^{+}$be the associated sum of positive root spaces in $\mathfrak{g}_{\mathbf{c}}$, and let $\rho_{\mathbf{j}} \in \mathfrak{j}_{\mathbf{c}}^{*}$ be the associated rho. Define ' $q \in S(\mathfrak{j})$ by ${ }^{\prime} q(\Lambda)=q\left(\Lambda-\rho_{\mathfrak{j}}\right)$ for $\Lambda \in \mathfrak{j}_{\mathbf{c}}^{*}$. Then it suffices to show that $\operatorname{deg}{ }^{\prime} q=\operatorname{deg}{ }^{\prime} \eta_{Q}(Z)$. Now $Z \equiv{ }^{\prime} q$ modulo $\mathfrak{g}_{\mathbf{c}}^{+} U(\mathfrak{g})$. Since

$$
\mathfrak{g}_{\mathbf{c}}^{+}=\mathfrak{n}_{\mathbf{c}} \oplus\left[\mathfrak{g}_{\mathbf{c}}^{+} \cap \mathfrak{m}_{\mathbf{c}}\right]
$$

it follows from this that ' $\eta_{Q}(Z)$ equals the restriction of ' $q$ to $\mathfrak{a}_{\mathrm{qc}}^{*}$. Now ' $q$ is a product of first degree polynomials of the form $L: \Lambda \mapsto\left(w \Lambda-w \rho_{j}+\Lambda_{\xi}\right)(X+Y)+c$. Since $w^{-1}(X+Y) \notin \mathfrak{j}_{0}$ by Lemma 10.6 , the polynomial $\lambda \mapsto w \lambda(X+Y)$ is not constant on $\mathfrak{a}_{\mathrm{q} c}^{*}$. The restriction of every factor $L$ to $\mathfrak{a}_{\mathrm{q} c}^{*}$ is therefore of degree 1 . Hence the restriction of ' $q$ to $\mathfrak{a}_{\mathrm{q}}^{*} \mathrm{c}$ has the same degree as ' $q$, and the proof is complete.

Proof of Theorem 15.1. Let $f \in C_{c}^{\infty}(G / H: \tau)$, and suppose that $\mathcal{F} f=0$. Fix $D \in \mathbb{D}_{\pi}^{\prime}$ (cf. the lemma above). Then from Theorem 14.1 it follows that $D \mathcal{J F} f=D f$, hence $D f=0$. Applying Proposition 15.2 we infer that $f=0$. 
Using Proposition 15.2 once more we obtain the following version of the inversion formula.

Corollary 15.4 Let $D \in \mathbb{D}(G / H)$ be any differential operator such that $D \mathcal{J F}$ maps $C_{c}^{\infty}(G / H: \tau)$ to itself. Then $D \mathcal{J} \mathcal{F}=D$ on $C_{c}^{\infty}(G / H: \tau)$.

Proof. Fix $D_{1} \in \mathbb{D}_{\pi}^{\prime}$, and let $f \in C_{c}^{\infty}(G / H: \tau)$. Then it follows from Theorem 14.1 that $D_{1}(D \mathcal{J} \mathcal{F} f-D f)=D\left(D_{1} \mathcal{J} \mathcal{F} f-D_{1} f\right)=0$. But $D \mathcal{J} \mathcal{F} f-D f$ is compactly supported, hence using Proposition 15.2 we infer that it is zero.

From Theorem 15.1 we obtain the following companion result for the Fourier transform $f \mapsto \hat{f}$.

Theorem 15.5 Let $f \in C_{c}^{\infty}(G / H)$. If $\hat{f}(\xi, \lambda)=0$ for all $[\xi] \in \widehat{M}_{H}$ and $\lambda \in i \mathfrak{a}_{\mathrm{q}}^{*}$, then $f=0$.

Proof. By equivariance and continuity of the Fourier transform (cf. Lemma 4.3) it suffices to prove this for a $K$-finite $f$. Thus assume that $\vartheta \subset \widehat{K}$ is finite, and that $f \in C_{c}^{\infty}(G / H)_{\vartheta}$. Let $\tau=\tau_{\vartheta}$ be as in Remark 5.1 and recall the definition of $\varsigma$ in (43). We recall that $\varsigma$ maps $C_{c}^{\infty}(G / H)_{\vartheta}$ bijectively onto $C_{c}^{\infty}(G / H: \tau)$. Put $F=\varsigma(f)$. Then by Proposition 6.4 we have $\mathcal{F} F=0$, and hence $F=0$ by Theorem 15.1. This implies that $f=0$ as well.

We end this section with a result that relates $\eta(D)$ to the $Q$-radial component $\Pi_{Q, \tau}(D)$ defined in (54), with $Q$ a given parabolic subgroup in $\mathcal{P}_{\sigma}^{\min }$. Recall that $\Pi_{Q, \tau}(D)$ is a differential operator on $A_{\mathrm{q}}^{+}(Q)$, with coefficients in $C^{\infty}\left(A_{\mathrm{q}}^{+}(Q)\right) \otimes \operatorname{End}\left(V_{\tau}^{K \cap H_{\mathrm{M}}}\right)$.

Lemma 15.6 Let $D \in \mathbb{D}(G / H)$. Then order $D=\operatorname{deg} \eta(D)$ if and only if order $D=$ order $\Pi_{Q, \tau}(D)$. Moreover, if one of these equivalent conditions is fulfilled, then the principal symbol of $\Pi_{Q, \tau}(D)$ equals $I_{V_{\tau}^{K \cap H_{\mathrm{M}}}} \otimes\left[{ }^{\prime} \eta_{\bar{Q}}(D)\right]_{d}$; here the subscript $d$ indicates that the homogeneous part of degree $d:=\operatorname{order} D$ has been taken.

Proof. Assume that order $(D)=d$, with $d \geq 1$ (otherwise all assertions are trivial). Fix $\tilde{D} \in U(g)^{H}$ of order $d$ such that $r(\tilde{D})=D$. Put $\overline{\mathfrak{n}}=\overline{\mathfrak{n}}_{Q}$. Then we have the decomposition $\mathfrak{g}=\overline{\mathfrak{n}}+(\mathfrak{m} \cap \mathfrak{k})+\mathfrak{a}_{\mathfrak{q}}+\mathfrak{h}$, as a sum of vector spaces. Hence $\tilde{D}-{ }^{\prime} \eta_{\bar{Q}}(D)$ belongs to $\overline{\mathfrak{n}} U(\mathfrak{g})_{d-1}+(\mathfrak{m} \cap \mathfrak{k}) U(\mathfrak{g})_{d-1}+U(\mathfrak{g}) \mathfrak{h}$, where $U(\mathfrak{g})_{d-1}$ denotes the subspace of $U(\mathfrak{g})$ consisting of all elements of order at most $d-1$. It now follows from [4], Lemma 3.9, that the differential operator $\Pi_{Q, \tau}(D)-I \otimes{ }^{\prime} \eta_{\bar{Q}}(D)$ has order at most $d-1$. From this all assertions follow.

\section{The image of the spherical Fourier transform}

In this section we will determine the image of the Schwartz space under the Fourier transform $\mathcal{F}=\mathcal{F}_{P}$, and the kernel of the wave packet transform $\mathcal{J}=\mathcal{J}_{P}$ (as before we assume that $P \in \mathcal{P}_{\sigma}^{\mathrm{min}}$, and that $\tau$ is a finite dimensional unitary representation of $K$ ). 
If $s, t \in W$, then from [13], eqn. (71), we see that

$$
C_{P \mid P}^{\circ}(t: s \lambda) C_{P \mid P}^{\circ}(s: \lambda)=C_{P \mid P}^{\circ}(t s: \lambda) \quad\left(\lambda \in i \mathfrak{a}_{\mathrm{q}}^{*}\right) .
$$

This condition allows us to define a representation $\gamma$ of $W$ on the space of ${ }^{\circ} \mathcal{C}(\tau)$-valued functions on $i \mathfrak{a}_{\mathrm{q}}^{*}$, by

$$
[\gamma(s) \varphi](\lambda)=C_{P \mid P}^{\circ}\left(s^{-1}: \lambda\right)^{-1} \varphi\left(s^{-1} \lambda\right)=C_{P \mid P}^{\circ}\left(s: s^{-1} \lambda\right) \varphi\left(s^{-1} \lambda\right) .
$$

Notice that it follows from the unitarity of the normalized $C$-functions, see Proposition 5.3 , that $\gamma$ defines a unitary representation of $W$ on $L^{2}\left(i \mathfrak{a}_{\mathrm{q}}^{*}\right) \otimes{ }^{\circ} \mathcal{C}(\tau)$.

Lemma 16.1 Let $s \in W$. Then $\gamma(s)$ commutes with $\mu(D: \tau)$ for all $D \in \mathbb{D}(G / H)$, that is

$$
\gamma(s)(\mu(D: \tau) \circ \varphi)=\mu(D: \tau) \circ(\gamma(s) \varphi)
$$

for all $\varphi$ as above.

Proof. This follows from eqn. (38).

The importance of the representation $\gamma$ is apparent from the following result. Let $\left(\mathcal{S}\left(i \mathfrak{a}_{\mathrm{q}}^{*}\right) \otimes{ }^{\circ} \mathcal{C}(\tau)\right)^{W}$ denote the space of $\gamma(W)$-invariants in $\mathcal{S}\left(i \mathfrak{a}_{\mathrm{q}}^{*}\right) \otimes{ }^{\circ} \mathcal{C}(\tau)$. Clearly this is a closed subspace of $\mathcal{S}\left(i \mathfrak{a}_{\mathrm{q}}^{*}\right) \otimes{ }^{\circ} \mathcal{C}(\tau)$, hence it is Fréchet.

Lemma 16.2 The Fourier transform $\mathcal{F}$ maps $\mathcal{C}(G / H: \tau)$ into $\left(\mathcal{S}\left(i \mathfrak{a}_{\mathrm{q}}^{*}\right) \otimes{ }^{\circ} \mathcal{C}(\tau)\right)^{W}$.

Proof. This is an immediate consequence of Lemma 6.3 with $P_{1}=P_{2}=P$.

Remark 16.3 Although we did not assert it in the above lemma, it is true that $\mathcal{S}\left(i \mathfrak{a}_{\mathrm{q}}^{*}\right) \otimes$ ${ }^{\circ} \mathcal{C}(\tau)$ is an invariant subspace for $\gamma$, and that $\gamma(s)$ acts continuously on it for all $s \in W$. This follows from polynomial estimates for the derivatives of the $C$-functions that can be obtained using the material of [7]. However, we shall not need this here.

In this section we will show that we actually have the equality

$$
\operatorname{im}(\mathcal{F})=\left(\mathcal{S}\left(i \mathfrak{a}_{\mathrm{q}}^{*}\right) \otimes{ }^{\circ} \mathcal{C}(\tau)\right)^{W}
$$

In the proof we will need the following result, which is the analogue of [27], Thm. 26.1.

Theorem 16.4 The wave packet transform $\mathcal{J}$ maps $\mathcal{S}\left(i \mathfrak{a}_{\mathrm{q}}^{*}\right) \otimes{ }^{\circ} \mathcal{C}(\tau)$ continuously into the Schwartz space $\mathcal{C}(G / H: \tau)$.

Proof. See [9], Thm. 1. 
Combining this theorem with the continuity of $\mathcal{F}$ on the Schwartz space (cf. Lemma 6.1) and density of $C_{c}^{\infty}(G / H: \tau)$ in $\mathcal{C}(G / H: \tau)$, we see that Theorem 14.1 holds for Schwartz functions:

Corollary 16.5 Let $D \in \mathbb{D}_{\pi}$. Then $D \mathcal{J F}=D$ on $\mathcal{C}(G / H: \tau)$.

We now come to a result that will be the key to the other results of this section. Since $\gamma$ defines a unitary representation of $W$ in the Hilbert space $L^{2}\left(i \mathfrak{a}_{\mathrm{q}}^{*}\right) \otimes{ }^{\circ} \mathcal{C}(\tau)$, the map

$$
\mathrm{P}_{\mathrm{W}}: f \mapsto|W|^{-1} \sum_{s \in W} \gamma(s) f
$$

defines an equivariant orthogonal projection operator onto the space of $W$-invariants, and we have an orthogonal direct sum decomposition of $L^{2}\left(i \mathfrak{a}_{\mathrm{q}}^{*}\right) \otimes{ }^{\circ} \mathcal{C}(\tau)$ into the following invariant closed subspaces:

$$
L^{2}\left(i \mathfrak{a}_{\mathrm{q}}^{*}\right) \otimes{ }^{\circ} \mathcal{C}(\tau)=\operatorname{ker} \mathrm{P}_{\mathrm{W}} \oplus\left(L^{2}\left(i \mathfrak{a}_{\mathrm{q}}^{*}\right) \otimes{ }^{\circ} \mathcal{C}(\tau)\right)^{W}
$$

Theorem 16.6 On $\mathcal{S}\left(i \mathfrak{a}_{\mathrm{q}}^{*}\right) \otimes{ }^{\circ} \mathcal{C}(\tau)$ we have $\mathcal{F} \mathcal{J}=\mathrm{P}_{\mathrm{W}}$.

We will prove Theorem 16.6 in a number of steps. First we collect some properties of the composition $\mathcal{F} \mathcal{J}$.

Lemma 16.7 The composition $\mathcal{T}=\mathcal{F} \mathcal{J}$ is a continuous linear endomorphism of $\mathcal{S}\left(i \mathfrak{a}_{\mathrm{q}}^{*}\right) \otimes$ ${ }^{\circ} \mathcal{C}(\tau)$. Moreover, it satisfies the following conditions (a) - (c).

(a) $\mu(D: \tau) \circ \mathcal{T}=\mathcal{T} \circ \mu(D: \tau)$ for all $D \in \mathbb{D}(G / H)$;

(b) $\gamma(s) \circ \mathcal{T}=\mathcal{T}$ for all $s \in W$;

(c) $\left\langle\mathcal{T} \varphi_{1} \mid \varphi_{2}\right\rangle=\left\langle\varphi_{1} \mid \mathcal{T} \varphi_{2}\right\rangle$ for all $\varphi_{1}, \varphi_{2} \in \mathcal{S}\left(i \mathfrak{a}_{\mathrm{q}}^{*}\right) \otimes{ }^{\circ} \mathcal{C}(\tau)$.

Proof. The continuity of $\mathcal{T}$ follows from Theorem 16.4 and Lemma 6.1. Condition (a) follows from Lemmas 9.1 and 6.2. Condition (b) follows from Lemma 16.2. Finally, (c) follows from Lemma 9.3 and Theorem 16.4.

Proposition 16.8 There exists an open dense $W$-invariant set $\mathfrak{a}_{\mathrm{q}}^{* \circ} \subset \mathfrak{a}_{\mathrm{q}}^{*}$ with the following property. Let $\mathcal{T}$ be any continuous linear endomorphism of $\mathcal{S}\left(i \mathfrak{a}_{\mathrm{q}}^{*}\right) \otimes{ }^{\circ} \mathcal{C}(\tau)$ such that conditions (a) - (c) of Lemma 16.7 are fulfilled. Then there exists a unique smooth function $\beta: i \mathfrak{a}_{\mathrm{q}}^{* 0} \rightarrow \operatorname{End}\left({ }^{\circ} \mathcal{C}(\tau)\right)$ such that

$$
\mathcal{T} \varphi(\lambda)=\beta(\lambda) \circ \mathrm{P}_{\mathrm{W}} \varphi(\lambda)
$$

for all $\varphi \in \mathcal{S}\left(i \mathfrak{a}_{\mathrm{q}}^{*}\right) \otimes{ }^{\circ} \mathcal{C}(\tau), \lambda \in i \mathfrak{a}_{\mathrm{q}}^{* 0}$. 
Proof. We will prove this result in a number of steps. For every $\lambda \in \mathfrak{a}_{\mathrm{qc}}^{*}$ we define the distribution $u_{\lambda} \in \mathcal{D}^{\prime}\left(i \mathfrak{a}_{\mathrm{q}}^{*}\right) \otimes \operatorname{End}\left({ }^{\circ} \mathcal{C}(\tau)\right)$ by

$$
u_{\lambda}(\varphi)=\mathcal{T} \varphi(\lambda) \quad\left(\varphi \in C_{c}^{\infty}\left(i \mathfrak{a}_{\mathrm{q}}^{*}\right) \otimes{ }^{\circ} \mathcal{C}(\tau)\right)
$$

Then the commutation relations (a) become:

$$
u_{\lambda} \circ \mu(D: \tau)=\mu(D: \tau: \lambda) \circ u_{\lambda} \quad(D \in \mathbb{D}(G / H)) .
$$

To cast these relations in a more tractable form, we recall the decomposition (39) of ${ }^{\circ} \mathcal{C}(\tau)$ in joint eigenspaces for $\mu(D: \tau: \lambda), D \in \mathbb{D}(G / H)$, where $L \subset i \mathfrak{b}_{\mathrm{k}}^{*}$ is finite. Here $\mathfrak{b}=\mathfrak{b}_{\mathrm{k}} \oplus \mathfrak{a}_{\mathrm{q}}$ is a Cartan subspace of $\mathfrak{q}$ containing $\mathfrak{a}_{\mathrm{q}}$. Via this decomposition we identify $\mathfrak{a}_{\mathrm{q} c}^{*}$ and $\mathfrak{b}_{\mathrm{k} c}^{*}$ with subspaces of $\mathfrak{b}_{\mathfrak{c}}^{*}$.

Let $W(\mathfrak{b})$ be the Weyl group of the restricted root system $\Sigma(\mathfrak{b})$ of $\mathfrak{b}_{\mathbf{c}}$ in $\mathfrak{g}_{\mathbf{c}}$. We claim that there exists an open subset $\Omega_{1} \subset i \mathfrak{a}_{q}^{*}$, whose complement is a finite union of hyperplanes, such that for all $\lambda_{1} \in \Omega_{1}, \lambda_{2} \in \mathfrak{a}_{\mathrm{qc}}^{*}$ we have

$$
L+\lambda_{1} \cap W(\mathfrak{b})\left[L+\lambda_{2}\right] \neq \emptyset \Rightarrow \lambda_{1} \in W \lambda_{2}
$$

In order to prove this claim we fix a basis $B$ for $\mathfrak{b}_{\mathrm{k} \mathfrak{c}}^{*}$. For $\Lambda_{1}, \Lambda_{2} \in L, w \in W(\mathfrak{b})$ and $\beta \in B$ with $w \beta \notin \mathfrak{b}_{\mathrm{k} \mathfrak{c}}^{*}$ the set

$$
\left\{\lambda \in i \mathfrak{a}_{\mathbf{q}}^{*} \mid\langle\lambda, w \beta\rangle=\left\langle w \Lambda_{2}-\Lambda_{1}, w \beta\right\rangle\right\}
$$

is either empty or a hyperplane in $i \mathfrak{a}_{\mathrm{q}}^{*}$. Let $\Omega_{1} \subset i \mathfrak{a}_{\mathrm{q}}^{*}$ be the complement of the union of all these sets. Then if $\lambda_{1} \in \Omega_{1}$ and $\Lambda_{1}+\lambda_{1}=w\left(\Lambda_{2}+\lambda_{2}\right)$ for some $\Lambda_{1}, \Lambda_{2} \in L, w \in W(\mathfrak{b})$ and $\lambda_{2} \in \mathfrak{a}_{\mathrm{qc}}^{*}$ we have $\left\langle\lambda_{1}, w \beta\right\rangle=\left\langle w\left(\Lambda_{2}+\lambda_{2}\right)-\Lambda_{1}, w \beta\right\rangle=\left\langle w \Lambda_{2}-\Lambda_{1}, w \beta\right\rangle$, and hence by the definition of $\Omega_{1}, w \beta \in \mathfrak{b}_{\mathrm{k}}^{*}$, for all $\beta \in B$. It follows that $w$ normalizes $\mathfrak{b}_{\mathrm{k}}$, and hence also $\mathfrak{a}_{\mathrm{q}}$. Moreover it then follows that $\lambda_{1}=w \lambda_{2}$ and $\Lambda_{1}=w \Lambda_{2}$. This proves (147).

The next steps in the proof of Proposition 8.3 are given in the following lemmas. Recall that $\gamma: \mathbb{D}(G / H) \rightarrow S(\mathfrak{b})$ is the Harish-Chandra homomorphism, and that $\gamma_{\Lambda}(D: \lambda):=$ $\gamma(D: \Lambda+\lambda)$ for $\Lambda \in \mathfrak{b}_{\mathrm{k} \boldsymbol{c}}^{*}$ and $\lambda \in \mathfrak{a}_{\mathrm{q} c}^{*}$.

Lemma 16.9 If $\lambda \in \Omega_{1}$, then $\operatorname{supp} u_{\lambda} \subset W \lambda$.

Proof. Fix $\Lambda_{1}, \Lambda_{2} \in L, \psi_{j} \in{ }^{\circ} \mathcal{C}(\tau)\left[\Lambda_{j}\right](j=1,2)$, and define $v \in \mathcal{D}^{\prime}\left(i \mathfrak{a}_{\mathrm{q}}^{*}\right)$ by $v(f)=$ $\left\langle u_{\lambda}\left(f \otimes \psi_{1}\right) \mid \psi_{2}\right\rangle$ for $f \in C_{c}^{\infty}\left(i \mathfrak{a}_{\mathrm{q}}^{*}\right)$. Then it suffices to show that $\operatorname{supp} v \subset W \lambda$.

The relations (146) imply that

$$
\left[\gamma_{\Lambda_{1}}(D)-\gamma_{\Lambda_{2}}(D)(\lambda)\right] v=0
$$

for all $D \in \mathbb{D}(G / H)$. Let $\nu \in \operatorname{supp} v$. Then it follows from (148) that $\gamma\left(D: \Lambda_{1}+\nu\right)=$ $\gamma\left(D: \Lambda_{2}+\lambda\right)$, for all $D \in \mathbb{D}(G / H)$. This implies that $\Lambda_{2}+\lambda$ is $W(\mathfrak{b})$-conjugate to $\Lambda_{1}+\nu$, hence that $\nu \in W \lambda$ by the property (147) of $\Omega_{1}$. Hence $\operatorname{supp} v \subset W \lambda$. 
Our next objective is to show that, for generic $\lambda$, the distribution $u_{\lambda}$ has order zero. The following lemma will be our basic tool.

Lemma 16.10 Let $u$ be a distribution on $\mathbb{R}^{n}$ with support consisting of a single point $x_{0} \in \mathbb{R}^{n}$. Moreover, let $\varphi_{j} \in C^{\infty}\left(\mathbb{R}^{n}\right)(1 \leq j \leq n)$ and suppose that the $d \varphi_{j}\left(x_{0}\right), 1 \leq j \leq$ $n$, are linearly independent. Finally assume that

$$
\varphi_{j} u=\varphi_{j}\left(x_{0}\right) u \quad(1 \leq j \leq n) .
$$

Then $u$ is a multiple of the Dirac measure $\delta_{x_{0}}$ at $x_{0}$.

Proof. The proof is straightforward, and involves an application of the multi-variable Leibniz rule.

Lemma 16.11 Let $\Lambda \in \mathfrak{b}_{\mathrm{k} c}^{*}$. Then for $\lambda$ in the complement in $\mathfrak{a}_{\mathrm{qc}}^{*}$ of a finite union of hyperplanes, the map from $\mathbb{D}(G / H)$ to $\mathfrak{a}_{\mathrm{q}} \mathbf{c}$, given by $D \mapsto d\left[\gamma_{\Lambda}(D)\right](\lambda)$, is surjective.

Proof. For $\alpha \in \Sigma(\mathfrak{b})$, let $H_{\alpha} \in \mathfrak{b}_{\mathfrak{c}}$ be the -1 eigenvector for the reflection $s_{\alpha}$ in $\alpha$, normalized by $\alpha\left(H_{\alpha}\right)=2$. If $S \subset \mathfrak{b}_{\mathfrak{c}}^{*}$, we write $W(\mathfrak{b}, S)$ for the centralizer of $S$ in $W(\mathfrak{b})$, and $\Sigma(\mathfrak{b}, S)$ for the set of roots $\alpha \in \Sigma(\mathfrak{b})$ whose root hyperplane ker $H_{\alpha}$ in $\mathfrak{b}_{\mathfrak{c}}^{*}$ contains $S$. (Here and in the following we use the canonical identification $\mathfrak{b}_{\mathbf{c}} \simeq \mathfrak{b}_{\mathbf{c}}^{* *}$.) It is well known that $W(\mathfrak{b}, S)$ is the subgroup of $W(\mathfrak{b})$ generated by the reflections in roots of $\Sigma(\mathfrak{b}, S)$. Since obviously $\Sigma\left(\mathfrak{b}, \Lambda+\mathfrak{a}_{\mathrm{q} c}^{*}\right)=\Sigma\left(\mathfrak{b}, \mathbb{C} \Lambda+\mathfrak{a}_{\mathrm{q} c}^{*}\right)$, it follows that the group $W\left(\mathfrak{b}, \Lambda+\mathfrak{a}_{\mathrm{q}}^{*}\right)$ equals $W\left(\mathfrak{b}, \mathbb{C} \Lambda+\mathfrak{a}_{\mathrm{q} c}^{*}\right)$, hence in particular centralizes $\mathfrak{a}_{\mathrm{q}}^{*}$.

Let $\Omega$ be the subset of $\lambda \in \mathfrak{a}_{\mathrm{qc}}^{*}$ for which $\Lambda+\lambda$ is not contained in any of the root hyperplanes $\operatorname{ker}\left(H_{\beta}\right), \beta \in \Sigma(\mathfrak{b}) \backslash \Sigma\left(\mathfrak{b}, \Lambda+\mathfrak{a}_{\mathrm{q} \mathfrak{c}}^{*}\right)$. Then $\Omega$ is the complement of finitely many hyperplanes in $\mathfrak{a}_{\mathrm{qc}}^{*}$. Moreover, by definition we have, for $\lambda \in \Omega$, that $\Sigma(\mathfrak{b}, \Lambda+\lambda)=$ $\Sigma\left(\mathfrak{b}, \Lambda+\mathfrak{a}_{\mathrm{q}}^{*}\right)$ and hence $W(\mathfrak{b}, \Lambda+\lambda)$ centralizes $\mathfrak{a}_{\mathrm{q}}^{*}$.

Fix $\lambda \in \Omega$ and let $X \in \mathfrak{a}_{\mathrm{q}}$ be given. Choose a polynomial function $p: \mathfrak{b}_{\mathbf{c}}^{*} \rightarrow \mathbb{C}$ such that

$$
d p(w(\Lambda+\lambda))=w X
$$

for all $w \in W(\mathfrak{b})$. This is possible because the stabilizer of $\Lambda+\lambda$ in $W(\mathfrak{b})$ stabilizes $X$ by what was said above. The polynomial

$$
q(\nu):=\frac{1}{|W(\mathfrak{b})|} \sum_{w \in W(\mathfrak{b})} p(w \nu)
$$

has derivative $d q(\nu)=|W(\mathfrak{b})|^{-1} \sum_{w \in W(\mathfrak{b})} w^{-1} d p(w \nu)$, hence

$$
d q(\Lambda+\lambda)=X
$$

On the other hand, $q$ is $W(\mathfrak{b})$-invariant, hence equal to $\gamma(D)$ for some $D \in \mathbb{D}(G / H)$. Let $\pi_{\mathfrak{a}_{\mathrm{q}}}$ denote the projection $\mathfrak{b}_{\mathbf{c}} \rightarrow \mathfrak{a}_{\mathrm{q}} \mathbf{c}$ along $\mathfrak{b}_{\mathbf{k}}$; then it follows that

$$
d\left[\gamma_{\Lambda}(D)\right](\lambda)=\pi_{\mathfrak{a}_{\mathrm{q}}}[d q(\Lambda+\lambda)]=X .
$$


We shall now use Lemmas 16.9 and 16.11 to prove that for generic $\lambda$ the distribution $u_{\lambda}$ defined by (145) is a sum of Dirac measures. More precisely, let $\Omega_{1}$ be as in Lemma 16.9 , and let $\Omega_{2}$ be a complement in $i \mathfrak{a}_{\mathrm{q}}^{*}$ of a finite union of hyperplanes such that the assertion of Lemma 16.11 holds for every $\Lambda \in L, \lambda \in \Omega_{2}$. Let $\Omega_{0}$ denote the intersection of $\Omega_{1} \cap \Omega_{2}$ with the set of regular points in $i \mathfrak{a}_{\mathrm{q}}^{*}$, and finally let $i \mathfrak{a}_{\mathrm{q}}^{* 0}$ be the intersection of the sets $w \Omega_{0},(w \in W)$. Then the complement of $i \mathfrak{a}_{\mathrm{q}}^{* 0}$ in $i \mathfrak{a}_{\mathrm{q}}^{*}$ is a finite union of hyperplanes.

Lemma 16.12 For every $\lambda \in i \mathfrak{a}_{\mathrm{q}}^{* 0}$ the distribution $u_{\lambda}$ is supported by $W \lambda$ and has order zero.

Proof. Fix $\lambda \in i \mathfrak{a}_{\mathrm{q}}^{* 0}$. Then $u_{\lambda}$ is supported in $W \lambda$, by Lemma 16.9. Let $\Lambda_{j} \in L, \psi_{j} \in$ ${ }^{\circ} \mathcal{C}(\tau)\left[\Lambda_{j}\right]$ for $j=1,2$, and define $v$ as in the proof of that lemma. Since $\lambda$ is regular, the orbit $W \lambda$ consists of $|W|$ distinct points and we may express $v$ uniquely as a sum of distributions $v_{s}(s \in W)$, with $\operatorname{supp} v_{s} \subset\{s \lambda\}$. From (148) it follows that each $v_{s}$ satisfies the relations

$$
\varphi_{D} v_{s}=0
$$

where $\varphi_{D}=\gamma_{\Lambda_{1}}(D)-\gamma_{\Lambda_{2}}(D)(\lambda)$. It follows from the definition of $i \mathfrak{a}_{\mathrm{q}}^{* 0}$ and Lemma 16.11 that the collection of differentials $d \varphi_{D}(s \lambda) \quad(D \in \mathbb{D}(G / H))$ spans $\mathfrak{a}_{\mathrm{q}}^{*}$, and therefore contains a subset consisting of $\operatorname{dim} \mathfrak{a}_{\mathrm{qc}}^{*}$ independent elements. Now apply Lemma 16.10 to conclude that each $v_{s}$ has order zero.

Completion of the proof of Proposition 16.8. It follows from Lemma 16.12 that there exist unique functions $E_{s}: i \mathfrak{a}_{\mathrm{q}}^{* 0} \rightarrow \operatorname{End}\left({ }^{\circ} \mathcal{C}(\tau)\right)$ such that for every $\lambda \in i \mathfrak{a}_{\mathrm{q}}^{* 0}$ we have

$$
u_{\lambda}=\sum_{s \in W} \delta_{s \lambda} \otimes E_{s}(\lambda)
$$

In particular, if $\psi \in C_{c}^{\infty}\left(i \mathfrak{a}_{\mathrm{q}}^{* 0}\right) \otimes{ }^{\circ} \mathcal{C}(\tau)$ is supported inside a given chamber, we have

$$
\mathcal{T} \psi(\lambda)=u_{\lambda}(\psi)=E_{1}(\lambda) \psi(\lambda)
$$

for $\lambda$ in this chamber, and hence by the property (b) of $\mathcal{T}$ (in Lemma 16.7) that

$$
\mathcal{T} \psi(\lambda)=\sum_{s \in W} \gamma(s)\left[E_{1} \psi\right](\lambda)
$$

for all $\lambda \in i \mathfrak{a}_{\mathrm{q}}^{* 0}$.

Let $\varphi \in \mathcal{S}\left(i \mathfrak{a}_{\mathrm{q}}^{*}\right) \otimes{ }^{\circ} \mathcal{C}(\tau)$ be given and fix $\lambda \in i \mathfrak{a}_{\mathrm{q}}^{* 0}$. Let $\psi \in C_{c}^{\infty}\left(i \mathfrak{a}_{\mathrm{q}}^{* 0}\right) \otimes{ }^{\circ} \mathcal{C}(\tau)$ be supported inside the chamber containing $\lambda$, then it follows from property (c) (in Lemma 16.7) and eqn. (149) that

$$
\langle\mathcal{T} \varphi \mid \psi\rangle=\langle\varphi \mid \mathcal{T} \psi\rangle=\left\langle\varphi \mid \sum_{s \in W} \gamma(s)\left[E_{1} \psi\right]\right\rangle=\left\langle E_{1}^{*} \sum_{s \in W} \gamma(s) \varphi \mid \psi\right\rangle
$$

Hence (144) follows with $\beta=|W| E_{1}^{*}$.

It is easily seen from (144) that $\beta$ is unique and smooth. 
Proof of Theorem 16.6. In view of Lemma 16.7 the operator $\mathcal{T}=\mathcal{F} \mathcal{J}$ satisfies the conclusion of Proposition 16.8. It remains to prove that we have $\beta=I$ for this $\mathcal{T}$; the resulting equation

$$
\mathcal{T} \varphi(\lambda)=\mathrm{P}_{\mathrm{W}} \varphi(\lambda)
$$

for $\lambda \in i \mathfrak{a}_{\mathrm{q}}^{* 0}$ extends to $i \mathfrak{a}_{\mathrm{q}}^{*}$ by continuity.

Let $f \in \mathcal{C}(G / H: \tau)$ and apply $(144)$ to $\varphi=\mathcal{F} f$. Then we have

$$
\mathcal{F} \mathcal{J} \mathcal{F} f(\lambda)=\beta(\lambda) \mathcal{F} f(\lambda)
$$

for $\lambda \in i \mathfrak{a}_{\mathrm{q}}^{* 0}$. Select $D \in \mathbb{D}_{\pi}$ (recall that this set is non-empty, by Corollary 10.4). Then $\operatorname{det} \mu(D: \tau)$ belongs to $\Pi\left(\mathfrak{a}_{\mathrm{q}}\right)$ hence does not vanish identically. Multiplying (150) with $\mu(D: \tau: \lambda)$ and applying Corollary 16.5 and Lemma 6.2 we infer that

$$
\mu(D: \tau: \lambda) \mathcal{F} f(\lambda)=\mu(D: \tau: \lambda) \beta(\lambda) \mathcal{F} f(\lambda)
$$

for $\lambda \in i \mathfrak{a}_{\mathrm{q}}^{* \circ}$. Using Lemma 16.13 below, and the fact that $\mu(D: \tau: \lambda)$ is invertible for $\lambda$ in a dense open subset of $i \mathfrak{a}_{\mathrm{q}}^{*}$, we now conclude that $\beta(\lambda)=I$ for $\lambda$ in a dense open subset of $i \mathfrak{a}_{\mathrm{q}}^{* 0}$. By continuity of $\beta$ this completes the proof.

Lemma 16.13 Let $\lambda \in i \mathfrak{a}_{\mathrm{q}}^{*}$, and suppose that $\langle\lambda, \alpha\rangle \neq 0$ for all $\alpha \in \Sigma$. Then $f \mapsto \mathcal{F} f(\lambda)$ maps $C_{c}^{\infty}(G / H: \tau)$ onto ${ }^{\circ} \mathcal{C}(\tau)$.

Proof. Let $\lambda$ satisfy the hypotheses, and suppose that $\psi \in{ }^{\circ} \mathcal{C}(\tau)$ is orthogonal to $\mathcal{F} f(\lambda)$ for every $f \in C_{c}^{\infty}(G / H: \tau)$. We will show that then $\psi=0$.

We have

$$
\left\langle f \mid E^{\circ}(P: \psi: \lambda)\right\rangle=\langle\mathcal{F} f(\lambda) \mid \psi\rangle=0,
$$

for all $f \in C_{c}^{\infty}(G / H: \tau)$. This implies that $E^{\circ}(P: \psi: \lambda)$ vanishes on $G / H$. Now apply the lemma below.

Lemma 16.14 Let $\lambda \in i \mathfrak{a}_{\mathrm{q}}^{*}$, and suppose that $\langle\lambda, \alpha\rangle \neq 0$ for all $\alpha \in \Sigma$. Then for every $\psi \in{ }^{\circ} \mathcal{C}(\tau) \backslash\{0\}$ the function $E^{\circ}(P: \psi: \lambda)$ does not vanish identically on $G / H$.

Proof. Let $\lambda$ satisfy the hypotheses, let $\psi \in{ }^{\circ} \mathcal{C}(\tau)$, and suppose that $E^{\circ}(P: \psi: \lambda)=0$. If $w \in \mathcal{W}$, then on $A_{\mathrm{q}}^{+}(P) w H$ the Eisenstein integral is asymptotically given by its principal part:

$$
a^{\rho_{P}} E^{\circ}(P: \psi: \lambda)(a w)=\sum_{s \in W} a^{s \lambda}\left[C_{P \mid P}^{\circ}(s: \lambda) \psi\right]_{w}(e)+o(1)
$$

as $a \rightarrow \infty$ along rays in $A_{\mathrm{q}}^{+}(P)$ (see Remark 5.5). Since $\lambda$ is regular, the exponents $s \lambda(s \in$ $W)$ are distinct, and by uniqueness of asymptotics we conclude that each coefficient $\left[C_{P \mid P}^{\circ}(s: \lambda) \psi\right]_{w}(e)$ must be zero. Recalling that $C_{P \mid P}^{\circ}(1: \lambda)=I$, we conclude that $\psi_{w}(e)=$ 0 for all $w \in \mathcal{W}$. Hence $\psi(e)=0$, and since $\psi$ is $\tau_{\mathrm{M}}$-spherical this implies that $\psi=0$. 
If $V$ is a topological linear space, then by a projection of $V$ we mean an idempotent continuous linear endomorphism $P$ of $V$. For such an endomorphism we have ker $P=$ $\operatorname{im}(I-P)$ and $\operatorname{im} P=\operatorname{ker}(I-P)$, and $V=\operatorname{ker} P \oplus \operatorname{im} P$ as a direct sum of closed linear subspaces.

Corollary 16.15 The map $\mathrm{P}_{\mathrm{W}}$ leaves the space $\mathcal{S}\left(i \mathfrak{a}_{\mathrm{q}}^{*}\right) \otimes{ }^{\circ} \mathcal{C}(\tau)$ invariant. Its restriction $\mathbf{P}_{\mathrm{W}}$ to this space equals $\mathcal{F} \mathcal{J}$ and is a projection. The image of $\mathbf{P}_{\mathrm{W}}$ equals $\left(\mathcal{S}\left(i \mathfrak{a}_{\mathrm{q}}^{*}\right) \otimes\right.$ $\left.{ }^{\circ} \mathcal{C}(\tau)\right)^{W}$. Finally, $\mathbf{P}_{\mathrm{W}}$ is symmetric with respect to the $L^{2}$-pre-Hilbert structure of $\mathcal{S}\left(i \mathfrak{a}_{\mathrm{q}}^{*}\right) \otimes$ ${ }^{\circ} \mathcal{C}(\tau)$

Proof. The first assertion and the equality $\mathbf{P}_{\mathrm{W}}=\mathcal{F} \mathcal{J}$ follow from Theorem 16.6. That $\mathbf{P}_{\mathrm{W}}$ is a projection of $\mathcal{S}\left(i \mathfrak{a}_{\mathrm{q}}^{*}\right) \otimes{ }^{\circ} \mathcal{C}(\tau)$ is now obvious; the characterization of its image is obvious as well. The symmetry of $\mathbf{P}_{\mathrm{W}}$ follows from the orthogonality of $\mathrm{P}_{\mathrm{W}}$.

In particular we have the following decomposition of the Schwartz space into an orthogonal direct sum of closed subspaces:

$$
\mathcal{S}\left(i \mathfrak{a}_{\mathrm{q}}^{*}\right) \otimes{ }^{\circ} \mathcal{C}(\tau)=\operatorname{ker} \mathbf{P}_{\mathrm{W}} \oplus\left(\mathcal{S}\left(i \mathfrak{a}_{\mathrm{q}}^{*}\right) \otimes{ }^{\circ} \mathcal{C}(\tau)\right)^{W}
$$

We are now able to determine the image of $\mathcal{F}$ and the kernel of $\mathcal{J}$.

Theorem 16.16 The image of $\mathcal{F}: \mathcal{C}(G / H: \tau) \rightarrow \mathcal{S}\left(i \mathfrak{a}_{\mathrm{q}}^{*}\right) \otimes{ }^{\circ} \mathcal{C}(\tau)$ and the kernel of $\mathcal{J}: \mathcal{S}\left(i \mathfrak{a}_{\mathrm{q}}^{*}\right) \otimes{ }^{\circ} \mathcal{C}(\tau) \rightarrow \mathcal{C}(G / H: \tau)$ are given by

$$
\begin{aligned}
\operatorname{im} \mathcal{F} & =\operatorname{im} \mathbf{P}_{\mathrm{W}}=\left(\mathcal{S}\left(i \mathfrak{a}_{\mathrm{q}}^{*}\right) \otimes{ }^{\circ} \mathcal{C}(\tau)\right)^{W}, \\
\operatorname{ker} \mathcal{J} & =\operatorname{ker} \mathbf{P}_{\mathrm{W}} .
\end{aligned}
$$

Proof. Lemma 16.2 asserts that $\operatorname{im} \mathcal{F} \subset\left(\mathcal{S}\left(i \mathfrak{a}_{\mathrm{q}}^{*}\right) \otimes{ }^{\circ} \mathcal{C}(\tau)\right)^{W}$. On the other hand, from Theorem 16.6 it follows that $\mathcal{F} \mathcal{J}=I$ on $\left(\mathcal{S}\left(i \mathfrak{a}_{\mathrm{q}}^{*}\right) \otimes{ }^{\circ} \mathcal{C}(\tau)\right)^{W}$, establishing the converse inclusion and hence (151).

By (82) the kernel of $\mathcal{J}$ is the orthocomplement of the image of $\mathcal{F}$, hence (152) follows.

\section{The kernel of the spherical Fourier transform}

In this section we will study the kernel of $\mathcal{F}$ and the image of $\mathcal{J}$ in $\mathcal{C}(G / H: \tau)$. The main result is Corollary 17.4. We start with the following observation.

Lemma 17.1 The operator $\mathbf{P}_{\mathrm{mc}}:=\mathcal{J} \mathcal{F}$ is a projection of $\mathcal{C}(G / H: \tau)$. Moreover, it is symmetric with respect to the $L^{2}$-pre-Hilbert structure of $\mathcal{C}(G / H: \tau)$.

Proof. The continuity of $\mathbf{P}_{\mathrm{mc}}$ follows from Lemma 6.1 and Theorem 16.4. Combining Theorem 16.6 and Lemma 16.2 we see that

$$
\mathcal{F} \mathcal{J} \mathcal{F}=\mathcal{F}
$$

The idempotence of $\mathbf{P}_{\mathrm{mc}}$ now follows immediately. Finally, the symmetry is a straightforward consequence of Lemma 9.3. 
Let

$$
\begin{array}{ll}
\mathcal{C}_{0}(G / H: \tau) & :=\operatorname{ker}\left(\mathbf{P}_{\mathrm{mc}}\right), \\
\mathcal{C}_{\mathrm{mc}}(G / H: \tau) & :=\operatorname{ker}\left(I-\mathbf{P}_{\mathrm{mc}}\right)=\operatorname{im}\left(\mathbf{P}_{\mathrm{mc}}\right) .
\end{array}
$$

Then as a direct consequence of the above lemma we obtain:

Corollary 17.2 The following is an orthogonal direct sum decomposition into closed subspaces:

$$
\mathcal{C}(G / H: \tau)=\mathcal{C}_{\mathrm{mc}}(G / H: \tau) \oplus \mathcal{C}_{0}(G / H: \tau)
$$

Proposition 17.3 The kernel of $\mathcal{F}: \mathcal{C}(G / H: \tau) \rightarrow \mathcal{S}\left(i \mathfrak{a}_{\mathrm{q}}^{*}\right) \otimes{ }^{\circ} \mathcal{C}(\tau)$ and the image of $\mathcal{J}: \mathcal{S}\left(i \mathfrak{a}_{\mathrm{q}}^{*}\right) \otimes{ }^{\circ} \mathcal{C}(\tau) \rightarrow C^{\infty}(G / H: \tau)$ are given by:

$$
\begin{aligned}
\operatorname{ker} \mathcal{F} & =\mathcal{C}_{0}(G / H: \tau), \\
\operatorname{im} \mathcal{J} & =\mathcal{C}_{\mathrm{mc}}(G / H: \tau) .
\end{aligned}
$$

Moreover, let $D \in \mathbb{D}_{\pi}$. Then

$$
\operatorname{ker} D \cap \mathcal{C}(G / H: \tau)=\mathcal{C}_{0}(G / H: \tau) .
$$

Proof. From (153) we see that $\operatorname{ker} \mathcal{F}=\operatorname{ker} \mathcal{F} \mathcal{J} \mathcal{F} \supset \operatorname{ker} \mathcal{J} \mathcal{F} \supset \operatorname{ker} \mathcal{F}$, and the first identity follows.

To prove the second identity we note that im $\mathcal{J} \supset \operatorname{im} \mathcal{J F}=\operatorname{im} \mathbf{P}_{\text {mc }}=\mathcal{C}_{\text {mc }}(G / H: \tau)$. On the other hand, by (82) the image of $\mathcal{J}$ is contained in the orthocomplement of the kernel of $\mathcal{F}$, which by the first identity is $\mathcal{C}_{\mathrm{mc}}(G / H: \tau)$.

For the last equality, notice that $D=D \mathbf{P}_{\mathrm{mc}}$, by Corollary 16.5 , whence $\operatorname{ker} D \supset$ $\mathcal{C}_{0}(G / H: \tau)$. For the converse inclusion suppose that $f \in \operatorname{ker} D$. Then $\mu(D: \tau) \mathcal{F} f=$ $\mathcal{F}(D f)=0$. The endomorphism $\mu(D: \tau: \lambda)$ is invertible for $\lambda$ in a dense open subset of $i \mathfrak{a}_{\mathrm{q}}^{*}$, by Definition $10.3(\mathrm{~b})$ with $p=\pi$. It now follows that $\mathcal{F} f$ vanishes on an open dense subset of $i \mathfrak{a}_{\mathrm{q}}^{*}$, hence everywhere by its continuity. Hence $\operatorname{ker} D \subset \operatorname{ker} \mathcal{F}=\mathcal{C}_{0}(G / H: \tau)$.

Corollary 17.4 The restriction $\mathcal{F}_{\mathrm{mc}}=\mathcal{F} \mid \mathcal{C}_{\mathrm{mc}}(G / H: \tau)$ is a continuous linear isomorphism from $\mathcal{C}_{\mathrm{mc}}(G / H: \tau)$ onto $\left(\mathcal{S}\left(i \mathfrak{a}_{\mathrm{q}}^{*}\right) \otimes{ }^{\circ} \mathcal{C}(\tau)\right)^{W}$. The inverse of $\mathcal{F}_{\mathrm{mc}}$ equals the restriction of $\mathcal{J}$ to $\left(\mathcal{S}\left(i \mathfrak{a}_{\mathrm{q}}^{*}\right) \otimes{ }^{\circ} \mathcal{C}(\tau)\right)^{W}$. Finally, the map $\mathcal{F}_{\mathrm{mc}}$ is an isometry for the given $L^{2}$-pre-Hilbert structures on the Schwartz spaces.

Proof. That $\mathcal{F}_{\mathrm{mc}}$ is a bijection onto $\left(\mathcal{S}\left(i \mathfrak{a}_{\mathrm{q}}^{*}\right) \otimes{ }^{\circ} \mathcal{C}(\tau)\right)^{W}$, whose inverse is the restriction of $\mathcal{J}$ to this space, is immediate from the definitons and results above. Since $\mathcal{F}$ and $\mathcal{J}$ are continuous for the Schwartz space topologies, it follows that $\mathcal{F}_{\text {mc }}$ is a topological isomorphism. Thus, it remains to prove that $\mathcal{F}_{\mathrm{mc}}$ is an isometry. For this we notice that for $f \in \mathcal{C}_{\text {mc }}(G / H: \tau)$ we have $\mathcal{J} \mathcal{F} f=f$, hence

$$
\langle f \mid f\rangle=\langle\mathcal{J} \mathcal{F} f \mid f\rangle=\langle\mathcal{F} f \mid \mathcal{F} f\rangle .
$$


Let $L^{2}(G / H: \tau)$ denote the space of $\tau$-spherical square integrable functions $G / H \rightarrow$ $V_{\tau}$, and let $L^{2}\left(i \mathfrak{a}_{\mathrm{q}}^{*}\right) \otimes{ }^{\circ} \mathcal{C}(\tau)$ denote the space of square integrable functions $i \mathfrak{a}_{\mathrm{q}}^{*} \rightarrow{ }^{\circ} \mathcal{C}(\tau)$. Then $L^{2}(G / H: \tau)$ and $L^{2}\left(i \mathfrak{a}_{\mathrm{q}}^{*}\right) \otimes{ }^{\circ} \mathcal{C}(\tau)$ are the completions of the pre-Hilbert spaces $\mathcal{C}(G / H: \tau)$ and $\mathcal{S}\left(i \mathfrak{a}_{\mathrm{q}}^{*}\right) \otimes{ }^{\circ} \mathcal{C}(\tau)$ respectively. Let $L_{0}^{2}(G / H: \tau)$ and $L_{\mathrm{mc}}^{2}(G / H: \tau)$ be the closures in $L^{2}(G / H: \tau)$ of $\mathcal{C}_{0}(G / H: \tau)$ and $\mathcal{C}_{\mathrm{mc}}(G / H: \tau)$ respectively.

Corollary 17.5 We have the following orthogonal decomposition of Hilbert spaces:

$$
L^{2}(G / H: \tau)=L_{0}^{2}(G / H: \tau) \oplus L_{\mathrm{mc}}^{2}(G / H: \tau) .
$$

Moreover, the Fourier transform $\mathcal{F}$ has a unique extension to a continuous linear map from $L^{2}(G / H: \tau)$ to $L^{2}\left(i \mathfrak{a}_{\mathrm{q}}^{*}\right) \otimes{ }^{\circ} \mathcal{C}(\tau)$, also denoted by $\mathcal{F}$. Its kernel equals $L_{0}^{2}(G / H: \tau)$, and it maps $L_{\mathrm{mc}}^{2}(G / H: \tau)$ isometrically onto $\left(L^{2}\left(i \mathfrak{a}_{\mathrm{q}}^{*}\right) \otimes{ }^{\circ} \mathcal{C}(\tau)\right)^{W}$.

The adjoint $\mathcal{F}^{*}: L^{2}\left(i \mathfrak{a}_{\mathrm{q}}^{*}\right) \otimes{ }^{\circ} \mathcal{C}(\tau) \rightarrow L^{2}(G / H: \tau)$ of the extension $\mathcal{F}$ is the unique continuous linear extension of $\mathcal{J}: \mathcal{S}\left(i \mathfrak{a}_{\mathrm{q}}^{*}\right) \otimes{ }^{\circ} \mathcal{C}(\tau) \rightarrow \mathcal{C}(G / H: \tau)$. Finally, $\mathcal{F}^{*} \mathcal{F}$ is the orthogonal projection $L^{2}(G / H: \tau) \rightarrow L_{\mathrm{mc}}^{2}(G / H: \tau)$.

Proof. Apart from the assertion about the adjoint, all assertions are immediate consequences of the above discussion. Let $\varphi \in \mathcal{S}\left(i \mathfrak{a}_{\mathrm{q}}^{*}\right) \otimes{ }^{\circ} \mathcal{C}(\tau)$. Then for all $f \in \mathcal{C}(G / H: \tau)$ we have $\left\langle\mathcal{F}^{*} \varphi \mid f\right\rangle=\langle\varphi \mid \mathcal{F} f\rangle=\langle\mathcal{J} \varphi \mid f\rangle$, by Lemma 9.3. By density of $\mathcal{C}(G / H: \tau)$ in $L^{2}(G / H: \tau)$ we now see that $\mathcal{F}^{*}=\mathcal{J}$ on $\mathcal{S}\left(i \mathfrak{a}_{\mathrm{q}}^{*}\right) \otimes{ }^{\circ} \mathcal{C}(\tau)$.

The space $\mathcal{C}_{0}(G / H: \tau)$ is spectrally small in the sense that it equals the kernel of any differential operator from $\mathbb{D}_{\pi}$. In a distribution sense a similar assertion is valid for $L_{0}^{2}(G / H: \tau)$.

Lemma 17.6 Let $D \in \mathbb{D}_{\pi}=\mathbb{D}_{\tau, \pi}$, and let ker $D$ denote the space of generalized functions $G / H \rightarrow V_{\tau}$ annihilated by $D$. Then

$$
L_{0}^{2}(G / H: \tau)=L^{2}(G / H: \tau) \cap \operatorname{ker} D .
$$

Proof. Let $f \in L^{2}(G / H: \tau)$. If $g \in \mathcal{C}(G / H: \tau)$, then $\langle D f \mid g\rangle=\langle f \mid D g\rangle=\langle f \mid D \mathcal{J} \mathcal{F} g\rangle=$ $\langle f \mid \mathcal{J} \mu(D: \tau) \mathcal{F} g\rangle=\left\langle f \mid \mathcal{F}^{*} \mu(D: \tau) \mathcal{F} g\right\rangle=\left\langle\mu(D: \tau)^{*} \mathcal{F} f \mid \mathcal{F} g\right\rangle$. In view of Theorem 16.16 we see from this that $D f=0$ in the sense of generalized functions if and only if the tempered generalized function $\mu(D: \tau)^{*} \mathcal{F} f$ is perpendicular to $\left[\mathcal{S}\left(i \mathfrak{a}_{\mathrm{q}}^{*}\right) \otimes{ }^{\circ} \mathcal{C}(\tau)\right]^{W}$. This is in turn equivalent to $\mu(D: \tau)^{*} \mathcal{F} f=0$, in view of Lemma 16.1. Since $\mu(D: \tau)$ is invertible almost everywhere the latter assertion is equivalent to $\mathcal{F} f=0$, or $f \in L_{0}^{2}(G / H: \tau)$.

We will say that a function of $L^{2}(G / H)$ belongs to a finite sum of discrete series, if it is contained in a finite sum of irreducible closed invariant subspaces of $L^{2}(G / H)$.

Proposition 17.7 If $\operatorname{dim} \mathfrak{a}_{\mathrm{q}}=1$, then the spaces $\mathcal{C}_{0}(G / H: \tau)$ and $L_{0}^{2}(G / H: \tau)$ are finite dimensional and equal to each other. Any component of a function in these spaces belongs to a finite sum of discrete series. 
Proof. Assume that $\operatorname{dim} \mathfrak{a}_{\mathrm{q}}=1$. We first show that $\mathcal{C}_{0}(G / H: \tau)$ is finite dimensional. Select $D \in \mathbb{D}_{\pi}^{\prime}$ (cf. Lemma 15.3). Then by Proposition 17.3 the space $\mathcal{C}_{0}(G / H: \tau)$ is contained in the kernel $\mathcal{N}$ of $D$ in $C^{\infty}(G / H: \tau)$. Recall the definition of the restriction maps $T_{Q}^{\downarrow}: C^{\infty}(G / H: \tau) \rightarrow C^{\infty}\left(A_{\mathrm{q}}^{+}(Q), V_{\tau}^{K \cap H_{\mathrm{M}}}\right), Q \in \mathcal{P}_{\sigma}^{\min }$, from (47). By the Cartan decomposition (5) the kernels of the maps $T_{Q}^{\downarrow}, Q \in \mathcal{P}_{\sigma}^{\min }$ have a trivial joint intersection. Thus, let $Q \in \mathcal{P}_{\sigma}^{\min }$, then it suffices to show that the space $T_{Q}^{\downarrow}(\mathcal{N})$ has finite dimension. The latter space is contained in the kernel of the radial component $\Pi_{Q, \tau}(D)$ of $D$, defined in (54). Since $\operatorname{dim} A_{\mathrm{q}}=1$, this radial component is an ordinary differential operator. Its coefficients belong to $C^{\infty}\left(A_{\mathrm{q}}^{+}(Q)\right.$, End $\left.\left(V_{\tau}^{K \cap H_{\mathrm{M}}}\right)\right)$. Moreover, by Lemma 15.6 its principal symbol equals $I \otimes X^{k}$, for some $X \in \mathfrak{a}_{\mathrm{q}}, k \in \mathbb{N}$. From this we see that the kernel of $\Pi_{Q, \tau}(D)$, and hence the space $T_{Q}^{\downarrow}(\mathcal{N})$, is a finite dimensional subspace of $C^{\infty}\left(A_{\mathrm{q}}^{+}(Q), V_{\tau}^{K \cap H_{\mathrm{M}}}\right)$. This proves that the space $\mathcal{C}_{0}(G / H: \tau)$ is finite dimensional. It therefore equals its closure in $L^{2}(G / H: \tau)$, which in turn equals $L_{0}^{2}(G / H: \tau)$, by definition.

For the remaining assertion we note that the finite dimensional space $\mathcal{C}_{0}(G / H: \tau)$ is invariant under the action of the center $\mathfrak{Z}$ of $U(\mathfrak{g})$. Thus if $f$ is a component of a function in $\mathcal{C}_{0}(G / H: \tau)$, then $f$ is a $\mathfrak{Z}$ - and $K$-finite function in $\mathcal{C}(G / H)$. By a well known theorem of Harish-Chandra (see e.g. [38], Cor. 3.4.7) the $(\mathfrak{g}, K)$-module $V_{f}$ generated by $f$ is admissible and of finite length (note that it is contained in $\mathcal{C}(G / H)$ ). Its closure $\bar{V}_{f}$ in $L^{2}(G / H)$ is therefore a closed invariant subspace which is admissible and of finite length; by unitarity it decomposes as a finite direct sum of irreducible closed subspaces.

In the following corollary we assume that $G$ is connected, semisimple and linear.

Corollary 17.8 If $\operatorname{dim} \mathfrak{a}_{\mathrm{q}}=1$ and $\operatorname{rank} G / H \neq \operatorname{rank} K / K \cap H$, then we have the inversion formula $\mathcal{J F} f=f$, for all $f \in \mathcal{C}(G / H: \tau)$.

Proof. By Corollary 16.5 and Proposition 17.3 the function $f-\mathcal{J F} f$ belongs to the space $\mathcal{C}_{0}(G / H: \tau)$. The latter is trivial by Proposition 17.7, since the assumption on the rank of $G / H$ implies that there is no discrete series, cf. [35].

\section{The Plancherel decomposition}

In this section we shall describe the Plancherel decomposition of the most-continuous part of $L^{2}(G / H)$. It will be convenient to have the Fourier transform $\hat{f}([\xi]: \lambda)$ defined on classes $[\xi] \in \widehat{M}_{H}$ instead of individual representations $\xi$. For this purpose we fix, once and for all, a distinguished representative $\xi_{\omega}$ for every class $\omega \in \widehat{M}_{H}$. The set of these representatives will be denoted by $\widehat{\mathbf{M}}_{H}$. Let $\omega \in \widehat{M}_{H}$. Then for $f \in C_{c}^{\infty}(G / H), \lambda \in i \mathfrak{a}_{\mathrm{q}}^{*}$ we define

$$
\hat{f}(\omega: \lambda):=\hat{f}\left(\xi_{\omega}: \lambda\right) \in C^{-\infty}\left(K: \xi_{\omega}\right) \otimes V\left(\xi_{\omega}\right)^{*} .
$$

The Weyl group $W$ acts naturally on $\widehat{M}_{H}$ as follows. Let $v \in N_{K}\left(\mathfrak{a}_{\mathrm{q}}\right)$ represent the element $s \in W$, and let $\xi \in \omega \in \widehat{M}_{H}$. Then $s \omega=[v \xi]$, where the representation $v \xi$ 
is defined by $v \xi(m)=\xi\left(v^{-1} m v\right)$. This action on $\widehat{M}_{H}$ transfers to an action on $\widehat{\mathbf{M}}_{H}$ determined by $s \xi_{\omega}=\xi_{s \omega}$

If $\vartheta \subset \widehat{K}$ is a finite set of $K$-types, let $\tau_{\vartheta}$ be defined as in Remark 5.1. Moreover, let $\varsigma$ be the isometry $L^{2}(G / H)_{\vartheta} \rightarrow L^{2}\left(G / H: \tau_{\vartheta}\right)$ defined in $(43)$. We recall that $\varsigma$ maps $C_{c}^{\infty}(G / H)_{\vartheta}$ bijectively onto $C_{c}^{\infty}\left(G / H: \tau_{\vartheta}\right)$.

Proposition 18.1 Let $\vartheta \subset \widehat{K}$ be a finite set of $K$-types, and let $f \in C_{c}^{\infty}(G / H)_{\vartheta}$, $F=\varsigma(f) \in C_{c}^{\infty}\left(G / H: \tau_{\vartheta}\right)$. Then for every $\lambda \in i \mathfrak{a}_{\mathrm{q}}^{*}$ we have:

$$
\|\mathcal{F} F(\lambda)\|^{2}=\sum_{\omega \in \hat{M}_{H}} d_{\omega}\|\hat{f}(\omega: \lambda)\|_{L^{2}\left(K: \xi_{\omega}\right) \otimes V\left(\xi_{\omega}\right)^{*}}^{2}
$$

The sum is finite; $\hat{f}(\omega: \lambda)$ is non-zero only for those $\omega$ for which $\xi_{\omega}^{\vee} \uparrow \tau_{\vartheta}$.

Remark. Notice that $\|\hat{f}(\omega: \lambda)\|$ is independent of the choice of the representative $\xi_{\omega}$.

Proof. This follows readily from (46), since the map $T \mapsto d_{\xi}^{1 / 2} \psi_{T}$ is an isometry.

Corollary 18.2 For every $f \in C_{c}^{\infty}(G / H)$ we have that

$$
\|f\|_{L^{2}(G / H)}^{2} \geq \sum_{\omega \in \widehat{M}_{H}} \int_{i \mathfrak{a}_{\mathrm{q}}^{*}} d_{\omega}\|\hat{f}(\omega: \lambda)\|^{2} d \lambda .
$$

In particular all the integrals on the right-hand side converge, and for almost all $\lambda \in i \mathfrak{a}_{\mathrm{q}}^{*}$ we have $\sum_{\omega} d_{\omega}\|\hat{f}(\omega: \lambda)\|^{2}<\infty$.

Proof. Since the Fourier transform $f \mapsto \hat{f}(\omega: \lambda)$ is equivariant and continuous it suffices to prove this for left $K$-finite $f$. Thus assume that $f \in C_{c}^{\infty}(G / H)_{\vartheta}$, with $\vartheta \subset \widehat{K}$ a finite subset, define $\tau=\tau_{\vartheta}$ as before and let $F=\varsigma(f) \in C_{c}^{\infty}(G / H: \tau)$ (cf. (43)). Applying Corollaries 17.2 and 17.4 we obtain

$$
\|f\|^{2}=\|F\|^{2} \geq\left\|\mathbf{P}_{\mathrm{mc}} F\right\|^{2}=\int_{i_{\mathfrak{a}_{\mathrm{q}}^{*}}}\|\mathcal{F} F(\lambda)\|^{2} d \lambda
$$

Now use Proposition 18.1.

For $\omega \in \widehat{M}_{H}$, we endow

$$
\mathfrak{H}(\omega):=L^{2}\left(K: \xi_{\omega}\right) \otimes V\left(\xi_{\omega}\right)^{*}
$$

with the tensor product Hilbert structure. Let the algebraic direct sum

$$
\mathfrak{H}_{\mathrm{alg}}=\bigoplus_{\omega \in \widehat{M}_{H}} \mathfrak{H}(\omega)
$$


be equipped with the inner product defined by

$$
\langle v \mid w\rangle=\sum_{\omega \in \widehat{M}_{H}} d_{\omega}\left\langle v_{\omega} \mid w_{\omega}\right\rangle \quad\left(v, w \in \mathfrak{H}_{\mathrm{alg}}\right) .
$$

Moreover, let $\mathfrak{H}$ be the Hilbert completion of $\mathfrak{H}_{\text {alg }}$ for this inner product, and let $\mathfrak{L}^{2}$ denote the Hilbert space of square integrable functions $i \mathfrak{a}_{\mathrm{q}}^{*} \rightarrow \mathfrak{H}$. If $\varphi \in \mathfrak{L}^{2}$, we write $\varphi(\omega: \lambda)=\varphi(\lambda)_{\omega}$. With these notations we have

$$
\|\varphi\|_{\mathfrak{L}^{2}}^{2}=\sum_{\omega \in \hat{M}_{H}} \int_{i \mathfrak{a}_{\mathrm{q}}^{*}} d_{\omega}\|\varphi(\omega: \lambda)\|^{2} d \lambda .
$$

Thus, if $f \in C_{c}^{\infty}(G / H)$, then by Corollary 18.2 we have that $\hat{f}(\lambda) \in \mathfrak{H}$ for almost all $\lambda \in i \mathfrak{a}_{\mathrm{q}}^{*}$, and that $\hat{f} \in \mathfrak{L}^{2}$ with

$$
\|\hat{f}\|_{\mathfrak{L}^{2}} \leq\|f\|_{L^{2}(G / H)}
$$

There is a natural unitary representation $\pi=\pi_{P}$ of $G$ in $\mathfrak{L}^{2}$, given by:

$$
(\pi(x) \varphi)(\omega: \lambda)=\left[\pi_{P, \xi_{\omega},-\lambda}(x) \otimes I\right] \varphi(\omega: \lambda) \quad(x \in G) .
$$

Lemma 4.3 implies that the map $f \mapsto \hat{f}$ intertwines the regular action $L$ of $G$ on $C_{c}^{\infty}(G / H)$ with this representation $\pi$. The following result is now immediate.

Proposition 18.3 The map $f \mapsto \hat{f}$ has a unique extension to a $G$-equivariant continuous linear map $\mathfrak{F}$ from $\left(L, L^{2}(G / H)\right)$ to $\left(\pi, \mathfrak{L}^{2}\right)$.

Now let $L_{0}^{2}(G / H)=\operatorname{ker} \mathfrak{F}$ and let $L_{\mathrm{mc}}^{2}(G / H)$ be its orthocomplement in $L^{2}(G / H)$. Then

$$
L^{2}(G / H)=L_{0}^{2}(G / H) \oplus L_{\mathrm{mc}}^{2}(G / H)
$$

is an orthogonal direct sum decomposition into closed $G$-invariant subspaces.

Proposition 18.4 The restriction of $\mathfrak{F}$ to $L_{\mathrm{mc}}^{2}(G / H)$ is an isometry.

Proof. Let $\vartheta \subset \widehat{K}$ be a finite subset. Then it suffices to show that the restriction of $\mathfrak{F}$ to $L_{\mathrm{mc}}^{2}(G / H)_{\vartheta}$ is an isometry. The following lemma allows us to do this by reduction to Corollary 17.4 .

Let $\vartheta \subset \widehat{K}$ be a finite subset and put $\tau=\tau_{\vartheta}$.

Lemma 18.5 For $f \in L^{2}(G / H)_{\vartheta}$ and $F=\varsigma(f)$ we have

$$
\|\mathfrak{F} f\|_{\mathfrak{L}^{2}}=\|\mathcal{F} F\| \text {. }
$$

Moreover, the map $\varsigma$ restricts to isometries

$$
L_{0}^{2}(G / H)_{\vartheta} \stackrel{\simeq}{\longrightarrow} L_{0}^{2}(G / H: \tau) \quad \text { and } \quad L_{\mathrm{mc}}^{2}(G / H)_{\vartheta} \stackrel{\simeq}{\longrightarrow} L_{\mathrm{mc}}^{2}(G / H: \tau) .
$$

Proof. It follows from Proposition 18.1 that (156) holds for $f \in C_{c}^{\infty}(G / H)_{\vartheta}$. By density and continuity this result is still true for $f \in L^{2}(G / H)_{\vartheta}$. From this we see that $L_{0}^{2}(G / H: \tau)=\operatorname{ker} \mathcal{F}=\varsigma\left(L_{0}^{2}(G / H)_{\vartheta}\right)$, and taking orthocomplements we conclude that $L_{\mathrm{mc}}^{2}(G / H: \tau)=\varsigma\left(L_{\mathrm{mc}}^{2}(G / H)_{\vartheta}\right)$. 
To find the explicit direct integral decomposition of $L_{\mathrm{mc}}^{2}(G / H)$, we must characterize the image of $\mathfrak{F}$. For this we need to define a representation, of $W$ on $\mathfrak{L}^{2}$ which is the analogue of the representation $\gamma$ defined in (142).

If $\mathcal{H}$ is a Hilbert space, let $\operatorname{End}(\mathcal{H})$ denote the Banach space of continuous linear endomorphisms of $\mathcal{H}$, equipped with the operator norm.

Proposition 18.6 For each $s \in W$ there exists a measurable map $\mathfrak{C}_{s}: i \mathfrak{a}_{\mathbf{q}}^{*} \rightarrow \operatorname{End}(\mathfrak{H})$, which is almost everywhere uniquely determined, such that $\lambda \mapsto\left\|\mathfrak{C}_{s}(\lambda)\right\|$ is bounded, and such that for every $f \in C_{c}^{\infty}(G / H)$ we have

$$
\hat{f}(s \lambda)=\mathfrak{C}_{s}(\lambda) \hat{f}(\lambda)
$$

for almost all $\lambda \in i \mathfrak{a}_{\mathrm{q}}^{*}$. For almost all $\lambda \in i \mathfrak{a}_{\mathrm{q}}^{*}$ the map $\mathfrak{C}_{s}(\lambda): \mathfrak{H} \rightarrow \mathfrak{H}$ is unitary, and restricts to a unitary operator $\mathfrak{C}_{s}(\omega: \lambda): \mathfrak{H}(\omega) \rightarrow \mathfrak{H}(s \omega)$, intertwining $\pi_{\xi_{\omega},-\lambda}$ with $\pi_{\xi_{s \omega},-s \lambda}$, for every $\omega \in \widehat{M}_{H}$. Moreover, for all $s, t \in W$ we have:

$$
\mathfrak{C}_{s t}(\omega: \lambda)=\mathfrak{C}_{s}(t \omega: t \lambda) \circ \mathfrak{C}_{t}(\omega: \lambda)
$$

In particular $\mathfrak{C}_{1}(\lambda)=I$ and $\mathfrak{C}_{s}(\lambda)^{-1}=\mathfrak{C}_{s^{-1}}(s \lambda)$ for all $s \in W$.

Before giving the proof of this proposition we note the following consequence. For $s \in W$, let $\mathfrak{C}_{s}$ be as above and define a map , $(s): \mathfrak{L}^{2} \rightarrow \mathfrak{L}^{2}$ by

$$
[,(s) \varphi](\lambda)=\mathfrak{C}_{s^{-1}}(\lambda)^{-1} \varphi\left(s^{-1} \lambda\right)=\mathfrak{C}_{s}\left(s^{-1} \lambda\right) \varphi\left(s^{-1} \lambda\right) \quad\left(\varphi \in \mathfrak{L}^{2}\right) .
$$

Then it follows that, $(s)$ is $\pi(G)$-equivariant, and that, $: s \mapsto,(s)$ defines a unitary representation of $W$ in $\mathfrak{L}^{2}$, satisfying

$$
(,(s) \hat{f})(\lambda)=\hat{f}(\lambda)
$$

for $f \in C_{c}^{\infty}(G / H)$. A different characterization of , in terms of standard intertwining operators will be given in Corollary 19.6.

Turning to the proof of the above proposition we will first establish uniqueness. If $\Omega \subset i \mathfrak{a}_{\mathrm{q}}^{*}$ is a measurable subset, we write $\mathfrak{L}_{\Omega}^{2}$ for the closed invariant subspace of $\mathfrak{L}^{2}$ consisting of elements that vanish outside $\Omega$. If $\varphi \in \mathfrak{L}^{2}$, let $\varphi_{\Omega}$ be the unique element of $\mathfrak{L}_{\Omega}^{2}$ that equals $\varphi$ on $\Omega$. Then $\varphi \mapsto \varphi_{\Omega}$ is an orthogonal projection. Since multiplication by a bounded measurable map is $L^{2}$-continuous, the uniqueness statement of Proposition 18.6 is an immediate consequence of the following lemma.

Lemma 18.7 Let $\Omega$ be an open chamber in $i \mathfrak{a}_{\mathrm{q}}^{*}$ with respect to $\Sigma$. Then $f \mapsto \hat{f}_{\Omega}$ maps $C_{c}^{\infty}(G / H)$ onto a dense subspace of $\mathfrak{L}_{\Omega}^{2}$, and $C_{c}^{\infty}(G / H)_{\vartheta}$ onto a dense subspace of $\left(\mathfrak{L}_{\Omega}^{2}\right)_{\vartheta}$, for all finite sets $\vartheta \subset \widehat{K}$.

Proof. By density of the algebraic sum of the $\left(\mathfrak{L}_{\Omega}^{2}\right)_{\vartheta}$ in $\mathfrak{L}_{\Omega}^{2}$ it suffices to prove the statement about $C_{c}^{\infty}(G / H)_{\vartheta}$ for all $\vartheta$. Let $T \in\left(\mathfrak{L}_{\Omega}^{2}\right)_{\vartheta}$, and suppose that

$$
\langle\hat{f} \mid T\rangle=0 \quad \text { for all } \quad f \in C_{c}^{\infty}(G / H)_{\vartheta}
$$


Then we must show that $T=0$. Put $T(\lambda)=\sum_{\omega} T(\omega: \lambda)$, with $T(\omega: \lambda) \in \mathfrak{H}(\omega)_{\vartheta}=$ $L^{2}\left(K: \xi_{\omega}\right)_{\vartheta} \otimes V\left(\xi_{\omega}\right)^{*}$. Notice that the sum is finite. Let $\tau=\tau_{\vartheta}$ be as before. Then $\psi_{T(\omega: \lambda)} \in{ }^{\circ} \mathcal{C}_{\omega}(\tau)$. Define $\Psi: i \mathfrak{a}_{\mathrm{q}}^{*} \rightarrow{ }^{\circ} \mathcal{C}(\tau)$ by

$$
\Psi(\lambda)=\sum_{\omega \in \widehat{M}_{H}} d_{\omega} \psi_{T(\omega: \lambda)}
$$

Then $\Psi \in L^{2}\left(i \mathfrak{a}_{\mathrm{q}}^{*}\right) \otimes{ }^{\circ} \mathcal{C}(\tau)$, and $\Psi=0$ outside $\Omega$. Let now $F \in C_{c}^{\infty}(G / H: \tau)$, and put $f=\varsigma^{-1} F \in C_{c}^{\infty}(G / H)_{\vartheta}$. Then by Proposition 6.4 we have

$$
\langle\mathcal{F} F \mid \Psi\rangle=\sum_{\omega \in \widehat{M}_{H}} \int_{i \mathfrak{a}_{\mathrm{q}}^{*}} d_{\omega}\langle\hat{f}(\omega: \lambda) \mid T(\omega: \lambda)\rangle d \lambda=\langle\hat{f} \mid T\rangle=0 .
$$

Since the image of $C_{c}^{\infty}(G / H: \tau)$ under $\mathcal{F}$ is dense in $\left(L^{2}\left(i \mathfrak{a}_{\mathrm{q}}^{*}\right) \otimes{ }^{\circ} \mathcal{C}(\tau)\right)^{W}$ by Theorem 16.16, it follows that $\Psi$ is perpendicular to $\left(L^{2}\left(i \mathfrak{a}_{\mathrm{q}}^{*}\right) \otimes{ }^{\circ} \mathcal{C}(\tau)\right)^{W}$. In combination with the fact that $\Psi$ vanishes outside a fundamental domain for the action of $W$, this implies that $\Psi=0$, and we conclude that $T=0$.

Proof of Proposition 18.6. Let $s \in W$. Fix a finite subset $\vartheta \subset \widehat{K}$ and let $\tau=\tau_{\vartheta}$ be as before. For $\omega \in \widehat{M}_{H}$, recall (Lemma 5.6) that the map $\mathfrak{H}(\omega)_{\vartheta} \rightarrow{ }^{\circ} \mathcal{C}_{\omega}(\tau), T \mapsto d_{\omega}^{1 / 2} \psi_{T}$ is an isometry. For $\lambda \in i \mathfrak{a}_{\mathrm{q}}^{*}$ we notice that the endomorphism $C_{P \mid P}^{\circ}(s: \lambda)$ of ${ }^{\circ} \mathcal{C}(\tau)$ maps ${ }^{\circ} \mathcal{C}_{\omega}(\tau)$ into ${ }^{\circ} \mathcal{C}_{s \omega}(\tau)$ (cf. [13], eqn. (68)) and define the map

$$
\mathfrak{C}_{s}(\omega: \lambda)_{\vartheta}: \mathfrak{H}(\omega)_{\vartheta} \rightarrow \mathfrak{H}(s \omega)_{\vartheta}
$$

by

$$
\psi_{\mathfrak{c}_{s}(\omega: \lambda) T}=C_{P \mid P}^{\circ}(s: \lambda) \psi_{T} \quad\left(T \in \mathfrak{H}(\omega)_{\vartheta}\right) .
$$

Then it follows from Lemma 5.6 and Proposition 5.3 that $\mathfrak{C}_{s}(\omega: \lambda)_{\vartheta}$ is a unitary map. For $\lambda \in i \mathfrak{a}_{\mathbf{q}}^{*}$ we define the unitary map $\mathfrak{C}_{s}(\lambda)_{\vartheta} \in \operatorname{End}\left(\mathfrak{H}_{\vartheta}\right)$ by $\mathfrak{C}_{s}(\lambda)_{\vartheta}=\mathfrak{C}_{s}(\omega: \lambda)_{\vartheta}$ on $\mathfrak{H}(\omega)_{\vartheta}$. Notice that the map $\lambda \mapsto \mathfrak{C}_{s}(\lambda)_{\vartheta}$ is continuous since $\lambda \mapsto C_{P \mid P}^{\circ}(s: \lambda)$ is continuous. Let now $f \in C_{c}^{\infty}(G / H)_{\vartheta}$, and let $F=\varsigma f \in C_{c}^{\infty}(G / H: \tau)$. Then using Lemma 6.3, Proposition 6.4 and (161) we easily see that

$$
\hat{f}(s \lambda)=\mathfrak{C}_{s}(\lambda)_{\vartheta} \hat{f}(\lambda) \quad\left(f \in C_{c}^{\infty}(G / H)_{\vartheta}\right) .
$$

Now let $\vartheta^{\prime} \subset \widehat{K}$ be a finite subset containing $\vartheta$. Then it follows by (162) and Lemma 18.7 together with the continuity of $\lambda \mapsto \mathfrak{C}_{s}(\lambda)_{\vartheta}$ that $\mathfrak{C}_{s}(\lambda)_{\vartheta}$ restricts to $\mathfrak{C}_{s}(\lambda)_{\vartheta}$ on $\mathfrak{H}_{\vartheta}$ for all $\lambda \in i \mathfrak{a}_{\mathrm{q}}^{*}$. Hence for every $\lambda \in i \mathfrak{a}_{\mathrm{q}}^{*}$ there exists a unique unitary map $\mathfrak{C}_{s}(\lambda): \mathfrak{H} \rightarrow \mathfrak{H}$ such that $\mathfrak{C}_{s}(\lambda)=\mathfrak{C}_{s}(\lambda)_{\vartheta}$ on $\mathfrak{H}_{\vartheta}$, for all $\vartheta$. The map $\lambda \mapsto \mathfrak{C}_{s}(\lambda)$ is measurable, since it is the limit of a sequence of continuous maps. Moreover, from (162) it follows that $\mathfrak{C}_{s}$ satisfies (157) for $K$-finite functions $f \in C_{c}^{\infty}(G / H)$, for all $\lambda \in i \mathfrak{a}_{\mathrm{q}}^{*}$. By density and continuity (Proposition 18.3) it follows that (157) holds for all $f$ as an identity in $\mathfrak{L}^{2}$, hence also pointwise almost everywhere. The existence of $\mathfrak{C}_{s}$ is now established; the uniqueness was obtained earlier. The remaining assertions are straightforward consequences of the construction above. 
We can now describe the image of $\mathfrak{F}$. Let $\left(\mathfrak{L}^{2}\right)^{W}$ denote the set of $W$-invariants in $\mathfrak{L}^{2}$ for the representation, , defined by (158). This is an invariant subspace for $\pi$, since, $(s)$ is equivariant for each $s \in W$.

Theorem 18.8 The Fourier transform $\mathfrak{F}$ is an equivariant isometry from $L_{\mathrm{mc}}^{2}(G / H)$ onto $\left(\mathfrak{L}^{2}\right)^{W}$.

Proof. Let $U$ denote the image of $C_{c}^{\infty}(G / H)_{K}$ under $\mathfrak{F}$. Then $U \subset\left(\mathfrak{L}^{2}\right)^{W}$ by $(159)$. The map $\mathfrak{F}$ being an isometry, it suffices to show that $U$ is dense in $\left(\mathfrak{L}^{2}\right)^{W}$.

Let $\Omega$ be an open chamber in $i \mathfrak{a}_{\mathrm{q}}^{*}$. If $\varphi \in \mathfrak{L}^{2}$, recall that we write $\varphi_{\Omega}$ for the restriction of $\varphi$ to $\Omega$, i.e. the unique element of $\mathfrak{L}_{\Omega}^{2}$ satisfying $\varphi_{\Omega}=\varphi$ on $\Omega$. One readily verifies that $|W|^{1 / 2}$ times the map

$$
\Phi:\left(\mathfrak{L}^{2}\right)^{W} \rightarrow \mathfrak{L}_{\Omega}^{2}, \varphi \mapsto \varphi_{\Omega}
$$

is an equivariant isometry, and therefore it suffices to show that $\Phi(U)$ is dense in $\mathfrak{L}_{\Omega}^{2}$. Now this follows from Lemma 18.7.

Fix a choice $\Sigma^{+}$of positive roots for the root system $\Sigma=\Sigma\left(\mathfrak{g}, \mathfrak{a}_{\mathrm{q}}\right)$, and let $\mathfrak{a}_{\mathrm{q}}^{*+}$ be the positive Weyl chamber in $\mathfrak{a}_{\mathrm{q}}^{*}$.

Theorem 18.9 The map $\mathfrak{F}$ induces the following Plancherel decomposition of the restriction of the regular representation $L$ to $L_{\mathrm{mc}}^{2}(G / H)$ :

$$
\left.L\right|_{L_{\mathrm{mc}}^{2}(G / H)} \simeq \sum_{\omega \in \widehat{M}_{H}} \int_{i_{\mathfrak{a}_{\mathrm{q}}^{*+}}}^{\oplus} V\left(\xi_{\omega}\right)^{*} \otimes \pi_{\xi_{\omega}, \lambda} \quad d_{\omega}|W| d \lambda,
$$

and

$$
\|f\|^{2}=|W| \sum_{\omega \in \widehat{M}_{H}} d_{\omega} \int_{i \mathfrak{a}_{\mathrm{q}}^{*+}}^{\oplus}\|\mathfrak{F} f(\omega: \lambda)\|^{2} d \lambda
$$

for $f \in L_{\mathrm{mc}}^{2}(G / H)$. In particular, for every $\omega \in \widehat{M}_{H}$ the principal series $\pi_{\xi_{\omega}, \lambda}$ occurs with multiplicity $m_{\omega}=\operatorname{dim} V\left(\xi_{\omega}\right)$ for almost every $\lambda \in i \mathfrak{a}_{\mathbf{q}}^{*+}$.

Remark 18.10 For further results on the multiplicities in the decomposition (164) we refer to [11].

Proof. Put $\Omega=-i \mathfrak{a}_{\mathrm{q}}^{*+}$, and let $\Phi$ be defined as in (163). The map $\Phi \circ \mathfrak{F}$ now induces the direct integral decomposition (164). If $\omega \in \widehat{M}_{H}$, then for every $\lambda \in i \mathfrak{a}_{\mathrm{q}}^{*+}$ the principal series representation $\pi_{\xi_{\omega}, \lambda}$ is irreducible (cf. [6], Prop. 3.7). Moreover, owing to the restriction on the domain of $\lambda$, there is no double occurrence of the representations $\pi_{\xi_{\omega}, \lambda}$. To be more precise, let $\omega, \omega^{\prime} \in \widehat{M}_{H}$ be fixed. Then for almost all $\lambda, \lambda^{\prime} \in i \mathfrak{a}_{\mathrm{q}}^{*+}$ the representations $\pi_{\xi_{\omega}, \lambda}$ and $\pi_{\xi_{\omega^{\prime}}, \lambda^{\prime}}$ are equivalent if and only if $\left(\omega^{\prime}, \lambda^{\prime}\right)=(s \omega, s \lambda)$ for some $s \in W$. Since $\lambda$ and $\lambda^{\prime}$ are in the same chamber, the latter condition is in turn equivalent to $\left(\omega^{\prime}, \lambda^{\prime}\right)=(\omega, \lambda)$.

These final remarks imply that indeed (164) establishes the Plancherel decomposition, with multiplicities as described in the final assertion of the theorem. 
The following result expresses that the orthocomplement $L_{0}^{2}(G / H)$ of $L_{\mathrm{mc}}^{2}(G / H)$ is small in a certain spectral sense. Recall the definition of $\mathbb{D}_{\tau, \pi}$ from Definition 10.3 and let $\mathbb{D}_{\vartheta, \pi}=\mathbb{D}_{\tau_{\vartheta}, \pi}$.

\section{Theorem 18.11}

(a) Let $\vartheta \subset \widehat{K}$ be a finite subset. Then for any $D \in \mathbb{D}_{\vartheta, \pi}$ we have (in the sense of generalized functions):

$$
L_{0}^{2}(G / H)_{\vartheta}=L^{2}(G / H)_{\vartheta} \cap \operatorname{ker} D
$$

(b) $L_{0}^{2}(G / H) \cap C_{c}^{\infty}(G / H)=0$.

(c) If $\operatorname{dim} \mathfrak{a}_{\mathrm{q}}=1$, then the space $L_{0}^{2}(G / H)$ decomposes discretely.

Proof. (a) Let $\tau=\tau_{\vartheta}$. Let $f \in L_{0}^{2}(G / H)_{\vartheta}$, and put $F=\varsigma f$. Then $F \in L^{2}(G / H: \tau)$ and $f=\delta_{e} \circ F$. Now $D f=0$ as a generalized function if and only if $D F=0$ as a generalized function. Therefore the result follows by application of Lemma 18.5 and Lemma 17.6.

(b) This is equivalent to Theorem 15.5.

(c) Assume that $\operatorname{dim} \mathfrak{a}_{\mathrm{q}}=1$. Let $\vartheta \subset \widehat{K}$ be any finite subset and put $\tau=\tau_{\vartheta}$. Then using the first isometry of Lemma 18.5 we infer from Proposition 17.7 that the space $L_{0}^{2}(G / H)_{\vartheta}$ is finite dimensional. Moreover, its elements are components of functions in $L_{0}^{2}(G / H: \tau)$, and by again applying Proposition 17.7 we see that $L_{0}^{2}(G / H)_{\vartheta}$ is contained in the discrete part $L_{d}^{2}(G / H)$ (that is, the closure of the span of all the irreducible closed invariant subspaces) of $L^{2}(G / H)$. Since $\vartheta$ was arbitrary we infer that $L_{0}^{2}(G / H)$ is contained in $L_{d}^{2}(G / H)$, hence it decomposes discretely.

\section{Dependence on choices}

In this section we shall discuss the dependence of the Plancherel formula for $L_{\mathrm{mc}}^{2}(G / H)$ on the choices made. We will also derive a formula for the representation, of $W$ in $\mathfrak{L}^{2}$ in terms of intertwining operators.

We first discuss the dependence of the Plancherel formula on the choice $\mathcal{W}$ of representatives for $W / W_{K \cap H}$. Let $\mathcal{W}^{\prime}$ be a second choice of representatives. Let $V^{\prime}(\xi)([\xi] \in$ $\left.\widehat{M}_{H}\right), j^{\prime}, j^{\prime \prime}, \hat{f}_{P}^{\prime}, \mathfrak{F}^{\prime}$ and $\mathfrak{L}^{2}, \pi^{\prime}$ be defined as before, but with $\mathcal{W}$ replaced by $\mathcal{W}^{\prime}$. Then according to $[6]$, Lemma 5.8 , there exists, for every $[\xi] \in \widehat{M}_{H}$, a unique unitary map $R(\xi): V(\xi) \rightarrow V^{\prime}(\xi)$ such that

$$
j^{\prime}(P: \xi: \lambda) \circ R(\xi)=j(P: \xi: \lambda),
$$

for $P \in \mathcal{P}_{\sigma}^{\min }, \lambda \in \mathfrak{a}_{\mathrm{qc}}^{*}$. Since the map $R(\xi)$ does not depend on $P \in \mathcal{P}_{\sigma}^{\text {min }}$, it follows from the definition of $j^{\circ}$ (cf. (24)) that (166) holds with $j$ replaced by $j^{\circ}$. Hence

$$
\hat{f}_{P}^{\prime}(\xi: \lambda)=\left[I \otimes R(\xi)^{-1 t}\right] \hat{f}_{P}(\xi: \lambda) \in L^{2}(K: \xi) \otimes V^{\prime}(\xi)^{*}
$$


for all $f \in C_{c}^{\infty}(G / H), \lambda \in i \mathfrak{a}_{\mathrm{q}}^{*}$. Let the map $\mathfrak{R}: \mathfrak{L}^{2} \rightarrow \mathfrak{L}^{2}$ be defined by

$$
\mathfrak{R} \varphi(\omega: \lambda)=\left[I \otimes R\left(\xi_{\omega}\right)^{-1 t}\right] \varphi(\omega: \lambda) \quad\left(\varphi \in \mathfrak{L}^{2}, \omega \in \widehat{M}_{H}, \lambda \in i \mathfrak{a}_{\mathrm{q}}^{*}\right) .
$$

Then the following result expresses the dependence of the decomposition (164) on $\mathcal{W}$.

Lemma 19.1 The map $\mathfrak{R}: \mathfrak{L}^{2} \rightarrow \mathfrak{L}^{2}$ is a unitary isomorphism, intertwining $\pi$ and $\pi^{\prime}$. Moreover, $\mathfrak{F}^{\prime}=\mathfrak{R} \circ \mathfrak{F}$.

We now turn to the dependence of the Plancherel decomposition on the choice of the parabolic subgroup $P \in \mathcal{P}_{\sigma}^{\min }$. If $P, Q \in \mathcal{P}_{\sigma}^{\min },[\xi] \in \widehat{M}_{\mathrm{fu}}, \lambda \in \mathfrak{a}_{\mathrm{qc}}^{*}$, let the standard intertwining operator $A(Q: P: \xi: \lambda)$ from $\pi_{P, \xi, \lambda}$ to $\pi_{Q, \xi, \lambda}$ be defined as in $[6]$ (see also the text preceding (24)).

We recall from [6], Prop. 6.1, that there exists a unique meromorphic End $(V(\xi))$-valued meromorphic function $B(Q: P: \xi)$ on $\mathfrak{a}_{\mathrm{q} c}^{*}$, such that

$$
A(Q: P: \xi: \lambda) \circ j(P: \xi: \lambda)=j(Q: \xi: \lambda) \circ B(Q: P: \xi: \lambda)
$$

as a meromorphic identity in $\lambda \in \mathfrak{a}_{\mathrm{qc}}^{*}$. The version of this transformation rule for $j^{\circ}$ is given by the following lemma.

Lemma 19.2 Let $P, Q \in \mathcal{P}_{\sigma}^{\min }$. Then for every $[\xi] \in \widehat{M}_{H}$ we have

$$
A(Q: P: \xi: \lambda) \circ j^{\circ}(P: \xi: \lambda)=j^{\circ}(Q: \xi: \lambda) \circ B(\bar{Q}: \bar{P}: \xi: \lambda) \text {. }
$$

Proof. See [13], diagram (14).

Let $P, Q \in \mathcal{P}_{\sigma}^{\text {min }}$. Then it follows from the above that for all $f \in C_{c}^{\infty}(G / H),[\xi] \in \widehat{M}_{H}$ and $\lambda \in i \mathfrak{a}_{\mathrm{q}}^{*}$ we have:

$$
\hat{f}_{Q}(\xi: \lambda)=\left[A(Q: P: \xi: \lambda) \otimes B(\bar{Q}: \bar{P}: \xi: \lambda)^{-1 t}\right] \hat{f}_{P}(\xi: \lambda) .
$$

We now define the map $\mathfrak{A}(Q: P): \mathfrak{L}^{2} \rightarrow \mathfrak{L}^{2}$ by

$$
\mathfrak{A}(Q: P) \varphi(\omega: \lambda)=\left[A\left(Q: P: \xi_{\omega}: \lambda\right) \otimes B\left(\bar{Q}: \bar{P}: \xi_{\omega}: \lambda\right)^{-1 t}\right] \varphi(\omega: \lambda),
$$

for $\varphi \in \mathfrak{L}^{2}, \omega \in \widehat{M}_{H}, \lambda \in i \mathfrak{a}_{\mathrm{q}}^{*}$. The following result describes the dependence of the decomposition in Theorem 18.9 on the parabolic subgroup $P$.

Lemma 19.3 The map $\mathfrak{A}(Q: P)$ is an equivariant unitary isomorphism from $\left(\mathfrak{L}^{2}, \pi_{P}\right)$ onto $\left(\mathfrak{L}^{2}, \pi_{Q}\right)$. Moreover, $\mathfrak{F}_{Q}=\mathfrak{A}(Q: P) \circ \mathfrak{F}_{P}$.

Proof. According to [7], 115 , we have, for $\omega \in \widehat{M}_{H}, \lambda \in i \mathfrak{a}_{\mathrm{q}}^{*}$, the identities

$$
\begin{aligned}
& A\left(Q: P: \xi_{\omega}: \lambda\right)^{*} A\left(Q: P: \xi_{\omega}: \lambda\right)=\eta\left(Q: P: \xi_{\omega}: \lambda\right) I, \\
& B\left(\bar{Q}: \bar{P}: \xi_{\omega}: \lambda\right)^{*} B\left(\bar{Q}: \bar{P}: \xi_{\omega}: \lambda\right)=\eta\left(\bar{Q}: \bar{P}: \xi_{\omega}: \lambda\right) I .
\end{aligned}
$$

Moreover, combining Lemma 15.8 and equation (129) of [7] we obtain a third identity $\eta\left(Q: P: \xi_{\omega}: \lambda\right)=\eta\left(\bar{Q}: \bar{P}: \xi_{\omega}: \lambda\right)$. The unitarity of $\mathfrak{A}(Q: P)$ follows from these three identities. The proof of the other assertions is straightforward. 
Next we investigate the dependence of the Plancherel decomposition on the choice of the representatives $\xi_{\omega}$. First, let $\omega$ be a class in $\widehat{M}_{H}$, and suppose that $\xi, \xi^{\prime} \in \omega$. Fix a unitary intertwining operator $T: \mathcal{H}_{\xi} \rightarrow \mathcal{H}_{\xi^{\prime}}$. Then $T$ naturally induces the map $I(T): C^{-\infty}(P: \xi: \lambda) \rightarrow C^{-\infty}\left(P: \xi^{\prime}: \lambda\right)$ given by $f \mapsto T \circ f$. Recall that the space $V(\xi)$ is the formal direct sum of the spaces $V(\xi, w)$, with $w$ ranging over $\mathcal{W}$. For $w \in \mathcal{W}$, let $i(T, w): V(\xi, w) \rightarrow V\left(\xi^{\prime}, w\right)$ be the natural map induced by $T$, and let $i(T): V(\xi) \rightarrow V\left(\xi^{\prime}\right)$ be the direct sum of the maps $i(T, w)$. Then it is obvious from the definitions that

$$
I(T) \circ j^{\circ}(P: \xi: \lambda)=j^{\circ}\left(P: \xi^{\prime}: \lambda\right) \circ i(T) .
$$

One readily verifies that the restriction of $I(T) \otimes i(T)^{-1 t}$ to a map $L^{2}(K: \xi) \otimes V(\xi)^{*} \rightarrow$ $L^{2}\left(K: \xi^{\prime}\right) \otimes V\left(\xi^{\prime}\right)^{*}$ does not depend on the particular choice of $T$. Therefore we denote this restriction by $I\left(\xi^{\prime}, \xi\right)$. It is obviously unitary, and it intertwines the representations $\pi_{P, \xi, \lambda} \otimes I$ and $\pi_{P, \xi^{\prime}, \lambda} \otimes I$. Moreover, it follows from the above that for every $f \in C_{c}^{\infty}(G / H)$ and $\lambda \in i \mathfrak{a}_{\mathrm{q}}^{*}$ we have:

$$
\hat{f}\left(\xi^{\prime}: \lambda\right)=I\left(\xi^{\prime}, \xi\right) \hat{f}(\xi: \lambda) .
$$

Now fix for every $\omega \in \widehat{M}_{H}$ a second representative $\xi_{\omega}^{\prime}$. Let $\widehat{\mathbf{M}}_{H}^{\prime}$ denote the set of these representatives, and define $\mathfrak{H}^{\prime}, \mathfrak{L}^{2}, \pi^{\prime}$ and $\mathfrak{F}^{\prime}$ as in Section 18 but with everywhere $\xi_{\omega}^{\prime}$ instead of $\xi_{\omega}$, for $\omega \in \widehat{M}_{H}$. Let $I\left(\widehat{\mathbf{M}}_{H}^{\prime}, \widehat{\mathbf{M}}_{H}\right)$ denote the direct sum of the maps $I\left(\xi_{\omega}^{\prime}, \xi_{\omega}\right): \mathfrak{H}(\omega) \rightarrow \mathfrak{H}^{\prime}(\omega)$, and let $\mathfrak{I}\left(\widehat{\mathbf{M}}_{H}^{\prime}, \widehat{\mathbf{M}}_{H}\right): \mathfrak{L}^{2} \rightarrow \mathfrak{L}^{2}$ be defined by $\varphi \mapsto$ $I\left(\widehat{\mathbf{M}}_{H}^{\prime}, \widehat{\mathbf{M}}_{H}\right) \circ \varphi$. The following straightforward result now describes the dependence of the Plancherel decomposition on the choice of $\widehat{\mathbf{M}}_{H}$.

Lemma 19.4 The map $\mathfrak{I}\left(\widehat{\mathbf{M}}_{H}^{\prime}, \widehat{\mathbf{M}}_{H}\right): \mathfrak{L}^{2} \rightarrow \mathfrak{L}^{2}$ is an equivariant unitary isomorphism. Moreover, $\mathfrak{F}^{\prime}=\mathfrak{I}\left(\widehat{\mathbf{M}}_{H}^{\prime}, \widehat{\mathbf{M}}_{H}\right) \circ \mathfrak{F}$.

We will finally use the material of this section to give a characterization of the representation, of $W$ in $\mathfrak{L}^{2}$, different from the one in Proposition 18.6. For this we need transformation properties of $j^{\circ}$ under the action of the Weyl group. For $v \in N_{K}\left(\mathfrak{a}_{\mathrm{q}}\right)$, let $L(v): C^{-\infty}(P: \xi: \lambda) \rightarrow C^{-\infty}\left(v P v^{-1}: v \xi: v \lambda\right)$ be the intertwining operator defined by $L(v) \varphi(x)=\varphi\left(v^{-1} x\right)$. Then by [6], Lemma 6.10, there exists a unique unitary map $L(\xi, v): V(\xi) \rightarrow V(v \xi)$, not depending on $P, \lambda$, such that

$$
L(v) \circ j(P: \xi \circ \lambda)=j\left(v P v^{-1}: v \xi: v \lambda\right) \circ L(\xi, v) .
$$

By [13], eqn. (63), we have

$$
\hat{f}_{v v^{-1}}(v \xi: v \lambda)=\left[L(v) \otimes L(\xi, v)^{-1 t}\right] \hat{f}_{P}(\xi: \lambda)
$$

for all $f \in C_{c}^{\infty}(G / H),[\xi] \in \widehat{M}_{H}, \lambda \in i \mathfrak{a}_{\mathrm{q}}^{*}$.

Now fix $v \in N_{K}\left(\mathfrak{a}_{q}\right)$, and let $s$ denote its image in $W$. Put $\widehat{\mathbf{M}}_{H}^{\prime}=v \widehat{\mathbf{M}}_{H}$, so that $\xi_{\omega}^{\prime}=v \xi_{s^{-1} \omega}$, and let $\mathfrak{H}^{\prime}, \mathfrak{L}^{2}, \pi^{\prime}$ and $\mathfrak{F}^{\prime}$ be defined as above. Define the map $m(v): \mathfrak{L}^{2} \rightarrow \mathfrak{L}^{2}$ by

$$
[m(v) \varphi](\omega: \lambda)=\left[L(v) \otimes L\left(\xi_{s^{-1} \omega}, v\right)^{-1 t}\right] \varphi\left(s^{-1} \omega: s^{-1} \lambda\right)
$$


Then $m(v)$ is a unitary equivariant isomorphism $\left(\mathfrak{L}^{2}, \pi_{P}\right) \rightarrow\left(\mathfrak{L}^{2}, \pi_{v P v^{-1}}^{\prime}\right)$, and it follows from (171) that for every $f \in L^{2}(G / H)$ we have:

$$
\mathfrak{F}_{s P s^{-1}}^{\prime} f=m(v)\left[\mathfrak{F}_{P} f\right] \text {. }
$$

One readily verifies that the map $\mathcal{I}\left(\widehat{\mathbf{M}}_{H}, v \widehat{\mathbf{M}}_{H}\right) \circ m(v): \mathfrak{L}^{2} \rightarrow \mathfrak{L}^{2}$ only depends on the image $s$ of $v$ in $W$. Therefore we denote it by $\mathbf{m}(s)$. We now have the following.

Lemma 19.5 Let $s \in W$. Then the map $\mathbf{m}(s)$ is an equivariant unitary isomorphism from $\left(\mathfrak{L}^{2}, \pi_{P}\right)$ onto $\left(\mathfrak{L}^{2}, \pi_{s P s^{-1}}\right)$. Moreover, $\mathfrak{F}_{s P^{-1}}=\mathbf{m}(s) \circ \mathfrak{F}_{P}$.

Corollary 19.6 For every $s \in W$ we have, $(s)=\mathfrak{A}\left(P: s P s^{-1}\right) \circ \mathbf{m}(s)$.

Proof. Fix $s \in W$ and write $a$ for the operator on the right-hand side of the above equation. From the definitions given above we infer that there exists a measurable function $\lambda \mapsto b(\lambda)$ with values in the space of unitary maps $\mathfrak{H} \rightarrow \mathfrak{H}$ such that for $\varphi \in \mathfrak{L}^{2}$ we have $[a \varphi](\lambda)=b\left(s^{-1} \lambda\right) \varphi\left(s^{-1} \lambda\right)$. Combining Lemmas 19.3 and 19.5 we see that $\hat{f}=a \hat{f}$ for every $f \in C_{c}^{\infty}(G / H)$. Hence

$$
\hat{f}(s \lambda)=b(\lambda) \hat{f}(\lambda) .
$$

By the uniqueness part of Proposition 18.6 this implies that $b(\lambda)=\mathfrak{C}_{s}(\lambda)$. Now use definition (158) of , $(s)$ to conclude that $a=,(s)$.

Remark 19.7 Corollary 19.6 essentially expresses the relation between $\hat{f}(s \lambda)$ and $\hat{f}(\lambda)$ by means of standard intertwining operators. In the Riemannian case a similar relation is given in [31], Ch. VI, Cor. 3.9.

\section{Properties of the pre Paley-Wiener spaces}

In this section we collect some properties of the pre Paley-Wiener spaces introduced in Definition 8.9. These properties will be needed in the next section. Let $P \in \mathcal{P}_{\sigma}^{\min }$ be fixed, let $\left(\tau, V_{\tau}\right)$ be a finite dimensional unitary representation of $K$, and let $\mathcal{F}=\mathcal{F}_{P}$ be the associated $\tau$-spherical Fourier transform.

Let $\pi \in \Pi_{\Sigma}\left(\mathfrak{a}_{\mathrm{q}}\right)$ be as in $(64)$ and let $S$ be a compact $W_{K \cap H}$-invariant subset of $\mathfrak{a}_{\mathrm{q}}$. Moreover, let $\epsilon>0$ be as in Lemma 8.1. If $f \in C_{S}^{\infty}(G / H: \tau)$, then by Theorem 8.11 the function $\mathcal{F} f$ belongs to the pre Paley-Wiener space $\mathcal{M}(S, \pi)$. Moreover, it has the property that $\pi \mathcal{F} f$ is holomorphic on the open neighbourhood $\mathfrak{a}_{\mathrm{q}}^{*}(P, \epsilon)$ of $\overline{\mathfrak{a}}_{\mathrm{q}}^{*}(P, 0)$. We will show that the positive constant $\epsilon$ can be fixed so that any function in $\mathcal{M}(S, \pi)$ has this property. More generally, we establish a similar result for the pre Paley-Wiener space $\mathcal{M}(S, p)$, under the assumption that $p \in \Pi_{\Sigma}\left(\mathfrak{a}_{\mathrm{q}}\right)$. 
For convenience we introduce, for $p \in \Pi\left(\mathfrak{a}_{\mathrm{q}}\right)$, the space $\mathcal{M}(p)$ of meromorphic functions $\varphi: \mathfrak{a}_{\mathrm{qc}}^{*} \rightarrow{ }^{\circ} \mathcal{C}(\tau)$ which satisfy conditions (a) and (b) of Definition 8.9. We equip this space with the locally convex topology determined by the set of seminorms

$$
\nu_{V}: \varphi \mapsto \sup _{\lambda \in V}\|p \varphi(\lambda)\|
$$

with $V$ a bounded subset of $\overline{\mathfrak{a}}_{\mathrm{q}}^{*}(P, 0)$. Then $\mathcal{M}(S, p)$ is a linear subspace of $\mathcal{M}(p)$; moreover the inclusion map is continuous.

If $U$ is a complex manifold, then by $\mathcal{O}(U)$ we denote the space of holomorphic functions $U \rightarrow \mathbb{C}$, equipped with the usual Fréchet topology.

Proposition 20.1 Let $p \in \Pi_{\Sigma}\left(\mathfrak{a}_{\mathrm{q}}\right)$. Then we have the following.

(a) For every $r>0$ there exists a polynomial function $q_{r} \in \Pi_{\Sigma}\left(\mathfrak{a}_{\mathrm{q}}\right)$ such that the following holds. For every $\varphi \in \mathcal{M}(p)$ the function $q_{r} \varphi$ is holomorphic on $\mathfrak{a}_{\mathrm{q}}^{*}(P, r)$; moreover, the map $\left.\varphi \mapsto q_{r} \varphi\right|_{\mathfrak{a}_{\mathrm{q}}^{*}(P, r)}$ is continuous from $\mathcal{M}(p)$ to $\mathcal{O}\left(\mathfrak{a}_{\mathrm{q}}^{*}(P, r)\right) \otimes{ }^{\circ} \mathcal{C}(\tau)$.

(b) There exists a constant $\epsilon>0$ such that for every $\varphi \in \mathcal{M}(p)$ the function $p \varphi$ is regular on $\mathfrak{a}_{\mathrm{q}}^{*}(P, \epsilon)$. Moreover, the map $\left.\varphi \mapsto p \varphi\right|_{\mathfrak{a}_{\mathrm{q}}^{*}(P, \epsilon)}$ is continuous from $\mathcal{M}(p)$ to $\mathcal{O}\left(\mathfrak{a}_{\mathrm{q}}^{*}(P, \epsilon)\right) \otimes{ }^{\circ} \mathcal{C}(\tau)$.

(c) The space $\mathcal{M}(p)$ is Fréchet.

Before giving the proof of this result, we derive the following corollary from it.

Corollary 20.2 Let $p \in \Pi_{\Sigma}\left(\mathfrak{a}_{\mathrm{q}}\right)$. Then we have the following.

(a) There exists a constant $\epsilon>0$ such that for any $W_{K \cap H}$-invariant compact set $S \subset \mathfrak{a}_{\mathrm{q}}^{*}$ the following holds. For every $\varphi \in \mathcal{M}(S, p)$ the function $p \varphi$ is regular on $\mathfrak{a}_{\mathrm{q}}^{*}(P, \epsilon)$; moreover, the map $\left.\varphi \mapsto p \varphi\right|_{\mathfrak{a}_{\mathrm{q}}^{*}(P, \epsilon)}$ is continuous from $\mathcal{M}(S, p)$ to $\mathcal{O}\left(\mathfrak{a}_{\mathrm{q}}^{*}(P, \epsilon)\right) \otimes{ }^{\circ} \mathcal{C}(\tau)$.

(b) For every $W_{K \cap H}$-invariant compact set $S \subset \mathfrak{a}_{\mathrm{q}}$ the pre Paley-Wiener space $\mathcal{M}(S, p)$ is Fréchet.

Proof. If $S \subset \mathfrak{a}_{\mathrm{q}}$ is any $W_{K \cap H}$-invariant compact subset, then $\mathcal{M}(S, p) \subset \mathcal{M}(p)$, the inclusion map being continuous. Hence (a) follows from Proposition 20.1 (b) with the same positive constant $\epsilon$.

To see that (b) holds, let $S \subset \mathfrak{a}_{\mathrm{q}}^{*}$ be compact and $W_{K \cap H}$-invariant. Then $\mathcal{M}(S, p)$ has a countable system of neighborhoods of the origin, hence is metrizable. Let $\left(\varphi_{n}\right)$ be a Cauchy sequence in $\mathcal{M}(S, p)$; then we must show it converges in $\mathcal{M}(S, p)$. By continuity of the inclusion map the sequence $\left(\varphi_{n}\right)$ is Cauchy in $\mathcal{M}(p)$, hence converges in $\mathcal{M}(p)$; let $\varphi$ be its limit. Then $p \varphi_{n} \rightarrow p \varphi$ pointwise on $\overline{\mathfrak{a}}_{\mathrm{q}}^{*}(P, 0)$. Hence if $\nu$ is any of the seminorms of Definition $8.9(\mathrm{c})$, then $\nu\left(\varphi_{n}-\varphi\right) \leq \sup _{m \geq n} \nu\left(\varphi_{n}-\varphi_{m}\right)$. Since $\left(\varphi_{n}\right)$ is a Cauchy sequence in $\mathcal{M}(S, p)$ it now follows that $\varphi \in \mathcal{M}(S, p)$ and that $\left(\varphi_{n}\right)$ converges to $\varphi$ in $\mathcal{M}(S, p)$ as well. Thus we have established property (b) 
For the proof of Proposition 20.1 we need information on the poles of the $C$-functions. We start with some results on standard intertwining operators. For $P, Q \in \mathcal{P}_{\sigma}^{\min },[\xi] \in$ $\widehat{M}_{\mathrm{fu}}, \lambda \in \mathfrak{a}_{\mathrm{q} \mathbf{c}}^{*}$, let $A(Q: P: \xi: \lambda)$ denote the standard intertwining operator from $\pi_{P, \xi, \lambda}$ to $\pi_{Q, \xi, \lambda}$, defined as in [6]. We recall from the text preceding (24) that $A(Q: P: \xi: \lambda)$ may be viewed as a continuous linear endomorphism of $C^{-\infty}(K: \xi)$, depending meromorphically on $\lambda$.

From [6], Props. 4.7 and 4.8, we recall that there exists a unique non-trivial meromorphic function $\eta(Q: P: \xi): \mathfrak{a}_{\mathbf{q}}^{*} \rightarrow \mathbb{C}$ such that

$$
A(P: Q: \xi: \lambda) \circ A(Q: P: \xi: \lambda)=\eta(Q: P: \xi: \lambda) I,
$$

for generic $\lambda \in \mathfrak{a}_{\mathrm{qc}}^{*}$.

We shall describe the poles of the intertwining operators, viewed as meromorphic functions of $\lambda$, in some detail, first on the $K$-finite level. If $\vartheta \subset \widehat{K}$ is a finite subset, then we denote by $C(K: \xi)_{\vartheta}$ the finite dimensional subspace of right $K$-finite functions in $C^{-\infty}(K: \xi)$, all of whose right $K$-types belong to $\vartheta$. Moreover, we denote the restriction of the standard intertwining operator to this space by $A(Q: P: \xi: \lambda)_{\vartheta}$.

Lemma 20.3 Let $P, Q \in \mathcal{P}_{\sigma}^{\min }$ and let $U \subset \mathfrak{a}_{\mathrm{qc}}^{*}$ be an open subset such that for every $\alpha \in \Sigma(Q, P):=\Sigma(Q) \cap \Sigma(\bar{P})$ the function $\lambda \mapsto|\operatorname{Re}\langle\lambda, \alpha\rangle|$ is bounded on $U$. Then there exists a polynomial $q \in \Pi_{\Sigma(Q, P)}\left(\mathfrak{a}_{q}\right)$ such that for every finite subset $\vartheta \subset \widehat{K}$ we have the following:

(a) The $\operatorname{End}\left(C(K: \xi)_{\vartheta}\right)$-valued meromorphic functions $\lambda \mapsto q(\lambda) A(Q: P: \xi: \lambda)_{\vartheta}$ and $\lambda \mapsto q(\lambda) A(Q: P: \xi: \lambda)_{\vartheta}^{-1}$ are regular on $U$.

(b) The meromorphic functions $\lambda \mapsto q(\lambda) \eta(Q: P: \xi: \lambda)$ and $\lambda \mapsto q(\lambda) \eta(Q: P: \xi: \lambda)^{-1}$ are regular on $U$.

Proof. The existence of a $q$ such that the function $\lambda \mapsto q(\lambda) A(Q: P: \xi: \lambda)_{\vartheta}$ is regular on $U$ for any finite set $\vartheta \subset \widehat{K}$ is a straightforward consequence of [33], Thm. 6.6. This proves the first part of assertion (a).

The existence of a $q$ such that (b) holds follows if we restrict (173) to an arbitrary fixed $K$-isotypical component of $C^{-\infty}(K: \xi)$ and apply [7], Lemma 16.6, to the resulting restrictions of the intertwining operators.

Finally, the existence of a $q$ for which the remaining part of (a) holds follows from the first part of (a), combined with (173) and (b).

For $r \in \mathbb{N}$, let $C^{-r}(K: \xi)$ denote the space of generalized functions in $C^{-\infty}(K: \xi)$ of order at most $r$. By compactness of $K$ this space carries a Banach topology (see [6], $\$ 4$, where the notation $\mathcal{D}_{r}^{\prime}$ is used instead of $\left.C^{-r}\right)$. In the following we shall use the notation $B\left(C^{-r}, C^{-r^{\prime}}\right)$ for the Banach space of bounded linear operators $C^{-r}(K: \xi) \rightarrow C^{-r^{\prime}}(K: \xi)$, equipped with the operator norm $\left(r, r^{\prime} \in \mathbb{N}\right)$. 
Corollary 20.4 Let the assumptions of Lemma 20.3 be fulfilled and let $q \in \Pi_{\Sigma\left(P_{2}, P_{1}\right)}$ be such that for any finite subset $\vartheta \subset \widehat{K}$ assertion (a) holds. Then for every relatively compact open subset $\Omega \subset U$ there exists a constant $s \in \mathbb{N}$, such that for every $r \in \mathbb{N}$ we have the following:

(a) the mapping $(\lambda, f) \mapsto q(\lambda) A(Q: P: \xi: \lambda) f$ maps $\Omega \times C^{-r}(K: \xi)$ continuously into $C^{-r-s}(K: \xi)$;

(b) the induced function $\lambda \mapsto q(\lambda) A(Q: P: \xi: \lambda) \mid C^{-r}(K: \xi)$ from $\Omega$ to the Banach space $B\left(C^{-r}, C^{-r-s}\right)$ is holomorphic.

Proof. By density of the $K$-finite functions the result follows if we combine Lemma 20.3 (a) with Prop. 4.11 of [6].

Recall the introduction of the meromorphic $\operatorname{End}(V(\xi))$-valued map $B(Q: P: \xi)$ on $\mathfrak{a}_{\mathrm{q}}^{*}$ by the property (167).

Lemma 20.5 Let $P, Q \in \mathcal{P}_{\sigma}^{\min }$. Moreover, let $U \subset \mathfrak{a}_{\mathrm{q}}^{*}$ be an open subset such that for every $\alpha \in \Sigma(Q, P):=\Sigma(Q) \cap \Sigma(\bar{P})$ the function $\lambda \mapsto|\langle\operatorname{Re} \lambda, \alpha\rangle|$ is bounded on $U$. Then there exists a polynomial function $q \in \Pi_{\Sigma(Q, P)}\left(\mathfrak{a}_{\mathrm{q}}\right)$ such that the End $(V(\xi))$-valued meromorphic functions

$$
\lambda \mapsto q(\lambda) B(Q: P: \xi: \lambda) \quad \text { and } \quad \lambda \mapsto q(\lambda) B(Q: P: \xi: \lambda)^{-1}
$$

are regular on $U$.

Proof. By the product decomposition of [6], Prop. 7.1, we see that it suffices to prove this result if $P, Q$ are $\sigma$-adjacent, i.e. $P \neq Q$ and all roots in $\Sigma(Q, P)$ are proportional. Assume this to be the case and let $\alpha$ be the reduced root in $\Sigma(Q, P)$. Then $\alpha$ is a simple root for the positive system $\Sigma(\bar{P})$, and $Q=P^{s_{\alpha}}$, the conjugate of $P$ under the reflection $s_{\alpha} \in W$ in the root hyperplane $\alpha$. Moreover, $\mathbb{C}^{*} \Pi_{\Sigma(Q, P)}\left(\mathfrak{a}_{\mathrm{q}}\right)=\mathbb{C}^{*} \Pi_{\{\alpha\}}\left(\mathfrak{a}_{\mathrm{q}}\right)$ and the condition on $U$ is equivalent to: $\lambda \mapsto|\operatorname{Re}\langle\lambda, \alpha\rangle|$ is bounded on $U$.

We recall from Section 2 that the set $\mathcal{W}$ is in bijective correspondence with $W / W_{K \cap H}$. Accordingly we transfer the action by left multiplication of $W$ on $W / W_{K \cap H}$ to an action on $\mathcal{W}$, denoted $(s, v) \mapsto s \cdot v$. If $w \in \mathcal{W}$, we write $V(\xi, w)$ for the summand $\mathcal{H}_{\xi}^{w H_{\mathrm{M}} w^{-1}}$ in the direct sum decomposition (9).

Let $w \in \mathcal{W}$. Then the endomorphism $B(Q: P: \xi: \lambda)$ leaves the subspace $V(\xi, w)+$ $V\left(\xi, s_{\alpha} \cdot w\right)$ of $V(\xi)$ invariant; see [6], Lemma 7.2. We denote its restriction to that subspace by $B_{w}(Q: P: \xi: \lambda)$. It now suffices to establish the existence of a $q \in \Pi_{\{\alpha\}}\left(\mathfrak{a}_{q}\right)$ such that the endomorphisms $q(\lambda) B_{w}(Q: P: \xi: \lambda)^{ \pm 1}$ depend holomorphically on $\lambda \in U$.

By [6], Lemma 6.10 and eqn. (7.1), there exists a unitary bijection $L=L\left(w^{-1} \xi, w\right)$ from $V\left(w^{-1} \xi\right)$ onto $V(\xi)$, mapping $V\left(w^{-1} \xi, 1\right)+V\left(w^{-1} \xi, s_{w^{-1} \alpha} \cdot 1\right)$ onto $V(\xi, w)+V\left(\xi, s_{\alpha}\right.$. $w)$, such that

$$
B_{w}(Q: P: \xi: \lambda)=L \circ B_{1}\left(w^{-1} Q w: w^{-1} P w: w^{-1} \xi: w^{-1} \lambda\right) \circ L^{-1} .
$$


Notice that $w^{-1} \alpha$ is a simple root in $\Sigma\left(w^{-1} P w\right)$, that $w^{-1} Q w=\left(w^{-1} P w\right)^{s} w^{-1} \alpha$, and that $w^{-1} \Pi_{\{\alpha\}}\left(\mathfrak{a}_{\mathbf{q}}\right)=\Pi_{\left\{w^{-1} \alpha\right\}}\left(\mathfrak{a}_{\mathrm{q}}\right)$. We now see from (175) that it suffices to prove the existence of a $q \in \Pi_{\{\alpha\}}\left(\mathfrak{a}_{\mathrm{q}}\right)$ such that $q(\lambda) B_{1}\left(P^{s_{\alpha}}: P: \xi: \lambda\right)^{ \pm 1}$ depend holomorphically on $\lambda \in U$, for arbitrary $\xi \in \widehat{M}_{H}, P \in \mathcal{P}_{\sigma}^{\text {min }}, \alpha$ any simple root in $\Sigma(\bar{P})$, and finally $U$ any open subset of $\mathfrak{a}_{\mathrm{q} c}^{*}$ with $\lambda \mapsto|\operatorname{Re}\langle\lambda, \alpha\rangle|$ bounded on $U$. Using the split rank one reduction of [6], Lemma 7.4, (where $B_{1}$ is denoted $B_{s_{\alpha}}$ ) we now see that it suffices to prove the lemma if $\operatorname{dim} \mathfrak{a}_{\mathrm{q}}=1$, and if $Q=\bar{P}$. In the rest of the proof we assume this to be the case. Let $\alpha$ be the reduced root in $\Sigma(\bar{P})$. The condition on $U$ means that $U \subset \mathfrak{a}_{\mathrm{q}}^{*}(R)$ for some $R>0$.

Following [6], $\S 5$, we define, for any $Q \in \mathcal{P}_{\sigma}^{\min }$, the map ev: $C^{-\infty}(Q: \xi: \lambda)^{H} \rightarrow V(\xi)$ by $\operatorname{ev}(f)_{w}=f(w), w \in \mathcal{W}$. Then ev $\circ(Q: \xi: \lambda)=I_{V(\xi)}$, for generic $\lambda \in \mathfrak{a}_{\mathrm{qc}}^{*}$. Using (167) we now obtain the following identity of endomorphisms of $V(\xi)$ for generic $\lambda \in \mathfrak{a}_{\mathrm{q}}^{*}$ :

$$
B(\bar{P}: P: \xi: \lambda)=\operatorname{ev} \circ A(\bar{P}: P: \xi: \lambda) \circ j(P: \xi: \lambda) .
$$

By [7], Thm. 9.1, there exist a polynomial $q_{1} \in \Pi_{\Sigma}\left(\mathfrak{a}_{\mathrm{q}}\right)$ and a constant $r \in \mathbb{N}$ such that $q_{1}(\lambda) j(P: \xi: \lambda) \eta$ depends holomorphically on $\lambda \in \mathfrak{a}_{\mathrm{q}}^{*}(R)$ as an element of $C^{-r}(K: \xi)$, for every $\eta \in V(\xi)$. Moreover, by Corollary 20.4 there exists a polynomial $q_{2} \in \Pi_{\Sigma}\left(\mathfrak{a}_{\mathrm{q}}\right)$ such that $q_{2}(\lambda) A(\bar{P}: P: \xi: \lambda)$ depends holomorphically on $\lambda \in \mathfrak{a}_{\mathrm{q}}^{*}(R)$ as an element of the space $B\left(C^{-r}, C^{-r-s}\right)$. Write $q=q_{1} q_{2}$. Then we see that $f_{\eta}(\lambda):=q(\lambda) A(\bar{P}: P: \xi: \lambda) j(P: \xi: \lambda) \eta$ depends holomorphically on $\lambda \in \mathfrak{a}_{\mathrm{q}}^{*}(R)$, as an element of $C^{-r-s}(K: \xi)$. Moreover, $f_{\eta}(\lambda)$ is $\pi_{\xi, \lambda}(H)$-invariant, hence $(\lambda, h) \mapsto \pi_{\xi, \lambda}(h) f_{\eta}(\lambda)$ is a smooth map from $\mathfrak{a}_{\mathrm{q}}^{*}(R) \times H$ to $C^{-r-s}(K: \xi)$, which is holomorphic in the first variable. It now follows by application of $[6]$, Lemma 4.13, that $\operatorname{ev}\left(f_{\eta}(\lambda)\right) \in V(\xi)$ depends holomorphically on $\lambda \in \mathfrak{a}_{\mathrm{q}}^{*}(R)$, for every $\eta \in V(\xi)$. We conclude that $q(\lambda)$ times the endomorphism on the right-hand side of (176) depends holomorphically on $\lambda \in \mathfrak{a}_{\mathrm{q}}^{*}(R)$, and the assertion about $B$ follows. For the assertion about $B^{-1}$ we observe that it follows from [6], Prop. 6.2 (i), that

$$
B(\bar{P}: P: \xi: \lambda)^{-1}=\eta(P: \bar{P}: \xi: \lambda)^{-1} B(P: \bar{P}: \xi: \lambda) .
$$

Now combine Lemma 20.3 (b) with the result obtained for $B$ to complete the proof.

Lemma 20.6 Let $P, Q \in \mathcal{P}_{\sigma}^{\min }, s \in W$. Moreover, let $U$ be a an open subset of $\mathfrak{a}_{\mathrm{qc}}^{*}$ such that for each $\alpha \in \Sigma(\bar{P}) \cap s^{-1} \Sigma(Q)$ the function $\lambda \mapsto|\operatorname{Re}\langle\lambda, \alpha\rangle|$ is bounded on $U$. Then there exists a polynomial function $q \in \Pi_{\Sigma(\bar{P}) \cap s^{-1} \Sigma(Q)}\left(\mathfrak{a}_{\mathrm{q}}\right)$ such that the endomorphism

$$
q(\lambda) C_{Q \mid P}^{\circ}(s: \lambda)
$$

of ${ }^{\circ} \mathrm{C}(\tau)$ depends holomorphically on $\lambda \in U$.

Proof. For $s \in W$, let $\mathcal{L}(s)$ be the unitary endomorphism of ${ }^{\circ} \mathcal{C}(\tau)$ defined in $[13], \S 7$. Then by loc.cit., Lemma 7, we have the transformation rule

$$
C_{Q \mid P}^{\circ}(s: \lambda)=\mathcal{L}(s) \circ C_{s^{-1} Q s \mid P}^{\circ}(1: \lambda),
$$

as a meromorphic identity in $\lambda \in \mathfrak{a}_{\mathrm{q} c}^{*}$. From this we see that it suffices to prove the assertion for $s=1$ and arbitrary $P, Q \in \mathcal{P}_{\sigma}^{\min }$. 
By $(27)$ the space ${ }^{\circ} \mathcal{C}(\tau)$ admits an orthogonal direct sum decomposition into subspaces ${ }^{\circ} \mathcal{C}_{\xi}(\tau)$, where $[\xi]$ ranges over finitely many classes in $\widehat{M}_{H}$. Moreover, the endomorphism $C_{Q \mid P}^{\circ}(1: \lambda)$ leaves every subspace of this decomposition invariant. Let $[\xi] \in \widehat{M}_{H}$ occur in the decomposition. Then it suffices to prove the existence of a $q \in \Pi_{\Sigma(\bar{P}) \cap \Sigma(Q)}\left(\mathfrak{a}_{\mathrm{q}}\right)$ such that the restriction to ${ }^{\circ} \mathcal{C}_{\xi}(\tau)$ of the endomorphism (177) with $s=1$ depends holomorphically on $\lambda \in U$. This follows immediately from [13], Lemma 3 and eqn. (57), combined with Lemmas 20.3 and 20.5 above.

If $U \subset \mathfrak{a}_{\mathrm{qc}}^{*}$ is an open subset, and $q$ a polynomial function in $\Pi\left(\mathfrak{a}_{\mathrm{q}}\right)$, we denote the map $\mathcal{O}(U) \rightarrow \mathcal{O}(U), \varphi \mapsto q \varphi$ by $m_{q}$. The image of $m_{q}$ is denoted by $\mathcal{O}(U, q)$ and equipped with the topology inherited from the ambient space $\mathcal{O}(U)$.

Lemma 20.7 Let $U \subset \mathfrak{a}_{\mathrm{qc}}^{*}$ be an open subset, and let $q \in \Pi\left(\mathfrak{a}_{\mathrm{q}}\right)$. Then the map $m_{q}$ is a topological linear isomorphism from $\mathcal{O}(U)$ onto $\mathcal{O}(U, q)$.

Proof. Since $\mathcal{O}(U)$ has no zero divisors, it is clear that $m_{q}$ is a linear isomorphism from $\mathcal{O}(U)$ onto $\mathcal{O}(U, q)$. Moreover, it is obvious that $m_{q}$ is continuous. For the continuity of its inverse one needs to estimate $\varphi$ in terms of $q \varphi$, for $\varphi \in \mathcal{O}(U)$. The required estimates can be obtained by a repeated application of Cauchy's integral formula in the same fashion as in the proof of Lemma 6.1 in [7].

Proof of Proposition 20.1. We first prove (a). Let $r>0$. For $s \in W$, put

$$
U_{s}=s^{-1} \mathfrak{a}_{\mathrm{q}}^{*}(P, r) \cap \overline{\mathfrak{a}}_{\mathrm{q}}^{*}(P, 0) .
$$

Then $\mathfrak{a}_{\mathrm{q}}^{*}(P, r)$ is the union of the sets $s U_{s}, s \in W$.

Fix $s \in W$ for the moment, and let $\alpha \in s^{-1} \Sigma(P) \cap \Sigma(\bar{P})$. Then for $\lambda \in U_{s}$ we have $\operatorname{Re}\langle\lambda, \alpha\rangle \geq 0$. For such $\lambda$ we also have $\operatorname{Re}\langle\lambda, \alpha\rangle=\operatorname{Re}\langle s \lambda, s \alpha\rangle<r$. Hence $\lambda \mapsto$ $|\operatorname{Re}\langle\lambda, \alpha\rangle|$ is bounded on $U_{s}$. By Lemma 20.6 it now follows that there exists a polynomial function $q_{s} \in \Pi_{\Sigma}\left(\mathfrak{a}_{\mathrm{q}}\right)$ such that the function $\lambda \mapsto q_{s}(\lambda) C_{P \mid P}^{\circ}(s: \lambda)$ is regular on an open neighborhood of $U_{s}$, hence locally bounded on $U_{s}$. Let now $\varphi \in \mathcal{M}(p)$. Since $\varphi$ satisfies conditions (a) and (b) of Definition 8.9, it follows that the function

$$
\lambda \mapsto q_{s}(\lambda) p(\lambda) \varphi(s \lambda)
$$

is locally bounded on $U_{s}$. We now define the polynomial $q \in \Pi_{\Sigma}\left(\mathfrak{a}_{\mathrm{q}}\right)$ by

$$
q(\lambda)=\prod_{s \in W} q_{s}\left(s^{-1} \lambda\right) p\left(s^{-1} \lambda\right) .
$$

Then the function $q \varphi$ is locally bounded on $s U_{s}$ for every $s \in W$. These sets are closed in the set $\mathfrak{a}_{\mathrm{q}}^{*}(P, r)$ and cover it. Therefore the function $q \varphi$ is locally bounded, hence holomorphic, on $\mathfrak{a}_{\mathrm{q}}^{*}(P, r)$. Moreover, from the above reasoning we also see that the map $\left.\varphi \mapsto q \varphi\right|_{\mathfrak{a}_{\mathrm{q}}^{*}(P, r)}$ is continuous.

We now turn to the proof of $(b)$. Let $q \in \Pi_{\Sigma}\left(\mathfrak{a}_{q}\right)$ be a polynomial as in (a) for $r=1$. Let $\varphi \in \mathcal{M}(p)$. Then $q p \varphi$ is regular on $\mathfrak{a}_{\mathrm{q}}^{*}(P, 1)$, and $p \varphi$ is regular on $\overline{\mathfrak{a}}_{\mathrm{q}}^{*}(P, 0)$, hence on the 
smaller set $i \mathfrak{a}_{\mathbf{q}}^{*} \cup \mathfrak{a}_{\mathrm{q}}^{*}(P, 0)$. By Lemma 8.2, applied with $\eta_{1}=1$ and with $q$ in place of $p$, it follows that there exists a constant $\epsilon \in] 0,1[$, depending only on $q$, such that $p \varphi$ is regular on $\mathfrak{a}_{\mathrm{q}}^{*}(P, \epsilon)$. This establishes the first assertion of $(\mathrm{b})$. For the second assertion, we note that by (a) the map $R:\left.\varphi \mapsto q p \varphi\right|_{\mathfrak{a}_{\mathrm{q}}^{*}(P, \epsilon)}$ is continuous from $\mathcal{M}(p)$ to $\mathcal{O}\left(\mathfrak{a}_{\mathrm{q}}^{*}(P, \epsilon)\right) \otimes{ }^{\circ} \mathcal{C}(\tau)$. We have just seen that its image is contained in $\mathcal{O}\left(\mathfrak{a}_{\mathrm{q}}^{*}(P, \epsilon), q\right)$. Thus we may apply Lemma 20.7 and compose the map $R$ with $m_{q}^{-1} \otimes I^{\circ} \mathcal{C}(\tau)$ to establish the validity of (b).

Finally we prove $(c)$. Since $\mathcal{M}(p)$ has a countable system of neighborhoods of the origin it is metrizable, and it suffices to prove completeness.

Let $\left(\varphi_{n}\right)_{n \in \mathbb{N}}$ be a Cauchy sequence in $\mathcal{M}(p)$. For each $r>0$ we write $\Omega_{r}=\mathfrak{a}_{\mathrm{q}}^{*}(P, r)$, and we select $q_{r} \in \Pi_{\Sigma}\left(\mathfrak{a}_{\mathrm{q}}\right)$ as in assertion (a). Then the sequence $\left(q_{r} \varphi_{n} \mid \Omega_{r}\right)$ is a Cauchy sequence in the Fréchet space $\mathcal{O}\left(\Omega_{r}\right) \otimes{ }^{\circ} \mathcal{C}(\tau)$, hence converges to a limit $\psi_{r} \in \mathcal{O}\left(\Omega_{r}\right) \otimes{ }^{\circ} \mathcal{C}(\tau)$. We define the meromorphic function $\phi_{r}$ on $\Omega_{r}$ by $\phi_{r}=q_{r}^{-1} \psi_{r}$. Let now $r, r^{\prime}>0$. Then $q_{r} q_{r^{\prime}} \varphi_{n} \mid \Omega_{r^{\prime}} \rightarrow q_{r} \psi_{r^{\prime}}=q_{r} q_{r^{\prime}} \phi_{r^{\prime}}$, locally uniformly on $\Omega_{r^{\prime}}$. Similarly, we see that $q_{r} q_{r^{\prime}} p_{0} \varphi_{n} \mid \Omega_{r} \rightarrow q_{r} q_{r^{\prime}} \phi_{r}$, locally uniformly on $\Omega_{r}$. It follows that $\phi_{r}=\phi_{r^{\prime}}$ on $\Omega_{r} \cap \Omega_{r^{\prime}}=$ $\Omega_{\min \left(r, r^{\prime}\right)}$. Now the union of the sets $\Omega_{r}, r>0$, equals $\mathfrak{a}_{\mathrm{qc}}^{*}$. Hence there exists a meromorphic function $\varphi: \mathfrak{a}_{\mathrm{q}}^{*} \mathbf{c}{ }^{\circ} \mathcal{C}(\tau)$ such that $\varphi=\phi_{r}$ on $\Omega_{r}$, for every $r>0$. We will show that $\varphi$ belongs to $\mathcal{M}(p)$ and is the limit of the sequence $\left(\varphi_{n}\right)$.

By the definition of $\varphi$ we have that $q_{r} \varphi_{n}$ converges to $q_{r} \varphi$ in $\mathcal{O}\left(\Omega_{r}\right) \otimes{ }^{\circ} \mathcal{C}(\tau)$ for all $r>0$. In particular it follows that $\varphi_{n}(\lambda) \rightarrow \varphi(\lambda)$ for generic $\lambda \in \mathfrak{a}_{\mathrm{qc}}^{*}$. From this it follows that $\varphi$ fulfills condition (a) of Definition 8.9.

Let $\epsilon>0$ be the constant of the already established property (b). Then $\left(p \varphi_{n} \mid \Omega_{\epsilon}\right)$ is a Cauchy sequence in $\mathcal{O}\left(\Omega_{\epsilon}\right) \otimes{ }^{\circ} \mathcal{C}(\tau)$. Let $f$ be its limit. By a straightforward passage to limits we have $q_{\epsilon} f=p \psi_{\epsilon}=p q_{\epsilon} \varphi$. Hence $f=p \varphi$, and we conclude that $p \varphi$ is holomorphic on $\Omega_{\epsilon}$, and that the sequence $\left(p \varphi_{n}\right)$ converges locally uniformly to $p \varphi$ on $\Omega_{\epsilon}$. In particular this means that $\varphi$ fulfills condition (b) of Definition 8.9, hence belongs to $\mathcal{M}(p)$, and that $\varphi_{n} \rightarrow \varphi$ with respect to the seminorms (172). This completes the proof of property (c).

\section{Paley-Wiener theorems}

In this section we assume that $P \in \mathcal{P}_{\sigma}^{\min }$ and that $\tau$ is a finite dimensional unitary representation of $K$. Our objective is to investigate the image of the space $C_{c}^{\infty}(G / H: \tau)$ under the $\tau$-spherical Fourier transform $\mathcal{F}=\mathcal{F}_{P}$.

If $S \subset \mathfrak{a}_{\mathrm{q}}$ is a closed $W_{K \cap H}$-invariant subset, then we define the closed subset $X_{S}$ of $G / H$ by (66). Recall also the definition (73) of the supporting function $h_{T}: \mathfrak{a}_{\mathrm{qc}}^{*} \rightarrow$ $[-\infty, \infty]$, when $T$ is a closed set of $\mathfrak{a}_{\mathrm{q}}$. Define the polynomial $\pi \in \Pi_{\Sigma}\left(\mathfrak{a}_{\mathrm{q}}\right)$ as in $(64)$. Then we have the following Paley-Wiener type characterization of the support of a compactly supported function in terms of its Fourier transform.

Theorem 21.1 Let $f \in C_{c}^{\infty}(G / H: \tau)$, and let $S$ be any $W_{K \cap H}$-invariant compact convex subset of $\mathfrak{a}_{\mathrm{q}}$. Then supp $f \subset X_{S}$ if and only if for every $n \in \mathbb{N}$ there exists a constant 
$C>0$ such that for every $w \in \mathcal{W}$ and $\lambda \in \overline{\mathfrak{a}}_{\mathrm{q}}^{*}(P, 0)$ we have

$$
\left\|\pi(\lambda) \operatorname{pr}_{w} \mathcal{F} f(\lambda)\right\| \leq C(1+|\lambda|)^{-n} e^{h_{w S}(-\lambda)} .
$$

Proof. The implication 'only if' is immediate from Theorem 8.11 and Definition 8.9 (c). Since $f$ is compactly supported, there exists a $W_{K \cap H}$-invariant compact subset $T \subset \mathfrak{a}_{\mathrm{q}}$ such that supp $f \subset X_{T}$. By Theorem 8.11 we have that $\mathcal{F} f \in \mathcal{M}(T, \pi)$. Hence $\mathcal{F} f$ satisfies conditions (a) - (b) of Definition 8.9. If we combine these with the estimate (178), we see that in fact $\mathcal{F} f \in \mathcal{M}(S, \pi)$. Fix $D \in \mathbb{D}_{\pi}^{\prime}$ (cf. Lemma 15.3). Then from Corollary 10.4 (and Remark 10.5) we obtain that

$$
\operatorname{supp} D \mathcal{J F} f \subset X_{S}
$$

By Theorem 14.1 this implies that

$$
\operatorname{supp} D f \subset X_{S}
$$

In view of Proposition 15.2 we finally conclude that supp $f \subset X_{S}$.

Definition 21.2 The pre Paley-Wiener space $\mathcal{M}(G / H: \tau)$ is defined to be the space of meromorphic functions $\varphi: \mathfrak{a}_{\mathrm{qc}}^{*} \rightarrow{ }^{\circ} \mathrm{C}(\tau)$ having the following properties:

(a) $\varphi(s \lambda)=C_{P \mid P}^{\circ}(s: \lambda) \varphi(\lambda)$ for all $s \in W$, and generic $\lambda \in \mathfrak{a}_{\mathrm{qc}}^{*}$;

(b) The function $\pi \varphi$ is holomorphic on an open neighborhood of $\overline{\mathfrak{a}}_{\mathrm{q}}^{*}(P, 0)$;

(c) There exists a constant $R>0$ and for every $n \in \mathbb{N}$ a constant $C>0$, such that for all $\lambda \in \overline{\mathfrak{a}}_{\mathrm{q}}^{*}(P, 0)$ we have:

$$
\|\pi(\lambda) \varphi(\lambda)\| \leq C(1+|\lambda|)^{-n} e^{R|\operatorname{Re} \lambda|} .
$$

The supporting function of the closed ball $B$ in $\mathfrak{a}_{\mathrm{q}}$ of center 0 and radius $R \geq 0$ is given by $h_{B}(\lambda)=R|\operatorname{Re} \lambda|$. Using this in combination with Definition 8.9 we see that

$$
\mathcal{M}(G / H: \tau)=\bigcup_{S} \mathcal{M}(S, \pi)
$$

where $S$ ranges over the $W_{K \cap H}$-invariant compact subsets of $\mathfrak{a}_{\mathrm{q}}$. From Corollary $20.2(\mathrm{~b})$ we recall that the spaces in the right-hand side of (179) are Fréchet. Moreover, if $S_{1} \subset S_{2}$, then $\mathcal{M}\left(S_{1}, \pi\right) \subset \mathcal{M}\left(S_{2}, \pi\right)$, the inclusion map being continuous. Accordingly we equip $\mathcal{M}(G / H: \tau)$ with the direct limit locally convex topology.

In view of (179), the following result is an immediate consequence of Corollary 10.4 (and Remark 10.5).

Lemma 21.3 Let $D \in \mathbb{D}_{\pi}$. Then $D \mathcal{J}$ maps the pre Paley-Wiener space $\mathcal{M}(G / H: \tau)$ continuously into $C_{c}^{\infty}(G / H: \tau)$. 
Remark 21.4 It follows from Corollary 20.2 that there exists an $\epsilon>0$ such that $\pi \varphi$ is holomorphic on the open neighborhood $\mathfrak{a}_{\mathrm{q}}^{*}(P, \epsilon)$ of $\overline{\mathfrak{a}}_{\mathrm{q}}^{*}(P, 0)$, for every $\varphi \in \mathcal{M}(G / H: \tau)$. Moreover, the map $\varphi \mapsto \pi \varphi \mid \mathfrak{a}_{\mathrm{q}}^{*}(P, \epsilon)$ is continuous from $\mathcal{M}(G / H: \tau)$ to $\mathcal{O}\left(\mathfrak{a}_{\mathrm{q}}^{*}(P, \epsilon)\right) \otimes{ }^{\circ} \mathcal{C}(\tau)$. We may shrink $\epsilon$ to ensure that the polynomial $\pi$ has no zeros in $\mathfrak{a}_{\mathrm{q}}^{*}(\epsilon)$ (see $(64)$ and Lemma 8.1 (a)). Thus it also follows that every $\varphi \in \mathcal{M}(G / H: \tau)$ is holomorphic on the open neighborhood $\mathfrak{a}_{\mathrm{q}}^{*}(\epsilon)$ of $i \mathfrak{a}_{\mathrm{q}}^{*}$ and that the restriction map is continuous from $\mathcal{M}(G / H: \tau)$ to $\mathcal{O}\left(\mathfrak{a}_{\mathrm{q}}^{*}(\epsilon)\right) \otimes{ }^{\circ} \mathcal{C}(\tau)$.

From Theorem 8.11 and Theorem 15.1 we see that $\mathcal{F}$ maps $C_{S}^{\infty}(G / H: \tau)$ injectively and continuously into $\mathcal{M}(S, \pi)$, for any $W_{K \cap H^{-}}$invariant and compact subset $S$ of $\mathfrak{a}_{\mathrm{q}}$. Therefore $\mathcal{F}$ maps $C_{c}^{\infty}(G / H: \tau)$ injectively and continuously into $\mathcal{M}(G / H: \tau)$. If $f \in$ $C_{c}^{\infty}(G / H: \tau)$ then the coefficients in all Laurent series developments of $\mathcal{F} f$ satisfy all the linear relations coming from similar relations for Eisenstein integrals. In fact we have:

Lemma 21.5 Let $\partial_{1}, \ldots, \partial_{k} \in S\left(\mathfrak{a}_{\mathrm{q}}^{*}\right)$ be a finite collection of constant coefficient complex differential operators on $\mathfrak{a}_{\mathrm{q}}^{*}$, and let $\psi_{1}, \ldots, \psi_{k} \in{ }^{\circ} \mathcal{C}(\tau)$, and $\lambda_{1}, \ldots, \lambda_{k} \in \overline{\mathfrak{a}}_{\mathrm{q}}^{*}(P, 0)$. Then the relation

$$
\sum_{i=1}^{k} \partial_{i}\left[\pi(\lambda)\left\langle v \mid E^{\circ}\left(P: \psi_{i}:-\bar{\lambda}\right)\right\rangle\right]_{\lambda=\lambda_{i}}=0
$$

of elements in $C^{\infty}(G / H)$ holds for every $v \in V_{\tau}$ if and only if for all $f \in C_{c}^{\infty}(G / H: \tau)$ one has the relation:

$$
\sum_{i=1}^{k} \partial_{i}\left[\pi(\lambda)\left\langle\mathcal{F} f(\lambda) \mid \psi_{i}\right\rangle\right]_{\lambda=\lambda_{i}}=0 .
$$

Proof. For $v \in V_{\tau}$, let $E_{v}$ denote the left-hand side of (180), viewed as a function in $C^{\infty}(G / H)$. Define $E: G / H \rightarrow V_{\tau}^{*}$ by $E(x)(v)=E_{v}(x)$. Then $E \in C^{\infty}\left(G / H: \tau^{\vee}\right)$. The condition (180) holds for every $v \in V_{\tau}$ if and only if $E=0$, which in turn is equivalent to $\int_{G / H} E(x)(f(x)) d x=0$ for all $f \in C_{c}^{\infty}(G / H: \tau)$. By the definition of the Fourier transform the latter condition is equivalent to (181) for all $f \in C_{c}^{\infty}(G / H: \tau)$.

In the group case the extra relations (181) are known to determine $\mathcal{F}\left(C_{c}^{\infty}(G / H: \tau)\right)$ as a subspace of $\mathcal{M}(G / H: \tau)$ by the work of Campoli [16] in the split rank one case and Arthur [2] in the general case. This motivates the following definition.

Definition 21.6 The Paley-Wiener space $\mathrm{PW}(G / H: \tau)$ is defined to be the space of functions $\varphi \in \mathcal{M}(G / H: \tau)$ satisfying the following condition:

For all finite collections $\partial_{1}, \ldots, \partial_{k} \in S\left(\mathfrak{a}_{\mathrm{q}}^{*}\right), \psi_{1}, \ldots, \psi_{k} \in{ }^{\circ} \mathcal{C}(\tau)$, and $\lambda_{1}, \ldots, \lambda_{k} \in \overline{\mathfrak{a}}_{\mathrm{q}}^{*}(P, 0)$ for which one has the relation (180) of elements in $C^{\infty}(G / H)$ for every $v \in V_{\tau}$, one also has the relation:

$$
\sum_{i=1}^{k} \partial_{i}\left[\pi(\lambda)\left\langle\varphi(\lambda) \mid \psi_{i}\right\rangle\right]_{\lambda=\lambda_{i}}=0
$$


Let $\Omega$ be the open neighborhood of $\overline{\mathfrak{a}}_{\mathrm{q}}^{*}(P, 0)$ of Remark 21.4. If $\partial_{1}, \ldots, \partial_{k} \in S\left(\mathfrak{a}_{\mathrm{q}}^{*}\right)$, $\psi_{1}, \ldots, \psi_{k} \in{ }^{\circ} \mathcal{C}(\tau)$, and $\lambda_{1}, \ldots, \lambda_{k} \in \overline{\mathfrak{a}}_{\mathrm{q}}^{*}(P, 0)$ are as in the above definition, then the map

$$
\psi \mapsto \sum_{i=1}^{k} \partial_{i}\left[\pi(\lambda)\left\langle\varphi(\lambda) \mid \psi_{i}\right\rangle\right]_{\lambda=\lambda_{i}}
$$

is continuous from $\mathcal{O}(\Omega) \otimes{ }^{\circ} \mathcal{C}(\tau)$ to $\mathbb{C}$. In view of Remark 21.4 the map is also continuous from $\mathcal{M}(G / H: \tau)$ to $\mathbb{C}$. This implies that the space

$$
\mathrm{PW}_{S}(G / H: \tau):=\mathcal{M}(S, \pi) \cap \operatorname{PW}(G / H: \tau)
$$

is a closed subspace of the Fréchet space $\mathcal{M}(S, \pi)$; it thus becomes a Fréchet space in a natural way. From (179) we see that

$$
\operatorname{PW}(G / H: \tau)=\bigcup_{S} \operatorname{PW}_{S}(G / H: \tau),
$$

where $S$ ranges over the $W_{K \cap H}$-invariant compact subsets of $\mathfrak{a}_{\mathrm{q}}$. Moreover, if $S_{1} \subset S_{2}$, then $\mathrm{PW}_{S_{1}}(G / H: \tau) \subset \mathrm{PW}_{S_{2}}(G / H: \tau)$, the inclusion map being continuous. Accordingly we equip PW $(G / H: \tau)$ with the direct limit locally convex topology. Then the inclusion map $\mathrm{PW}(G / H: \tau) \subset \mathcal{M}(G / H: \tau)$ is continuous and has a closed image.

From the above discussion we now obtain:

Proposition 21.7 The Fourier transform $\mathcal{F}$ is an injective continuous linear map from $C_{c}^{\infty}(G / H: \tau)$ into $\mathrm{PW}(G / H: \tau)$. Moreover, if $S$ is a $W_{K \cap H}$-invariant compact subset of $\mathfrak{a}_{\mathrm{q}}$, then $\mathcal{F}$ maps $C_{S}^{\infty}(G / H: \tau)$ into $\mathrm{PW}_{S}(G / H: \tau)$.

Remark 21.8 We believe $\mathcal{F}$ is actually a linear isomorphism from $C_{c}^{\infty}(G / H: \tau)$ onto PW $(G / H: \tau)$. Although we do not know how to prove this in general, we will present a proof for the case $\operatorname{dim} \mathfrak{a}_{\mathrm{q}}=1$.

Before proceeding we note some consequences of the above conjecture. Suppose the conjecture is valid. Then if $S$ is a $W_{K \cap H}$-invariant compact convex subset of $\mathfrak{a}_{\mathrm{q}}$, the Fourier transform $\mathcal{F}$ restricts to an injective continuous linear map of Fréchet spaces $C_{S}^{\infty}(G / H: \tau) \rightarrow \mathrm{PW}_{S}(G / H: \tau)$. The restricted map is surjective as well. For let $\psi \in$ $\mathrm{PW}_{S}(G / H: \tau)$. Then by the conjecture there exists a function $f \in C_{c}^{\infty}(G / H: \tau)$ such that $\psi=\mathcal{F} f$. From Theorem 21.1 we deduce that $f \in C_{S}^{\infty}(G / H: \tau)$, whence the surjectivity. By the open mapping theorem for Fréchet spaces it then follows that $\mathcal{F}$ restricts to a topological linear isomorphism from $C_{S}^{\infty}(G / H: \tau)$ onto $\mathrm{PW}_{S}(G / H: \tau)$. Taking the limit over all $W_{K \cap H}$-invariant compact convex subsets of $\mathfrak{a}_{\mathrm{q}}$ we then obtain that $\mathcal{F}$ is actually a topological linear isomorphism from $C_{c}^{\infty}(G / H: \tau)$ onto $\operatorname{PW}(G / H: \tau)$.

The following result gives a useful reformulation of the relations in Definition 21.6 (compare with [2], p. 76). 
Lemma 21.9 Let $\varphi \in \mathcal{M}(G / H: \tau)$. Then $\varphi \in \operatorname{PW}(G / H: \tau)$ if and only if for all finite collections $\partial_{1}, \ldots, \partial_{k} \in S\left(\mathfrak{a}_{\mathrm{q}}^{*}\right)$ and $\lambda_{1}, \ldots, \lambda_{k} \in \overline{\mathfrak{a}}_{\mathrm{q}}^{*}(P, 0)$ there exists a function $f \in C_{c}^{\infty}(G / H: \tau)$ such that

$$
\partial_{i}[\pi(\lambda) \varphi(\lambda)]_{\lambda=\lambda_{i}}=\partial_{i}[\pi(\lambda) \mathcal{F} f(\lambda)]_{\lambda=\lambda_{i}} \quad(1 \leq i \leq k) .
$$

Proof. The 'if' part is obvious from Lemma 21.5. To see that the 'only if' part holds, let $\partial_{1}, \ldots, \partial_{k}$ and $\lambda_{1}, \ldots, \lambda_{k}$ be given. We equip the finite dimensional linear space $\left({ }^{\circ} \mathcal{C}(\tau)\right)^{k}$ with the product space Hilbert structure and define the linear map $L: \operatorname{PW}(G / H: \tau) \rightarrow$ $\left({ }^{\circ} \mathrm{C}(\tau)\right)^{k}$ by

$$
L(\phi)_{i}=\partial_{i}[\pi(\lambda) \phi(\lambda)]_{\lambda=\lambda_{i}} \quad(\phi \in \mathrm{PW}(G / H: \tau), i=1, \ldots, k) .
$$

Then we must show that $L(\phi) \in L\left(\mathcal{F} C_{c}^{\infty}(G / H: \tau)\right)$ for all $\phi \in \mathrm{PW}(G / H: \tau)$, or equivalently that the subspace $U:=L\left(\mathcal{F} C_{c}^{\infty}(G / H: \tau)\right)$ of $\operatorname{im} L$ in fact equals $\operatorname{im} L$. Since $\left({ }^{\circ} \mathrm{C}(\tau)\right)^{k}$ is finite dimensional this is equivalent to the assertion that for every $\psi=\left(\psi_{i}\right) \in$ $\left({ }^{\circ} \mathrm{C}(\tau)\right)^{k}$ we have that $\psi \perp U \Rightarrow \psi \perp \mathrm{im} L$. In view of Lemma 21.5 this is a consequence of Definition 21.6.

We will need the following result.

Lemma 21.10 Let $D \in \mathbb{D}_{\pi}$. Then for every $\varphi \in \mathcal{M}(G / H: \tau)$ we have

$$
\mathcal{F} D \mathcal{J} \varphi=\mu(D: \tau) \varphi
$$

Proof. By meromorphy of both members it suffices to establish this identity on $i \mathfrak{a}_{\mathrm{q}}^{*}$. Since det $\mu(D: \tau) \neq 0$, it suffices to establish the identity which arises from application of $\mu(D: \tau)$ to $(183)$; hence it suffices to prove (183) with $D^{2}$ instead of $D$. By the invariance (a) of Definition 21.2 it suffices to show that the resulting identity holds when tested against elements of $\left(\mathcal{S}\left(i \mathfrak{a}_{\mathrm{q}}^{*}\right) \otimes{ }^{\circ} \mathcal{C}(\tau)\right)^{W}$. Hence in view of Theorem 16.16 it suffices to show that

$$
\left\langle\mathcal{F} f \mid \mathcal{F} D^{2} \mathcal{J} \varphi\right\rangle=\left\langle\mathcal{F} f \mid \mu(D: \tau)^{2} \varphi\right\rangle
$$

for all $f \in \mathcal{C}(G / H: \tau)$. By continuity of the Fourier transform it suffices to establish the identity for $f$ in the dense subspace $C_{c}^{\infty}(G / H: \tau)$. The left-hand side of (184) then equals

$$
\begin{aligned}
\left\langle\mathcal{J} \mathcal{F} f \mid D^{2} \mathcal{J} \varphi\right\rangle & =\langle D \mathcal{J} \mathcal{F} f \mid D \mathcal{J} \varphi\rangle=\langle D f \mid D \mathcal{J} \varphi\rangle \\
& =\left\langle D^{2} f \mid \mathcal{J} \varphi\right\rangle=\left\langle\mathcal{F} D^{2} f \mid \varphi\right\rangle \\
& =\left\langle\mu(D: \tau)^{2} \mathcal{F} f \mid \varphi\right\rangle .
\end{aligned}
$$

The transpositions that have been carried out are all allowed, since they are of the form $\langle g \mid D h\rangle=\langle D g \mid h\rangle$, with $g, h \in C^{\infty}(G / H: \tau)$, and with either $g$ or $h$ compactly supported. The second equality follows from Theorem 14.1. Finally the last member of the equations (185) equals the right-hand side of (184) in view of (40) and since both $\mathcal{F} f$ and $\varphi$ are smooth functions on $i \mathfrak{a}_{\mathrm{q}}^{*}$, decreasing faster than $(1+|\lambda|)^{-n}$ for every $n$. 
Corollary 21.11 Let $D \in \mathbb{D}_{\pi}$. Then multiplication by $\mu(D: \tau)$ maps $\mathcal{M}(G / H: \tau)$ into the subspace $\mathcal{F}\left(C_{c}^{\infty}(G / H: \tau)\right)$ of $\operatorname{PW}(G / H: \tau)$.

Proof. This follows from combining Lemma 21.10 with Lemma 21.3 and Proposition 21.7.

In particular, if $G / H$ is a Riemannian symmetric space of the noncompact type we have seen in Remark 14.4 that $\pi=1$ and $1 \in D_{\pi}$. Hence it follows from the corollary above that $\mathcal{M}(G / H: \tau)=\mathcal{F}\left(C_{c}^{\infty}(G / H: \tau)\right)=\mathrm{PW}(G / H: \tau)$. Hence in this case (182) is fulfilled for all functions satisfying (a)-(c) in Definition 21.2. Moreover we have that the Fourier transform maps $C_{c}^{\infty}(G / H: \tau)$ onto the Paley-Wiener space. It can be shown that this assertion is equivalent with Helgason's Paley-Wiener theorem for the $\delta$-spherical Fourier transform, see [31], Ch. III, Thm. 5.11.

Notice that in general $\mathrm{PW}(G / H: \tau)$ is a proper subspace of $\mathcal{M}(G / H: \tau)$ (see for example [16], Thm. 3.4.2 and the succeeding example). However, in the split rank one case (i.e. $\operatorname{dim} \mathfrak{a}_{\mathrm{q}}=1$ ) the following corollary shows that only finitely many conditions of the form (182) are needed to characterize it as a subspace of $\mathcal{M}(G / H: \tau)$ (cf. [16], Observation 2.3.2, for the group case).

Corollary 21.12 Let $\operatorname{dim} \mathfrak{a}_{\mathrm{q}}=1$. Then the Paley-Wiener space PW $(G / H: \tau)$ has finite codimension in $\mathcal{M}(G / H: \tau)$.

Proof. Fix $D \in \mathbb{D}_{\pi}$. Then in view of Corollary 21.11 it suffices to show that the space $\mu(D: \tau) \mathcal{M}(G / H: \tau)$ has finite codimension in $\mathcal{M}(G / H: \tau)$. Let $\left\{\lambda_{1}, \ldots, \lambda_{k}\right\}$ be the set of zeros of $\operatorname{det} \mu(D: \tau)$ in $\overline{\mathfrak{a}}_{\mathrm{q}}^{*}(P, 0)$. For $1 \leq j \leq k$, let $m_{j}$ be the order of the zero of det $\mu(D: \tau)$ at $\lambda_{j}$. Let $V$ be the subspace of $\mathcal{M}(G / H: \tau)$ consisting of the functions $\varphi$ for which $\pi \varphi$ vanishes up to the order $m_{j}$ at every $\lambda_{j}(1 \leq j \leq k)$. Then $V$ has finite codimension. Moreover, if $\varphi \in V$, then $\pi \mu(D: \tau)^{-1} \varphi$ is holomorphic on $\overline{\mathfrak{a}}_{\mathrm{q}}^{*}(P, 0)$. Using (38) with $Q=P$ one now readily deduces that $\mu(D: \tau)^{-1} \varphi \in \mathcal{M}(G / H: \tau)$. Hence $V$ is contained in $\mu(D: \tau) \mathcal{M}(G / H: \tau)$, and therefore the latter space has finite codimension.

Lemma 21.13 Assume that $\operatorname{dim} \mathfrak{a}_{\mathrm{q}}=1$, and let $D \in \mathbb{D}_{\pi}$. Then for every function $\varphi \in$ $\mathrm{PW}(G / H: \tau)$ there exists a function $f \in C_{c}^{\infty}(G / H: \tau)$ such that $\varphi-\mathcal{F} f$ belongs to $\mu(D: \tau) \mathcal{M}(G / H: \tau)$.

Proof. Let $\varphi \in \mathrm{PW}(G / H: \tau)$, and let the subspace $V$ of $\mathcal{M}(G / H: \tau)$ be defined as in the proof of Corollary 21.12. Then by Lemma 21.9 there exists a $f \in C_{c}^{\infty}(G / H: \tau)$ such that $\varphi-\mathcal{F} f \in V$. In the proof of Corollary 21.12 we saw that $V \subset \mu(D: \tau) \mathcal{M}(G / H: \tau)$.

We can now prove a full Paley-Wiener theorem for the case that $G / H$ has split rank one. 
Theorem 21.14 Assume $\operatorname{dim} \mathfrak{a}_{\mathrm{q}}=1$. Then $\mathcal{F}$ is a topological linear isomorphism from $C_{c}^{\infty}(G / H: \tau)$ onto $\operatorname{PW}(G / H: \tau)$.

Proof. By Remark 21.8 it suffices to prove bijectivity, and by Proposition 21.7 it remains to prove surjectivity. Let $\varphi \in \operatorname{PW}(G / H: \tau)$ be given. Fix $D \in \mathbb{D}_{\pi}$, and let $f \in C_{c}^{\infty}(G / H: \tau)$ be as in Lemma 21.13. Then $\varphi-\mathcal{F} f$ belongs to $\mu(D: \tau) \mathcal{M}(G / H: \tau)$, hence to $\mathcal{F}\left(C_{c}^{\infty}(G / H: \tau)\right)$, by Corollary 21.11. Hence also $\varphi$ belongs to the latter space.

\section{References}

[1] J.-Ph. Anker, The spherical Fourier transform of rapidly decreasing functions - a simple proof of a characterization due to Harish-Chandra, Helgason, Trombi and Varadarajan. J. Funct. Anal. 96 (1991), 331-349.

[2] J. Arthur, A Paley-Wiener theorem for real reductive groups. Acta Math. 150 (1983), 1-89.

[3] E.P. van den Ban, A convexity theorem for semisimple symmetric spaces. Pac. J. Math. 124 (1986), 21-55.

[4] E.P. van den Ban, Asymptotic behaviour of matrix coefficients related to reductive symmetric spaces. Proc. Kon. Nederl. Akad. Wet. 90 (1987), 225-249.

[5] E.P. van den Ban, Invariant differential operators on a semisimple symmetric space and finite multiplicities in a Plancherel formula. Arkiv för mat. 25 (1987), 175-187.

[6] E.P. van den Ban, The principal series for a reductive symmetric space I. H-fixed distribution vectors. Ann. sci. Ec. Norm. Sup. 21 (1988), 359-412.

[7] E.P. van den Ban, The principal series for a reductive symmetric space II. Eisenstein integrals. J. Funct. Anal. 109 (1992), 331-441.

[8] E.P. van den Ban, The action of intertwining operators on spherical vectors in the minimal principal series of a reductive symmetric space. In preparation.

[9] E.P. van den Ban, J. Carmona and P. Delorme, Paquets d'ondes dans l'espace de Schwartz d'un espace symétrique réductif. To appear in J. Funct. Anal.

[10] E.P. van den Ban, M. Flensted-Jensen and H. Schlichtkrull, Basic harmonic analysis for pseudo-Riemannian symmetric spaces. pp. 69 - 101 in: Noncompact Lie Groups and Some of their Applications, 69-101, E.A. Tanner \& R. Wilson (eds.) (proc. of the San Antonio NATO workshop, 1993), Kluwer Academic Publishers, 1994.

[11] E.P. van den Ban and H. Schlichtkrull, Multiplicities in the Plancherel decomposition for a semisimple symmetric space. Contemporary Math. 145 (1993), 163-180. 
[12] E.P. van den Ban and H. Schlichtkrull, Convexity for invariant differential operators on a semisimple symmetric space. Compos. Math. 89 (1993), 301-313.

[13] E.P. van den Ban and H. Schlichtkrull, Fourier transforms on a semisimple symmetric space. Preprint no. 888, Universiteit Utrecht, November 1994.

[14] E.P. van den Ban and H. Schlichtkrull, Expansions for Eisenstein integrals on a semisimple symmetric space. In preparation.

[15] N. Bopp and P. Harinck, Formule de Plancherel pour $G L(n, R) / U(p, q)$. J. reine und angew. Math. 428 (1992), 45-95.

[16] O. A. Campoli, Paley-Wiener type theorems for rank-1 semisimple Lie groups. Rev. Union Mat. Argent. 29 (1980), 197-221.

[17] W. Casselman and D. Miliçić, Asymptotic behaviour of matrix coefficients of admissible representations. Duke Math. J. 49 (1982), 869-930.

[18] G. van Dijk and M. Poel, The Plancherel formula for the pseudo-Riemannian space $G L(n, R) / G L(n-1, R)$. Compos. Math. 58 (1986), 371-397.

[19] J. Faraut, Distributions sphériques sur les espaces hyperboliques. J. Math. Pure Appl. 58 (1979), 369-444.

[20] M. Flensted-Jensen, Discrete series for semisimple symmetric spaces. Ann. of Math. 111 (1980), 253-311.

[21] R. Gangolli, On the Plancherel formula and the Paley-Wiener theorem for spherical functions on semisimple Lie groups. Ann. of Math. 93 (1971), 150-165.

[22] Harish-Chandra, Spherical functions on a semisimple Lie group, I. Amer. J. Math. 80 (19.58), 241-310.

[23] Harish-Chandra, Spherical functions on a semisimple Lie group, II. Amer. J. Math. 80 (1958), 553-613.

[24] Harish-Chandra, Discrete series for semisimple Lie groups, II. Explicit determination of the characters. Acta Math. 116 (1966), 1-111.

[25] Harish-Chandra, Harmonic analysis on real reductive groups I. The theory of the constant term. J. Funct. Anal. 19 (1975), 104-204.

[26] Harish-Chandra, Harmonic analysis on real reductive groups II. Wave packets in the Schwartz space. Invent. Math. 36 (1976), 1-55.

[27] Harish-Chandra, Harmonic analysis on real reductive groups III. The Maass-Selberg relations and the Plancherel formula. Ann. Math. 104 (1976), 117-201. 
[28] G. Heckman and H. Schlichtkrull, Harmonic Analysis and Special Functions on Symmetric Spaces. Academic Press, San Diego 1994.

[29] S. Helgason, A duality for symmetric spaces with applications to group representations. Adv. in Math. 5 (1970), 1-154.

[30] S. Helgason, Groups and Geometric Analysis. Academic Press, Orlando 1984.

[31] S. Helgason, Geometric Analysis on Symmetric Spaces. Amer. Math. Soc., Providence 1994.

[32] L. Hörmander, The Analysis of Linear Partial Differential Operators I. SpringerVerlag, Berlin-Heidelberg-New York-Tokyo, 1983.

[33] A.W. Knapp and E.M. Stein, Intertwining operators for semisimple groups, II. Invent. Math. 60 (1980), 9-84.

[34] V.F. Molchanov, Plancherel's formula for pseudo-Riemannian symmetric spaces of rank 1. Sov. Math. Dokl. 34 (1987), 323-326.

[35] T. Oshima and T. Matsuki, A description of discrete series for semisimple symmetric spaces. Adv. Studies in Pure Math. 4 (1984), 331-390.

[36] J. Rosenberg, A quick proof of Harish-Chandra's Plancherel theorem for spherical functions on a semisimple Lie group. Proc. Amer. Math. Soc. 63 (1977), 143-149.

[37] H. Schlichtkrull, Hyperfunctions and harmonic analysis on symmetric spaces. Birkhäuser, Boston 1984.

[38] N.R. Wallach, Real Reductive Groups I. Academic Press, Inc., Boston 1988.

E.P. van den Ban

Mathematisch Instituut

Universiteit Utrecht

PO Box 80010

3508 TA Utrecht

Netherlands

Email: ban@math.ruu.nl
H. Schlichtkrull

Department of Mathematics and Physics

The Royal Veterinary and Agricultural University

Thorvaldsensvej 40

1871 Frederiksberg C

Denmark

Email: hs@dina.kvl.dk 